Copyright

by

Olabode Ijasan

2013 
The Dissertation Committee for Olabode Ijasan Certifies that this is the approved version of the following dissertation:

\section{Inversion-Based Petrophysical Interpretation of Logging-While-Drilling Nuclear and Resistivity Measurements}

Committee:

Carlos Torres-Verdín, Supervisor

Steven L. Bryant

William E. Preeg

Erich A. Schneider

Kamy Sepehrnoori 


\title{
Inversion-Based Petrophysical Interpretation of Logging-While-Drilling Nuclear and Resistivity Measurements
}

by

Olabode Ijasan, B.S.; M.S.E.

\author{
Dissertation \\ Presented to the Faculty of the Graduate School of \\ The University of Texas at Austin \\ in Partial Fulfillment \\ of the Requirements \\ for the Degree of \\ Doctor of Philosophy
}

The University of Texas at Austin

August, 2013 


\section{Dedication}

To my family, for their unconditional support and excruciating tolerance. 


\section{Acknowledgements}

I would like to express my sincere gratitude to my advisor, Dr. Carlos TorresVerdín for his consistent support and motivation. I am grateful to him for shaping my thought processes during my graduate studies. Special thanks to my co-supervisor, Dr. Bill Preeg for his priceless contributions to the developments in this dissertation. The technical advances in this dissertation would not have been possible without him. To Dr. Erich Schneider, I say thank you for the technical discussions and wonderful insights. I would like to acknowledge the members of my dissertation committee, Dr. Steven Bryant and Dr. Kamy Sepehrnoori, for their time, support, and reviews of this dissertation.

The work reported in this dissertation was funded by The University of Texas at Austin's Research Consortium on Formation Evaluation, jointly sponsored by Afren, Anadarko, Apache, Aramco, Baker-Hughes, BG, BHP Billiton, BP, Chevron, ConocoPhillips, COSL, ENI, ExxonMobil, Halliburton, Hess, Maersk, Marathon Oil Corporation, Mexican Institute for Petroleum, Nexen, ONGC, OXY, Petrobras, PTT Exploration and Production, Repsol, RWE, Schlumberger, Shell, Statoil, Total, Weatherford, Wintershall, and Woodside Petroleum Limited.

Special thanks to Rey Casanova for his administrative support at the formation evaluation research consortium. I'm grateful to Cheryl Kruzie and Frankie Hart for their help and support in the petroleum engineering department. To Roger Terzian and Tim Guinn, thank you for all your support and efforts toward the management of my desktop and cluster computers. I acknowledge the Texas Advanced Computing Center (TACC) at The University of Texas at Austin for providing high-performance computing resources

for performing the Monte Carlo simulations reported in this dissertation. I'm appreciative 
of the petroleum engineering department and college of engineering for the travel and financial grants to attend professional and industry conferences.

I was fortunate to spend two summers as an intern at the Schlumberger-Doll Research Center in Cambridge, Massachusetts. I am immensely grateful to Schlumberger for the internship opportunities and technical contributions toward the developments documented in this dissertation. Special thanks to Darwin Ellis, Joe Chiaramonte, Charlie Case, and Brad Roscoe for their support during my internships. Their expert contributions toward some of the advances in this research have added tremendous value to this dissertation. I would like to express my gratitude to John Rasmus, Jeff Miles, and Dzevat Omeragic for their technical reviews and expert opinions. Additionally, special thanks to Duncan Mardon, Russell Spears, and Dale Fitz at ExxonMobil. My prior internship experiences at ExxonMobil were invaluable toward the advancement of my career. I am especially grateful to ExxonMobil for sponsorship to the Society of Exploration Geophysicists 2012 Student Education Program.

Many thanks to Alberto Mendoza and Zoya Heidari for paving the way(s) for the research documented in this dissertation. I would like to thank my friends, colleagues, and office mates: Edwin Ortega, Tatyana Torskaya, Chicheng Xu, Oyinkan Ajayi, Hamid Beik, Essi Kwabi, Vahid Shabro, Amir Frooqnia, Abhishek Bansal, Haryanto Adiguna, Hyungjoo Lee, Dipo Olukitibi, Rebecca Gao, Shan Huang, Gbenga Alabi, Prince Azom, Andy Popielski, Elton Ferreria, Siddharth Mishra, Philippe Marouby, Kanay Jerath, Jordan Mimoun, Ankur Ghandi, and Shaina Kelly. Additional thanks to Ben Voss and Paul Linden for their help with some aspects of the algorithms in this research.

My sincere gratitude to the Ojifinnis and Olapades for their selfless hospitality during my internships. Special thanks to a friend, Chimaobi Onwuchekwa for his philosophical motivation and inspiration toward a spiritual development. 
Finally, I'm immensely grateful to my family for their support and patience throughout this journey. To Oseola, Olamide, Oluwakemi, Olaolopin, and Adelehin, thank you so much for all your perseverance, prayers, and yearn for my success. 


\title{
Inversion-Based Petrophysical Interpretation of Logging-While-Drilling Nuclear and Resistivity Measurements
}

\author{
Olabode Ijasan, Ph.D. \\ The University of Texas at Austin, 2013
}

Supervisor: Carlos Torres-Verdín

Undulating well trajectories are often drilled to improve length exposure to rock formations, target desirable hydrocarbon-saturated zones, and enhance resolution of borehole measurements. Despite these merits, undulating wells can introduce adverse conditions to the interpretation of borehole measurements which are seldom observed in vertical wells penetrating horizontal layers. Common examples are polarization horns observed across formation bed boundaries in borehole resistivity measurements acquired in highly-deviated wells. Consequently, conventional interpretation practices developed for vertical wells can yield inaccurate results in $\mathrm{HA} / \mathrm{HZ}$ wells. A reliable approach to account for well trajectory and bed-boundary effects in the petrophysical interpretation of well logs is the application of forward and inverse modeling techniques because of their explicit use of measurement response functions.

The main objective of this dissertation is to develop inversion-based petrophysical interpretation methods that quantitatively integrate logging-while-drilling (LWD) multisector nuclear (i.e., density, neutron porosity, photoelectric factor, natural gamma ray) and multi-array propagation resistivity measurements. Under the assumption of a multilayer formation model, the inversion approach estimates formation properties specific to a given measurement domain by numerically reproducing the available measurements. viii 
Subsequently, compositional multi-mineral analysis of inverted layer-by-layer properties is implemented for volumetric estimation of rock and fluid constituents.

The most important prerequisite for efficient petrophysical inversion is fast and accurate forward models that incorporate specific measurement response functions for numerical simulation of LWD measurements. In the nuclear measurement domain, firstorder perturbation theory and flux sensitivity functions (FSFs) are reliable and accurate for rapid numerical simulation. Albeit efficient, these first-order approximations can be inaccurate when modeling neutron porosity logs, especially in the presence of borehole environmental effects (tool standoff or/and invasion) and across highly contrasting beds and complex formation geometries. Accordingly, a secondary thrust of this dissertation is the introduction of two new methods for improving the accuracy of rapid numerical simulation of LWD neutron porosity measurements. The two methods include: (1) a neutron-density petrophysical parameterization approach for describing formation macroscopic cross section, and (2) a one-group neutron diffusion flux-difference method for estimating perturbed spatial neutron porosity fluxes. Both methods are validated with full Monte Carlo (MC) calculations of spatial neutron detector FSFs and subsequent simulations of neutron porosity $\operatorname{logs}$ in the presence of LWD azimuthal standoff, invasion, and highly dipping beds.

Analysis of field and synthetic verification examples with the combined resistivity-nuclear inversion method confirms that inversion-based estimation of hydrocarbon pore volume in $\mathrm{HA} / \mathrm{HZ}$ wells is more accurate than conventional well-log analysis. Estimated hydrocarbon pore volume from conventional analysis can give rise to errors as high as $15 \%$ in undulating $\mathrm{HA} / \mathrm{HZ}$ intervals. 


\section{Table of Contents}

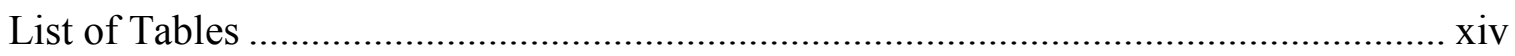

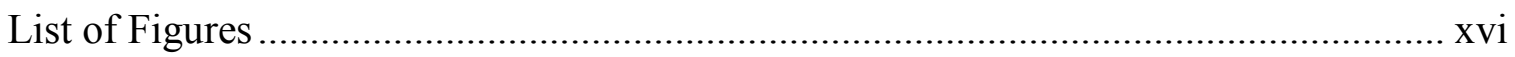

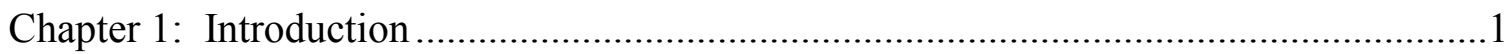

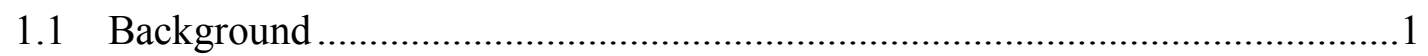

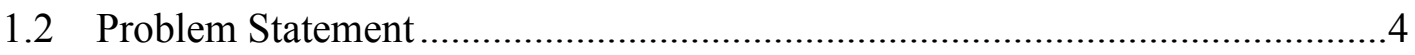

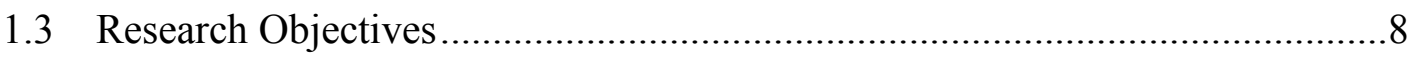

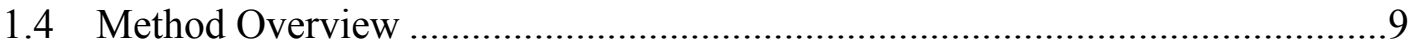

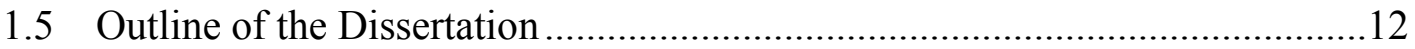

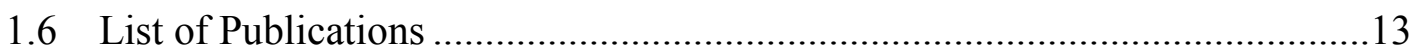

1.6.1 Refereed Journal Publications.......................................................13

1.6.2 Refereed Conference Proceedings ………………………………....14

Chapter 2: Fast-Forward Modeling of Logging-While-Drilling Neutron Porosity

Measurements in the Presence of Borehole and Environmental Effects ...................16

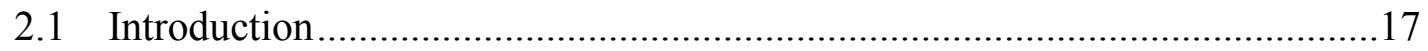

$2.2 F_{p}$ Formulation and Parameter Estimation.................................................19

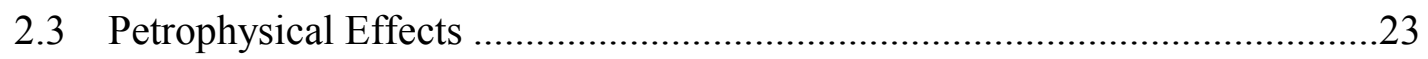

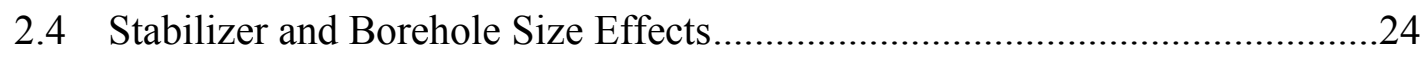

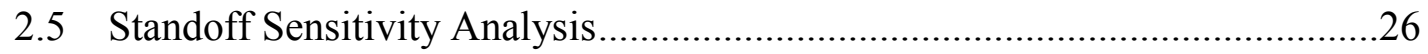

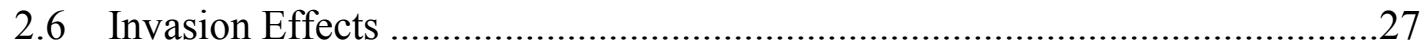

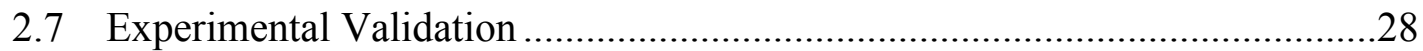

2.8 Modeling LWD Neutron Porosity Measurements in High-Angle/Horizontal

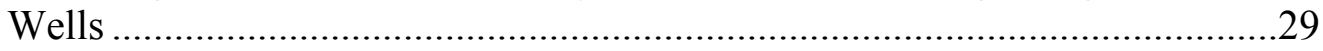

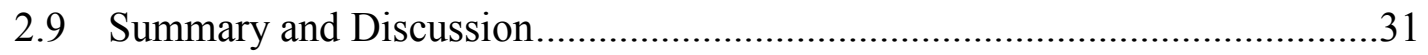

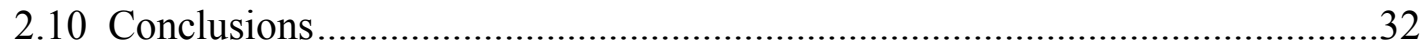

Chapter 3: Fast Modeling of Borehole Neutron Porosity Measurements with a New Spatial Transport-Diffusion Approximation.............................................................47

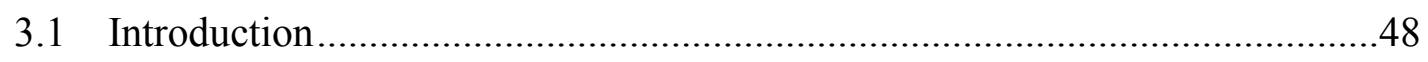

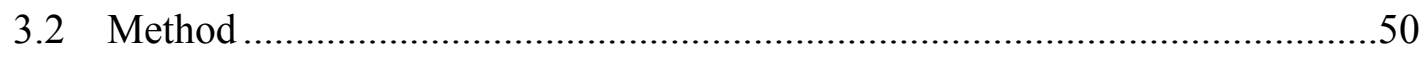


3.2.1 Diffusion Formulation ………………...................................

3.2.2 Boundary Conditions and Flux Continuity ……………………......54

3.2.3 Transport Correction ..................................................................54

3.2.4 Detector Response Perturbation....................................................55

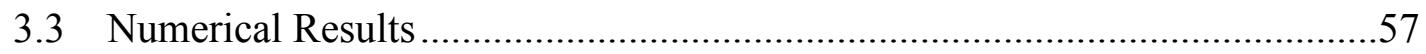

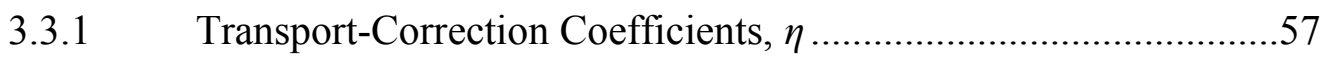

3.3.2 Diffusion Distributed Functions in Homogeneous Formations .....58

3.4 Benchmark Results: Borehole and Environmental Conditions ........................60

3.4.1 Borehole Standoff Effects in LWD Measurements ........................60

3.4.2 Invasion Effects in Wireline Measurements ....................................62

3.5 Modeling Applications in High-Angle and Horizontal Wells .........................63

3.5.1 Synthetic Case of a High-Angle Well in a Slick Borehole .............63

3.5.2 Synthetic Case of a High-Angle Well with Invasion Effects ........65

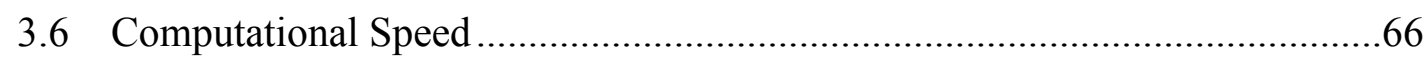

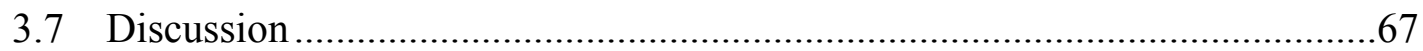

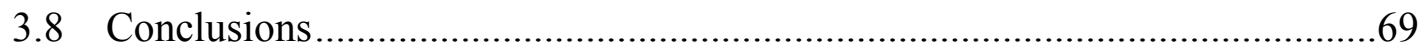

Chapter 4: Inversion-Based Petrophysical Interpretation of Logging-While-Drilling Nuclear and Resistivity Measurements....................................................................92

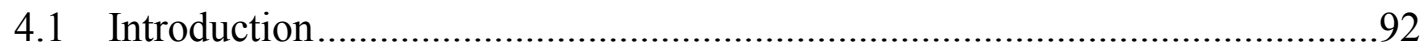

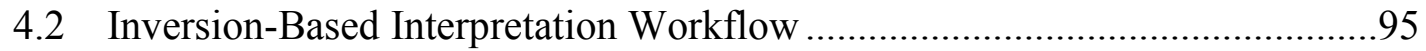

4.2.1 Consolidated Gamma Geometrical Model.....................................95

4.2.2 Measurement Domains: Property Parameterization and Data Space

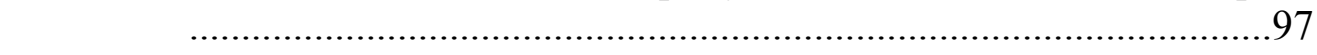

4.2.3 Overview of Inversion Method ....................................................

4.2.4 Compositional Petrophysics.........................................................100

4.3 Application to High-Angle and Horizontal Wells ………………...................100

4.3.1 Synthetic Benchmark Example.....................................................101

4.3.2 Field Case Example in Highly-Deviated Well...............................103

4.3.3 Field Case Example in Horizontal Well .......................................105

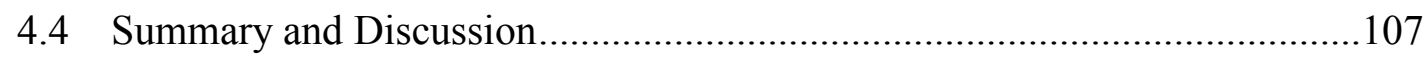

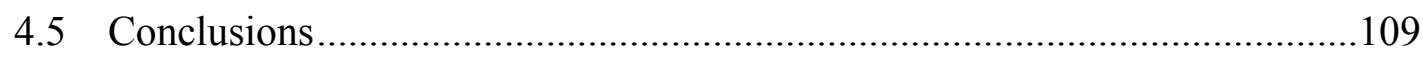


Chapter 5: Interpretation of Porosity and Fluid Constituents from Well Logs Using an Interactive Neutron-Density Matrix Scale ........................................................126

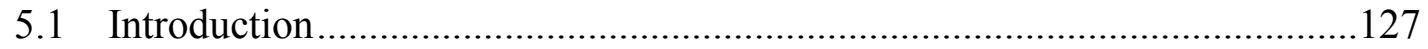

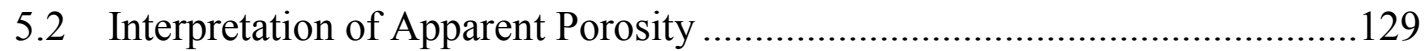

5.2.1 Density Apparent Porosity ………………….............................129

5.2.2 Neutron Apparent Porosity ……………….................................130

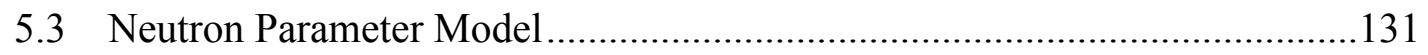

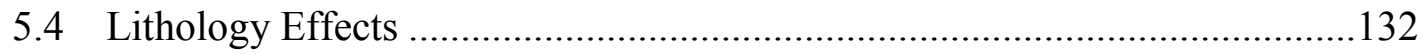

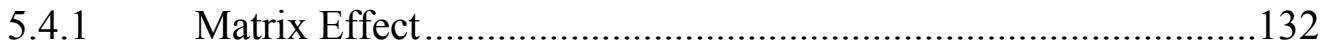

5.4.2 Shale-Hydroxyl or Matrix-Hydrogen Effect...............................133

5.5 Fluid and Hydrocarbon Saturation Effects ..................................................134

5.6 Interactive Analysis of Matrix and Fluid Effects...........................................135

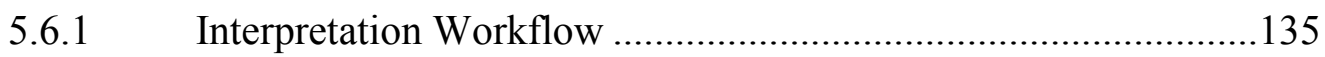

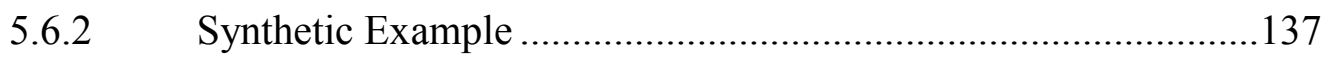

5.7 Field Examples of Application …………………….................................138

5.7.1 Field Example I, Gas-Bearing Carbonate ....................................139

5.7.2 Field Example II, Oil-Bearing Shale Example ..............................140

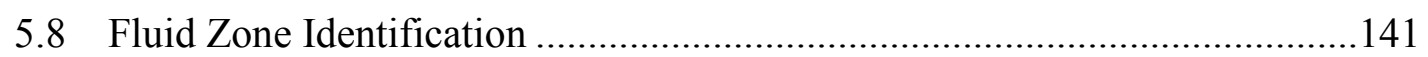

5.8.1 Field Example III, Identification of Hydrocarbon Fluid Contacts in a North Sea Siliciclastic Reservoir 141

5.8.2 Field Example IV, Identification of Reservoir Compartments in the Deepwater Gulf of Mexico ...............................................................142

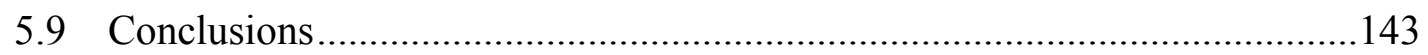

Chapter 6: Summary, Conclusions, and Recommendations .............................................156

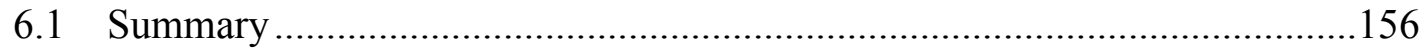

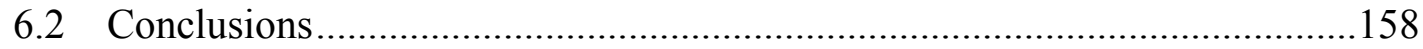

6.2.1 General Conclusions about the Significance to Formation Evaluation and Best Practices for Petrophysical Modeling and Inversion

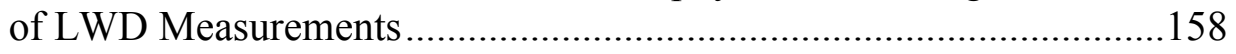

6.2.2 Part One: Fast-Forward Modeling of Logging-While-Drilling Neutron Porosity Measurements in the Presence of Borehole and Environmental Effects 162

$$
\text { xii }
$$


6.2.3 Part One: Fast Modeling of Borehole Neutron Porosity Measurements with a New Spatial Transport-Diffusion Approximation .163

6.2.4 Part Two: Inversion-Based Petrophysical Interpretation of Logging-While-Drilling Nuclear and Resistivity Measurements ..........165

6.2.5 Part Three: Interpretation of Porosity and Fluid Constituents from Well Logs Using an Interactive Neutron-Density Matrix Scale............168

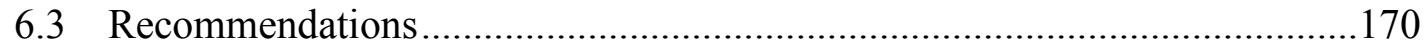

Appendix A: Synthetic Examples of Flux-Difference Modeling of Neutron Porosity

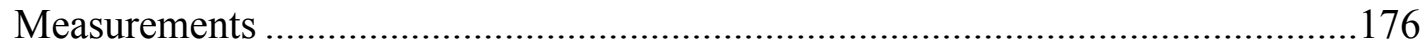

A.1 Synthetic Case of a High-Angle Well with Standoff Effects ................176

A.2 Borehole Standoff Effects in Wireline Measurements ...........................176

A.3 Synthetic Case of a High-Angle Well with Thin Bed Effects ...............178

Appendix B: Computational Tools for Inversion of Nuclear and Resistivity

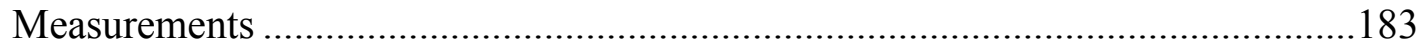

B.1 Nonlinear Separate Inversion in LWD Measurement Domains ...........183

B.1.1 Data and Parameter Space ......................................................183

B.1.2 Numerical Construction of Operator Matrices ……………......184

B.1.3 Finite-Difference Approximation of Operator Matrices ..........185

B.2 SNUPAR-Based Compositional Solver..................................................186

Appendix C: Effects of Formation Anisotropy and Polarization Horns on Layer-Based Inversion of Propagation Resistivity Measurements .............................................189

C.1 Synthetic Example ..............................................................................189

C.2 Field Case Example ........................................................................191

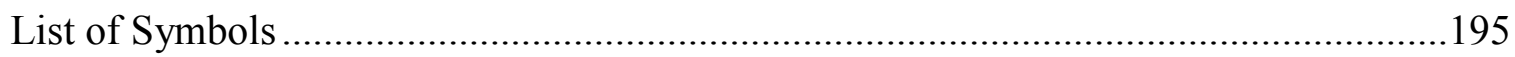

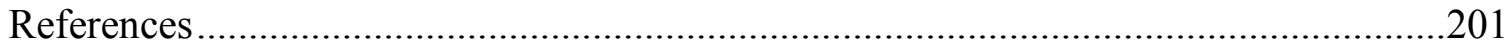

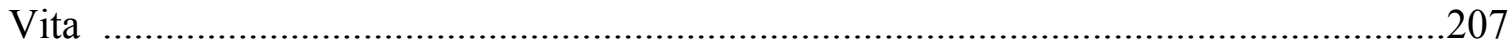




\section{List of Tables}

Table 2.1: $\quad F_{p}$ formulation coefficients, for limestone porosity units.

Table 3.1: Transport-correction coefficients, $\eta$, calculated for LWD and wireline tools, per detector.

Table 3.2: Summary of homogeneous formation base-cases and their neutron porosity $\log$ responses in limestone porosity units (pu). . .72

Table 3.3: Summary of assumed properties for the synthetic layered earth model. ......73

Table 3.4: Summary of assumed properties for the approximate "teardrop" invasion profile in the synthetic earth model.

Table 3.5: Summarized CPU times, quantified in the numerical simulation of neutron porosity measurements, of benchmark and LWD HA/HZ examples using MCNP, LIR, and DFD methods. .75

Table 4.1: Depth-periodic layer properties, compositions, and thicknesses assumed in the synthetic example. 110

Table 4.2: Petrophysical properties assumed in the synthetic example. 111

Table 4.3: Multi-mineral and fluid component models assumed in the West Africa hydrocarbon field examples.

Table 5.1: Layer properties assumed in the Synthetic Example.

Table 5.2: Summary of assumed Archie's parameters and fluid properties for the Synthetic Example. 145

Table 5.3: Summary of assumed fluid properties and Archie's parameters for Field Example I, gas-bearing carbonate. 146

Table 5.4: Summary of assumed fluid properties and Archie's parameters for Field Example II, oil-bearing shale. 146 
Table 5.5: Summary of assumed properties and Archie's parameters for Field Example III, North Sea siliciclastic reservoir. . 147

Table 5.6: Summary of assumed properties and Archie's parameters for Field Example IV, deepwater Gulf of Mexico reservoir.

Table C.1: Electrical anisotropy properties assumed in the synthetic example.............192 


\section{List of Figures}

Figure 1.1: Field example of undulating well trajectory effects in LWD compensated density measurements. (a) Compensated density image showing high and low density layers, and the characteristic "bull's-eye" feature in undulating HA/HZ wells. (b) Description of well trajectory and formation geometrical properties.......

Figure 2.1: Near and far detector counts, in various porosities of limestone freshwater-, gas-, and saltwater-saturated formations, versus inverse of formation migration length.

Figure 2.2: (a) Regression of far and near detector counts, across base cases of various fluid properties, with $F_{p}$ parameterization. (b) Computed BPHI (neutron porosity) differences, due to residuals in panel a, versus the average of near and far detector $F_{p}$

Figure 2.3: Benchmark results in slick 8-in. borehole. (a) Detector counts obtained from MCNP and FSF- $F_{p}$ FFM for freshwater- and gas-saturated limestone formations versus true pore volume in \%. (b) Density (DPHI) and neutron (BPHI) porosity logs versus true pore volume. Circular and square points represent water- and gas-saturated formations, respectively. (c) Calculated porosity differences between $\mathrm{MC}$ and $\mathrm{FSF}-F_{p}$ methods. .36 
Figure 2.4: Benchmark results in slick 8-in. borehole. (a) Detector counts obtained from MCNP and FSF- $F_{p}$ FFM for freshwater- and gas-saturated sandstone formations versus true pore volume in \%. (b) Density (DPHI) and neutron (BPHI) porosity logs versus true pore volume. Circular and square points represent water- and gas-saturated formations, respectively. (c) Calculated porosity differences between $\mathrm{MC}$ and $\mathrm{FSF}-F_{p}$ methods.

Figure 2.5: Benchmark results in slick 8-in. borehole. (a) Detector counts obtained from MCNP and FSF- $F_{p}$ FFM for freshwater- and gas-saturated dolomite formations versus true pore volume in \%. (b) Density (DPHI) and neutron (BPHI) porosity logs versus true pore volume. Circular and square points represent water- and gas-saturated formations, respectively. (c) Calculated porosity differences between MC and FSF- $F_{p}$ methods................................38

Figure 2.6: Schematic diagram showing LWD tool-borehole geometry. (a) Vertical cross-section showing tool housing, stabilizer, and borehole. Horizontal cross-section showing tool at (b) bottom, (c) left, (d) top orientation in the hole 39

Figure 2.7: Benchmark results of an 8.25-in. stabilizer at the bottom of a 10-in. borehole. (a) Detector counts obtained from MCNP and FSF- $F_{p}$ FFM for freshwater- and gas-saturated limestone formations versus true pore volume in \%. (b) Density (DPHI) and neutron (BPHI) porosity logs versus true pore volume. Circular and square points represent water- and gas- saturated formations, respectively. (c) Calculated porosity differences between $\mathrm{MC}$ and FSF- $F_{p}$ methods. .40 
Figure 2.8: Benchmark results of an 8.25-in. stabilizer at the top of a 10-in. borehole. (a) Detector counts obtained from MCNP and FSF- $F_{p}$ FFM for freshwater- and gas-saturated limestone formations versus true pore volume in \%. (b) Density (DPHI) and neutron (BPHI) porosity logs versus true pore volume. Circular and square points represent water- and gas- saturated formations, respectively. (c) Calculated porosity differences between MC and FSF- $F_{p}$ methods.

Figure 2.9: LWD standoff sensitivity analysis of FSF- $F_{p}$ FFM, in comparison to MCNP, around the borehole using 7.875 in., 8.25 in., and 9.375 in. stabilizer sizes. Calculated porosity differences in (a) 8-in., 8.5-in., and 9.5-in. borehole sizes, (b) 10-in. borehole size, and (c) 12-in. borehole size

Figure 2.10: Detector counts for $\mathrm{MC}$ and $\mathrm{FSF}-F_{p}$ methods versus radial distance of invasion front from the borehole wall for (a) far detector with 7.12 in. DOI, and (b) near detector with 4.26 in. DOI. (c) DPHI and BPHI porosity logs versus radial distance of invasion front. $\mathrm{MC}$ and $\mathrm{FSF}-F_{p}$ BPHIs in black and green curves, respectively. Red vertical line represents 3.88 in. density DOI, while green vertical line represents 9 in. neutron porosity DOI. (d) BPHI differences between MC and FSF- $F_{p}$ methods.

Figure 2.11: Experimental validation of FSF- $F_{p}$ FFM, with LIR, in a 10-inch borehole. (a) Far and near detector counts per second. (b) Near-to-far count ratios. (c) BPHI porosity logs . .44 
Figure 2.12: Comparison of LWD MCNP and FSF- $F_{p}$ simulation results in an $89^{\circ}$ well across a synthetic earth model of 2.5 -ft layer thicknesses. Logarithm of far detector counts obtained with (a) MCNP and (b) FSF- $F_{p}$ methods, versus true vertical depth. Logarithm of near detector counts obtained with (c) MCNP and (d) FSF- $F_{p}$ methods. BPHI neutron porosity image from (e) MCNP and (f) FSF- $F_{p}$ methods. (g) Simulated compensated density image. (h) Bottom quadrant DPHI and BPHI porosity logs. (i) BPHI sector differences between FSF and MCNP methods. .45

Figure 2.13: Comparison of LWD MCNP and FSF- $F_{p}$ simulation results in an $85^{\circ}$ well across a synthetic earth model of 0.5 -ft layer thickness. Logarithm of far detector counts obtained with (a) MCNP and (b) FSF- $F_{p}$ methods, versus true vertical depth. Logarithm of near detector counts obtained with (c) MCNP and (d) FSF- $F_{p}$ methods. BPHI neutron porosity image from (e) MCNP and (f) FSF- $F_{p}$ methods. (g) Simulated compensated density image. (h) Bottom quadrant DPHI and BPHI porosity logs. (i) BPHI sector differences between FSF and MCNP methods. .46

Figure 3.1: Normalized 2D diffusion kernels of neutron point source located at $\mathbf{r}_{S}=\mathbf{r}_{S}\left(r_{S}, z_{S}\right)=\left(0.25 r_{e x}, 0.5 H_{e x}\right)$ for $\alpha_{B}^{-1}=30 \mathrm{~cm}$, using equations 3.3 and 3.4 .

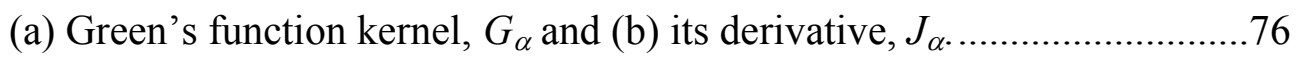

Figure 3.2: Flow diagram of the flux-fitting method used to calculate transportcorrection coefficient, $\eta$. .77 
Figure 3.3: Calculation of the transport-correction coefficient, $\eta$ across various basecases of water- and gas-filled lithologies. (a) Cross-plot of modified travel length, $L_{I E}^{\prime}$ and diffusion length, $L_{d}$. (b) Cross-plot of modified travel length, $L_{I E}^{\prime}$ and migration length, $L_{m}$ for an LWD neutron tool with a 14 MeV DT source. Slopes of solid black, dashed black, and dotted gray lines identify unity, $\eta$ of far detector, and $\eta$ of near detector, respectively. (c)(d) Similar to (a) and (b), respectively, but for a wireline tool with an AmBe neutron source.

Figure 3.4: Description of FSF and DFD spatial distribution functions for the LWD neutron tool across a limestone block. Top and bottom panels describe far and near detector responses, respectively. (a)(e) Monte Carlo-derived $F S F_{B}$, (b)(f) forward diffusion distributed function, $\psi_{B},(\mathrm{c})(\mathrm{g})$ derivative diffusion distributed function, $J_{B}$, and (d)(h) Rytov DFD function, $J_{B} / \psi_{B}$. Colors describe the relative 2D spatial sensitivity. Solid white lines describe the 1D vertical projection and radial J-factor of relative spatial sensitivities. .79

Figure 3.5: Flow diagram describing the algorithm and implementation of the DFD model for neutron porosity, $\phi_{N}$, simulation .80 
Figure 3.6: Qualitative comparisons of the DFD method to full MCNP calculations across water- and gas-filled limestone formations. Left and right panels show 1D FSF vertical and radial projections, respectively. In this case, the 2D kernel functionality is used for DFD calculations of the LWD tool. (a) Far detector FSF vertical projections, integrated in the radial and azimuthal directions, showing background (limestone block) MC-derived $F S F_{B}$ with a solid black line, MC-derived perturbed $F S F_{M C}$ in homogeneous water- and gas-filled limestone base-cases in solid colored lines, and corresponding DFD perturbed $F S F_{D F D}$ with a dashed blue line. (b) Far detector FSF radial projections, integrated in the vertical and azimuthal directions. (c) Similar to (a) but for the near detector. (d) Similar to (b) but for the near detector......81

Figure 3.7: Quantitative comparisons of 8.5-in-collar LWD $\phi_{N}$ calculated with DFD and LIR methods against MCNP at (a) bottom, B sector orientation, (b) left, L sector orientation, and (c) up, $\mathrm{U}$ sector orientation, in a 12-in. borehole, across various formation base-cases. The schematic diagram above each panel describes the corresponding tool orientation in the borehole. DFD calculations were performed by invoking their 3D kernel functionality. The borehole is assumed to be filled with fresh water. 
Figure 3.8: Qualitative comparisons of 3D FSFs calculated with MCNP, DFD and LIR methods of LWD detector responses at bottom, B sector orientation across a $5 \%$, water-filled limestone formation. The borehole is assumed filled with fresh water. Top and bottom row panels describe FSFs of far and near detectors, respectively. (a)(f) Perturbed detector FSFs using full MCNP calculations, $F S F_{M C}$ and $(\mathrm{b})(\mathrm{g})$ perturbed detector FSFs using the DFD method, $F S F_{D F D}$ viewed at the cross-section $S$ - $S$ ' shown in the borehole schematic describing the corresponding tool orientation in the borehole; 1D FSF projections are identified with solid red lines, i.e., vertical projection, radial J-factor, and azimuthal projection in polar coordinates. (c)(h) 1D FSF radial projections, (d)(i) 1D FSF vertical projections, and (e)(j) 1D FSF azimuthal projections; DFD calculations were performed by invoking their 3D kernel functionality. .83 
Figure 3.9: Qualitative comparisons of $3 \mathrm{D}$ FSFs calculated with MCNP, DFD and LIR methods of LWD detector responses at up, U sector orientation across a 5 $\%$, water-filled limestone formation. The borehole is assumed filled with fresh water. Top and bottom row panels describe FSFs of far and near detectors, respectively. (a)(f) Perturbed detector FSFs using full MCNP calculations, $F S F_{M C}$ and $(\mathrm{b})(\mathrm{g})$ perturbed detector FSFs using the DFD method, $F S F_{D F D}$ viewed at the cross-section $S$ - $S$ ' shown in the borehole schematic describing the corresponding tool orientation in the borehole; 1D FSF projections are identified with solid red lines, i.e., vertical projection, radial J-factor, and azimuthal projection in polar coordinates. (c)(h) 1D FSF radial projections, (d)(i) 1D FSF vertical projections, and (e)(j) 1D FSF azimuthal projections; DFD calculations were performed by invoking their 3D kernel functionality.

Figure 3.10: Comparisons of MCNP, DFD and LIR methods in the presence of fresh water invasion of a gas-filled $30 \%$ limestone formation. Quantitative comparisons of $\phi_{N}$ measurements are shown in the first column, while qualitative comparisons of FSFs are shown in the second and third columns. (a) Simulated far detector counts, (b) simulated near detector counts, (c) simulated $\phi_{N}$ measurements in limestone pu, and (d) $\phi_{N}$ differences versus invasion depth. MCNP, DFD, and LIR measurements are identified with solid red, dashed blue, and solid green lines, respectively. (e) 1D (vertical) far detector FSFs, showing $F S F_{M C}, F S F_{D F D}$, and $F S F_{L I R}$ at various invasion fronts from the borehole wall. (f) Similar to (e) but for the near detector. (g) 1D (radial) far detector FSFs, and (h) 1D (radial) near detector FSFs. The borehole is assumed to be filled with fresh water. .85 
Figure 3.11: Comparisons of LWD neutron porosities $\phi_{N-M C}, \phi_{N-D F D}$, and $\phi_{N-L I R}$, in sandstone pu, simulated with MCNP, DFD, and LIR methods, respectively, in a slick borehole for the synthetic model described in Table 3.3 where TST is $1.3 \mathrm{ft}$. (a) Description of the layered earth model in $L_{m}$ (not to scale), (b) neutron porosity, $\phi_{N-L I R}$ calculated with the LIR method, (c) neutron porosity, $\phi_{N-D F D}$ calculated with the DFD method, (d) neutron porosity, $\phi_{N-}$ ${ }_{M C}$ rendered with full MCNP calculations, (e) compensated density $\rho_{C O-L I R}$ calculated with LIR, (f) bottom sector neutron and density logs (layer descriptions included in Table 3.3), (g) LWD porosity residuals, $\phi_{N-M C}-\phi_{N-}$ $L I R$, (h) LWD porosity residuals, $\phi_{N-M C}-\phi_{N-D F D}$, (i) average error per depth, and (j) average error per azimuth; DFD calculations were performed by invoking their 3D kernel functionality. .86

Figure 3.12: Qualitative comparisons of LWD bottom, B sector FSFs at $15.8 \mathrm{ft}(4.81 \mathrm{~m})$ measured depth across the layered earth model described in Table 3.3. The top row panel describes the LWD tool and layered earth model in $L_{m}$ (not to scale) at this sample depth. Middle and bottom row panels describe FSFs for far and near detectors, respectively. (a)(e) Perturbed detector FSFs rendered with full MCNP calculations, $F S F_{M C}$, (b)(f) perturbed detector FSFs calculated with the DFD method, $F S F_{D F D}$; 1D FSF projections are identified with solid red lines, i.e., vertical projection, radial J-factor, and azimuthal projection in polar coordinates. (c)(g) 1D FSF radial projections, and (d)(h) 1D FSF vertical projections; DFD calculations were performed by invoking their 3D kernel functionality. .87 
Figure 3.13: Qualitative comparisons of LWD bottom, B sector FSFs at $76.0 \mathrm{ft}(23.2 \mathrm{~m})$ measured depth, across the layered earth model described in Table 3.3. The top row panel describes the LWD tool and layered earth model in $L_{m}$ (not to scale) at this sample depth. Middle and bottom row panels describe FSFs for far and near detectors, respectively. (a)(e) Perturbed detector FSFs rendered with full MCNP calculations, $F S F_{M C}$, (b)(f) perturbed detector FSFs calculated with the DFD method, $F S F_{D F D}$; 1D FSF projections are identified with solid red lines, i.e., vertical projection, radial J-factor, and azimuthal projection in polar coordinates. (c)(g) 1D FSF radial projections, and (d)(h) 1D FSF vertical projections; DFD calculations were performed by invoking their 3D kernel functionality.

Figure 3.14: Comparisons of LWD neutron porosities $\phi_{N-M C}, \phi_{N-D F D}$, and $\phi_{N-L I R}$, in sandstone pu, calculated with MCNP, DFD, and LIR methods, respectively, in a slick borehole for the invaded synthetic model described in Table 3.4 where TST is $1.3 \mathrm{ft}$. (a) Description of the layered earth model in $L_{m}$ (not to scale), (b) neutron porosity, $\phi_{N-L I R}$ calculated with the LIR method, (c) neutron porosity, $\phi_{N-D F D}$ calculated with the DFD method, (d) neutron porosity, $\phi_{N-M C}$ rendered with full MCNP calculations, (e) compensated density $\rho_{C O-L I R}$ calculated with LIR, (f) bottom sector neutron and density logs (layer descriptions included in Table 3.4), (g) LWD porosity residuals, $\phi_{N-M C}-\phi_{N-L I R}$, (h) LWD porosity residuals, $\phi_{N-M C}-\phi_{N-D F D}$, (i) average error per depth, and (j) average error per azimuth; DFD calculations were performed by invoking their 3D kernel functionality. .89 
Figure 3.15: Qualitative comparisons of LWD bottom, B sector FSFs at $15.8 \mathrm{ft}(4.81 \mathrm{~m})$ measured depth, across the invaded layered earth model described in Table 3.4. The top row panel describes the LWD tool and layered earth model in $L_{m}$ (not to scale) at this sample depth. Middle and bottom row panels describe FSFs for far and near detectors, respectively. (a)(e) Perturbed detector FSFs rendered with full MCNP calculations, $F S F_{M C}$, (b)(f) perturbed detector FSFs calculated with the DFD method, $F S F_{D F D}$; 1D FSF projections are identified with solid red lines, i.e., vertical projection, radial J-factor, and azimuthal projection in polar coordinates. (c)(g) 1D FSF radial projections, and (d)(h) 1D FSF vertical projections; DFD calculations were performed by invoking their 3D kernel functionality.

Figure 3.16: Qualitative comparisons of LWD bottom, B sector FSFs at $41.6 \mathrm{ft}(12.7 \mathrm{~m})$ measured depth, across the invaded layered earth model described in Table 3.4. The top row panel describes the LWD tool and layered earth model in $L_{m}$ (not to scale) at this sample depth. Middle and bottom row panels describe FSFs for far and near detectors, respectively. (a)(e) Perturbed detector FSFs rendered with full MCNP calculations, $F S F_{M C}$, (b)(f) perturbed detector FSFs calculated with the DFD method, $F S F_{D F D}$; 1D FSF projections are identified with solid red lines, i.e., vertical projection, radial J-factor, and azimuthal projection in polar coordinates. (c)(g) 1D FSF radial projections, and (d)(h) 1D FSF vertical projections; DFD calculations were performed by invoking their 3D kernel functionality. .91

Figure 4.1: Inversion-based workflow for interpretation of LWD nuclear and resistivity measurements acquired in $\mathrm{HA} / \mathrm{HZ}$ wells. 
Figure 4.2: Numerically simulated LWD nuclear and resistivity measurements across a synthetic model. (a) Short-spaced density image, $\rho_{S S}$, (b) long-spaced density image, $\rho_{L S}$, (c) quadrant-sector near neutron count rates, ncps, (d) quadrant-sector far neutron count rates, $f_{c p s}$ in counts per second (cps), (e) quadrant-sector neutron compensated porosity, $\phi_{N}$, (f) GR image, (g) PEF $\log$, and (h) $2 \mathrm{MHz}$ phase-shift array propagation resistivity curves with layer designations. Layer properties are shown in grey stair plots.

Figure 4.3: Layer-based inversion results for the synthetic example, showing inverted (a) bulk density, $\rho_{b}$ with layer designations, (b) migration length, $L_{m}$, (c) shale concentration, $C_{s h}$, (d) photoelectric factor, PEF, and (e) true conductivity, $\sigma_{t}$, in solid blue lines. True synthetic model properties are identified with dashed red lines, respectively, while $95 \%$ inversion confidence intervals are shown with solid green error bars, respectively. (f) Cumulative plots of compositional volumetric concentrations, $V_{n}$ obtained from SNUPAR-based solver and Archie's saturation model. Confidence intervals or uncertainties in compositional volumes, per layer, are shown with black error bars.

Figure 4.4: Comparison of synthetic data and numerically simulated measurements from inverted compositions. (a) Synthetic compensated density data, $\rho_{C O}$, (b) forward simulated compensated density from inverted compositions, (c) percent difference between synthetic and forward simulated $\rho_{C O}$ data, (d) quadrant-sector neutron porosity, $\phi_{N} \operatorname{logs}$, (e) PEF logs, and (f) $2 \mathrm{MHz}$ phase-shift array resistivity curves. Forward simulated curves are identified with dashed black lines. 116 
Figure 4.5: Comparison of inversion-based and conventional interpretation of synthetic LWD nuclear and resistivity measurements acquired in $82^{\circ}$ up-dip well. Cumulative plots of compositional volumetric concentrations, $V_{n}$, obtained from SNUPAR-based solver and Archie's saturation model for (a) inversion-based interpretation, and (b) conventional interpretation. Comparison of calculated (c) total porosity, $\phi_{T}$, (d) water saturation, $S_{w}$, and (e) grain density, $\rho_{g}$. (f) Difference in hydrocarbon pore volume, $H P V$ between inversion-based and conventional interpretation.

Figure 4.6: Effects of bed-geometry uncertainty on layer-based inversion of dualdetector and compensated density and neutron porosity measurements. Descriptions of uncertainty in the geometrical model: From left to right, panels show perturbations in (a) bed boundary location, $B_{b}$, (b) apparent bed dip, $\theta_{b}$, and (c) apparent bed azimuth, $\beta_{b}$. (d) Bulk density, $\rho_{b}$ and (e) migration length, $L_{m}$ from inversion of density and neutron measurements, respectively. Solid blue lines identify true layer-by-layer properties, while blue square points and red circle points identify results obtained from the inversion of dual-detector and compensated measurements, respectively. (f) Porosity difference, in limestone porosity units, due to bed-geometry uncertainty

Figure 4.7: Field case example of LWD nuclear and resistivity measurements in a HA interval. (a) Short-spaced density image, $\rho_{S S}$, (b) long-spaced density image, $\rho_{L S}$, (c) compensated density image, $\rho_{C O}$, showing consolidated gamma borehole sinusoids, (d) bottom sector neutron, $\phi_{N}$, and density, $\phi_{D}$, porosity logs in limestone scale, (e) PEF log, (f) GR log, and (g) 2 MHz phase-shift resistivity curves. 
Figure 4.8: Layer-based inversion results of HA field example, showing inverted (a) bulk density, $\rho_{b}$, (b) migration length, $L_{m}$, (c) photoelectric factor, PEF, (d) shale concentration, $C_{s h}$, and (e) true conductivity, $\sigma_{t}$, in solid blue lines. Predicted properties of SNUPAR-based solver and Poupon-Leveaux saturation model for output compositional volumes are identified with dashed red lines; $95 \%$ inversion confidence intervals are identified with green error bars.

Figure 4.9: Calculated petrophysical properties from inversion results of HA field example. (a) Cumulative plots of compositional volumetric concentrations, $V_{n}$, obtained from SNUPAR-based solver and Poupon-Leveaux saturation model. Confidence intervals or uncertainties in compositional volumes, per layer, are identified with black error bars. Calculated (b) total porosity, $\phi_{T}$, (c) water saturation, $S_{w}$, and (d) grain density, $\rho_{g}$. The $95 \%$ inversion confidence intervals are identified with solid green error bars. (e) Well trajectory across HA interval is identified with a solid red line and apparent bed orientation is identified with tadpole-like points, i.e., bed dip and azimuth

Figure 4.10: Field case example of LWD nuclear and resistivity measurements in a $\mathrm{HZ}$ interval. (a) Compensated density image, $\rho_{C O}$, showing gamma borehole sinusoids, (b) 2 MHz phase-shift resistivity curves, (c) GR log, (d) PEF log, (e) bottom sector neutron, $\phi_{N}$, and density, $\phi_{D}$, porosity logs in limestone scale, and (f) 2D curtain section diagram of layer-based geometry and well trajectory (not to scale). 
Figure 4.11: Layer-based inversion results of HZ field example, showing inverted (a) bulk density, $\rho_{b}$, (b) migration length, $L_{m}$, (c) photoelectric factor, PEF, (d) shale concentration, $C_{s h}$, and (e) true conductivity, $\sigma_{t}$, in solid blue lines. Predicted properties of SNUPAR-based solver and Poupon-Leveaux saturation model for output compositional volumes are identified with dashed red lines; $95 \%$ inversion confidence intervals are identified with green error bars.

Figure 4.12: Comparison of inversion-based and conventional interpretation results of HZ field example. Cumulative plots of compositional volumetric concentrations, $V_{n}$, obtained from SNUPAR-based solver and PouponLeveaux saturation model for (a) inversion-based interpretation, and (b) conventional interpretation. Comparison of (c) total porosity, $\phi_{T}$, (d) water saturation, $S_{w}$, and (e) grain density, $\rho_{g}$. (f) Difference in hydrocarbon pore volume, $H P V$, between inversion-based and conventional interpretation. (g) Well trajectory across $\mathrm{HZ}$ interval is identified with a solid red line and apparent bed orientation is identified with tadpole-like points, i.e., bed dip and azimuth.

Figure 4.13: Qualitative comparison of forward simulated measurements, from inverted compositions, and HZ field data. (a) Field compensated density data, $\rho_{C O}$, and (b) forward simulated compensated density from inverted compositions. Field and forward simulated (c) neutron porosity, $\phi_{N}$, (d) GR, and (e) PEF logs. Field and forward simulated logs are identified with solid red and dashed blue lines, respectively. (f) $2 \mathrm{MHz}$ phase-shift array resistivity curves, field and forward simulated curves are identified with solid and dashed colored lines, respectively. 
Figure 5.1: SNUPAR-calculated water-filled neutron porosity calibration functions $g_{s}$, $g_{l}$, and $g_{d}$ for sandstone, limestone, and dolomite units, respectively. The figure also shows neutron porosity responses across relevant formations. 148

Figure 5.2: Interactive interpretation workflow for interpretation of neutron and density apparent porosities

Figure 5.3: Simulated well logs across the synthetic multi-layer model. (a) Gamma ray (GR) log, (b) neutron and density apparent porosities on a limestone scale, (c) array induction apparent resistivity logs, and (d) PEF log. Refer to Table 5.1 for a description of assumed layer properties. 150

Figure 5.4: Interpretation results for the Synthetic Example using the interactive interpretation workflow. (a) Interpreted matrix density from SNUPARbased matrix solver, (b) neutron-density overlay showing shale-corrected neutron log, matrix and fluid crossover characteristics, (c) neutron and density apparent water-filled logs from SNUPAR-based matrix solver, (d) interactive flag indicators showing matrix effect and gas flag, (e) corrected neutron-density overlay, (f) estimated total porosity, (g) estimated water saturation, and (h) estimated hydrocarbon and fluid densities. Refer to Table 5.1 for a description of layer properties. 
Figure 5.5: Interpretation results for Field Example I, gas-bearing carbonate reservoir, using the interactive interpretation workflow. (a) Gamma ray log, (b) neutron and density porosities on limestone scale, (c) dual-induction resistivity logs, and (d) photoelectric factor log. (e) Matrix density, (f) total porosity, and (g) water saturation from core measurements and interactive analysis. (h) Calculated fluid densities showing a gas cut-off of $0.25 \mathrm{~g} / \mathrm{cm}^{3}$. (i) Volumetric concentrations of rock and fluid components from SNUPARbased solver. (j) Gas flag from interactive analysis workflow. 152

Figure 5.6: Interpretation results for Field Example II, oil-bearing shale reservoir, using the interactive analysis workflow. (a) Gamma ray log, (b) neutron and density porosities on limestone scale, (c) array induction resistivity logs, and (d) photoelectric factor log. (e) Matrix density, (f) total porosity, and (g) water saturation from core measurements and interactive analysis. (h) Calculated fluid densities showing a gas cut-off of $0.25 \mathrm{~g} / \mathrm{cm}^{3}$..... 153

Figure 5.7: Fluid zone interpretation results for Field Example III, North Sea siliciclastic reservoir. (a) Gamma ray log, (b) photoelectric factor log, (c) neutron and density porosities on limestone scale, and (d) array induction resistivity logs. (e) Estimated water saturation, (f) pressure measurements, and (g) fluid densities from interactive analysis and pressure gradients. (h) Total porosity from core measurements and interactive analysis.

Figure 5.8: Fluid zone interpretation results for Field Example IV, deepwater Gulf of Mexico reservoir. (a) Gamma ray log, (b) photoelectric factor log, (c) neutron and density porosities on sandstone scale, and (d) array induction resistivity logs. (e) Total porosity, (f) water saturation, and (g) fluid density logs estimated using the interactive analysis. 
Figure 6.1: Flow chart summarizing the three parts of the dissertation. ARC: Schlumberger Array Resistivity Compensation measurements; SNUPAR: Schlumberger Nuclear Parameter program.

Figure 6.2: Preliminary results toward the development of a gamma flux-difference approximation method for Compton scattering. The y-axis describes the modified gamma parameter (analogous to modified neutron travel length discussed in Chapter 3) for short- (SS) and long-spaced (LS) detectors, while the $\mathrm{x}$-axis describes the formation bulk density; SS and LS detector trend lines indicate constant gamma coefficients for sandstone, limestone, and dolomite formations, including aluminum and magnesium calibration blocks. 175

Figure A.1: Comparisons of LWD neutron porosities $\phi_{N-M C}, \phi_{N-D F D}$, and $\phi_{N-L I R}$, in sandstone pu, simulated with MCNP, DFD, and LIR methods, respectively, in a 12-in. water-filled borehole for the synthetic model described in Table 3.3 where TST is $1.3 \mathrm{ft}(40 \mathrm{~cm})$. (a) Description of the layered earth model in $L_{m}$ (not to scale), (b) neutron porosity, $\phi_{N-L I R}$ calculated with the LIR method, (c) neutron porosity, $\phi_{N-D F D}$ calculated with the DFD method, (d) neutron porosity, $\phi_{N-M C}$ rendered with full MCNP calculations, (e) compensated density $\rho_{C O-L I R}$ calculated with LIR, (f) bottom sector neutron and density logs (layer descriptions included in Table 3.3), (g) LWD porosity residuals, $\phi_{N-M C}-\phi_{N-L I R}$, (h) LWD porosity residuals, $\phi_{N-M C}-\phi_{N-}$ DFD, (i) average error per depth, and (j) average error per azimuth; DFD calculations were performed by invoking their 3D kernel functionality. ...179 
Figure A.2: Qualitative comparisons of the DFD method to full MCNP calculations across water- and gas-filled limestone formations, in the presence of 2-in. standoff for wireline sonde. Left and right panels show 1D FSF vertical and radial projections, respectively. In this case, the $2 \mathrm{D}$ kernel functionality is used for DFD calculations of the wireline tool. (a) Far detector FSF vertical projections, integrated in the radial and azimuthal directions, showing background MC-derived $F S F_{B}$ with solid black lines, MC-derived perturbed $F S F_{M C}$ in solid colored lines, corresponding DFD perturbed $F S F_{D F D}$ with a dashed blue line, and equivalent $F S F_{L I R}$ with solid green lines. (b) Far detector FSF radial projections, integrated in the vertical and azimuthal directions, showing the standoff region. (c) Similar to (a) but for the near detector. (d) Similar to (b) but for the near detector. The borehole is assumed to be filled with fresh water.

Figure A.3: Quantitative comparisons of wireline $\phi_{N}$ calculated with DFD and LIR methods against MCNP. (a) Error in far and near detector counts versus formation $\phi_{N}$ in a 12-in. borehole. (b) $\phi_{N}$ errors in a 12 -in. borehole. (c) $\phi_{N}$ errors in 10-in. and 15-in. boreholes. DFD calculations were performed by invoking their 3D kernel functionality. The borehole is assumed to be filled with fresh water. 
Figure A.4: Comparisons of LWD neutron porosities $\phi_{N-M C}, \phi_{N-D F D}$, and $\phi_{N-L I R}$, in sandstone pu, simulated with MCNP, DFD, and LIR methods, respectively, in a slick borehole for the synthetic model described in Table 3.3 where TST is $0.49 \mathrm{ft}(15 \mathrm{~cm})$. (a) Description of the layered earth model in $L_{m}$ (not to scale), (b) neutron porosity, $\phi_{N-L I R}$ calculated with the LIR method, (c) neutron porosity, $\phi_{N-D F D}$ calculated with the DFD method, (d) neutron porosity, $\phi_{N-M C}$ rendered with full MCNP calculations, (e) compensated density $\rho_{C O-L I R}$ calculated with LIR, (f) bottom sector neutron and density logs (layer descriptions included in Table 3.3), (g) LWD porosity residuals, $\phi_{N-M C}-\phi_{N-L I R}$, (h) LWD porosity residuals, $\phi_{N-M C}-\phi_{N-D F D}$, (i) average error per depth, and (j) average error per azimuth; DFD calculations were performed by invoking their 3D kernel functionality.

Figure C.1: Effects of resistivity anisotropy and polarization horns on inversion of propagation resistivity measurements simulated across synthetic model described in Table C.1. (a) $2 \mathrm{MHz}$, (b) $400 \mathrm{kHz}$ phase-shift and attenuation array resistivity measurements; (c) inverted layer-by-layer vertical, horizontal, and calculated true resistivities, (d) resistivity confidence intervals, and (e) layer-by-layer resistivity anisotropy coefficient. 
Figure C.2: Estimation of formation anisotropy across HA interval in field case example. (a) Field data compensated density image showing consolidated bed boundaries. Field and numerically simulated (b) neutron porosity, and (c) GR measurements. Field and numerically simulated array, 16- and 40in., propagation resistivity measurements, showing (d) $400 \mathrm{kHz}$, and (e) 2 $\mathrm{MHz}$ frequency curves. (f) Inverted layer-by-layer vertical, horizontal resistivities; and calculated true resistivity. (g) Calculated layer-by-layer

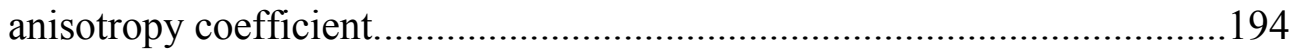




\section{Chapter 1: Introduction}

This dissertation introduces new methods for quantitative petrophysical interpretation of logging-while-drilling nuclear and resistivity measurements based on borehole measurement physics and inverse theory.

First, as a secondary objective, I develop new numerical simulation techniques for accurate forward modeling of neutron porosity measurements in the presence of borehole,

environmental, and bed-geometry effects. Second, as the main objective of the dissertation, algorithms are introduced for nonlinear petrophysical and compositional inversion of logging-while-drilling multi-sector nuclear and multi-array propagation resistivity measurements acquired in highly-deviated and horizontal wells. Finally, using the compositional inversion algorithm for a supplementary objective, this dissertation introduces an interactive well-log interpretation method involving matrix analysis of neutron and density measurements. The latter interpretation workflow estimates hydrocarbon densities and identifies fluid zones.

\subsection{BACKGROUND}

High-angle and horizontal (HA/HZ) wells are drilled to maximize reservoir exposure, hence improve hydrocarbon production. Because attaining the desired well placement targets while drilling is a challenging task, $\mathrm{HA} / \mathrm{HZ}$ wells commonly undulate through pay and non-pay reservoir zones. This behavior gives rise to adverse conditions and challenging well-log features such as polarization horns, anisotropy in apparent resistivity, and "bull's-eye" features (refer to Figure 1.1) on nuclear borehole images. For this reason, traditional well-log interpretation methods often produce unreliable results in $\mathrm{HA} / \mathrm{HZ}$ wells. Understanding $\mathrm{HA} / \mathrm{HZ}$ geometry effects on well-log 
measurements is critical for reliable formation evaluation in highly-deviated wells (Passey et al., 2005; Rendeiro et al., 2005).

Better insights to well-log response behavior and advancements in interpretation can be achieved through forward modeling of logging-while-drilling (LWD) multi-sector nuclear measurements (Uzoh et al., 2009; Mendoza et al., 2010c; Ijasan et al., 2011). These forward models are important requirements for development of inversion-based interpretation techniques. Extension of LWD modeling capabilities to sector-based inversion of density logs (Mendoza et al., 2012; Shetty et al., 2012) was shown to improve estimation of bed density. No published documents exist on the integration of multi-sector LWD neutron, density, gamma ray (GR), photoelectric, and array resistivity measurements for petrophysical assessment in $\mathrm{HA} / \mathrm{HZ}$ wells.

Traditionally, because the Monte Carlo N-Particle program (MCNP, X-5 Monte Carlo Team, 2003) efficiently takes into account complex tool and formation geometries, it has been applied for forward modeling of borehole nuclear measurements. The program stochastically solves the Boltzmann transport equation by tracking millions of particles from source to detectors in three-dimensional (3D) space as they undergo several transport processes. For this reason, the method is computationally expensive. Even though variance reduction techniques (Booth et al., 1984) are applied to increase Monte Carlo (MC) calculation efficiencies, MC methods are still inefficient for simulating borehole measurements over standard logging depth intervals. With the MCNP code, a typical LWD multi-sector nuclear image could take days or weeks to simulate on a desktop computer. This makes the algorithm inefficient for well-log inversion applications.

By modifying the MCNP code to derive differential sensitivity functions (Watson, 1984; 1992; Couët et al., 1993; Case et al., 1994) from adjoint function solutions, new 
opportunities arose for development of fast-forward modeling techniques suited for nuclear well-log inversion. Using perturbation theory and linear first-order approximations (Greenspan, 1976; Lewins, 1965), the differential flux sensitivity function (FSF) technique has been widely applied for fast and interactive numerical modeling of borehole wireline and LWD nuclear measurements (Mendoza et al., 2007; Heidari et al., 2009; Ijasan et al., 2011). But due to significant neutron perturbation effects, first-order approximations for modeling borehole neutron measurements tend to yield inaccurate results across dissimilar regions with large contrasts in neutron energy cross-section or/and hydrogen index (HI). These perturbation effects manifest themselves as higher-order terms in the Taylor's series expansion of the differential operator perturbation function (X-5 Monte Carlo Team, 2003). A diffusion correction method for simulating Sigma measurements in the presence of such perturbations was shown to improve accuracy across layers of dissimilar capture cross-sections (Mimoun et al., 2011). Methods that incorporate higher-order dependencies on borehole and environmental effects have also shown to improve accuracy in simulating gamma-gamma measurements (density and photoelectric factor) acquired in the presence of mudcake (Zhou et al., 2009). For borehole applications, no published work exists on diffusion or/and higher-order sensitivity analysis for accurate and efficient numerical modeling of neutron-neutron measurements.

Recent advances in fast-forward simulation of nuclear measurements (Mendoza et al., 2007; 2010a; 2010b) have encouraged the development of linear and nonlinear inversion-based interpretation algorithms that combine nuclear, resistivity, and acoustic measurements (Heidari et al., 2009; Mallan et al., 2009). In well-log interpretation, inversion techniques are the only means to account for measurement variations due to simultaneous effects of formation petrophysical and geometrical properties. Several 
studies have shown that joint inversion algorithms that integrate several borehole measurements in vertical wells improve layer-by-layer petrophysical assessment of hydrocarbon reservoirs across thinly-bedded formations (Liu et al., 2007; Heidari et al., 2009; Sanchez-Ramirez et al., 2010).

In the past, inverse theory has been useful to calculate resistivity properties from borehole induction and propagation electromagnetic measurements. These inversion methods are applied in conjunction with conventional nonlinear saturation models, e.g., Archie's equation for clean formations and dual-water model for shaly formations (Ellis et al., 2007). Nonlinear gradient-based inversion techniques, such as Gauss-Newton and Levenberg-Marquardt minimization methods (Hansen, 1998; Aster et al., 2005), are implemented for inversion of apparent resistivity measurements because the resistivity response is often nonlinear with respect to formation properties. These techniques are used to estimate radial saturation profiles in the presence of mud-filtrate invasion (Mallan et al., 2009), assess the effects of thin beds and laminations on resistivity logs (Bootle et al., 2009), and investigate the effects of bed anisotropy and polarization horns due to highly dipping beds (Chemali et al., 1987; Hagiwara, 1997; Guzmán-Garcia, 2002).

\subsection{Problem Statement}

The application of interpretation methods developed for vertical wells in HA/HZ wells often under-estimates hydrocarbon pore volume (HPV) in hydrocarbon-bearing reservoirs. Understanding the physical relationships that govern how bed geometrical properties affect LWD measurement responses can contribute to the development of new and improved formation evaluation practices specific for $\mathrm{HA} / \mathrm{HZ}$ wells.

Figure 1.1 shows a field example of an azimuthal LWD compensated density image acquired in an undulating $\mathrm{HA} / \mathrm{HZ}$ well. The left panel describes the field 
measurement with interpreted bed boundaries shown with solid white lines, while the right panel describes the well trajectory and bed geometrical properties. Due to imperfections in well placement and an undulating well trajectory, high and low density layers are simultaneously sampled in the same azimuthal measured depth. This sampling gives rise to the "bull's-eye" feature from ZY35 - ZY50 ft in the density image indicated in the left panel of Figure 1.1. Conventional interpretation developed for vertical wells makes several assumptions. For example, azimuthal homogeneity in the borehole, i.e. logs at the same measured depth sample the same petrophysical layer. Applying this assumption to the measurements in Figure 1.1 would be equivalent to depth-by-depth averaging of both the high and low density layers. Such an approach will obviously degrade the accuracy of interpreted formation porosity, and consequently, of hydrocarbon pore volume.

The problem is further complicated across thinly bedded formations and in the presence of 3D asymmetric invasion resulting from gravity segregation in $\mathrm{HA} / \mathrm{HZ}$ wells. Even though invasion is assumed to be marginal during LWD measurement acquisition, nuclear and resistivity responses still measure different degrees of invaded and in-situ formation properties. Furthermore, resistivity measurements exhibit polarization horns when induced eddy currents are forced across dipping formation layers of dissimilar electrical properties. Such an artifact becomes more pronounced with increasing values of relative bed dip and resistivity contrast. It is for all these reasons that conventional petrophysical interpretation methods used in vertical wells can yield inaccurate results in $\mathrm{HA} / \mathrm{HZ}$ wells.

The physical relationships in $\mathrm{HA} / \mathrm{HZ}$ wells governing how bed geometrical properties affect LWD measurements are complex. The interdependent factors contributing to this complexity include measurement value, bed boundary detection, 
separation of array resistivity measurements, differing volumes of investigation, petrophysical properties, bed dip, bed azimuth, well inclination, and tool orientation. Modeling-based interpretation is the most reliable method to simultaneously account for all these physical relationships because they are described by the corresponding nuclear or resistivity measurement physics, i.e., tool design, source-detectors configuration, and transmitter-receivers configuration. Therefore, an essential prerequisite for petrophysical inversion is fast, accurate, and efficient numerical simulation of borehole measurements.

Differential flux sensitivity techniques that employ perturbation theory have proven to be suitable for fast and interactive nuclear measurement modeling. These methods implement first-order perturbation approximations and a linear iterative refinement of three-dimensional (3D) spatial flux sensitivity functions (FSFs) precalculated in homogeneous formations. The FSFs are numerical solutions to the integrodifferential adjoint transport equation. However, it has been found that first-order calculations can yield inaccurate results when simulating neutron porosity measurements (a) across invaded gas-saturated formations, (b) across highly deviated beds with formation layers of significant HI contrast, and (c) in the presence of LWD azimuthallyvarying standoff. A similar problem is observed in the numerical simulation of Sigma measurements across bed boundaries between sands and shales. Due to significant variability or contrasts in borehole or/and formation neutron energy cross-sections, diffusion effects can render linear first-order assumptions inaccurate to describe differential changes in neutron porosity measurements simulated with the linear iterative refinement technique.

Because of gravity in $\mathrm{HA} / \mathrm{HZ}$ wells, the LWD tool collar-stabilizer assembly rests at the bottom of the borehole such that minimum and maximum tool standoffs occur at the bottom and top of the borehole, respectively. In order words, the tool is eccentered in 
the borehole. This behavior results in an azimuthally-varying standoff around the hole. Consequently, neutron measurements binned at the top azimuths are more affected by standoff and diffusion effects than measurements binned at the bottom azimuths whereby they are more difficult to accurately model with the FSF technique. An approach to circumvent the modeling problem is to pre-calculate FSFs in the various azimuths as the tool rotates in the borehole, at the expense of more computation time and larger storage disk space. A more efficient approach is to augment pre-calculated MC-derived FSFs with fast semi-analytical models that calculate spatial neutron flux perturbations in the presence of such azimuthally-varying standoff. With this latter approach, petrophysical and geometrical models descriptive of neutron porosity measurements acquired in the presence of azimuthally-varying standoff across desirable reservoir zones can enhance the assessment of reservoir capacity.

Estimation of total porosity from neutron and density logs is still the most common way to assess reservoir capacity. Matrix and fluid effects on well-logs are directly related to formation matrix and fluid densities; hence, estimating porosity from density logs is straightforward using volumetric calculations if the matrix and fluid densities are known beforehand. On the other hand, matrix and fluid effects on neutron logs are further influenced by shale type, matrix-hydrogen, and hydrocarbon type, whereby the interpretation of neutron logs is not trivial, especially across mixed/complex lithologies and shaly formations. Additionally, neutron porosity logs are customarily delivered on a pre-defined matrix scale such that special corrections or rescaling methods are necessary when the chosen matrix scale differs from that of the in-situ formation. It is essential that porosity estimated from neutron and density logs be independent of formation lithology or matrix scale. Misinterpreting total porosity by a few percent could lead to misidentification of hydrocarbon type (gas or oil). Few publications discuss 
model-consistent methods that interpret matrix and fluid effects from conventional welllogs for estimation of lithology-independent porosity.

\subsection{RESEARCH OBJECTIVES}

The main thrust of the dissertation is to develop quantitative interpretation methods that explicitly account for geometrical effects in $\mathrm{HA} / \mathrm{HZ}$ wells for integration of LWD multi-sector nuclear and multi-array resistivity measurements. Specifically, the objectives of the dissertation are as follows:

- To develop an algorithm for integrated petrophysical, compositional, and geometrical interpretation of LWD density, neutron porosity, photoelectric factor (PEF), natural gamma ray (GR), and apparent resistivity measurements acquired in $\mathrm{HA} / \mathrm{HZ}$ wells. To achieve this objective, inversion techniques in conjunction with multi-physics 3D forward models are implemented for processing multisector nuclear and multi-array resistivity measurements under the assumption of multi-layer formation models.

- To develop a neutron porosity parameter, in lieu of neutron energy cross-section and migration length, from relevant petrophysical properties for application in the inversion algorithm. In conjunction with the FSF linear iterative refinement technique, the new parameter is to be implemented for fast-forward modeling of multi-sector LWD neutron porosity measurements in the presence of borehole environmental effects. The fast-forward model with the new parameter is intended to reproduce neutron detector responses across formations of various saturating fluids, including water of varying salinity and gas of varying density.

- $\quad$ To develop a fast technique that calculates neutron (wireline and LWD) FSF perturbations due to higher-order transport effects for application in the inversion 
algorithm. The new calculation technique will be particularly suited for efficient and reliable simulation of neutron porosity logs in the presence of azimuthallyvarying standoff, mud-filtrate invasion, and across highly dipping beds with significant bed property contrasts. To that end, a modified neutron diffusion model is implemented to calculate FSF perturbations, hence account for higherorder perturbation effects. Additionally, the technique aims to circumvent precalculating FSFs for several borehole sizes and environmental conditions.

- $\quad$ To compare the two newly introduced neutron porosity fast-forward modeling techniques and select the more reliable and robust approach to interpret LWD neutron porosity measurements in the inversion algorithm.

- To develop a multi-mineral compositional solver via nonlinear numerical inversion for volumetric interpretation of rock and fluid constituents from logderived or/and inverted formation petrophysical parameters such as density, migration length, photoelectric factor, GR, and resistivity.

- To verify the reliability of the developed inversion techniques for improved formation evaluation using challenging synthetic and field examples of LWD nuclear and resistivity measurements acquired in $\mathrm{HA} / \mathrm{HZ}$ wells.

- $\quad$ To verify the developed inversion-based compositional multi-mineral solver for interactive matrix analysis of depth-by-depth density and neutron porosity measurements in conjunction with other well logs for assessment of formation porosity and hydrocarbon density.

\subsection{METHOD OVERVIEW}

In the first part of this dissertation, I develop two new methods for accurate fastforward simulation of wireline single-sector and LWD multi-sector neutron porosity 
measurements. The first method introduces a new neutron-density formation parameter for modeling neutron porosity measurements across formations with varying petrophysical properties, including borehole environmental effects (e.g., tool standoff and mud-filtrate invasion). The parameter is obtained by nonlinear regression of the MCderived neutron far and near detector responses in various formation bases-cases with a petrophysical model that combines neutron characteristic lengths and bulk density. For fast-forward modeling, the new formation parameter is implemented with the FSF linear iterative refinement technique. The fast simulation method is benchmarked with MCderived neutron porosity measurements and several LWD tool stabilizer and borehole sizes. Additionally, I validate the fast-forward model with experimental neutron porosity measurements for a specific commercial LWD tool.

The second method implements one-group 2D and 3D diffusion models as Green's function kernels for approximating neutron flux perturbations, higher-order transport effects, and variability in depth-by-depth neutron porosity measurements. Next, transport corrections modify the diffusion model and a flux-difference calculation estimates higher-order terms in the Taylor's series expansion of the detector response perturbation function. I enforce flux continuity conditions at boundaries between dissimilar formation properties arising from mud-filtrate invasion or/and tool standoff. The technique is benchmarked with full 3D calculations of MCNP-derived spatial FSFs and neutron porosity measurements acquired with wireline and LWD tools in the presence of highly dipping beds, mud-filtrate invasion, and/or tool standoff. Comparisons of computational speed and accuracy are made between the flux-difference approximation and the linear iterative refinement method. Furthermore, this part of the dissertation examines the two introduced methods for numerically simulating neutron 
porosity logs to select the more accurate and efficient forward modeling technique for inversion of neutron porosity measurements.

The second part comprises the main contribution from the dissertation and introduces an algorithm for inversion-based interpretation of LWD nuclear and resistivity measurements acquired in $\mathrm{HA} / \mathrm{HZ}$ wells. Using a three-step workflow, the algorithm implements the corresponding property parameterization and data domain for each nuclear and resistivity measurement depending on sector-binning, detector compensation, and array channels. The first step involves interpretation of layer-based geometrical properties and bed boundary selection from sector-based density, GR, and PEF measurements. Selected boundaries consider each measurement's radial length of investigation and are combined for inferring bed boundary sinusoids, relative bed dip, and relative bed strike. The second step implements regularized nonlinear minimization of a quadratic error cost function between the data and numerically simulated measurements within each domain. Starting with an initial guess of layer-by-layer properties, I implement gradient-based separate nonlinear inversion of multi-sector nuclear and multi-array propagation resistivity measurements. Here, the 3D measurement physics in each domain is described with appropriate fast-forward models and Jacobian operator matrices. Numerical entries of Jacobian matrices in the nuclear measurement domain are constructed from 3D FSFs and rapid simulation of detector compensated measurements. Additionally, for neutron porosity measurements, the FSFs are augmented with the newly introduced neutron diffusion flux-difference approximation. In the final step of the inversion-based workflow, layer-by-layer rock and fluid constituents are estimated from previously inverted layer-by-layer properties using a physics-based multimineral compositional rock/fluid solver. The multi-mineral solver implements relationships among inverted properties and rock/fluid mixtures which are explicitly 
calculated using Schlumberger's Nuclear Parameter program (SNUPAR, McKeon et al., 1989). The SNUPAR program calculates nuclear parameters such as neutron characteristic lengths, photoelectric factor, Sigma, bulk density, etc., for complex rock and fluid mixtures. Using synthetic and field examples, I compare estimated hydrocarbon pore volumes obtained with the inversion-based workflow to calculations performed with conventional interpretation techniques.

In the final part of the dissertation, I implement the SNUPAR-based compositional rock/fluid solver for interactive matrix analysis of depth-by-depth neutron and density porosity logs acquired in vertical wells. This solver interprets matrix effects from nuclear logs and defines an interactive, variable neutron-density matrix scale. The new interactive scale updates the neutron-density overlay such that fluid effects are enhanced for assessment of formation porosity, estimation of hydrocarbon density, and identification of hydrocarbon-saturated depth intervals. For validation, I verify the technique on several field examples and compare results to laboratory core measurements.

\subsection{OUTLine OF THE DisSERTATiON}

This dissertation consists of three parts straddling six chapters. Following the introductory chapter, the first part of the dissertation is described in Chapters 2 and 3. Chapter 2 develops and validates a new formation parameter in conjunction with the FSF linear iterative refinement technique for numerical simulation of neutron porosity measurements. Subsequently, Chapter 3 documents the successful development and application of a new diffusion flux-difference approximation for rapid calculation of higher-order effects in neutron porosity measurements. The main contribution is the improvement in accuracy and computational efficiency for forward and inverse modeling 
of neutron porosity measurements. Chapter 3 also compares the two fast simulation methods described in the first part of the dissertation.

Chapter 4 discusses the second part and main objective of the dissertation, i.e. implementation of the inversion-based algorithm for combined interpretation of LWD nuclear and resistivity measurements acquired in highly-deviated and horizontal wells. The main contributions are the integration of multi-physics measurement models, explicit correction of bed-geometry and well trajectory effects, and consolidation of formation structural properties inferred from multiple gamma borehole measurements.

Chapter 5 describes the third part and supplementary objective of the dissertation, i.e. the use of the multi-mineral rock/fluid solver to develop a variable and interactive matrix scale for adaptive correction of neutron and density porosity logs in vertical wells. The main contribution of this part of the dissertation is the identification of hydrocarbon types from conventional well logs.

Finally, Chapter 6 summarizes the contributions, conclusions, and future research recommendations stemming from the dissertation.

\subsection{List OF Publications}

Several journal and conference papers based on the research documented in this dissertation have been published or submitted for review. They are listed below.

\subsubsection{Refereed Journal Publications}

Ijasan, O., C. Torres-Verdín, and W. E. Preeg, 2013, Fast modeling of borehole neutron porosity measurements with a new spatial transport-diffusion approximation: Geophysics, 78, no. 3, D151-D168.

Ijasan, O., C. Torres-Verdín, and W. E. Preeg, 2013, Inversion-based petrophysical interpretation of logging-while-drilling nuclear and resistivity measurements: Geophysics (accepted for publication). 
Ijasan, O., C. Torres-Verdín, and W. E. Preeg, 2013, Interpretation of porosity and fluid constituents from well-logs using an interactive neutron-density matrix scale: Interpretation (accepted for publication).

Ijasan, O., C. Torres-Verdín, W. E. Preeg, J. Rasmus, and E. Stockhausen, 2013, Field examples of the joint petrophysical inversion of logging-while-drilling nuclear and resistivity measurements acquired in high-angle and horizontal wells: Geophysics (to be submitted for review).

\subsubsection{Refereed Conference Proceedings}

Ijasan, O., C. Torres-Verdín, W. E. Preeg, and A. Mendoza, 2011, Rapid modeling of LWD nuclear measurements acquired in high-angle and horizontal wells for improved petrophysical and geometrical interpretation: Transactions of the Society of Petrophysicists and Well Log Analysts, 52nd Annual Logging Symposium, paper R, Colorado Springs, Colorado, May 14-18.

Ijasan, O., C. Torres-Verdín, and W. E. Preeg, 2012, Fast modeling of borehole neutron measurements with a new transport-diffusion approximation: Society of Exploration Geophysicist International Exposition and 82nd Annual Meeting, Las Vegas, Nevada, November 4-9.

Ijasan, O., C. Torres-Verdín, and W. Preeg, 2013, Estimation of porosity and fluid constituents from neutron and density logs using an interactive matrix scale: Transactions of the Society of Petrophysicists and Well Log Analysts, 54th Annual Logging Symposium, paper T, New Orleans, Louisiana, June 22-26.

Ijasan, O., C. R. Case, J. M. Chiaramonte, D. V. Ellis, and B. A. Roscoe, 2013, Fastforward modeling of azimuthal logging-while-drilling neutron porosity measurements in the presence of borehole and environmental effects: Transactions of the Society of Petrophysicists and Well Log Analysts, 54th Annual Logging Symposium, paper WW, New Orleans, Louisiana, June 22-26.

Rasmus, J., O. Ijasan, C. Morriss, R. Griffiths, D. V. Ellis, J. M. Chiaramonte, C. R. Case, and C. Torres-Verdín, 2013, Is porosity anisotropy real and will it affect my petrophysical evaluations?: Transactions of the Society of Petrophysicists and Well Log Analysts, 54th Annual Logging Symposium, paper K, New Orleans, Louisiana, June 22-26. 
(a)

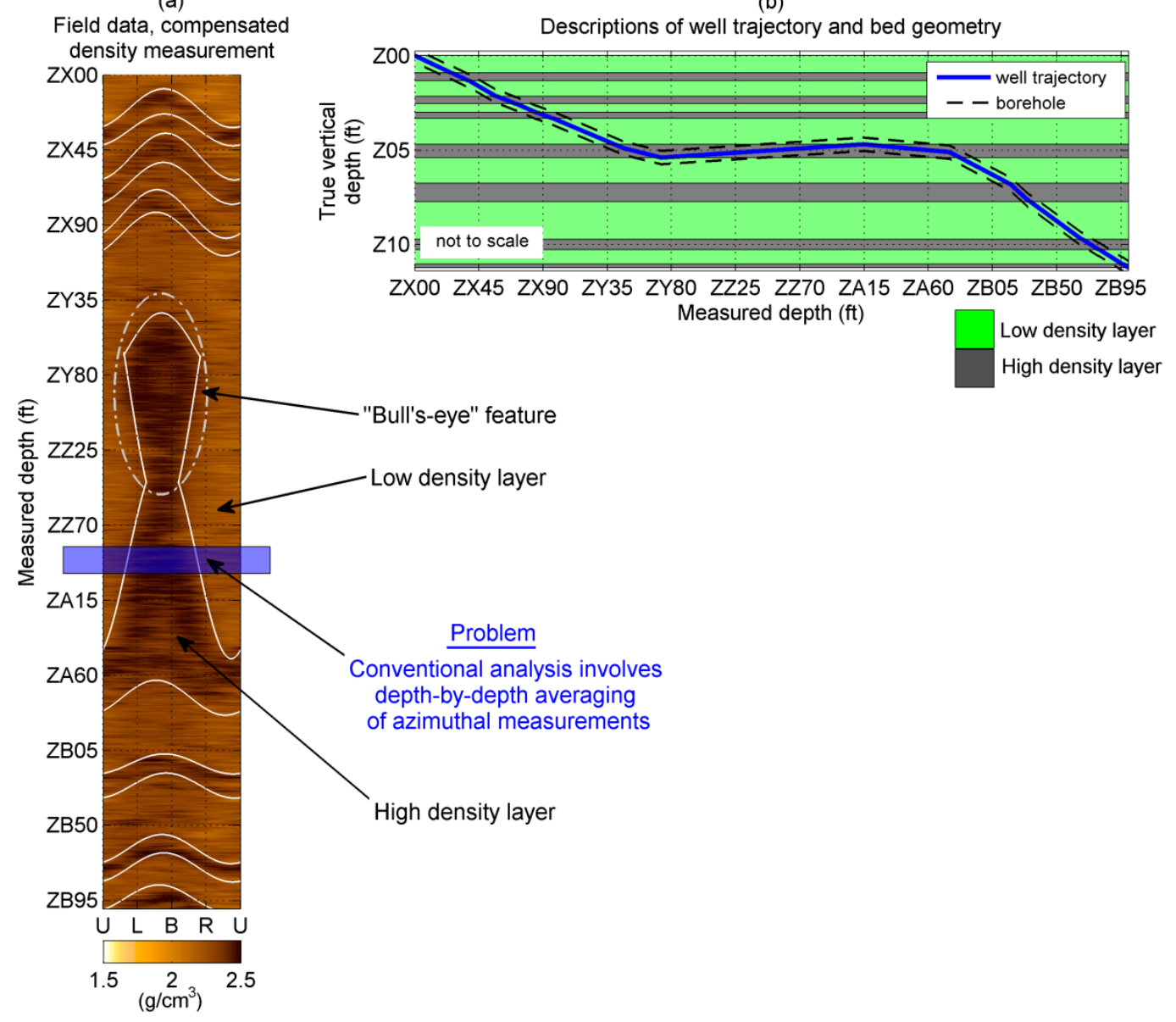

Figure 1.1: Field example of undulating well trajectory effects in LWD compensated density measurements. (a) Compensated density image showing high and low density layers, and the characteristic "bull's-eye" feature in undulating $\mathrm{HA} / \mathrm{HZ}$ wells. (b) Description of well trajectory and formation geometrical properties. 


\section{Chapter 2: Fast-Forward Modeling of Logging-While-Drilling Neutron Porosity Measurements in the Presence of Borehole and Environmental Effects}

This chapter introduces the first simulation method in the first part of the dissertation. It describes a new technique for fast numerical simulation of azimuthal neutron porosity measurements in the presence of borehole and environmental effects.

Numerical and computational methods for solving the elegant Boltzmann transport equation (BTE), or its approximations, include Monte Carlo (MC), discrete ordinates, and diffusion solutions. These methods, of varying accuracy, are laborious and computationally inefficient for modeling and inversion of borehole nuclear measurements. The concept of adjoint solutions or flux sensitivity functions (FSFs) for estimating detector sensitivities, resulting from formation and borehole perturbations, has proven to be both an accurate and fast method for simulating and understanding nuclear logs. Obtaining a petrophysically significant formation parameter, in lieu of energydependent nuclear cross sections, for perturbation estimates could prove to be challenging, considering the complexity of particle transport physics. This calculated formation parameter must be robust to account for petrophysical, geometrical, and borehole environmental effects. In gamma-gamma tools, electron density and photoelectric factor govern the basis of bulk density and photoelectric measurements with a linear relationship. Migration length is an important formation property tightly related to the measurement principle of neutron porosity responses. But as formation lithology and fluid compositions (e.g., formation gas density) vary, the relationship between neutron responses and migration length becomes highly nonlinear. In the presence of borehole and environmental effects, response perturbations increase, further complicating the nonlinearity. 
The choice of formation parameter becomes crucial in generalizing detector responses to petrophysical and environmental effects for accurate and robust fast-forward modeling of nuclear logs. The inverse of migration length, as the formation parameter, has shown to be problematic in gas formations. In this chapter, I develop a new formation parameter, $F_{p}$ using a combination of relevant petrophysical properties, such as neutron characteristic lengths and bulk density. In conjunction with FSFs and the linear iterative refinement (LIR) technique, the parameterization proved to be robust for rapid simulation of detector responses in any formation lithology, saturating fluid type, water salinity, fluid density, borehole size, well inclination, and mud filtrate invasion, with a 2-porosity unit (pu) accuracy. This tolerance is maintained for azimuthal measurements around the hole in presence of standoff up to 3 in. for varying stabilizer and borehole sizes - a seemingly reasonable tolerance in view of the borehole and environmental effects.

\section{$2.1 \quad$ INTRODUCTION}

Studies on the principles of neutron porosity measurement have been carried out for years and have provided a good understanding of petrophysical, geometrical and borehole environmental effects that contribute to measurement interpretation (Ellis et al., 2003; 2004; 2007). Development of several modeling methods for nuclear logging measurements has also improved understanding of factors that affect accurate petrophysical interpretation. Traditionally, borehole nuclear measurements are simulated with the Monte Carlo N-Particle (MCNP) program (X-5 Monte Carlo Team, 2003), where each particle is tracked in space and flux tally counts are averaged at detector locations. A faster method for modeling nuclear responses includes the use of differential sensitivity functions (Watson, 1984; Case et al., 1994; Mendoza et al., 2007; Zhou et al., 2009). This method has been elaborately applied and extended for interactive modeling 
(Heidari et al., 2009; Ijasan et al., 2011; 2013) and inversion (Sanchez et al., 2010; Heidari et al., 2012; Mendoza et al., 2012; Shetty et al., 2012) of wireline and loggingwhile-drilling (LWD) nuclear measurements. Using pre-calculated 3D spatial flux sensitivity functions (FSF) and the linear iterative refinement (LIR) technique (Mendoza et al., 2010a; 2010b), response changes that are due to borehole and formation perturbations are estimated according to first-order perturbation theory. This method significantly increases modeling speed while maintaining accuracy.

In the FSF method, the choice of an appropriate weighted parameter for perturbation estimates is crucial. The parameter is required to honor the approximations of first-order perturbation as formation properties, borehole conditions, and well deviation vary along the borehole, i.e., the parameter should be "well-behaved". In gamma-gamma measurements, bulk or electron densities are appropriately well-behaved parameters. In neutron logging measurements, the inverse of migration length (Ellis et al., 2007) is appropriate because of its direct dependence on macroscopic energy cross section. But in gas-saturated formations, the inverse of migration length is not wellbehaved because detector responses become nonlinear with respect to water-saturated formations. I observed the dependence of this nonlinearity on methane $\left(\mathrm{CH}_{4}\right)$ density in MCNP-calculated neutron detector counts of the EcoScope ${ }^{\dagger}$ multi-function LWD tool, shown in Figure 2.1. As saturating gas density decreases, the nonlinearity between detector responses and inverse of migration length is pronounced and the gas-saturated trend lines (red lines) deviate away from the water-saturated line (blue line). This suggests incorporating density in my formation parameter. This nonlinearity is further complicated by borehole geometrical and environmental effects.

\footnotetext{
$\dagger$ Mark of Schlumberger
} 
In this chapter, I developed a new formation parameter, $F_{p}$ that fits all detector responses, irrespective of formation fluid, type, salinity, or density, to a polynomial regression. This $F_{p}$ also suffices in predicting detector responses, using the FSF and LIR rapid simulation technique, in the presence of petrophysical and environmental perturbations in the borehole. As borehole size changes, $F_{p}$ perturbations appropriately respond to borehole size effects. This obviates the need to pre-calculate FSFs in different borehole sizes.

For benchmark purposes, FSF- $F_{p}$ fast-forward model (FFM) results are compared to MCNP calculations in terms of speed and accuracy for the EcoScope LWD neutron porosity measurement. I investigate standoff effects on azimuthal neutron porosity logs around the borehole for eccentered tool geometry in varying borehole sizes drilled with varying stabilizer sizes. Also, for the first time, the LIR technique is validated with azimuthal experimental data of the EcoScope tool acquired across formations of known properties. Using synthetic earth models, I demonstrate accurate and fast modeling of azimuthal neutron porosity measurements in high-angle/horizontal (HA/HZ) wells. The results shown in this chapter encourage development of petrophysical inversion methods, using the FSF- $F_{p}$ FFM, for LWD neutron porosity measurements in the presence of borehole and environmental effects.

\section{2 $F_{P}$ Formulation ANd PARAMETER ESTIMATION}

For each detector, the new formation parameter, $F_{p}$ is formulated thus:

$$
\begin{aligned}
& F_{p}=\frac{a}{\sqrt{L_{s}^{2}+\alpha L_{d}^{2}}}+b \rho_{b}, \\
& L_{d}=\sqrt{L_{m}^{2}-L_{s}^{2}}
\end{aligned}
$$


where $\rho_{b}$ is the formation volumetric bulk density in $\mathrm{g} / \mathrm{cm}^{3}, L_{d}$ is the formation diffusion length in $\mathrm{cm}, L_{s}$ is the slowing-down length in $\mathrm{cm}, L_{m}$ is the migration length in $\mathrm{cm}, \alpha$ is the diffusion parameter coefficient, $a$ is the neutron parameter coefficient, and $b$ is the density parameter coefficient; $\alpha$ and $a$ are unitless coefficients, while $b$ is in $\mathrm{cm}^{2} / \mathrm{g}$. Therefore, $F_{p}$ has a unit of $\mathrm{cm}^{-1}$, similar to the macroscopic energy cross section. Characteristic lengths $\left(L_{d}, L_{s}\right.$, and $\left.L_{m}\right)$ are calculated with SNUPAR (McKeon et al., 1989), the Schlumberger Nuclear Parameter calculator. Each detector's tally count responses, obtained from $\mathrm{MC}$ calculations, in the base case formations are fitted to a polynomial of degree $N$ as shown below, i.e., near and far detector counts are fitted separately,

$$
p=\log _{e}(\text { MCNP detector counts })=\sum_{n=0}^{N} m_{n} F_{p}^{n},
$$

where $m_{n}$ is $n$-th polynomial coefficient. To estimate the detector coefficients $(\alpha, a, b$ and $m_{n}$ ), a nonlinear parameter estimation problem is setup such that the least-square cost function to be minimized is

$$
e\left(\alpha, a, b, m_{n}\right)=\left\|p-\sum_{n=0}^{N} m_{n} F_{p}^{n}\right\|_{2}^{2} .
$$

Using Levenberg-Marquardt (LM) minimization method (Aster et al., 2005; Hansen, 1998), the inversion is posed such that 


$$
\begin{aligned}
& {\left[\begin{array}{l}
\mathbf{J}^{k} \\
\lambda^{k} \mathbf{I}
\end{array}\right] \Delta \mathbf{x}^{k}=\left[\begin{array}{c}
-\mathbf{e}^{k} \\
0
\end{array}\right],} \\
& \mathbf{x}^{k+1}=\mathbf{x}^{k}+\Delta \mathbf{x}^{k},
\end{aligned}
$$

where $\mathbf{J}$ is the Jacobian matrix, $\lambda$ is the stabilization parameter, $\mathbf{I}$ is an identity matrix, $\mathbf{e}$ is an misfit vector between MCNP detector counts and the polynomial regression, $\mathbf{x}$ is vector of detector coefficients, and superscript $k$ represents $k$-th iteration; $\Delta \mathbf{x}$ determines the direction towards convergence from the $(k-1)$-th iteration, and is given as

$$
\Delta \mathbf{x}=\left[\begin{array}{llll}
\Delta \alpha & \Delta a & \Delta b & \Delta \mathbf{m}_{n}
\end{array}\right]^{T} .
$$

The Jacobian matrix is calculated as

$$
\mathbf{J}=\left[\begin{array}{c}
\frac{\partial p}{\partial F_{p}} \frac{a L_{d}^{2}}{2\left(L_{s}^{2}+\alpha L_{d}^{2}\right)^{\frac{3}{2}}} \\
\frac{\partial p}{\partial F_{p}} \frac{1}{\sqrt{L_{s}^{2}+\alpha L_{d}^{2}}} \\
\frac{\partial p}{\partial F_{p}} \rho_{b} \\
\frac{\partial p}{\partial \mathbf{m}_{n}}
\end{array}\right]^{T},
$$

where

$$
\frac{\partial p}{\partial F_{p}}=\sum_{n=1}^{N} n m_{n} F_{p}^{n-1},
$$


and

$$
\frac{\partial p}{\partial m_{n}}=F_{p}^{n}
$$

Several strategies, including multi-start initial guesses (at $k=0$ ), were employed to ensure a well regularized, stable, and globally minimized solution from the LM inversion.

Using the EcoScope LWD tool, with a 7.875-in. housing diameter eccentered in a "slick" 8-in. freshwater-filled borehole, detector counts obtained from the limestone base cases (shown in Figure 2.1) are fitted to the polynomial function of equation 2.3 where $N$ is 2, i.e., a quadratic function. Limestone lithology is used as reference for neutron porosity units. The 8-in. freshwater-filled borehole situation is referred to as the standard condition. The regression is calculated separately for near and far detectors to account for the differing volumes of investigation (VOI), detector spacing, design, and shielding. Convergence is obtained, for each detector, after the least-square cost function in equation 2.4, from the $F_{p}$ regression remained at a constant flat minimum. Figure 2.2 shows the $F_{p}$ regression results. Table 2.1 shows the EcoScope $F_{p}$ parameter coefficients, after convergence, for each detector.

Since near and far detectors have separate sets of coefficients, $F_{p}$ s calculated for individual detectors are different. To measure the accuracy of the $F_{p}$ regression, I calibrated the final residuals, in equation 2.4, to neutron porosity, BPHI. BPHI is the EcoScope ratio-method compensated neutron porosity, in limestone units. Panel b of Figure 2.2 shows the BPHI residuals due to the regression. I observe that BPHI residuals are below 2 porosity units $(\mathrm{pu})$ for all base cases, irrespective of saturating fluid. It is worth noting that relative uncertainties from MCNP calculation of detector counts were maintained below $0.3 \%$. 
In Table 2.1, $m_{2}$ is quite small, suggesting an almost linear relationship between detector counts and $F_{p}$. As shown in panel a of Figure 2.2, this formulation is robust (unlike the inverse of migration length in Figure 2.1) over a wide range of gas densities, water salinities, and formation porosities.

\subsection{Petrophysical EfFects}

The $F_{p}$ formulation serves as a well-behaved weighted formation property for estimating detector changes due to petrophysical perturbations. I compare benchmark detector counts from MCNP to the FSF iterative refinement technique for rapid modeling of nuclear well logging measurements using $F_{p}$ perturbations. Under standard conditions (8-in. freshwater-filled borehole), porosity and gas crossover effects are adequately predicted by the FSF- $F_{p}$ method.

Figure 2.3 shows comparisons of MCNP and FSF- $F_{p}$ methods in limestone formations. For the purpose of petrophysical interpretation, porosity differences in panel c across gas-saturated formations are calculated with the Gaymard-Poupon approximation (Gaymard and Poupon, 1968), i.e.,

$$
\sqrt{\frac{D P H I^{2}+B P H I_{F S F}^{2}}{2}}-\sqrt{\frac{D P H I^{2}+B P H I_{M C N P}^{2}}{2}}
$$

where DPHI is density porosity obtained with a second-order gamma-gamma fastforward model (Zhou et al., 2009). Porosity differences across water-saturated formations are obtained by arithmetic difference of BPHI limestone units, i.e., $B P H I_{F S F}-B P H I_{M C N P}$. In panel a, I observe that the methods agree well as pore volume varies for water- and gas-saturated formations. In panel $\mathrm{b}$, qualitative comparisons of FSF- $F_{p}$ (green lines) to MCNP (black lines) indicate good agreement. Also, I observe an overlap in DPHI and 
BPHI, as expected, in water-saturated limestone formations (circular points) and a gas crossover effect, i.e., DPHI > BPHI (square points) in gas-saturated formations. Calculated porosity differences between MCNP and FSF- $F_{p}$ methods are within 2 pu, as shown in panel c. The source of these errors is primarily the $F_{p}$ regression in panel b of Figure 2.2.

In sandstone (Figure 2.4), under standard conditions, lithology effect is manifested as crossover between DPHI and BPHI in water-saturated formations (circular points in panel b), i.e., DPHI > BPHI. Also, note the good agreement between FSF- $F_{p}$ and MCNP calculations, green and black lines, respectively. In Figure 2.5, porosity logs in dolomite formations exhibit a reverse or matrix crossover in water-saturated dolomite, i.e., DPHI $<$ BPHI. Panel c error values are a combination of lithology effects and $F_{p}$ regression errors.

\subsection{Stabilizer AND Borehole Size EFFects}

In large hole sizes, perturbations from standard conditions in the borehole become significant. For example, in a limestone block, borehole properties change from 0 pu limestone in standard conditions to $100 \mathrm{pu}$ freshwater or mud, due to borehole evacuation. This creates a perturbation magnitude of $1.71 \mathrm{~g} / \mathrm{cm}^{3}$ and $16.44 \mathrm{~cm}$ in density and migration length, respectively. As discussed earlier, the density parameter is wellbehaved to honor first-order perturbation calculations for predicting gamma-gamma differential detector responses. Also for an eccentered tool, gamma responses are focused (azimuthal aperture is about $20^{\circ}$ ) at the bottom of the hole and azimuthal standoff effects are minimal. In neutron calculations, neutron responses have wide azimuthal apertures, about $120^{\circ}$ (Ijasan et al., 2011), such that azimuthal standoff significantly affects detector responses. The $16.44-\mathrm{cm}$ perturbation is quite significant considering the nonlinear 
behavior of neutron responses over this range. These effects create significant errors when the inverse of migration length is used as the weighted parameter.

One brute-force solution is to generate FSFs in the borehole sizes under investigation, i.e., sizes other than standard condition. With this approach, the perturbation in the borehole is constrained to mud property changes only, such as mud weight, mud salinity, and/or mud composition. Perturbations due to mud salinities are usually below $1 \mathrm{~cm}$ in migration length, and hence are covered under first-order approximations. The main disadvantage of this brute-force approach is that the FSF library increases in size and consumes disk space. Also, arbitrary hole sizes and shapes due to rugosity cannot be modeled accurately. Chapter 3 discusses a more dynamic approach which invokes diffusion models to characterize 3D flux differences or perturbations and account for higher-order nonlinear transport effects (Ijasan et al., 2013).

Figure 2.6 shows the schematic of tool-borehole geometry for an eccentered configuration. With varying stabilizer sizes, minimum standoff at the bottom and maximum standoff at the top of the hole can be varied to investigate FSF- $F_{p}$ sensitivity to borehole standoff. For Monte Carlo (MC) benchmark purposes, the standard borehole size is 8 in. (no standoff), while other stabilizer diameter sizes include 8.25 in. and 9.375 in. Borehole sizes are varied between 8 in. and 12 in. Homogeneous base cases consisting $0-40 \%$ pore volume water- and gas-saturated limestone formations are used for the benchmark. All boreholes are filled with freshwater.

I compared detector responses predicted using $F_{p}$ perturbation estimates in the FSF technique (standard conditions) with MCNP results. Figures 2.7 and 2.8 show LWD detector responses in bottom and top tool orientations, respectively, around a 10-in. borehole drilled with an 8.25-in. stabilizer. Calculated porosity differences between MCNP and FSF- $F_{p}$ are shown in panels $\mathrm{c}$ of Figures 2.7 and 2.8. It is worth noting that 25 
magnitudes of porosity difference, for $0-40 \%$ pore volume of water- and gas-saturated limestone formations shown in Figures 2.7 and 2.8, are below 2 pu. Top sector responses (Figure 2.8) are also within the $2 \mathrm{pu}$ error limit despite having the most pronounced borehole effects in the 10-in. borehole. This is good indication of FSF- $F_{p}$ technique's robustness.

\subsection{StandofF SENSITIVITy Analysis}

With a housing diameter of 7.875-in., stabilizer diameter sizes of 8.25-in. and 9.375-in. are used to investigate standoff effects as the tool rotates around the hole (Figure 2.6) in borehole sizes varying from 8 in. to 12 in. Radial standoff thicknesses directly in front of tool housing are calculated for each eccentered tool orientation, i.e., top, right, bottom, and left. Due to symmetry, radial standoff at right and left tool orientations are equal.

Figure 2.9 shows calculated porosity differences between MCNP and FSF- $F_{p}$ in several borehole sizes for the formation base cases considered, i.e., 0 - 40\% pore volume, water- and gas-saturated limestone. The legend labels designate the formation saturating fluid, stabilizer size, and borehole size, e.g., g0825_1000 represents an 8.25-in. stabilizer across a gas-saturated limestone formation in a 10-in. borehole. As the eccentered tool rotates around the borehole, the detectors view azimuthally dependent radial standoff around the borehole. The radial standoff size is calculated from geometry and plotted on the $\mathrm{x}$-axis while porosity differences are plotted on the $\mathrm{y}$-axis for the borehole sizes and tool orientations.

In borehole sizes less than 10 in., panels a and b of Figure 2.9, porosity differences are within $\pm 2 \mathrm{pu}$. In the 12-in. borehole, panel c of Figure 2.9, as standoff in front of the detector increases above 1.5 in., porosity difference exceeds 2 pu. On the 
other hand, porosity differences for standoff greater than 1.5 in. in 10-in. borehole, panel b, are within \pm 2 pu. This discrepancy between standoff sensitivity in 10-in. and 12-in. boreholes is attributed to neutron VOI effect. In a 12-in. borehole, for the stabilizer sizes shown, neutron VOI encompasses more borehole fluid than in a 10-in. borehole, for the same radial standoff thickness. This results in a more pronounced borehole environmental effect, a strain in the FSF- $F_{p}$ prediction of detector responses, and thus larger porosity differences. At larger radial standoff sizes above 3 in., porosity differences increase to about $12 \mathrm{pu}$, panel $\mathrm{c}$ of Figure 2.9. At these standoff ranges, the detectors respond primarily to borehole fluid and measurements suffer significant borehole effect.

\subsection{INVASION EFFECTS}

To investigate invasion effects, I model freshwater invasion of a 30-pu gassaturated limestone formation. I performed MCNP and FSF- $F_{p}$ simulations for several piston-like invasions, with radial depths ranging from the borehole wall to 20 in. into the formation. Also, I assumed zero residual gas saturation. This implies that the flushed zone consists of 30-pu water-saturated limestone, and there is no transition zone. Figure 2.10 shows comparison of MCNP and FSF- $F_{p}$ methods for the invasion case. From the detector counts in panels a and b, I observed good agreement between MCNP and FSF- $F_{p}$ calculations. It is worth noting that detector counts asymptote to $90 \%$ of the flushed zone response at $7.12 \mathrm{in.}$ and $4.26 \mathrm{in.}$ for the far and near detectors, respectively. This indicates different detector depths of investigation (DOI). In panel c, I observed gas crossover (yellow shaded region) between DPHI and BPHI logs when the invasion front is less than 9 in. from the borehole wall. Note that DPHI asymptotes to $90 \%$ of the flushed zone response at a radial depth of $3.88 \mathrm{in}$. 
This indicates much shallower density DOI than neutron. This implies that in the region between 3.88 in. and 9 in. (hatched region), density and neutron measurements respond to different gas saturations $\left(S_{g}\right)$, i.e., $S_{g}=0$ and $S_{g}>0$, respectively. Hence combined interpretation from neutron and density measurements in this region is unreliable. Panel d describes BPHI difference between MCNP and FSF- $F_{p}$ methods. Apart from $F_{p}$ regression errors, other sources of errors are due to the DOI and $S_{g}$ discrepancies among neutron near detector, neutron far detector, and density response (Ijasan et al., 2011). Notwithstanding, BPHI error differences are within an acceptable range of about 2 pu for FSF- $F_{p}$ method.

\subsection{EXPERIMENTAL VALIDATION}

The FSF- $F_{p}$ FFM, with LIR, is validated with experimental neutron porosity data acquired with the EcoScope tool in top, right, left, and bottom orientations, across watersaturated limestone formations of $0.5,16$, and $42.3 \%$ porosities in a 10 -in. borehole. The borehole is filled with freshwater. Figure 2.11 shows the comparisons between experimental or environmental effects characterization (EEC) data and neutron FFM. The black error bars in panel c of Figure 2.11 represent the \pm 2 pu error ranges from the benchmark results. I observe that most of the data points lie within \pm 2 pu error range.

Geometrically, the left and right tool orientations consist of equal radial standoffs, thus resulting in an azimuthally symmetrical LWD response. But EEC and FFM results in Figure 2.11 exhibit some degree of asymmetry. At this point, it is worth noting that the EcoScope far response consists of two adjacent thermal detectors, while the near response consists of a single thermal detector from a pair of adjacent detectors, i.e., thermal and epithermal. Thus the detector VOI responses are at slightly different azimuthal angles, such that asymmetry is observed between the dual detector responses. 
Their responses are such that the near detector lags behind the far detector in azimuth around the hole by about $10^{\circ}$. This is the source of asymmetry observed in Figure 2.11. This behavior is also observed in MCNP calculations. It is quite exceptional that this effect, as evident in experimental and MC results, is reproduced by the FFM, thus increasing my confidence in the FSF- $F_{p}$ LIR technique. It is worth noting that this is the first time LIR technique is being validated with experimental data.

\subsection{Modeling LWD Neutron Porosity Measurements in High- ANGLE/Horizontal WeLlS}

In $\mathrm{HA} / \mathrm{HZ}$ wells, the relative bed orientation plays a very important role in detector responses, especially at bed boundaries. Due to the radial DOI of the detectors, depth-shifts are observed at bed-boundaries because the tool senses the bed before arriving at the boundary's intersection with the borehole. Azimuthal corrective depthshifts are necessary, in multi-sector measurements, to eliminate detector mismatch horns due to bed dip (Guo et al., 2008). In dipping thin beds, density and neutron detectors sense multiple layers differently due to their different VOIs. This effect is pronounced at the bed boundaries such that I observe false gas crossovers. Using two synthetic earth models, the FSF- $F_{p}$ LIR simulation technique is applied to borehole azimuthal neutron porosity modeling in $\mathrm{HA} / \mathrm{HZ}$ wells. To obtain borehole neutron count rates and porosity images, a 16-sector azimuthal binning scheme is used for tool rotation measurements. The first synthetic model considered consists of 2.5 -ft layer formations. The formations have 10-pu water-saturated limestone layer sandwiched between 30-pu gas-saturated limestone layers. A sampling rate of $4 \mathrm{~cm}$ in true vertical depth (TVD) is used to log the formations in an $89^{\circ}$ well. Azimuthal MCNP and FSF simulation results are shown in Figure 2.12. Note that azimuthal corrective depth-shifts (Yin et al., 2008) have been 
applied in post-processing of detector responses, in both MCNP and FFM results. Panel i shows the differences between MCNP and FSF azimuthal neutron porosity logs.

I observe that all residuals are within $\pm 0.5 \mathrm{pu}$, irrespective of azimuthal angle, indicating good agreement with MCNP results. A unique feature observed in this synthetic model, as the tool transverse into the middle layer, is that the far detector counts decrease, panel a, while the near detector counts increase, panel c. This is an unusual response characteristic which the FSF- $F_{p}$ method reproduces with ease, panels $\mathrm{b}$ and $\mathrm{d}$, thus emphasizing the strength of the FSF- $F_{p}$ simulation technique. Panel g shows the compensated density image obtained from a second-order density model. As expected, I observed gas crossover in the gas layers and an overlap in the water-saturated layer of the bottom quadrant logs in panel h. The observed variations in the near detector response across the middle layer in panel $\mathrm{c}$ are due to MCNP uncertainties.

The second synthetic example consists of a 0.5 -ft 4-pu water-saturated limestone layer sandwiched between 30-pu water-saturated limestone formations. A sampling rate of $1 \mathrm{~cm}$ in true vertical depth (TVD) is used in an $85^{\circ}$ well. Results are plotted in Figure 2.13. Panel $h$ shows bottom quadrant BPHI and DPHI porosity logs. The neutron measurement has a larger VOI than the density measurement. Hence, as the tool transitions from a high porosity zone to a low porosity zone, the neutron measures lower porosity than the density, i.e., it responds to the lower porosity zone more than the density measurement. This differing VOIs between density and neutron logs results in the characteristic false gas cross-overs, particularly pronounced at bed boundaries. Porosity differences, within the layers, between MCNP and FSF- $F_{p}$ methods, shown in panel i, are within $\pm 2 \mathrm{pu}$. 


\subsection{SUMMARY AND DISCUSSION}

The reason for numerical simulation studies of nuclear measurements is to improve understanding of how measurement physics affects petrophysical interpretation, and thus incorporate these insights into formation evaluation. Current applications of FSFs and LIR for joint inversion (Heidari et al., 2012; Mendoza et al., 2012) have shown to enhance petrophysical interpretation by considering invasion properties, shoulder bed effects, and relative bed deviation.

The new $F_{p}$ is a characteristic of the formation, derived from neutron and density parameters, directly related to the macroscopic cross section for characterizing FSF perturbations. It also represents a measure of flux attenuation factor, i.e., $e^{F_{p}}$, for a given formation base case. Note that $F_{p}$ is negative (Figure 2.2). The ability of FSF- $F_{p}$ FFM to replicate characteristic response behaviors such as false gas crossover, response asymmetry, VOI effects, bed geometry effects, etc., encourages its applications for improved modeling-based petrophysical interpretation and development of inversion techniques.

The discrepancies in VOIs between neutron and density measurements introduce false gas crossovers at bed boundaries and across thin beds. This effect is pronounced when there is a significant contrast between the bed layers. In the presence of invasion, integrated interpretation using density and neutron measurements could be misleading because of inherent inaccuracies due to differing VOIs. Similarly, volume of borehole standoff is more representative of environmental effects than radial standoff.

For the HA/HZ cases described in this chapter, MCNP required 4.8 hours (17280 seconds) of CPU time per sample point on a Linux cluster; while LIR required 0.47 seconds per sample point on a Windows XP dual-core system using MATLAB. This 
tremendous improvement in simulation speed presents LIR as an efficient method for petrophysical inversion where accuracy of the $F_{p}$ formulation can be investigated further.

\subsection{Conclusions}

I have described fast-forward modeling of neutron porosity measurements in the presence of borehole and environmental effects using the FSF and LIR techniques, in conjunction with a new neutron-density formation parameter, $F_{p}$. The new $F_{p}$ obtained from nonlinear regression of detector counts to a neutron-density parameterized model, fits all detector responses in limestone formations saturated with various fluid constituents and properties, including saltwater of various salinities and methane gas of various densities. This parameterization solves the unconformable behavior of gas formations when fitting with characteristic lengths or their inverses while maintaining regression errors below 2 pu.

Using the FSF rapid simulation method with $F_{p}$ perturbations, porosity, gas, lithology, and borehole size effects are accurately estimated to within 2 pu errors when benchmarked to MCNP. Also, detector responses in the presence of invasion and well

deviation are accurately predicted with the FSF- $F_{p}$ method. Hence the $F_{p}$ formulation is robust and well-behaved for neutron measurements. It improves the FSF technique in comparison to the use of migration length perturbations. The FSF- $F_{p}$ FFM technique adequately represents the measurement physics of neutron porosity logging, while drastically improving modeling speed.

Petrophysical inversion requires fast-forward methods for estimating tool responses. The developments in this chapter encourage joint inversion of neutron porosity measurements with other well logs, in the presence of borehole and environmental effects. 
Table 2.1: $\quad F_{p}$ formulation coefficients, for limestone porosity units.

\begin{tabular}{|c|c|c|}
\hline \multicolumn{3}{|c|}{ EcoScope $\boldsymbol{F}_{\boldsymbol{p}}$ coefficients } \\
\hline $\mathbf{x}$ & Near detector & Far detector \\
\hline$\alpha$ & 0.5465 & 1.523 \\
\hline$a$ & -200.01 & -250.01 \\
\hline$b\left(\mathrm{~cm}^{2} / \mathrm{g}\right)$ & 0.9472 & 4.0619 \\
\hline$m_{0}$ & -12.97 & -12.834 \\
\hline$m_{1}$ & 0.515 & 0.0825 \\
\hline$m_{2}$ & 0.0092 & 0.000586 \\
\hline
\end{tabular}




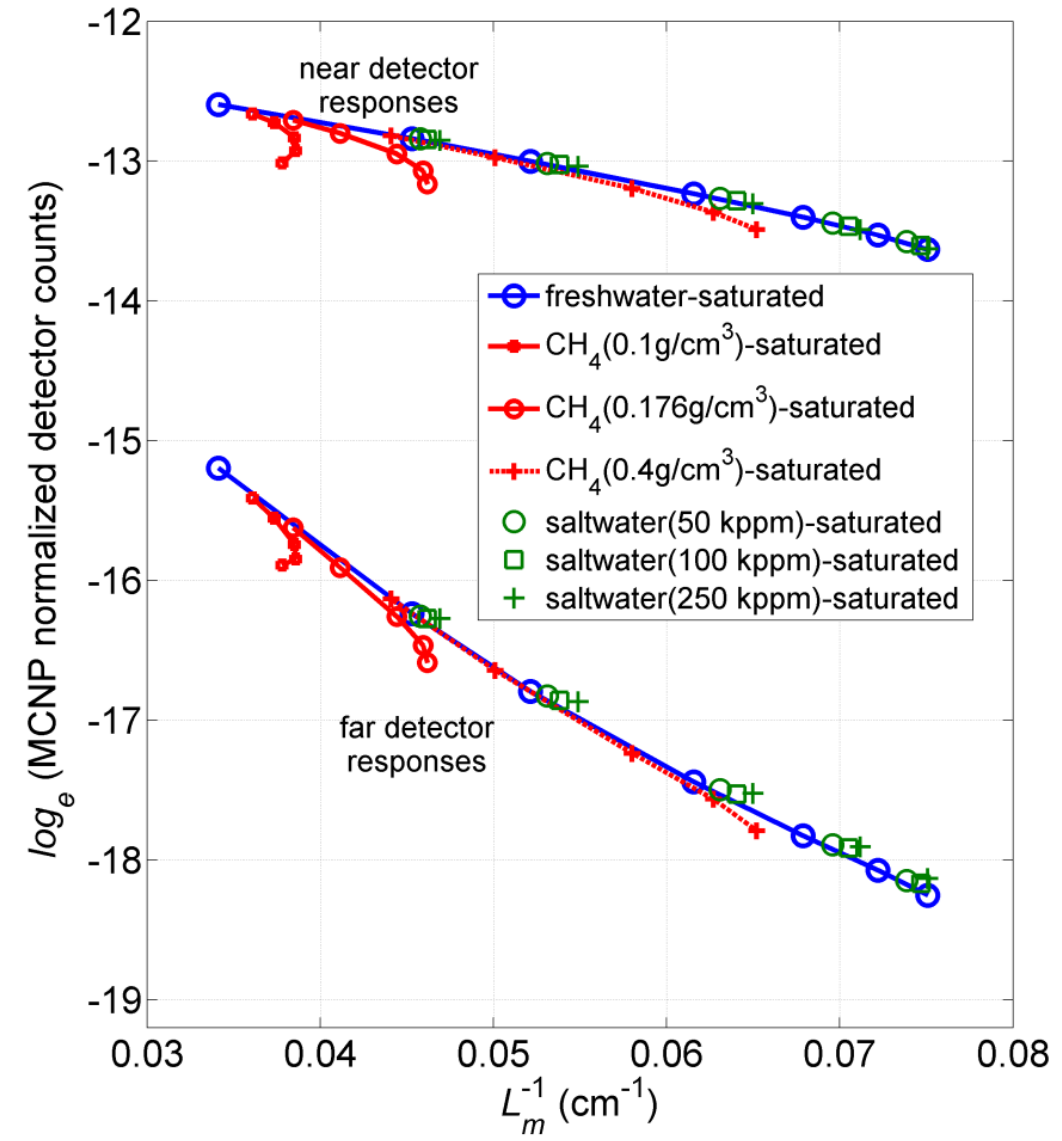

Figure 2.1: Near and far detector counts, in various porosities of limestone freshwater-, gas-, and saltwater-saturated formations, versus inverse of formation migration length. 
(a)

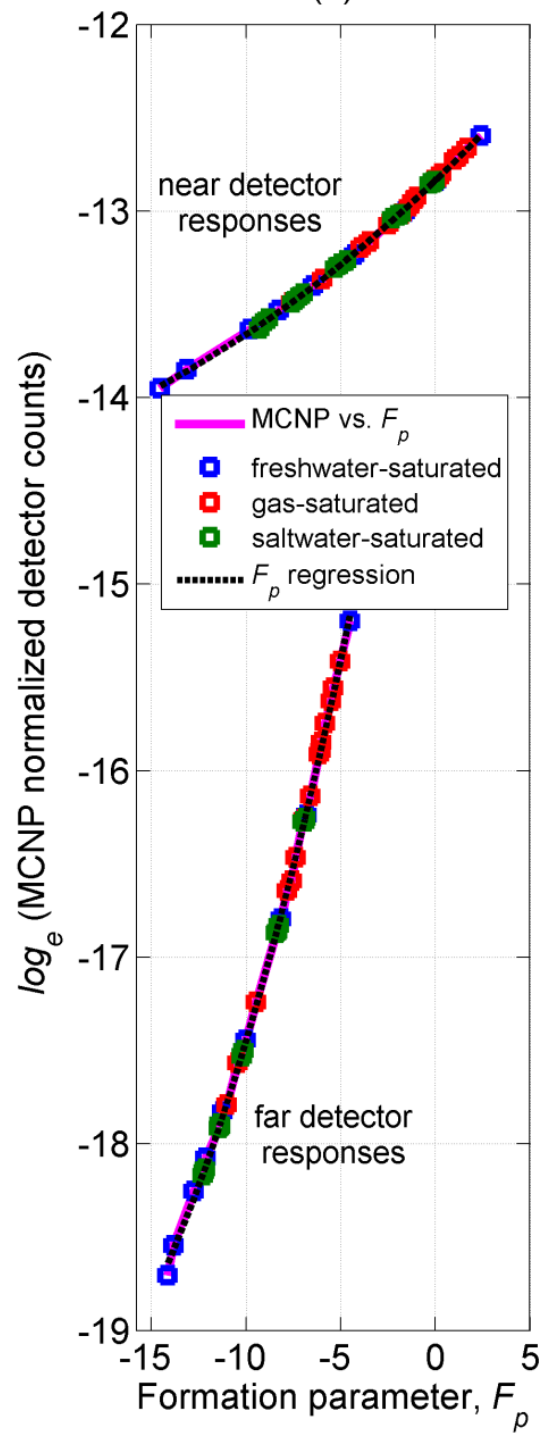

(b)

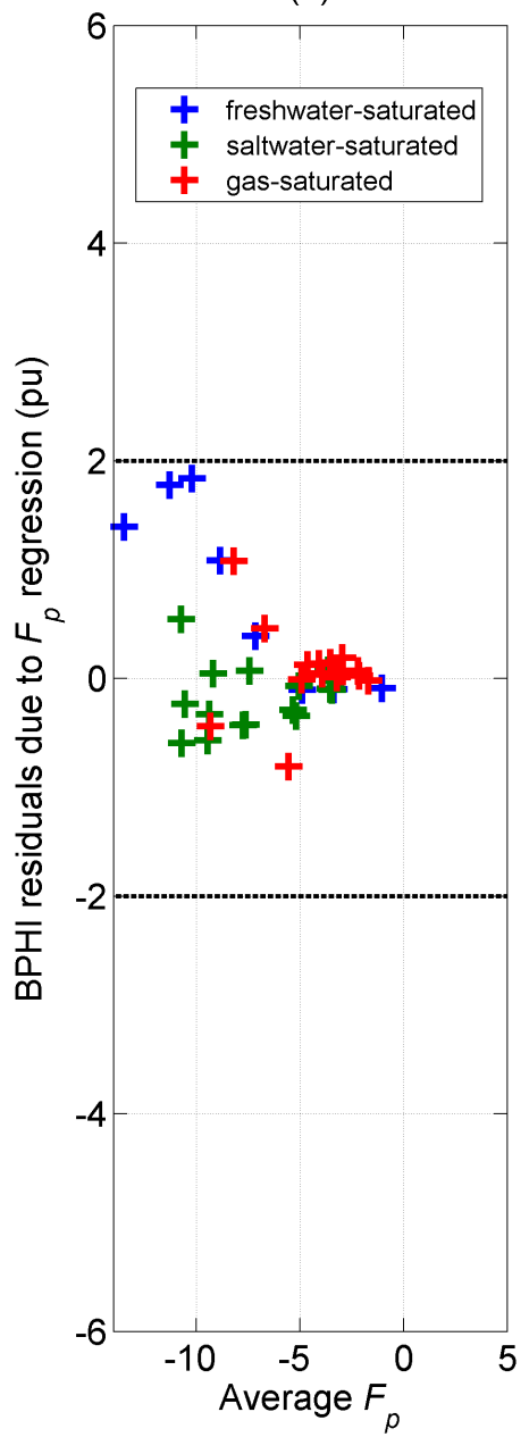

Figure 2.2: (a) Regression of far and near detector counts, across base cases of various fluid properties, with $F_{p}$ parameterization. (b) Computed BPHI (neutron porosity) differences, due to residuals in panel a, versus the average of near and far detector $F_{p}$. 
(a)

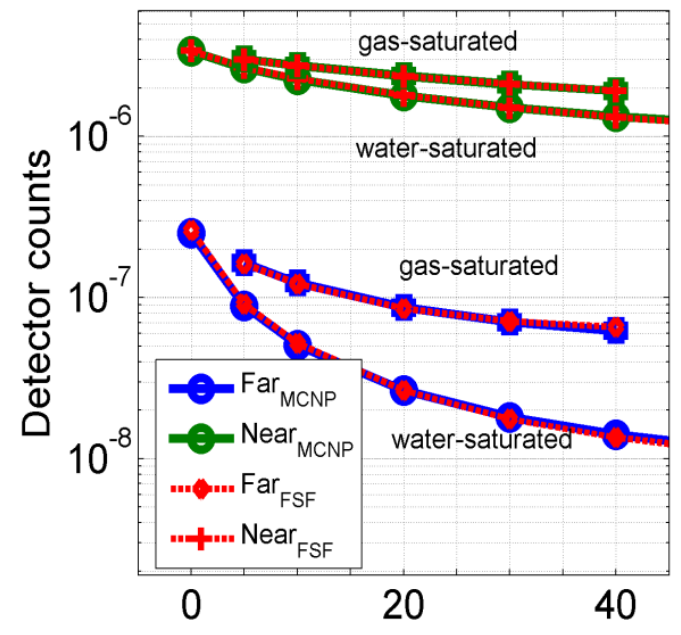

(b)

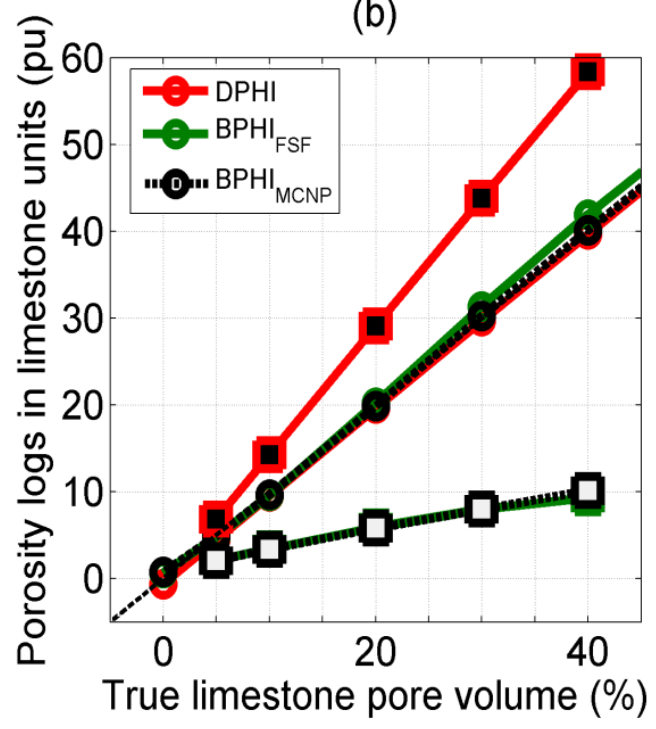

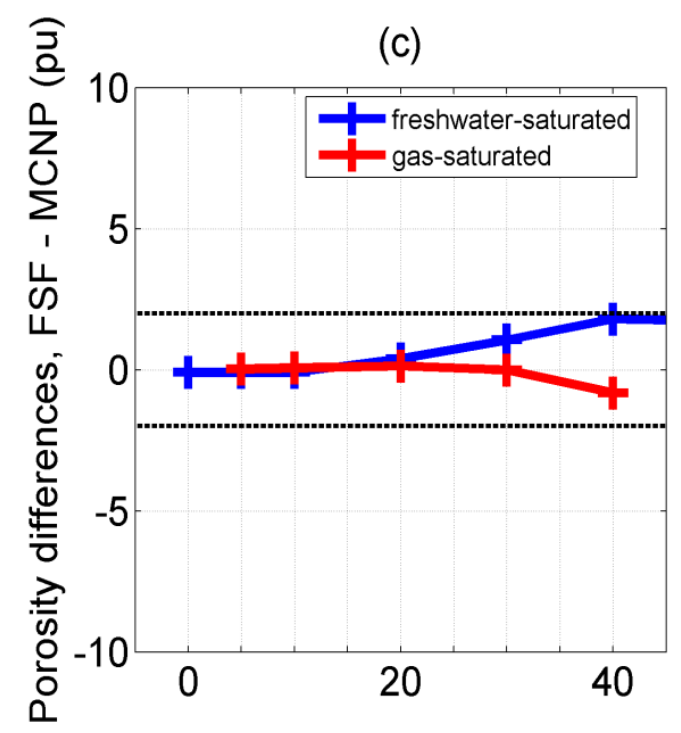

Figure 2.3: $\quad$ Benchmark results in slick 8-in. borehole. (a) Detector counts obtained from MCNP and FSF- $F_{p}$ FFM for freshwater- and gas-saturated limestone formations versus true pore volume in \%. (b) Density (DPHI) and neutron (BPHI) porosity logs versus true pore volume. Circular and square points represent water- and gassaturated formations, respectively. (c) Calculated porosity differences between MC and FSF- $F_{p}$ methods. 

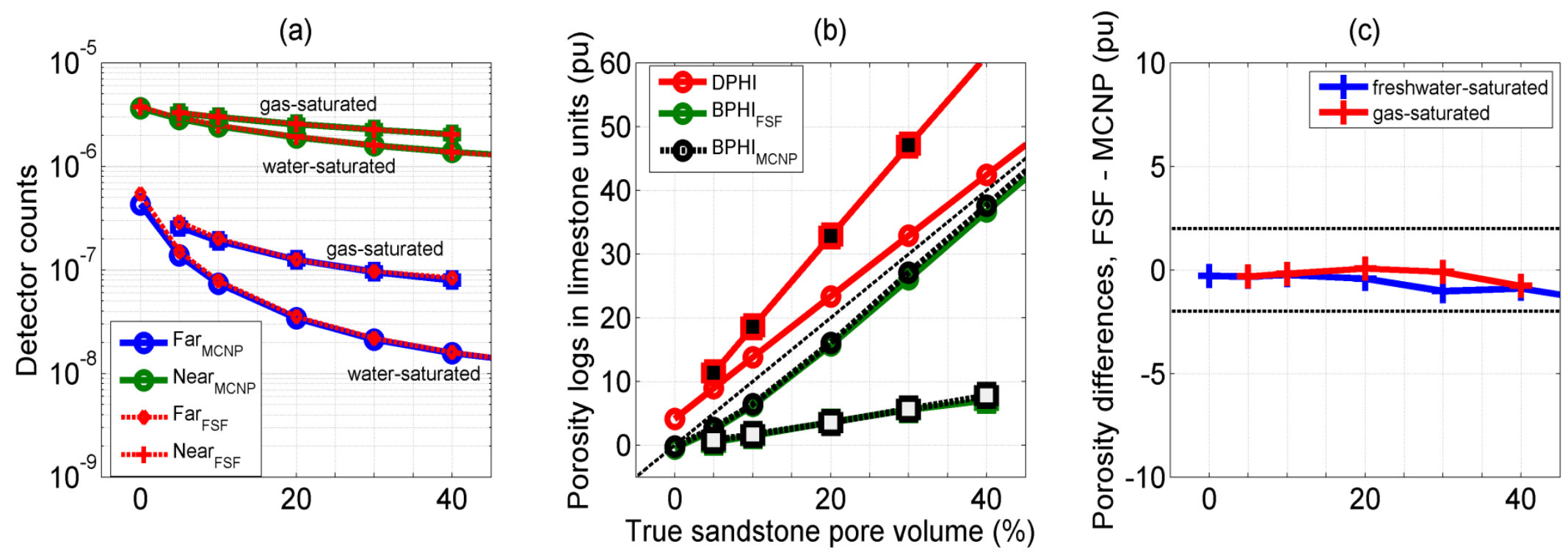

Figure 2.4: $\quad$ Benchmark results in slick 8-in. borehole. (a) Detector counts obtained from MCNP and FSF- $F_{p}$ FFM for freshwater- and gas-saturated sandstone formations versus true pore volume in \%. (b) Density (DPHI) and neutron (BPHI) porosity logs versus true pore volume. Circular and square points represent water- and gassaturated formations, respectively. (c) Calculated porosity differences between MC and FSF- $F_{p}$ methods. 

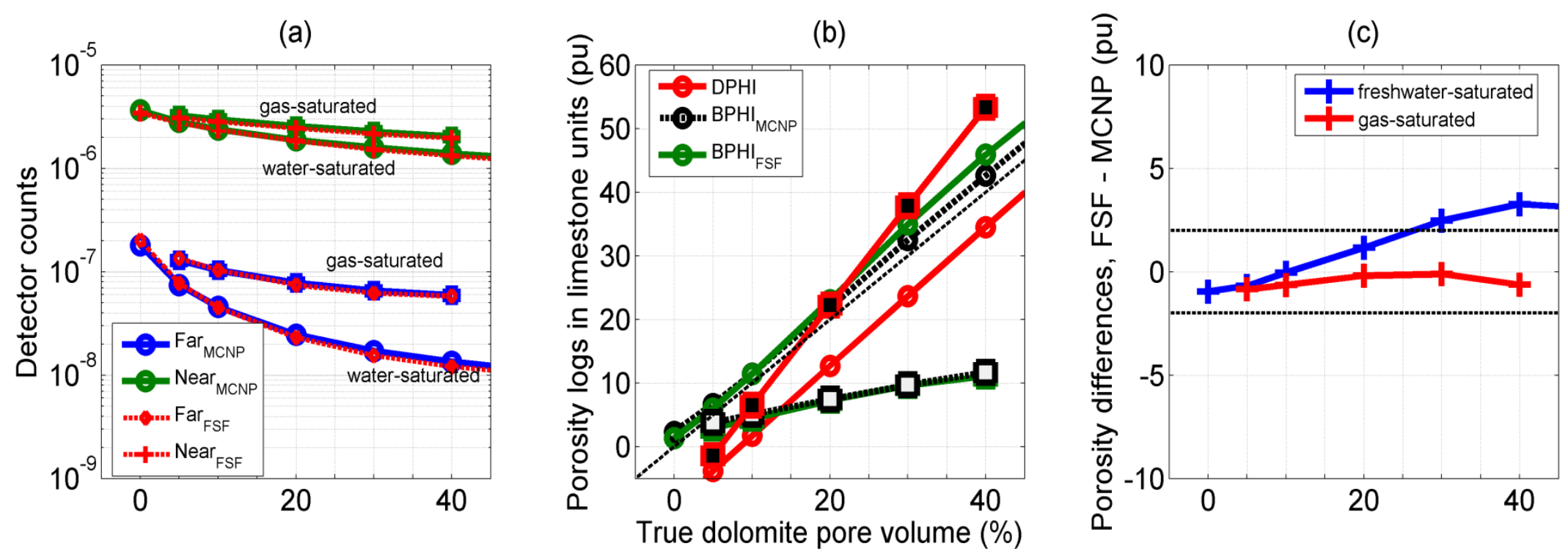

Figure 2.5: $\quad$ Benchmark results in slick 8-in. borehole. (a) Detector counts obtained from MCNP and FSF- $F_{p}$ FFM for freshwater- and gas-saturated dolomite formations versus true pore volume in \%. (b) Density (DPHI) and neutron (BPHI) porosity logs versus true pore volume. Circular and square points represent water- and gassaturated formations, respectively. (c) Calculated porosity differences between MC and FSF- $F_{p}$ methods. 
Schematic of LWD borehole and tool assembly
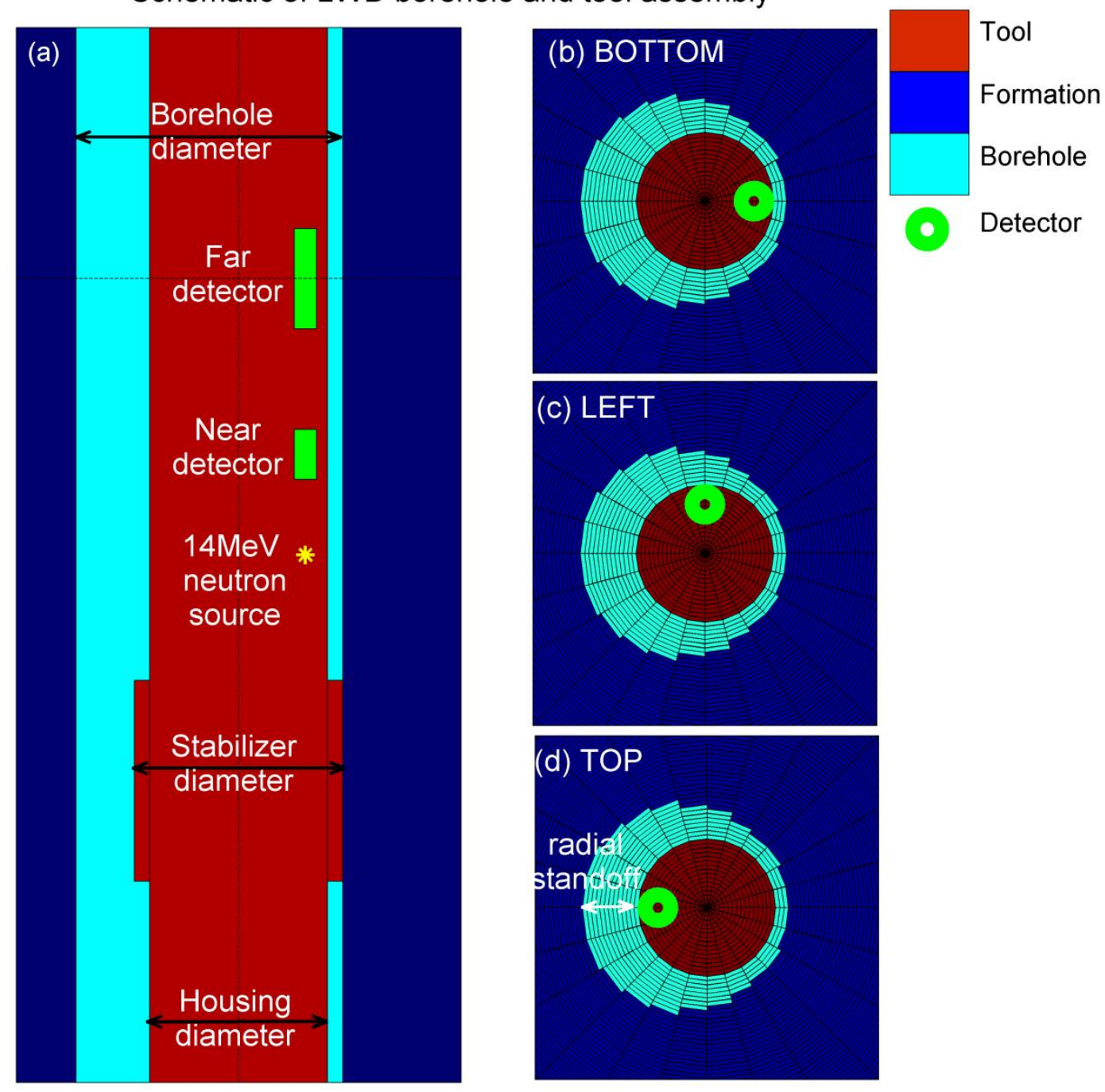

Figure 2.6: Schematic diagram showing LWD tool-borehole geometry. (a) Vertical cross-section showing tool housing, stabilizer, and borehole. Horizontal cross-section showing tool at (b) bottom, (c) left, (d) top orientation in the hole. 
Tool orientation: BOTTOM with 0.188 " radial standoff
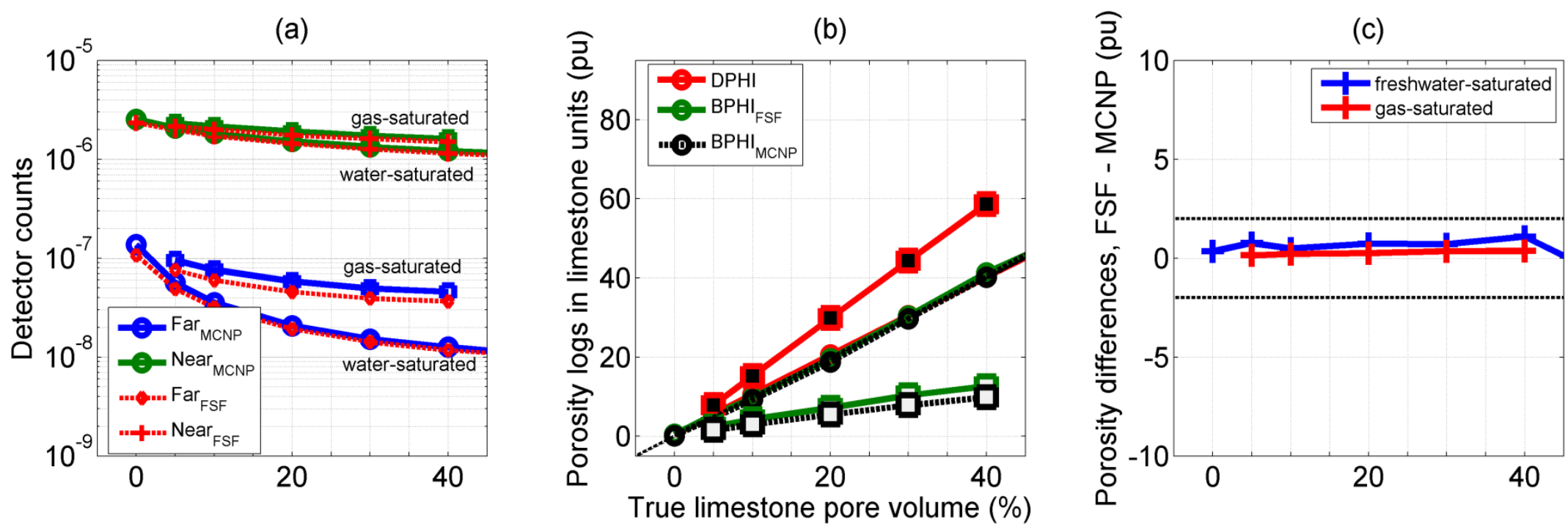

Figure 2.7: Benchmark results of an 8.25-in. stabilizer at the bottom of a 10-in. borehole. (a) Detector counts obtained from MCNP and FSF- $F_{p}$ FFM for freshwater- and gas-saturated limestone formations versus true pore volume in $\%$. (b) Density (DPHI) and neutron (BPHI) porosity logs versus true pore volume. Circular and square points represent water- and gas- saturated formations, respectively. (c) Calculated porosity differences between MC and FSF- $F_{p}$ methods. 
Tool orientation: TOP with 1.938" radial standoff
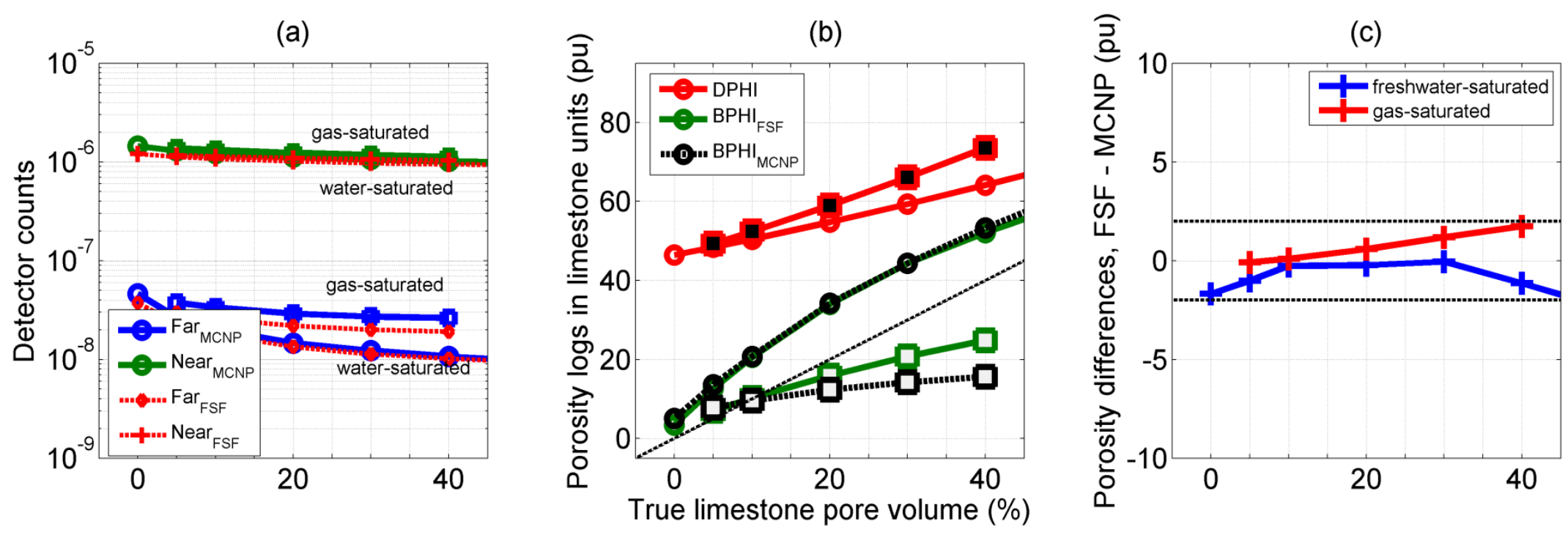

Figure 2.8: Benchmark results of an 8.25-in. stabilizer at the top of a 10-in. borehole. (a) Detector counts obtained from MCNP and FSF- $F_{p}$ FFM for freshwater- and gas-saturated limestone formations versus true pore volume in $\%$. (b) Density (DPHI) and neutron (BPHI) porosity logs versus true pore volume. Circular and square points represent water- and gas- saturated formations, respectively. (c) Calculated porosity differences between MC and FSF- $F_{p}$ methods. 
FSF-Fp FFM standoff sensitivity analysis across homogeneous limestone formations, water- and gas-saturated $0-40 \%$ pore volume

(a)

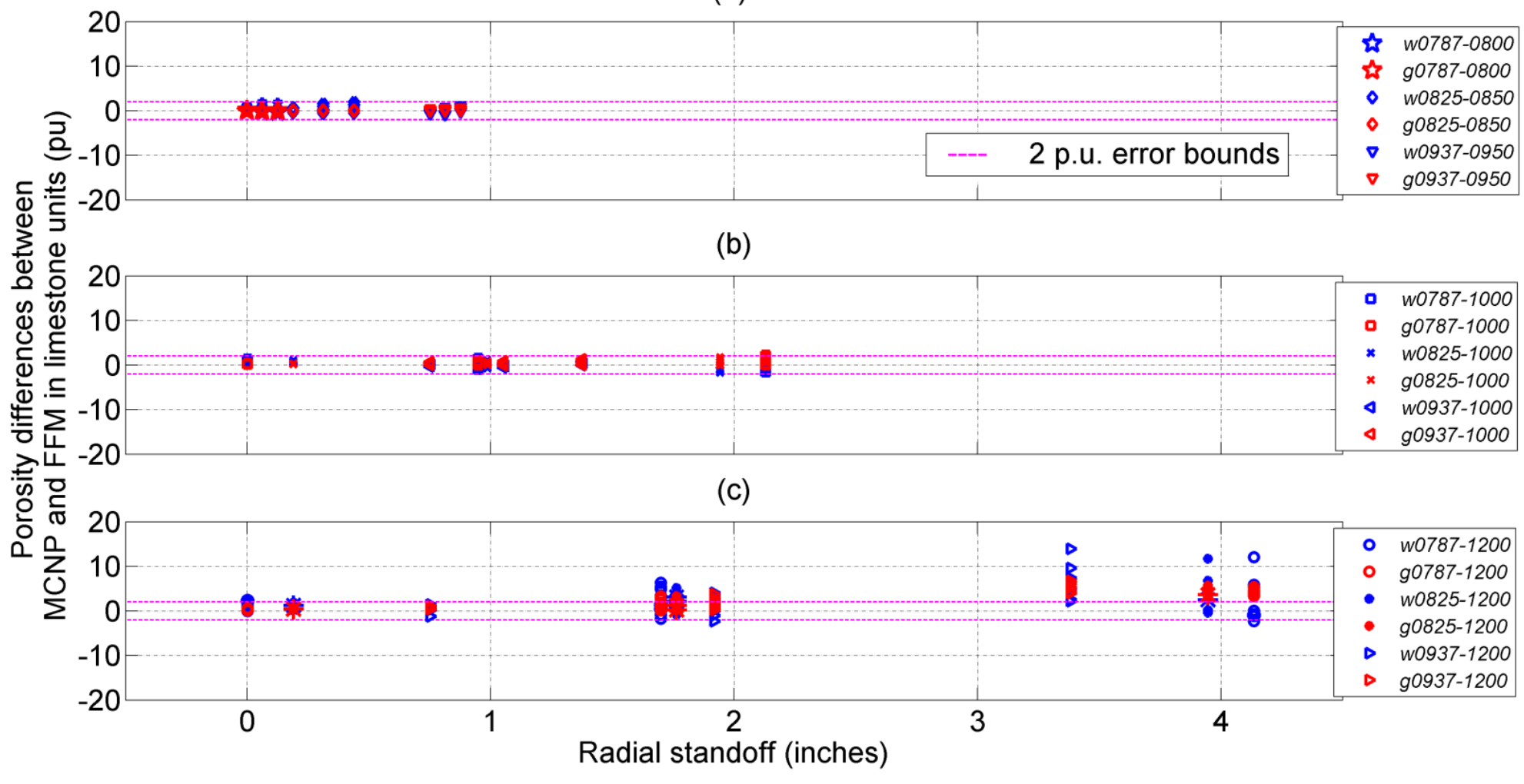

Figure 2.9: LWD standoff sensitivity analysis of FSF- $F_{p}$ FFM, in comparison to MCNP, around the borehole using 7.875 in., 8.25 in., and 9.375 in. stabilizer sizes. Calculated porosity differences in (a) 8-in., 8.5-in., and 9.5-in. borehole sizes, (b) 10-in. borehole size, and (c) 12-in. borehole size. 

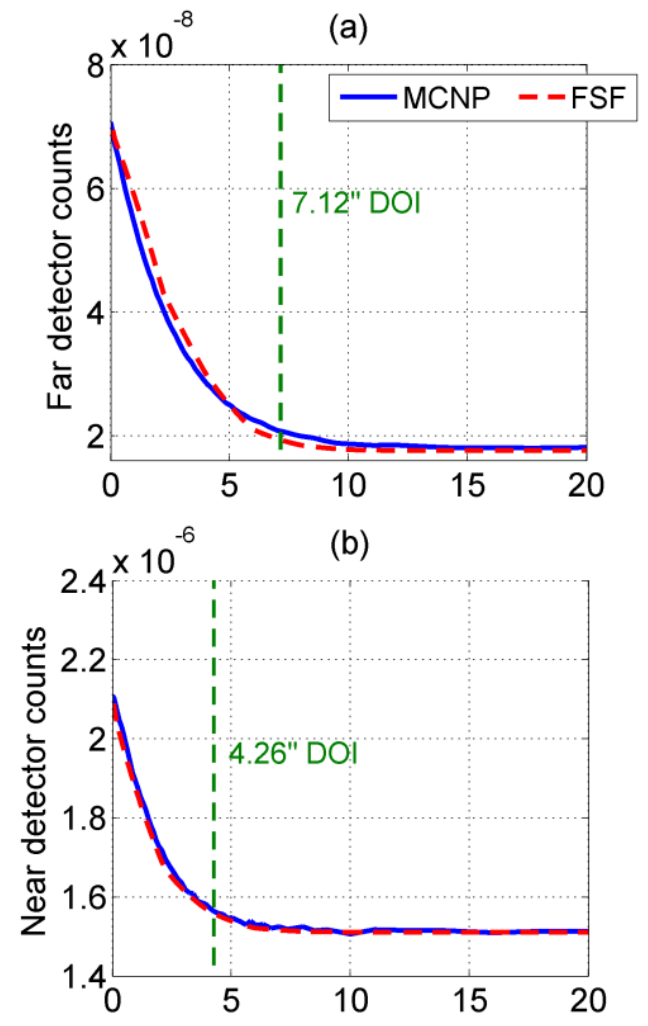

(c)

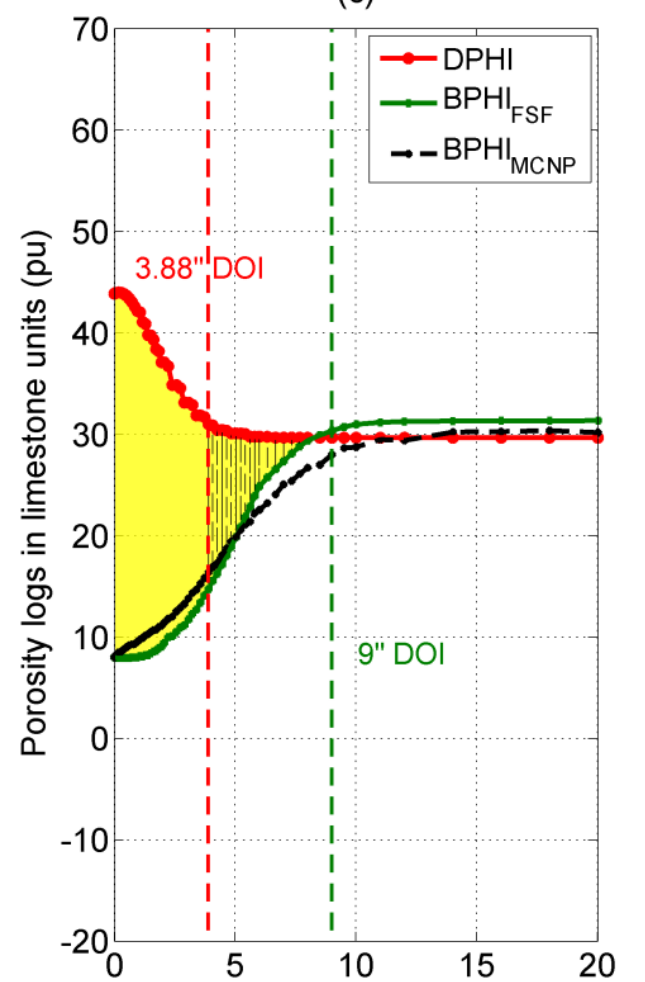

(d)

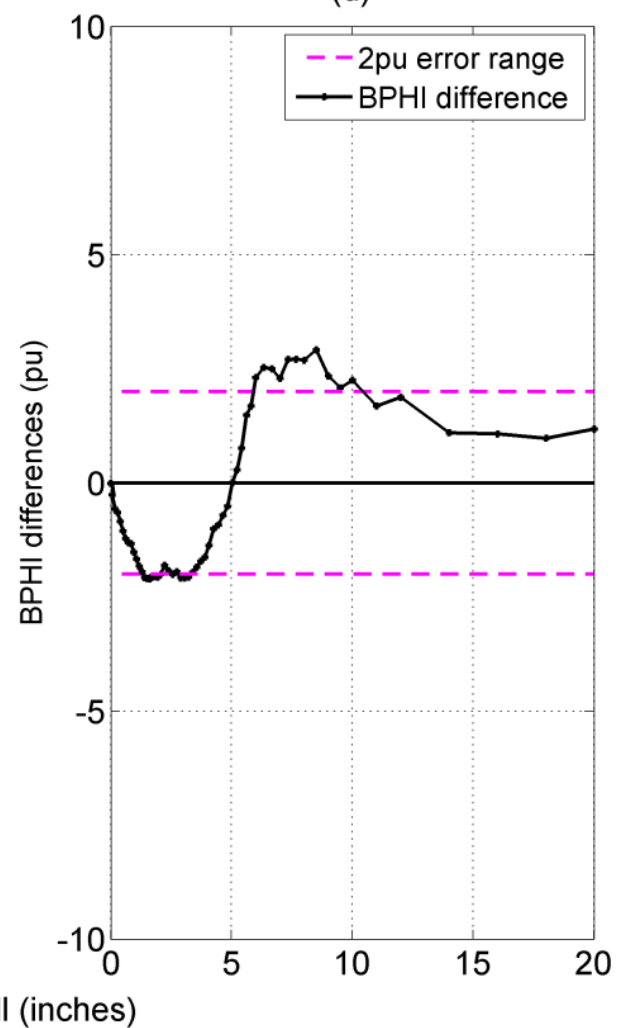

Figure 2.10: Detector counts for $\mathrm{MC}$ and FSF- $F_{p}$ methods versus radial distance of invasion front from the borehole wall for (a) far detector with 7.12 in. DOI, and (b) near detector with 4.26 in. DOI. (c) DPHI and BPHI porosity logs versus radial distance of invasion front. $\mathrm{MC}$ and FSF- $F_{p}$ BPHIs in black and green curves, respectively. Red vertical line represents 3.88 in. density DOI, while green vertical line represents 9 in. neutron porosity DOI. (d) BPHI differences between MC and FSF- $F_{p}$ methods. 

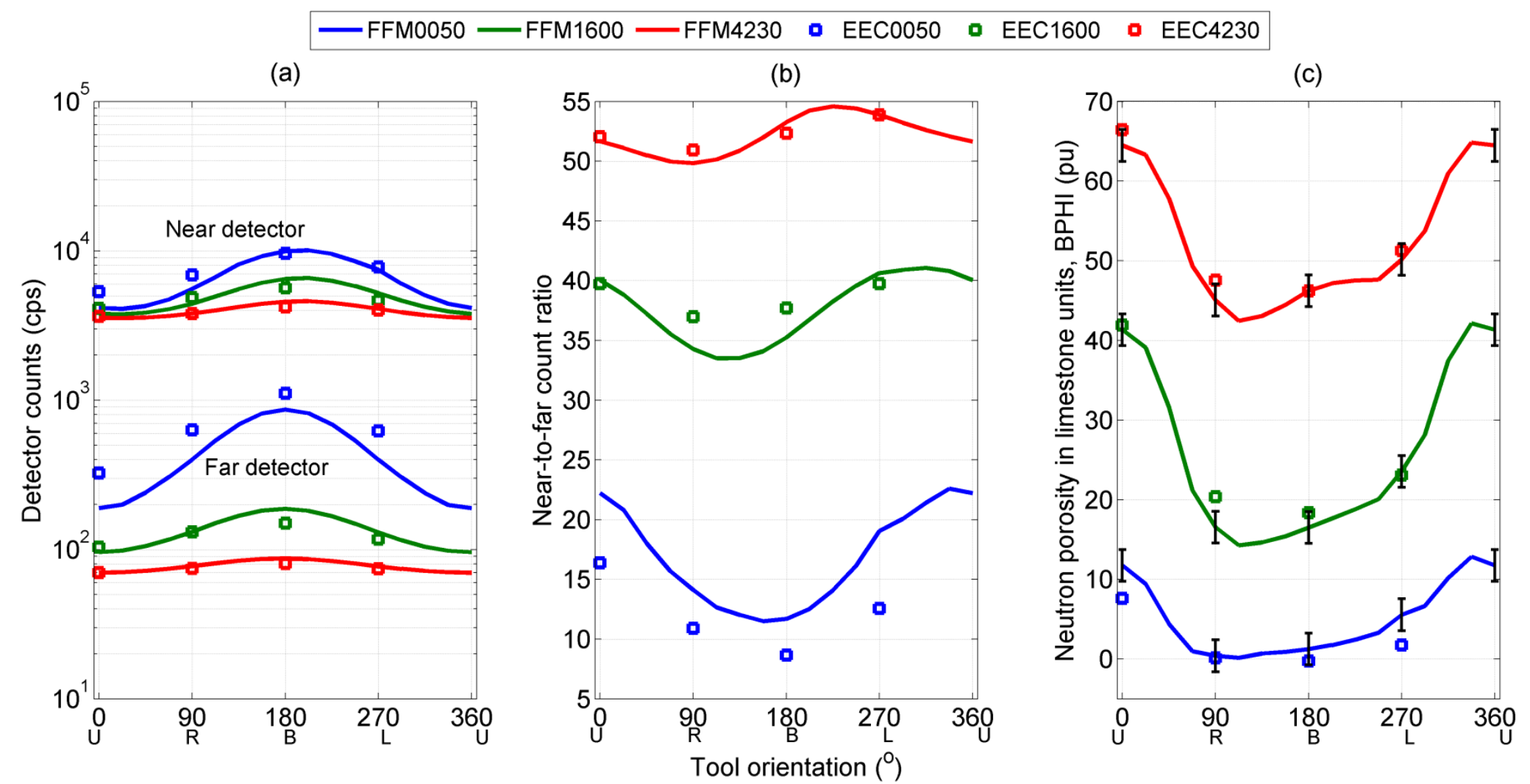

Figure 2.11: Experimental validation of FSF- $F_{p}$ FFM, with LIR, in a 10-inch borehole. (a) Far and near detector counts per second. (b) Near-to-far count ratios. (c) BPHI porosity logs. 


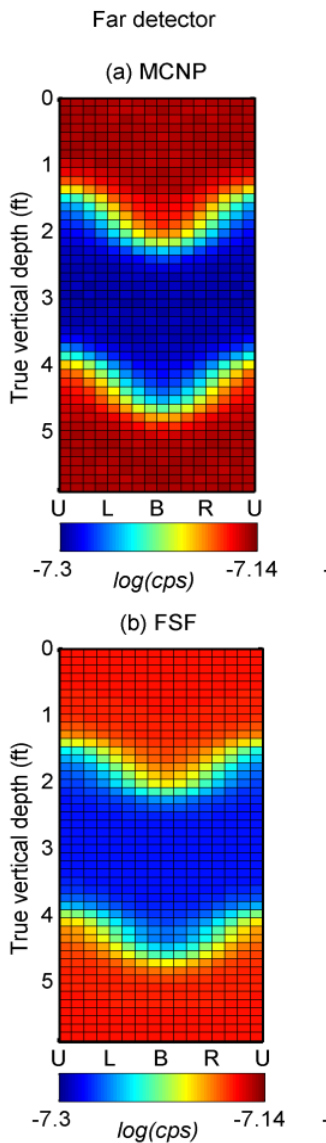

Near detector

(c) MCNP

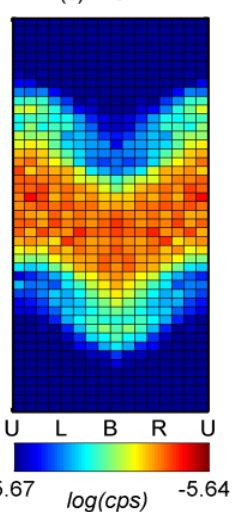

$\log (c p S)$
(d) FSF

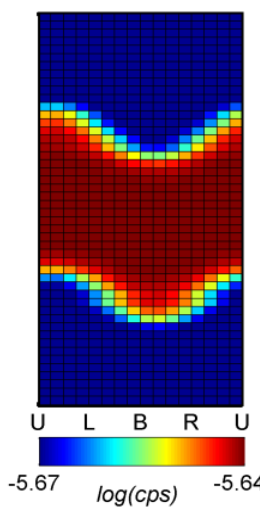

(e) $\mathrm{BPHI}_{\mathrm{MC}}$

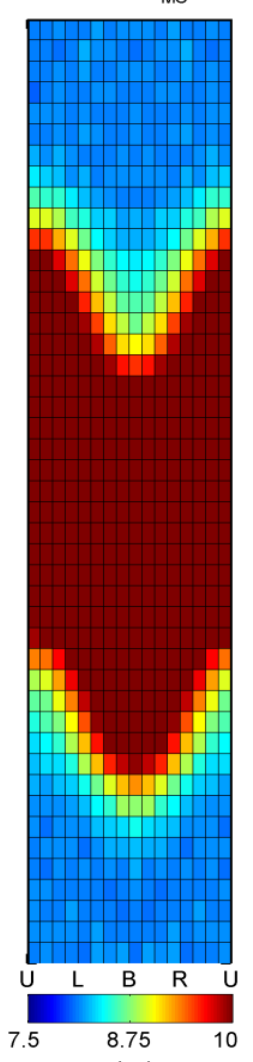

(pu) (f) $\mathrm{BPHI}_{\mathrm{FSF}}$

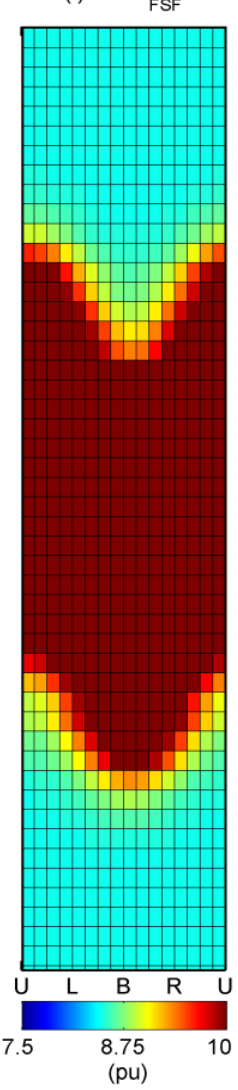

(g) $\rho_{\text {comp }}$

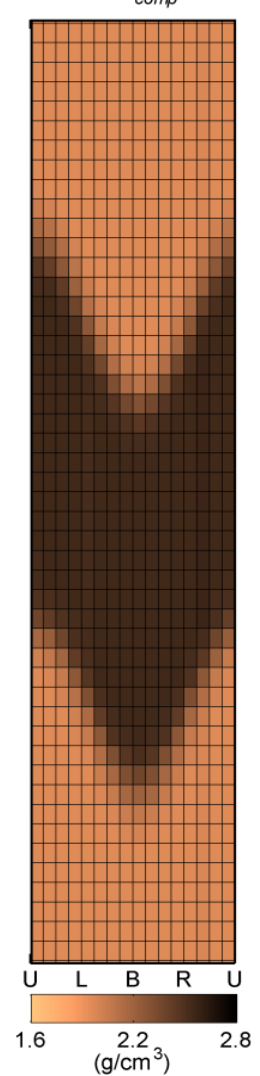

(h) Bottom logs

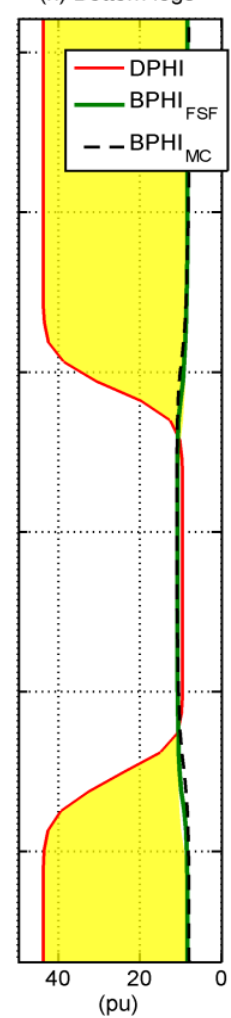

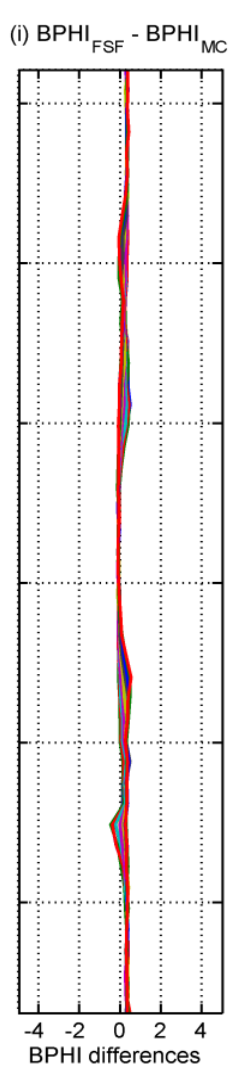

(pu)

Figure 2.12: Comparison of LWD MCNP and FSF- $F_{p}$ simulation results in an $89^{\circ}$ well across a synthetic earth model of $2.5-$ $\mathrm{ft}$ layer thicknesses. Logarithm of far detector counts obtained with (a) MCNP and (b) FSF- $F_{p}$ methods, versus true vertical depth. Logarithm of near detector counts obtained with (c) MCNP and (d) FSF- $F_{p}$ methods. BPHI neutron porosity image from (e) MCNP and (f) FSF- $F_{p}$ methods. (g) Simulated compensated density image. (h) Bottom quadrant DPHI and BPHI porosity logs. (i) BPHI sector differences between FSF and MCNP methods. 


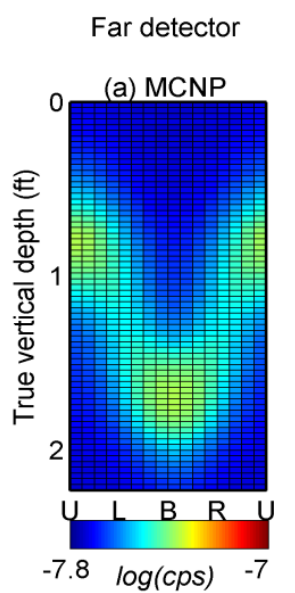

(b) FSF

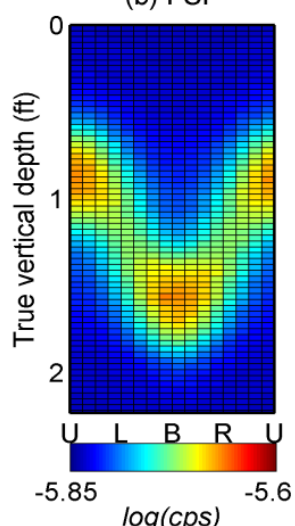

$\log (c p s)$
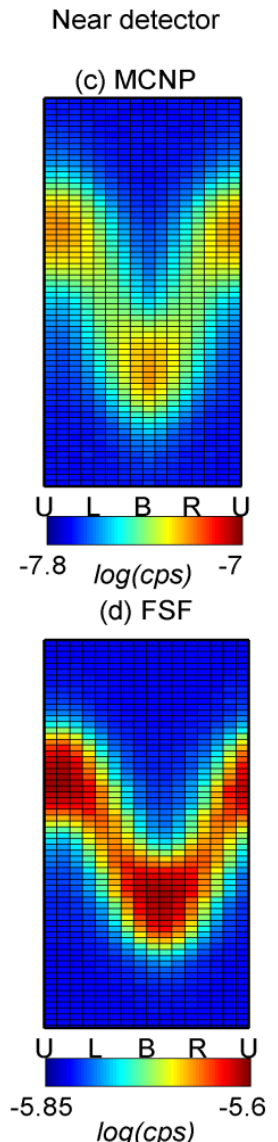

$\log (c p s)$ (e) $\mathrm{BPHI}{ }_{M C}$

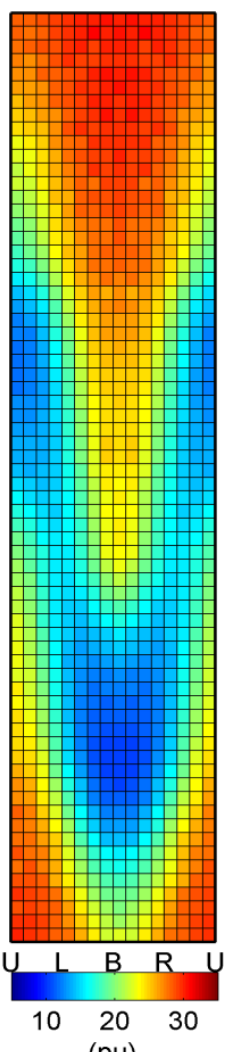

(f) $\mathrm{BPHI}_{\mathrm{FSF}}$

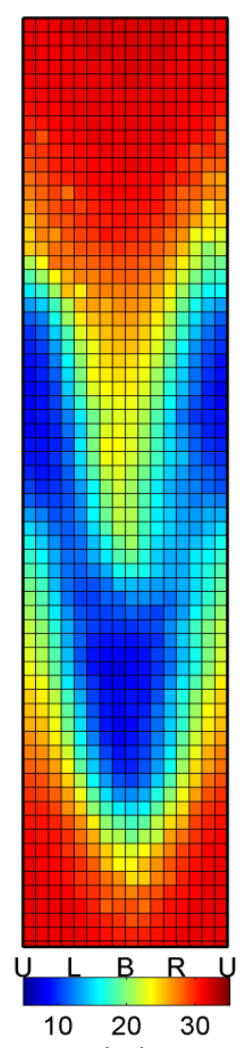

(g) $\rho_{\text {comp }}$

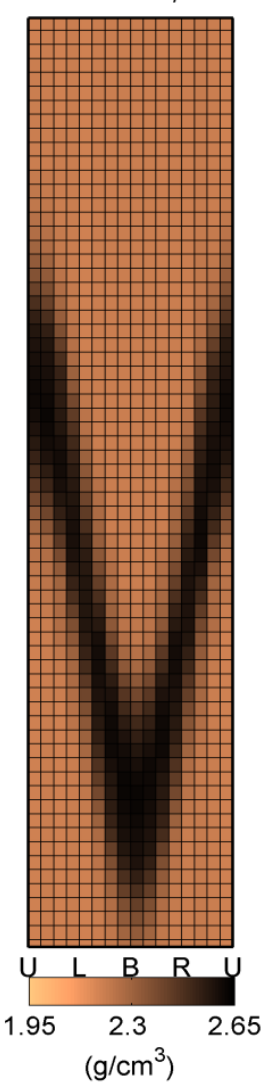

(h) Bottom logs

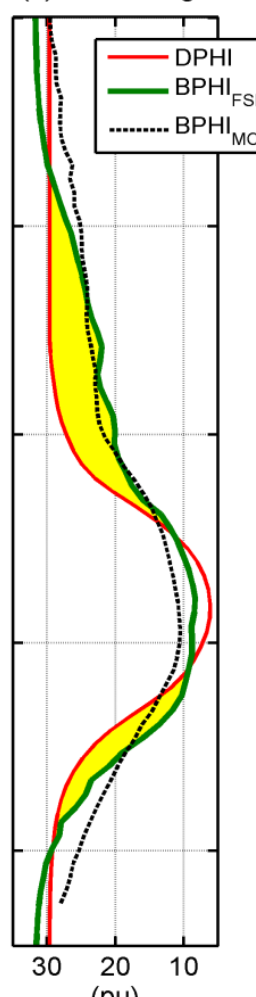

(pu) (i) $\mathrm{BPHI}_{\mathrm{FSF}}-\mathrm{BPHI}_{\mathrm{MC}}$

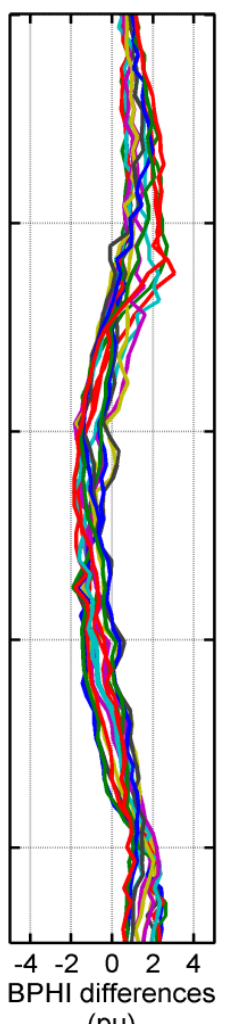

(pu)

Figure 2.13: Comparison of LWD MCNP and FSF- $F_{p}$ simulation results in an $85^{\circ}$ well across a synthetic earth model of 0.5 $\mathrm{ft}$ layer thickness. Logarithm of far detector counts obtained with (a) MCNP and (b) FSF- $F_{p}$ methods, versus true vertical depth. Logarithm of near detector counts obtained with (c) MCNP and (d) FSF- $F_{p}$ methods. BPHI neutron porosity image from (e) MCNP and (f) FSF- $F_{p}$ methods. (g) Simulated compensated density image. (h) Bottom quadrant DPHI and BPHI porosity logs. (i) BPHI sector differences between FSF and MCNP methods. 


\section{Chapter 3: Fast Modeling of Borehole Neutron Porosity Measurements with a New Spatial Transport-Diffusion Approximation}

The quantitative integration of nuclear measurements into the in-situ petrophysical and geophysical evaluation of rock formations has been elusive because of the lack of efficient numerical simulation algorithms. This chapter introduces a new method (second method considered in the first part of the dissertation) for rapid numerical simulation of borehole neutron measurements using Monte Carlo (MC)derived spatial flux sensitivity functions (FSFs) and diffusion flux-difference (DFD) approximations. The method calculates spatial sensitivity flux perturbations using fluxdifference approximations of one-group neutron diffusion models. By invoking appropriate boundary conditions, the one-group, time-independent neutron diffusion solution is implemented for non-multiplying systems in two- (2D) and three-dimensional (3D) cylindrical coordinates. The solution is differentiated with respect to neutron crosssection to obtain an expression for flux-difference due to cross-section perturbations. Constant transport-correction coefficients for cross-section parameters are calculated with a flux-fitting method to account for deviations of borehole neutron measurements from the physics of diffusion. Thereafter, spatial FSF responses are rapidly and accurately calculated using a first-order Rytov DFD approximation. Estimated flux-differences are next used to calculate lumped higher-order perturbation terms. The DFD technique is tested and benchmarked against MC calculations in the presence of tool standoff, invasion, and well deviation, for both wireline and logging-while-drilling (LWD) tools. Benchmark examples and application in highly-deviated wells indicate that neutron porosity logs can be accurately and efficiently simulated with the new DFD method, even in complex geometrical and physical conditions, with errors lower than 1.2 porosity units. 


\subsection{INTRODUCTION}

Reliable integrated petrophysical interpretation of nuclear logs has been elusive for a long time due to inefficient methods for fast and accurate simulation of borehole nuclear measurements. The traditional method for simulating borehole nuclear measurements is with the Monte Carlo N-particle transport code (MCNP, X-5 Monte Carlo Team, 2003). Albeit accurate and flexible, MCNP is computationally expensive and inefficient for inversion. Differential flux sensitivity methods (Watson, 1984, 1992; Couët et al., 1993; Case et al., 1994) that employ perturbation theory (Greenspan, 1976; Lewins, 1965) have proven to be the best option for fast and interactive modeling of nuclear measurements. The introduction of the concept of spatial flux sensitivity functions (FSFs, Mendoza et al., 2007) and the method of linear iterative refinement (Mendoza et al., 2010a, 2010b) prompted the development of inversion-based methods for petrophysical interpretation of wireline and logging-while-drilling (LWD) nuclear logs (Heidari et al., 2009, 2012; Ijasan et al., 2011; Mendoza et al., 2012). In comparison to MCNP, the linear iterative refinement (LIR) technique is several folds faster.

Guo et al. (2009) documented the application of a neutron diffusion code (Butler et al., 1992) that approximated the Boltzmann transport equation to rapidly simulate neutron logs. This approach exhibited limitations in the presence of shale layers (with high neutron absorbers) and across thin beds. At the same time, second-order calculation methods, such as Zhou et al. (2009) described for simulation of borehole gamma-gamma measurements, have opened alternate routes for fast modeling of nuclear borehole measurements.

The goal of LIR is to select a FSF with an equivalent detector response as that of the measurement in the presence of borehole environmental conditions, bed thickness, or/and bed dip. The technique works by iterative linear interpolation amongst Monte 
Carlo (MC)-derived FSFs, pre-calculated in homogeneous base-cases, such that the resultant FSF represents an equivalent detector response in a homogeneous formation. Applications of LIR indicated that its accuracy is primarily governed by the degree of variability and magnitude of perturbations encountered in rock formations while logging, particularly for neutron porosity simulations. The LIR employs first-order perturbations wherein rock variability introduces higher-order effects in the form of flux perturbations. Such degrees of variability are primarily driven by shoulder-bed effects, bed relative dip, mud-filtrate invasion, and borehole environmental properties. These situations occur frequently in measurements across high permeability and high porosity rocks where adequate petrophysical and geometrical modeling can improve the estimation of hydrocarbon reserves and flow quality. Across layers, invasion zones, or boreholeformation regions of significant contrasts or perturbations in neutron energy crosssection, FSFs exhibit significant flux perturbations which cannot always be adequately predicted by the LIR. This behavior yields inaccurate neutron porosity simulations in cases such as invasion of gas formations, washouts, and in high-angle/horizontal (HA/HZ) wells. A brute-force approach to circumventing this problem is to pre-calculate FSFs for a wide array of borehole environmental conditions, e.g., several borehole sizes, and several tool-binning orientations for sector LWD logs. The technical problems with this approach are longer computation time and larger storage disk space required by the calculations.

A similar situation of significant flux perturbations has been observed when modeling borehole sigma measurements across bed boundaries between sands and shales. Mimoun et al. (2011) showed that a diffusion model can be used to approximate such flux perturbations and hence improve the accuracy and reliability of borehole sigma 
models. Greenspan (1976) highlighted several other formulations for calculation of related flux perturbations.

In this chapter, I introduce a new rapid transport-diffusion approximation that accurately calculates spatial flux perturbations in the presence of complex formation geometries that give rise to significant contrasts in neutron energy cross-section or hydrogen index (HI). Such cases include mud-filtrate invasion, borehole standoff, and well deviation. I assume that the difference in flux due to cross-section perturbations from a background FSF (Monte Carlo-derived for a homogeneous base-case) can be approximated by the physics of neutron diffusion. Likewise, to account for neutron scattering collisions (Dworak et al., 2001) in the method, I introduce transport-correction coefficients in the diffusion model. The method enables the calculation of neutron diffusion flux-differences (DFDs) as approximations to FSF perturbations, hence improves the calculation of raw detector responses and modeling of neutron porosity measurements, particularly in complex geometrical and physical conditions. The DFD method is benchmarked with full MCNP calculations when performing qualitative and quantitative comparisons of perturbed FSFs and neutron porosity responses.

\subsection{METHOD}

The basis of sensitivity functions originates from importance calculations relating to the solution of the time-independent Boltzmann integro-differential adjoint transport equation (Greenspan, 1976), given by

$$
\begin{aligned}
{\left[-\boldsymbol{\Omega} \cdot \nabla+\Sigma_{t}(\mathbf{r}, E)\right] } & \Psi_{R}^{+}(\mathbf{r}, E, \boldsymbol{\Omega})= \\
& \int d E^{\prime} \int d \mathbf{\Omega}^{\prime} \Sigma_{s}\left(\mathbf{r}, E \mathbf{\Omega} \rightarrow E^{\prime} \mathbf{\Omega}^{\prime}\right) \Psi_{R}^{+}\left(\mathbf{r}, E^{\prime}, \mathbf{\Omega}^{\prime}\right),
\end{aligned}
$$


where $\Psi_{R}^{+}$is the adjoint flux at position $\mathbf{r}$ of energy $E$ and direction $\Omega$ with respect to a detector at observation point $\mathbf{r}_{R}, \Sigma_{t}$ is total macroscopic energy cross-section (i.e., $\left.\Sigma_{t}=\Sigma_{a}+\Sigma_{s}\right), \Sigma_{s}$ is macroscopic scattering energy cross-section, and $\Sigma_{a}$ is macroscopic absorption energy cross-section. Below, I introduce a concise diffusion approximation of equation 3.1 to improve the efficiency of its numerical solution.

\subsubsection{Diffusion Formulation}

I assume that FSF perturbations due to changes in formation HI from a homogeneous background case, $B$, can be described by diffusion. Therefore, I invoke a modified one-group, time-independent neutron diffusion model for non-multiplying systems (Tittle, 1961) given by

$$
D\left[\nabla^{2}-\alpha^{2}\right] \psi_{R}\left(\mathbf{r}, \mathbf{r}_{S}, \alpha\right)=S_{o} \delta\left(\mathbf{r}-\mathbf{r}_{S}\right),
$$

where $D$ is the thermal diffusion coefficient, $\nabla^{2}$ is Laplace's operator, $\alpha$ is a modified neutron cross-section parameter, $\psi_{R}$ is spatial diffusion scalar flux at observation location $R$ in particles per second per unit area, $S_{o}$ is source strength in particles per second per unit volume, and $\delta$ is Dirac's delta function. The two-dimensional (2D) solution of equation 3.2 in cylindrical coordinates, where $\mathbf{r}=\mathbf{r}(r, z)$ and $\mathbf{r}_{S}=\mathbf{r}_{S}\left(r_{S}, z_{S}\right)$, gives rise to the Green's function kernel, $G_{\alpha}$ (Mandelis, 2001), due to a neutron point source of unity strength and located at $\mathbf{r}_{S}$, namely,

$$
\begin{array}{r}
G_{\alpha}\left(\mathbf{r}, \mathbf{r}_{S}\right)=\frac{1}{2 \pi D r_{e x}^{2}} \sum_{n} \frac{J_{0}\left(\beta_{n} r / r_{e x}\right) J_{0}\left(\beta_{n} r_{s} / r_{e x}\right)}{\kappa_{n} J_{1}^{2}\left(\beta_{n}\right)} e^{-\kappa_{n}\left|z-z_{s}\right|} \\
0 \leq r, r_{s} \leq r_{e x},
\end{array}
$$


where $n$ is the $n$-th eigensolution, $J_{0}$ and $J_{1}$ are zero- and first-order Bessel functions of the first kind, $\beta_{n}$ is $n$-th zero (root) of $J_{0}$, i.e., $J_{0}\left(\beta_{n}\right)=0, \kappa_{n}$ is $\sqrt{\alpha^{2}+\left(\beta_{n} / r_{e x}\right)^{2}}$, and $r_{e x}$ is extrapolation radial length for which the Green's function $G_{\alpha}=0$ when $r=r_{e x}$. The solution in equation 3.3 is differentiated with respect to the cross-section parameter, $\alpha$, to obtain a diffusion sensitivity function, given by

$$
J_{\alpha}\left(\mathbf{r}, \mathbf{r}_{S}\right)=\frac{-1}{2 \pi D r_{e x}^{2}} \sum_{n} \frac{\alpha J_{0}\left(\beta_{n} r / r_{e x}\right) J_{0}\left(\beta_{n} r_{s} / r_{e x}\right)}{\kappa_{n}^{3} J_{1}^{2}\left(\beta_{n}\right)}\left(1+\kappa_{n}\left|z-z_{s}\right|\right) e^{-\kappa_{n}\left|z-z_{s}\right|}
$$

The Green's function kernel and its derivative, $G_{\alpha}$ and $J_{\alpha}$ in equations 3.3 and 3.4 serve as the building blocks for spatial transport-diffusion flux-difference approximations due to cross-section perturbations, $\Delta \alpha$, in a homogeneous background case with crosssection parameter, $\alpha_{B}$. Figure 3.1 shows calculated diffusion kernels for $\alpha_{B}^{-1}$ equal to 30 $\mathrm{cm}$. It follows that the space integrated diffusion flux-difference function at $\mathbf{r}$, due to a response distributed source in $B$ at $\mathbf{r}_{S}$, for a detector located at $\mathbf{r}_{R}$ is given by

$$
\frac{J_{B}}{\psi_{B}}=\frac{\int d \mathbf{r}_{S} F S F_{B}\left(\mathbf{r}_{R}, \mathbf{r} ; \mathbf{r}_{S}\right) J_{\alpha, B}\left(\mathbf{r} ; \mathbf{r}_{S}\right)}{\int d \mathbf{r}_{S} F S F_{B}\left(\mathbf{r}_{R}, \mathbf{r} ; \mathbf{r}_{S}\right) G_{\alpha, B}\left(\mathbf{r} ; \mathbf{r}_{S}\right)},
$$

where $F S F_{B}$ is the MC-derived FSF in $B$, representing the source distribution, such that $\int d \mathbf{r}_{S} F S F_{B}\left(\mathbf{r}_{R}, \mathbf{r} ; \mathbf{r}_{S}\right)=1$, with $G_{\alpha, B}$ and $J_{\alpha, B}$ calculated from equations 3.3 and 3.4, respectively. Using a first-order Rytov approximation (Habashy et al., 1993), the spatial neutron DFD due to perturbation $\Delta \alpha$ can be written as

$$
\log _{e} F S F_{D F D}-\log _{e} F S F_{B}=\frac{J_{B}}{\psi_{B}} \Delta \alpha
$$


where $F S F_{D F D}$ is the DFD perturbed spatial sensitivity flux in a formation model with parameter $\alpha$, and $\Delta \alpha$ is the formation perturbation given as $\alpha-\alpha_{B}$.

The Green's function kernels, $G_{\alpha}$ and $J_{\alpha}$ in equations 3.3 and 3.4 represent 2D cylindrical coordinate geometry, i.e., $\mathbf{r}=\mathbf{r}(r, z)$. The diffusion formulation is conveniently extended to three-dimensional (3D) cylindrical geometry, i.e., $\mathbf{r}=\mathbf{r}(r, z, \theta)$, by using Green's function solutions in spherical coordinates, i.e., $\mathbf{r}=\mathbf{r}(r, \theta, \phi)$, and a coordinate transformation. In spherical coordinates, $G_{\alpha}$ and $J_{\alpha}$ are given by

$$
G_{\alpha}=\frac{1}{4 \pi D\left|\mathbf{r}-\mathbf{r}_{S}\right|} e^{-\alpha\left|\mathbf{r}-\mathbf{r}_{S}\right|},
$$

and

$$
J_{\alpha}=\frac{-1}{4 \pi D} e^{-\alpha\left|\mathbf{r}-\mathbf{r}_{s}\right|}
$$

respectively. In the kernel solution, $\left|\mathbf{r}-\mathbf{r}_{S}\right|$ is the distance between source location and observation point. The transformation from cylindrical to spherical coordinates is given by

$$
\left|\mathbf{r}-\mathbf{r}_{S}\right|^{2}=r^{2}+r_{S}^{2}-2 r r_{S} \cos \left(\theta-\theta_{S}\right)+\left(z-z_{S}\right)^{2},
$$

such that equations 3.7 and 3.8 can be applied to 3D cylindrical geometry. Equations 3.7 - 3.9 describe the 3D kernel functionality for 3D DFD calculations in the presence of 3D model perturbations, e.g. deviated beds, thinly bedded formations, eccentered tool geometry, non-circular borehole, and non-borehole-symmetric invasion in $\mathrm{HA} / \mathrm{HZ}$ wells, among others. 


\subsubsection{Boundary Conditions and Flux Continuity}

At formation boundaries or/and invasion fronts, $\mathbf{r}_{b}$, where $\Delta \alpha\left(\mathbf{r}_{b}+\varepsilon\right) \neq \Delta \alpha\left(\mathbf{r}_{b}-\varepsilon\right)$ for $\varepsilon \rightarrow 0$, flux continuity is ensured by the condition

$$
\left.\frac{J_{B}}{\psi_{B}} \Delta \alpha\right|_{\mathbf{r}=\mathbf{r}_{b}-\varepsilon}=\left.\frac{J_{B}}{\psi_{B}} \Delta \alpha\right|_{\mathbf{r}=\mathbf{r}_{b}+\varepsilon} \forall \mathbf{r}>\mathbf{r}_{S} .
$$

Note that this condition is driven by $\Delta \alpha$ and not $F S F_{B}$, since $F S F_{B}$ is derived in homogeneous formations. It follows that, $F S F_{B}$ 's DFD distributed function, $J_{B} / \psi_{B}$, can be reused for several magnitudes and distributions of $\Delta \alpha$, thereby saving computational time.

\subsubsection{Transport Correction}

One-group neutron transport, from source to detection in well-logging applications, is primarily composed of an asymptotic/diffusion part and a transient part (Dworak et al., 2001). The transient part, consisting of neutron scattering collisions and higher-order interaction terms, introduces a departure from a full diffusion problem, particularly across high neutron absorbers where the transient part dominates the total flux. Assumptions implicit in diffusion theory, such as isotropic particle transport (Stacey, 2007), are inadequate to represent such higher-order interaction effects. An approximate modification is in order. Butler et al. (1992) showed that the coefficients of a diffusion model can be systematically modified to reproduce higher-order transport effects resulting from the transient part of neutron transport.

In my DFD model, departures from a full diffusion solution due to higher-order transport effects are partially accounted for in MC-derived $F S F_{B}$, as shown in equation 3.6. Also, the neutron diffusion model is modified such that 


$$
\alpha=\frac{1}{L_{l E}^{\prime}}=\frac{1}{\eta L_{m}}
$$

where $L_{1 E}^{\prime}$ is a modified one-group travel length, $L_{m}$ is formation migration length, and $\eta$ is the transport-correction coefficient. Neutron characteristic lengths, i.e., diffusion length $\left(L_{d}\right)$, slowing-down length $\left(L_{s}\right)$, and migration length $\left(L_{m}\right)$ are calculated with Schlumberger Nuclear Parameter calculator (SNUPAR, McKeon et al., 1989). Accordingly, the transport-correction coefficient, $\eta$, is calculated by flux-fitting onedimensional (1D) radial FSFs, MC-derived in homogeneous base-cases across various formation lithologies, to the Green's function solutions of equations 3.3 and 3.4. The systematic flux-fitting method, described in Figure 3.2, implements a nonlinear optimization approach to select the transport-correction coefficient, $\eta$, that minimizes the misfit between the perturbed DFD solution and MC-derived fluxes. By adjusting the neutron cross-section in terms of $\eta$, this strategy causes the diffusion model to account for higher-order transport effects resulting from the transient part of neutron transport.

\subsubsection{Detector Response Perturbation}

The Taylor's series expansion for estimating detector response perturbations (X-5 Monte Carlo Team, 2003) is given by

$$
\Delta N^{m}\left(\mathbf{r}_{R}\right)=N_{B}\left(\mathbf{r}_{R}\right) \sum_{m=1}^{\infty} \int d \mathbf{r} \frac{1}{m !} \frac{d^{m} N}{d \alpha^{m}}\left(\mathbf{r}_{R}, \mathbf{r}\right)\left(\frac{\Delta \alpha(\mathbf{r})}{\alpha_{B}(\mathbf{r})}\right)^{m}
$$


where $\Delta N^{m}$ is the $m$-th order change in detector response at detector location $\mathbf{r}_{R}, \frac{1}{m !} \frac{d^{m} N}{d \alpha^{m}}$ is the $m$-th order response sensitivity coefficient, $N_{B}$ is the background detector response, and $N$ is the perturbed detector response due to $\Delta \alpha$. It follows that

$$
N\left(\mathbf{r}_{R}\right)=N_{B}\left(\mathbf{r}_{R}\right)+\Delta N^{m}\left(\mathbf{r}_{R}\right) .
$$

The DFD technique aims to estimate the contribution of higher-order terms $(m>1)$ in equation 3.12 through a diffusion model. Upon prediction of $F S F_{D F D}$, as in equation 3.6, lumped higher-order perturbation calculations are carried out to estimate detector responses before calibration to neutron porosity, i.e.,

$$
\begin{aligned}
N\left(\mathbf{r}_{R}\right)=N_{B}\left(\mathbf{r}_{R}\right)+ & N_{B}\left(\mathbf{r}_{R}\right) \int d \mathbf{r} \operatorname{FSF}_{B}\left(\mathbf{r}_{R}, \mathbf{r}\right) \frac{\Delta \alpha}{\alpha_{B}} \\
& +N_{B}\left(\mathbf{r}_{R}\right) \int d \mathbf{r} \Delta F S F\left(\mathbf{r}_{R}, \mathbf{r}\right) \frac{\alpha}{\alpha_{B}},
\end{aligned}
$$

where $\triangle F S F$ is the flux perturbation $\left(F S F_{D F D}-F S F_{B}\right)$ due to $\Delta \alpha$. The second term on the right-hand side (RHS) of equation 3.14 is the first-order perturbation term, while the third term is a lumped higher-order perturbation term that accounts for flux perturbation effects. It is worth noting that effects due to higher-order terms and flux perturbations are minimized when $\Delta \alpha \ll \alpha_{B}$, a condition LIR aims to achieve so that the third term on the RHS of equation 3.14 is negligible, whereby a first-order perturbation is an accurate representation of the physics of the problem. The selection of a $F S F_{B}$ such that $\Delta \alpha$ is minimized improves the accuracy of the perturbation calculation, especially in the case of 
LIR. For highly perturbed situations, first-order perturbations are inadequate and LIR becomes inaccurate.

\subsection{NUMERICAL RESULTS}

I implement the rapid DFD method described above for two generic neutronneutron tool models:

(1) a dual-detector wireline neutron tool with an americium-beryllium (AmBe) neutron source in a 3.0-in. (7.6-cm) tool sonde (Mendoza et al., 2007), and

(2) a dual-detector LWD neutron tool with a deuterium-tritium (DT) $14 \mathrm{MeV}$ neutron source in an 8.5 -in. $(22-\mathrm{cm})$ drill collar. Similarly, density measurements are numerically simulated using a generic dual-detector LWD gamma-gamma tool (Ijasan et al., 2011).

\subsubsection{Transport-Correction Coefficients, $\eta$}

Using the flux-fitting method shown in Figure 3.2, $\eta$ is calculated for far and near detectors of the generic tools described above. The DFD model is re-computed in successive iterations, as shown in Figure 3.2, until reaching the lowest possible 1D FSF misfit between the DFD model and MC calculations. The modified travel length, $L^{\prime}{ }_{I E}$, that minimizes the flux misfit is output for each formation base-case. For robustness, 1D FSFs in base-cases of water- and gas-filled lithologies (sandstone, limestone, and dolomite) are used in the calculations. Figure 3.3 shows cross-plots of $L_{I E}^{\prime}, L_{d}$, and $L_{m}$, for the wireline and LWD models. In the modified diffusion model, the equivalent neutron mean-free-path traveled is greater because of scattering interactions, therefore $L_{I E}^{\prime}$ is greater than $L_{d}$ (Figure 3.3, left panels). This behavior implies that transient effects originate apparent cross-sections smaller than the diffusion cross-section (Ellis and Singer, 2007). Comparison of $L_{I E}^{\prime}$ to $L_{m}$ (Figure 3.3, right panels) indicates minimal 
scatter plot of a one-group calculation. In addition to transport effects, $\eta$ (see Table 3.1) depends on tool design and detector spacing because the FSF is dependent on the detector $\mathbf{r}_{R}$, i.e., $\eta=\eta\left(\mathbf{r}_{R}\right)$. Figure 3.3 shows that $\eta$ is approximately constant per detector across various formation lithologies and fluid constituents for both wireline and LWD tools. It is also worth noting that $1 / \eta$ is larger for a $14 \mathrm{MeV}$ LWD tool than for an AmBe wireline tool (Table 3.1), because faster neutrons require larger corrections to the neutron crosssection, $\alpha$ (equation 3.11). The modified one-group travel length, $L_{1 E}^{\prime}$, is a formation parameter representative of the lumped transport-diffusion model. It describes the effective mean-free-path traveled by neutrons from their generation to their detection per detector and per tool model. It also serves as modification to the diffusion theory where I account for scattering interactions.

\subsubsection{Diffusion Distributed Functions in Homogeneous Formations}

I observe homogeneous perturbations in neutron cross-sections as porosity or/and fluid constituents vary in base-case formations. Using the MC-derived background FSF, $F S F_{B}$, calculated in a limestone block with an LWD tool, I illustrate the rapid calculation of the perturbed $F S F_{D F D}$ (using DFD model) due to homogeneous formation perturbations and compare to $F S F_{M C}$ (full MCNP calculations). In this exercise, the 2D kernel functionality (equations 3.3 and 3.4) in the Rytov DFD model (equations 3.5 and $3.6)$ is adequate to calculate the required properties of the $F S F_{D F D}$.

Figure 3.4 shows the normalized 2D forward and derivative diffusion distributed functions, $\psi_{B}$ and $J_{B}$, respectively, for near and far detectors of the LWD tool across a limestone block. The $\psi_{B}$ functions describe detector diffusion fluxes with a distributed neutron source defined by the FSF maps, $F S F_{B}$ (panels a and e). Qualitative comparison of $\psi_{B}$ and $F S F_{B}$ emphasizes the isotropic nature of the diffusion model. Panels $\mathrm{c}$ and $\mathrm{g}$ 
show derivative diffusion distribution functions, $J_{B}$. Intuitively, neutron flux responses decrease in magnitude as $\mathrm{HI}$ increases, whereby $J_{B}$ becomes negative. The DFD functions, $J_{B} / \psi_{B}$ shown in panels $\mathrm{d}$ and $\mathrm{h}$ measure the logarithmic change of detector FSFs with respect to limestone cross-section, i.e., the Rytov functional formulation in equation 3.6. It also measures the range and volume of formation wherein perturbations will influence detector responses. Normalized 1D projections of the flux functions, shown in white, are superimposed to the plots shown in Figure 3.4. The 1D vertical projections are obtained by integrating the FSF in radial and azimuthal directions, while 1D azimuthal projections are obtained by integrating the FSF in vertical and radial directions. Similarly, 1D radial projections are obtained by integrating the FSF in vertical and azimuthal directions, whereas the radial J-factor is a cumulative function of the 1D radial projection. Figure 3.4 shows 1D vertical projections and radial J-factors.

The workflow diagram in Figure 3.5 summarizes the DFD calculation algorithm, described in the method and equations 3.3 - 3.14, for simulation of neutron near-to-far ratio porosity, $\phi_{N}$. The workflow highlights pertinent aspects of the DFD model for forward modeling of $\phi_{N}$ measurements as discussed in this chapter. I apply the DFD method using diffusion distributed functions shown in Figure 3.4 to estimate flux perturbations due to homogeneous perturbations in the limestone block (as indicated in equation 3.6). Qualitatively, 1D FSF radial and vertical projections are compared despite using the 2D kernel functionality. Figure 3.6 compares the perturbed $F S F_{D F D}$ to $F S F_{M C}$ (full MCNP calculations) in water- and gas-filled base-case formations perturbed from the limestone block (see Table 3.2 for base-case descriptions). I observe that the DFD method accurately reproduces full MCNP calculations as formation porosity and/or fluid constituents vary. For the cases shown in Figure 3.6, MCNP calculations required approximately 300 minutes of $\mathrm{CPU}$ (computer processing unit) time per base-case on a 
Linux cluster with Intel Xeon 6-core $2.93 \mathrm{GHz}$ processors, while the DFD method required 0.160 seconds on a Windows XP PC with dual-core $3.2 \mathrm{GHz}$ processors. Note that $J_{B}$ and $\psi_{B}$ for the limestone block (which characterize and anticipate perturbation effects in DFD model) are calculated only once, thus significantly decreasing the corresponding CPU time. An advantage of the DFD technique is that several base-case FSF libraries can be rapidly generated with only a few background FSFs, without the need of extended MCNP calculations.

\subsection{BenCHMARK ReSUlts: Borehole AND ENVIRONMENTAL CONDITIONS}

Measurements of in-situ formation properties can be deleteriously affected by washouts, borehole standoff, and mud-filtrate invasion in open-hole logging. The severity of these effects depends on the duration of formation exposure to borehole environmental conditions before setting casing. In the presence of borehole environmental effects, the HI contrast between borehole and formation causes significant FSF perturbations, especially at low values of formation porosity. This section benchmarks LIR and DFD calculations of $\phi_{N}$, due to standoff and invasion perturbations, against full MCNP calculations. The borehole is assumed to be filled with fresh water.

\subsubsection{Borehole Standoff Effects in LWD Measurements}

During measurement acquisition in $\mathrm{HA} / \mathrm{HZ}$ wells, LWD tools tend to stand at the bottom of the hole because of gravity. This behavior gives rise to $3 \mathrm{D}$ tool eccentricity effects such that standoff is non-symmetric around the borehole. Hence, differing degrees of standoff originate for different tool azimuthal orientations. These effects are further complicated in non-circular and washed-out boreholes. On account of such effects, the 3D DFD functionality, as described in equations $3.7-3.9$, is implemented for the case of LWD standoff perturbations. 
Assuming the 8.5-in-collar LWD neutron tool across base-cases described in Table 3.2, in a 12-in. (30-cm) borehole, $\phi_{N}$ errors for both DFD and LIR are estimated for three sector binning orientations: bottom (B), left (L), and up (U). With the LWD tool collar standing at the bottom of the borehole, zero standoff is observed at the B sector orientation, while 3.5 in. $(8.9 \mathrm{~cm})$ maximum standoff is observed at the $U$ sector orientation. Figure 3.7 shows the corresponding $\phi_{N}$ errors for three sector orientations, while spatial 3D FSFs using $\operatorname{MCNP}\left(F S F_{M C}\right)$, DFD $\left(F S F_{D F D}\right)$, and LIR $\left(F S F_{L I R}\right)$ in LIME05W base-case (see Table 3.2), for bottom and up orientations, are qualitatively compared in Figures 3.8 and 3.9.

At the B sector orientation, LIR $\phi_{N}$ errors (Figure 3.7, panel a) are approximately 3 pu primarily because $F S F_{L I R}$ fails to match full MC-derived $F S F_{M C}$ (Figure 3.8). This behavior is evident in the 1D azimuthal projections shown in panels e and $\mathrm{j}$ of Figure 3.8. Due to the broad azimuthal aperture of neutron responses (Ijasan et al., 2011), standoff crevices at the $\mathrm{B}$ sector contribute significantly to porosity sensitivity. Scattering collisions in the crevices result in a more tapered FSF in comparison to a slick borehole. This tapering, evident in the azimuthal projection of $F S F_{M C}$, is adequately reproduced by $F S F_{D F D}$. At the $U$ sector orientation (Figure 3.9), discrepancies between $F S F_{L I R}$ and $F S F_{M C}$ are more conspicuous in the $1 \mathrm{D}$ radial and azimuthal projections (panels c, h, e, and j). Standoff effects on FSFs are adequately reproduced in $F S F_{D F D}$, due to lumped higher-order perturbation, thereby resulting in DFD $\phi_{N}$ errors lower than $1 \mathrm{pu}$ (Figure 3.7). The significant LIR $\phi_{N}$ errors are due to inadequacy of first-order perturbation to quantify standoff effects. 


\subsubsection{Invasion Effects in Wireline Measurements}

Invasion effects are investigated by assuming a wireline tool in an 8-in. (20-cm) borehole, and fresh-water invasion into a 30\% gas-filled limestone formation, with zero residual gas saturation. The saturating gas is assumed to be methane $\left(\mathrm{CH}_{4}\right)$ of density $0.0178 \mathrm{~g} / \mathrm{cm}^{3}$. Additionally, piston-like radial invasion is assumed such that invaded and virgin zones consist of 30\% water- and gas-filled limestone formations, respectively; $L_{m}$ values calculated with SNUPAR $(12.20 \mathrm{~cm}$ and $34.60 \mathrm{~cm}$ for the invaded and virgin zones, respectively) indicate a significant contrast between invaded and virgins zones. In this benchmark example, MCNP, LIR, and DFD calculations are used to simulate neutron porosity measurements for 25 instances of radial invasion length into the formation.

Figure 3.10 shows quantitative and qualitative comparisons of detector FSFs, count rates, and calibrated neutron porosity; panels in the second and third columns display 1D FSF radial and vertical projections when the invasion front is $1.15 \mathrm{in} ., 2.85 \mathrm{in} ., 6.81 \mathrm{in}$., and 10.38 in. $(2.91 \mathrm{~cm}, 7.23 \mathrm{~cm}, 17.30 \mathrm{~cm}$, and $26.37 \mathrm{~cm})$ from the borehole wall. Panels c and $\mathrm{d}$ in the first column show $\phi_{N}$ measurements and errors, respectively, for all 25 instances of invasion front.

Similar to standoff perturbations, FSFs obtained with LIR in this invasion benchmark example are biased by formation properties near the borehole. As observed in panels $\mathrm{g}$ and h of Figure 3.10, $F S F_{L I R}$ matches $F S F_{M C}$ only in the invaded zone, while $F S F_{D F D}$ matches $F S F_{M C}$ in both invaded and virgin zones. This is the main source of error in detector measurements calculated with LIR. The maximum $\phi_{N}$ error with LIR is $6.8 \mathrm{pu}$ while the error with the DFD approximation is $1.1 \mathrm{pu}$. It is worth noting that DFD results for the 25 invasion fronts were obtained with only three base-case $F S F_{B}$ - LIME30G for invasion fronts shorter than $3.25 \mathrm{in} .(8.25 \mathrm{~cm})$, LIME05W for invasion fronts shorter than 6.81 in. $(17.30 \mathrm{~cm})$ but longer than 3.25 in., and LIME30W for invasion fronts longer 
than 6.81 in. from the borehole wall (see Table 3.2 for a summary of base-case properties). The base-cases are selected by volumetrically averaging the formation $L_{m}$ first and subsequently picking the closest $F S F_{B}$ in the library set which corresponds to the averaged $L_{m}$ (see Figure 3.5) and such that it minimizes $\Delta \alpha$ in equation 3.6.

\subsection{Modeling Applications in High-Angle and Horizontal Wells}

Reliable integrated petrophysical interpretation of nuclear logs requires fast, efficient, and accurate modeling workflows and inversion methods for improved confidence in inferred petrophysical properties. HA/HZ wells, in comparison to vertical wells, give rise to more pronounced shoulder-bed effects and significant formation variability at bed boundaries. They introduce significant formation perturbations while logging, especially across beds of large contrast in $\mathrm{HI}$ and in the presence of borehole environmental effects.

Using the DFD method discussed above and invoking a LWD neutron tool model, I perform simulation of neutron measurements across layers in a synthetic earth model penetrated by an $85^{\circ}$ well. Azimuthal LWD measurements, for complete tool rotation, are binned assuming a 16-sector binning scheme, and logged at $0.125 \mathrm{ft}(3.81 \mathrm{~cm})$ per sample in true vertical depth. Tables 3.3 and 3.4 describe the corresponding layer matrix and fluid constituents in the earth model. The 3D kernel functionality described in equations 3.7 - 3.9 is used to calculate 3D DFD perturbations for the synthetic example in the presence of shoulder-bed effects, standoff, and invasion. Results are compared to LIR and full MCNP calculations.

\subsubsection{Synthetic Case of a High-Angle Well in a Slick Borehole}

Figure 3.11 compares neutron porosity images $\phi_{N-M C}, \phi_{N-D F D}$, and $\phi_{N-L I R}$ in sandstone pu calculated with MCNP, DFD, and LIR, respectively, for the synthetic model 
described in Table 3.3. Layer true stratigraphic thickness (TST) is $1.3 \mathrm{ft}(40 \mathrm{~cm})$ and the tool collar is slick in the borehole with no standoff. The MC-simulated wireline neutron $\log , \phi_{N, W L-M C}$ (dashed black line) is included in panel f for qualitative comparisons. Also shown is the simulated LWD compensated density, $\rho_{C O-L I R}$ assuming the generic LWD gamma-gamma tool described earlier. Panel f (lower left) shows bottom, B sector neutron and density logs simulated in the $85^{\circ}$ well across the synthetic model. The brown shaded zones, layer I of Table 3.3, consist of 5\% porosity shale matrix. In this layer, neutron $\operatorname{logs}\left(\phi_{N-M C}, \phi_{N-D F D}, \phi_{N, W L-M C}\right.$, and $\left.\phi_{N-L I R}\right)$ show a significantly higher apparent porosity than the density $\log \left(\rho_{C O-L I R}\right)$ - characteristic of shale layers. The yellow shaded zones, layers II and III of Table 3.3, consist of gas-filled sandstone. Simulated neutron porosities are smaller than simulated apparent density porosities (calculated from the simulated density $\log$ ), thereby giving rise to the characteristic crossover of gas-saturated layers. In layer IV, consisting 30\% water-filled sandstone, a false gas crossover is observed because of shoulder-bed effects. This behavior is typical across highly-deviated beds because of differing volumes of investigation (VOI) of density and neutron measurements. I observe an overlap of neutron and density logs in layer $\mathrm{V}$, consisting of $5 \%$ water-filled sandstone.

Panels $g$ and h of Figure 3.11 show the porosity errors of $\phi_{N-L I R}$ and $\phi_{N-D F D}$ in comparison to $\phi_{N-M C}$, respectively; average porosity residuals, per depth and azimuth, are shown in panels $\mathrm{i}$ and $\mathrm{j}$, respectively. I observe that $\phi_{N-L I R}$ yields large errors, as high as 8 pu, at measurement locations affected by shoulder beds. On the other hand, because the DFD method accurately calculates flux perturbations, $\phi_{N-D F D}$ exhibits improved neutron porosity as the tool transverses across bed boundaries. Figures 3.12 and 3.13 show 3D FSFs obtained from DFD and MCNP at depths in the B sector orientation of the well where the $\phi_{N-L I R}$ errors are the largest, i.e. at bed boundaries with pronounced formation 
variability. In these figures, I juxtapose $1 \mathrm{D}$ vertical and radial projections of $F S F_{M C}$, $F S F_{D F D}$, and $F S F_{L I R}$ (panels c, d, g, and h) for qualitative comparisons; FSFs calculated with the 3D DFD technique agree with MCNP hence the decreasing error in $\phi_{N-D F D}$.

\subsubsection{Synthetic Case of a High-Angle Well with Invasion Effects}

Dynamic petrophysical conditions of invaded formations introduce significant perturbations in the form of complex geometries, especially in $\mathrm{HA} / \mathrm{HZ}$ wells. Due to gravity segregation of invading and in-situ fluids in $\mathrm{HA} / \mathrm{HZ}$ wells, the spatial distribution of mud filtrate tends to be non-symmetric around the perimeter of the borehole, i.e., longest at the bottom, and gradually shortest toward the top of the borehole. This phenomenon is usually referred to as 3D "teardrop" invasion profile.

Assuming the synthetic earth model described in Table 3.3, approximate "teardrop" invasion profiles are modeled as 3D eccentric cylinders within bed layers. Oilbased mud (OBM, $\left.\mathrm{C}_{14} \mathrm{H}_{30} 0.8 \mathrm{~g} / \mathrm{cm}^{3}\right)$ is assumed as the invading borehole fluid, while bed layer residual fluid saturations are assumed to be $0 \%$. The radial length of invasion is also assumed to be inversely proportional to layer pore volume, and dependent on matrix type. For example, the $30 \%$ porosity sand layers III and IV exhibit shallower invasion depths than the $5 \%$ porosity layers II and V. The shale layer I has the shallowest depth of invasion. Table 3.4 describes the assumed invasion profiles for each of the layers included in this model.

Figure 3.14 shows the geometry of invasion profiles and LWD (neutron and density) simulation results. The contrast between invaded and virgin zones is largest in layer III (see Table 3.4, $L_{m}$ contrasts of $28.20 \mathrm{~cm}$ ). Furthermore, the bottom sector logs in panel f show that $\phi_{N-L I R}$ in comparison to $\phi_{N-M C}$ yields the largest discrepancy, as much as $10 \mathrm{pu}$, in the invaded layer III. Figures 3.15 and 3.16 describe 3D FSFs at logging depths 
in bottom sector orientation across gas-filled layers II and III, respectively. The top row panels describe complex formation geometries in the vicinity of the tool caused by well deviation and invasion. Qualitative observations of 1D FSF radial and vertical projections in panels $\mathrm{c}, \mathrm{d}, \mathrm{g}$, and $\mathrm{h}$ show that $F S F_{L I R}$ does not accurately reproduce the spatial fluxes, while $F S F_{D F D}$ yields a better agreement with $F S F_{M C}$ despite the complex geometry.

\subsection{COMPUTATIONAL SPEED}

Rapid numerical simulation of nuclear logs is crucial for the development of advanced petrophysical interpretation methods that invoke quantitative integration with other logs (e.g. resistivity, sonic, and magnetic resonance). The interpretation of nuclear measurements has not kept pace with the interpretation of resistivity logs mainly because the MCNP code has traditionally been used for their numerical simulation. Albeit accurate and flexible, MCNP is computationally expensive, hence not suitable for inversion-based interpretation methods.

Table 3.5 compares the computational speeds of MCNP, LIR, and DFD for the numerical simulations of neutron porosity measurements discussed in this chapter; LIR is two times slower than DFD because it interpolates within the FSF library to obtain the most representative detector response of the formation model. The degree of variability and complexity in the formation tends to increase the number of iterations in the FSF refinement. For the cases discussed in this chapter, simulations were performed with a minimum and maximum of two and three iterations, respectively. The DFD perturbation method, summarized in workflow of Figure 3.5, does not require iterations in its numerical implementation, thus the improved computational speed compared to LIR. For adequate comparison of CPU time in Table 3.5, the computational time required to 
generate the base-case library set for DFD and LIR methods was subtracted from MCNP CPU times.

\subsection{Discussion}

Tool standoff has a greater effect on neutron logs than on density logs acquired at the bottom of the borehole in LWD environments. For example, density porosity could increase by $1 \mathrm{pu}$, while neutron porosity could increase by as much as $6 \mathrm{pu}$ in shales and $1 \mathrm{pu}$ in gas-bearing zones. Such a behavior can be attributed to (1) nonlinearity between formation property and neutron response, and (2) wider azimuthal aperture of neutron sensitivity in comparison to density sensitivity. For these reasons, the LIR as applied to $\phi_{N}$ simulation is not accurate to quantify flux perturbations due to tool standoff, thereby resulting in larger $\phi_{N}$ errors than with the DFD. These errors, proportional to standoff size (Appendix A), are especially severe at low porosities and across gas-filled formations because of large borehole-formation contrasts.

As described in Appendix A, the observed false gas crossover across layer IV in panel f of Figure 3.11 could be accentuated from 15 pu to $22 \mathrm{pu}$ when layer TSTs are decreased to $0.49 \mathrm{ft}(15 \mathrm{~cm})$. In such situations of severe shoulder-bed effects, LIR does not accurately reproduce the spatial distribution of perturbed FSFs, thereby resulting in $\phi_{N-L I R}$ errors as large as $5 \mathrm{pu}$.

The LIR requires pre-calculations of FSF libraries that include different borehole sizes and sector-binning orientations. A typical library for LIR includes MC-derived FSFs for 3 borehole sizes, 16 sector-binning orientations, 3 lithologies (sandstone, limestone, and dolomite), and the varying formation porosities described in Table 3.2. On the other hand, the DFD method does not require extensive library pre-calculations because perturbed FSFs can be rapidly and accurately calculated upon demand; each 
base-case DFD function can be re-used for any perturbation magnitude, variation, or/and lithology (Figure 3.5). This implementation reduces computational time and memory requirements for the library by a factor of 144 because FSFs are calculated only for those formation base-cases described in Table 3.2. Similarly, in the benchmark invasion case documented in Figure 3.10, only three base-case FSFs were used to perform calculations for all 25 invasion fronts. This important property further decreases both computational time and memory requirements.

Accuracy and robustness of forward models are the most important factors for efficient inversion applications. The FSF- $F_{p}$ FFM, discussed in Chapter 2, is valid for formations saturated with various fluid constituents, while the DFD method can simulate measurements acquired under complex geometrical and physical conditions. Even though the two methods in this first part of the dissertation exhibit significant degrees of robustness, the DFD technique is better than the FSF- $F_{p}$ FFM for the following reasons:

(1) From the described cases, the DFD method is accurate to less than 1 pu while the FSF- $F_{p}$ FFM maintains a 2 pu tolerance.

(2) Note that the FSF- $F_{p}$ FFM still implements the LIR so that the DFD technique is two times faster than the FSF- $F_{p}$ FFM.

(3) The DFD technique implements explicit neutron physics, i.e., a one-group diffusion model, to calculate lumped higher-order perturbation terms, while the $F_{p}$ is an empirically calculated parameter for implementation of a first-order calculation with the LIR.

(4) It follows from the DFD method that spatial perturbations in neutron FSFs can be accounted for in the inversion of neutron porosity measurements. This is not possible with the FSF- $F_{p}$ FFM. 


\subsection{CONCLUSIONS}

I developed and validated a new transport-diffusion method based on fluxdifference approximations, i.e., Rytov DFD, for rapid and efficient simulation of neutron porosity measurements acquired with wireline and LWD tools. The new DFD method performs 3D spatial flux perturbations and implements a lumped higher-order perturbation calculation for simulating neutron porosity measurements. Empirical transport-correction coefficients implemented with the simulation method remain constant per detector and per tool across rocks with various solid compositions and fluid constituents.

The new DFD method is more accurate than linear iterative refinement (LIR) for neutron porosity modeling in the presence of neutron cross-section perturbations caused by shoulder-bed effects, standoff, mud-filtrate invasion, and formation dip. Simulations indicate that the accuracy of neutron porosities calculated with the DFD method is approximately $1 \mathrm{pu}$ while errors associated with LIR can be as high as $10 \mathrm{pu}$ at bed boundaries in $\mathrm{HA} / \mathrm{HZ}$ wells. It was found that the DFD method is two times faster than LIR in most of the simulation cases documented in this dissertation.

Calculation of perturbed detector sensitivity functions is a crucial step in the numerical simulation of nuclear measurements. Qualitative comparisons against MCNP of FSFs obtained with the DFD method and LIR showed that DFD is more accurate, reliable, and efficient than LIR for calculating detector sensitivity fluxes. Another important step in nuclear porosity modeling is accurate perturbation calculations. Firstorder perturbation for neutron porosity simulations is accurate in simple formation geometries with minimal variability in rock properties, e.g., vertical wells penetrating horizontal beds. The magnitude of flux perturbations increases with formation variability (e.g., HA wells penetrating gas-saturated formations with borehole environmental 
effects), to the extent that higher-order perturbation terms dominate the calculations. In these situations, LIR porosity errors could be as large as $10 \mathrm{pu}$. The DFD circumvents such a problem by implementing a lumped higher-order perturbation model; associated errors in simulated neutron porosity are drastically reduced to below $1 \mathrm{pu}$ for complex formation geometries, even in extreme cases of tool standoff, invasion, shoulder-bed effects, and HA wells.

The availability of rapid, accurate, and efficient algorithms, such as the DFD method, to simulate borehole nuclear measurements opens the possibility of developing rapid inversion-based petrophysical and compositional interpretation methods in conjunction with resistivity and magnetic resonance logs. It was found that the DFD method is more efficient and accurate than the FSF- $F_{p}$ FFM for applications of inversionbased interpretation. Chapter 4 discusses inversion application of the DFD technique. 
Table 3.1: Transport-correction coefficients, $\eta$, calculated for LWD and wireline tools, per detector.

\begin{tabular}{|c|c|c|}
\hline & $\eta\left(\mathbf{r}_{\mathrm{R}}\right)=\eta_{\mathrm{far}}$ & $\eta\left(\mathbf{r}_{\mathrm{R}}\right)=\eta_{\text {near }}$ \\
\hline $\begin{array}{c}\text { LWD neutron tool, with DT } \\
\text { (14 MeV) source }\end{array}$ & 1.062 & 0.8736 \\
\hline $\begin{array}{c}\text { Wireline neutron tool, with } \\
\text { AmBe source }\end{array}$ & 1.614 & 1.129 \\
\hline
\end{tabular}


Table 3.2: Summary of homogeneous formation base-cases and their neutron porosity $\log$ responses in limestone porosity units (pu).

\begin{tabular}{|c|c|c|c|c|c|c|}
\hline \multirow[t]{2}{*}{$\begin{array}{c}\text { Base-case } \\
\text { description }\end{array}$} & \multirow{2}{*}{$\begin{array}{c}\text { Volumetric } \\
\text { concentrations } \\
(\mathrm{v} / \mathrm{v})\end{array}$} & \multirow{2}{*}{$\begin{array}{c}\text { Bulk } \\
\text { density, } \\
\rho_{b} \\
\left(\mathrm{~g} / \mathrm{cm}^{3}\right)\end{array}$} & \multicolumn{2}{|c|}{$\begin{array}{c}\text { Migration } \\
\text { length, } L_{m}(\mathrm{~cm})\end{array}$} & \multicolumn{2}{|c|}{$\begin{array}{c}\text { Neutron } \\
\text { porosity, } \\
\phi_{N} \\
\text { (limestone pu) }\end{array}$} \\
\hline & & & $\begin{array}{c}\text { Wireline } \\
\text { tool }\end{array}$ & $\begin{array}{l}\text { LWD } \\
\text { tool }\end{array}$ & $\begin{array}{l}\text { Wireline } \\
\text { tool }\end{array}$ & $\begin{array}{c}\text { LWD } \\
\text { tool }\end{array}$ \\
\hline LIME00 & $\begin{array}{l}\text { Limestone block: } \\
1.00 \mathrm{CaCO}_{3}\end{array}$ & 2.7100 & 28.34 & 29.31 & 0.30 & -0.18 \\
\hline LIME05W & $\begin{array}{c}0.05 \mathrm{H}_{2} \mathrm{O} \\
0.95 \mathrm{CaCO}_{3}\end{array}$ & 2.6245 & 20.72 & 22.08 & 4.34 & 5.55 \\
\hline LIME10W & $\begin{array}{c}0.10 \mathrm{H}_{2} \mathrm{O} \\
0.90 \mathrm{CaCO}_{3}\end{array}$ & 2.5390 & 17.54 & 19.18 & 9.02 & 9.62 \\
\hline LIME20W & $\begin{array}{c}0.20 \mathrm{H}_{2} \mathrm{O} \\
0.80 \mathrm{CaCO}_{3}\end{array}$ & 2.3680 & 14.13 & 16.24 & 19.11 & 19.55 \\
\hline LIME30W & $\begin{array}{c}0.30 \mathrm{H}_{2} \mathrm{O} \\
0.70 \mathrm{CaCO}_{3}\end{array}$ & 2.1970 & 12.20 & 14.73 & 30.62 & 31.02 \\
\hline LIME40W & $\begin{array}{c}0.40 \mathrm{H}_{2} \mathrm{O} \\
0.60 \mathrm{CaCO}_{3}\end{array}$ & 2.0260 & 10.93 & 13.85 & 40.66 & 39.44 \\
\hline LIME20G & $\begin{array}{c}0.20 \mathrm{CH}_{4} \\
\left(0.0178 \mathrm{~g} / \mathrm{cm}^{3}\right) \\
0.80 \mathrm{CaCO}_{3}\end{array}$ & 2.1716 & 32.05 & 33.37 & -0.73 & -1.72 \\
\hline LIME30G & $\begin{array}{c}0.30 \mathrm{CH}_{4} \\
\left(0.0178 \mathrm{~g} / \mathrm{cm}^{3}\right) \\
0.70 \mathrm{CaCO}_{3}\end{array}$ & 1.9023 & 34.60 & 36.18 & -1.09 & -2.49 \\
\hline LIME40G & $\begin{array}{c}0.40 \mathrm{CH}_{4} \\
\left(0.0178 \mathrm{~g} / \mathrm{cm}^{3}\right) \\
0.60 \mathrm{CaCO}_{3}\end{array}$ & 1.6331 & 37.83 & 39.78 & -1.38 & -3.22 \\
\hline
\end{tabular}


Table 3.3: Summary of assumed properties for the synthetic layered earth model.

\begin{tabular}{|c|c|c|c|c|}
\hline Layer & $\begin{array}{c}\text { Volumetric } \\
\text { concentrations (v/v) }\end{array}$ & $\begin{array}{c}\text { Migration } \\
\text { length LWD, } \\
\boldsymbol{L}_{\boldsymbol{m}}(\mathbf{c m})\end{array}$ & $\begin{array}{c}\text { Migration } \\
\text { length } \\
\text { WIRELINE, } \\
\boldsymbol{L}_{\boldsymbol{m}}(\mathbf{c m})\end{array}$ & $\begin{array}{c}\text { Bulk } \\
\text { density, } \boldsymbol{\rho}_{\boldsymbol{b}} \\
\left(\mathbf{g} / \mathbf{c m}^{\mathbf{3}}\right)\end{array}$ \\
\hline I & $\begin{array}{c}0.05 \mathrm{H}_{2} \mathrm{O} \\
0.40 \mathrm{SiO}_{2} \\
0.50 \mathrm{Al}_{2} \mathrm{Si}_{2} \mathrm{O}_{5}(\mathrm{OH})_{4} \\
0.05 \mathrm{FeS}_{2}\end{array}$ & 14.72 & 12.74 & 2.6655 \\
\hline II & $\begin{array}{c}0.05 \mathrm{CH}_{4}\left(0.0178 \mathrm{~g} / \mathrm{cm}^{3}\right) \\
0.95 \mathrm{SiO}_{2}\end{array}$ & 37.02 & 35.98 & 2.5184 \\
\hline III & $\begin{array}{c}0.30 \mathrm{CH}_{4}\left(0.0178 \mathrm{~g} / \mathrm{cm}^{3}\right) \\
0.70 \mathrm{SiO}_{2}\end{array}$ & 43.10 & 41.5 & 1.8603 \\
\hline IV & $\begin{array}{c}0.30 \mathrm{H}_{2} \mathrm{O} \\
0.70 \mathrm{SiO}_{2}\end{array}$ & 15.60 & 25.03 & 2.1550 \\
\hline V & $\begin{array}{c}0.05 \mathrm{H}_{2} \mathrm{O} \\
0.95 \mathrm{SiO}_{2}\end{array}$ & 25.44 & 24.07 & 2.5675 \\
\hline
\end{tabular}


Table 3.4: Summary of assumed properties for the approximate "teardrop" invasion profile in the synthetic earth model.

\begin{tabular}{|c|c|c|c|c|c|c|c|}
\hline \multirow{2}{*}{ Layer } & \multicolumn{2}{|c|}{$\begin{array}{c}\text { Approximate } \\
\text { "teardrop" invasion } \\
\text { profile geometry }\end{array}$} & \multicolumn{2}{|c|}{$\begin{array}{c}\text { Migration length for } \\
\text { LWD tool, } L_{m}(\mathbf{c m})\end{array}$} & \multicolumn{2}{c|}{$\begin{array}{c}\text { Bulk density, } \\
\rho_{b}\left(\mathrm{~g} / \mathbf{c m}^{3}\right)\end{array}$} \\
\cline { 2 - 8 } & $\begin{array}{c}\text { invasion } \\
\text { radius } \\
\text { (in.) }\end{array}$ & $\begin{array}{c}\text { cylinder } \\
\text { eccentered } \\
\text { distance (in.) }\end{array}$ & $\begin{array}{c}\text { invaded } \\
\text { zone }\end{array}$ & $\begin{array}{c}\text { virgin } \\
\text { zone } \\
\text { contrast in } \\
\text { virgin and } \\
\text { invaded zones }\end{array}$ & $\begin{array}{c}\text { zone } \\
\text { invaded }\end{array}$ & $\begin{array}{c}\text { virgin } \\
\text { zone }\end{array}$ \\
\hline I & 6.30 & 1.71 & 14.23 & 14.72 & 0.49 & 2.5850 & 2.6655 \\
\hline II & 10.63 & 1.71 & 24.78 & 37.02 & 12.24 & 2.5575 & 2.5184 \\
\hline III & 7.87 & 1.71 & 14.90 & 43.10 & 28.20 & 2.0950 & 1.8603 \\
\hline IV & 7.87 & 1.71 & 14.90 & 15.60 & 0.70 & 2.0950 & 2.1550 \\
\hline V & 10.63 & 1.71 & 24.78 & 25.44 & 0.66 & 2.5575 & 2.5675 \\
\hline
\end{tabular}


Table 3.5: Summarized CPU times, quantified in the numerical simulation of neutron porosity measurements, of benchmark and LWD HA/HZ examples using MCNP, LIR, and DFD methods.

\begin{tabular}{|c|c|c|c|c|}
\hline & & $\begin{array}{c}\text { Using a Linux } \\
\text { cluster with Intel } \\
\text { Xeon 6-core } 2.93\end{array}$ & \multicolumn{2}{|c|}{$\begin{array}{l}\text { Using a Windows XP PC with } \\
\text { dual-core 3.2 GHz processors }\end{array}$} \\
\hline & & MCNP & LIR & DFD \\
\hline \multirow{2}{*}{$\begin{array}{l}\text { CPU } \\
\text { time }\end{array}$} & $\begin{array}{l}\text { Benchmark examples, } \\
\text { per sample point }\end{array}$ & 5870 seconds & 0.172 seconds & 0.0801 seconds \\
\hline & $\begin{array}{l}\text { LWD HA/HZ examples, per } \\
\text { depth, for a complete tool } \\
\text { rotation of } 16 \text { azimuthal bins }\end{array}$ & $\begin{array}{l}1460 \text { minutes } \\
(\sim 24 \text { hours })\end{array}$ & $\begin{array}{l}0.0450 \text { minutes } \\
(\sim 2.7 \text { seconds })\end{array}$ & $\begin{array}{l}0.0230 \text { minutes } \\
(\sim 1.4 \text { seconds })\end{array}$ \\
\hline
\end{tabular}


(a)

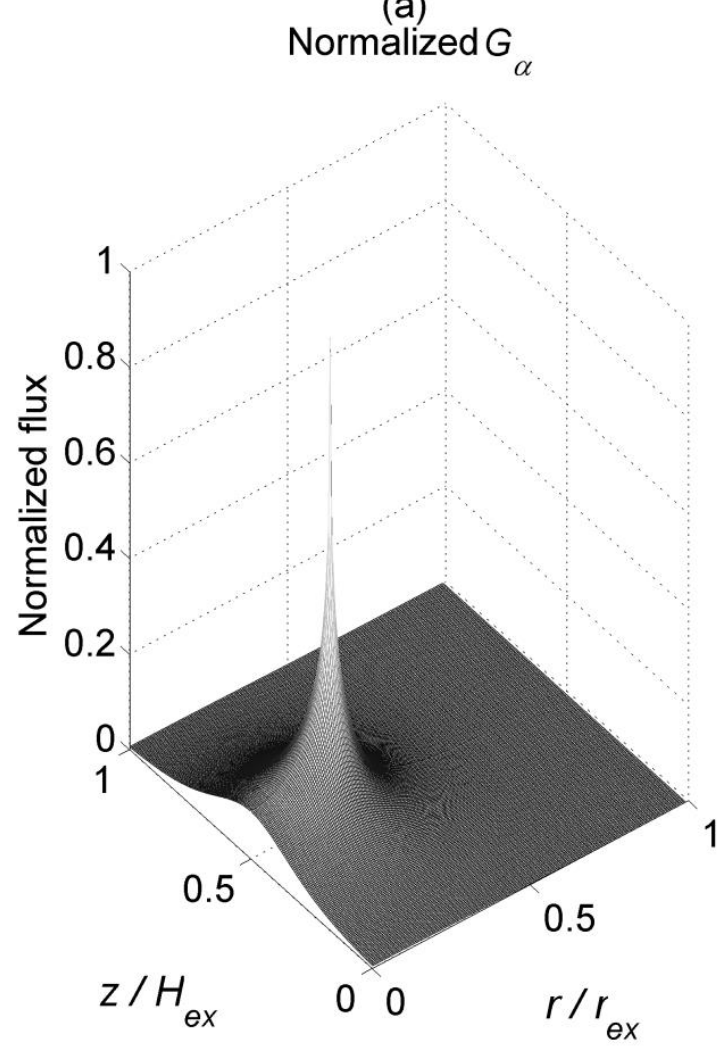

(b)

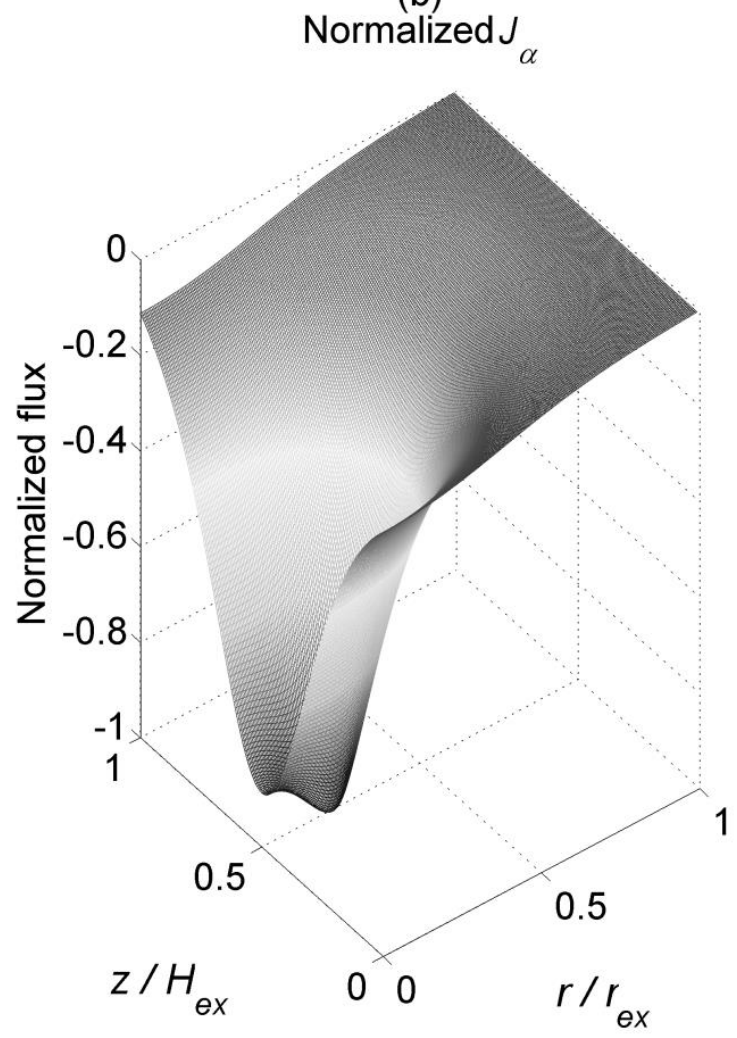

Figure 3.1: $\quad$ Normalized 2D diffusion kernels of neutron point source located at $\mathbf{r}_{S}=\mathbf{r}_{S}\left(r_{S}, z_{S}\right)=\left(0.25 r_{e x}, 0.5 H_{e x}\right)$ for $\alpha_{B}^{-1}=30 \mathrm{~cm}$, using equations 3.3 and 3.4. (a) Green's function kernel, $G_{\alpha}$ and (b) its derivative, $J_{\alpha}$. 


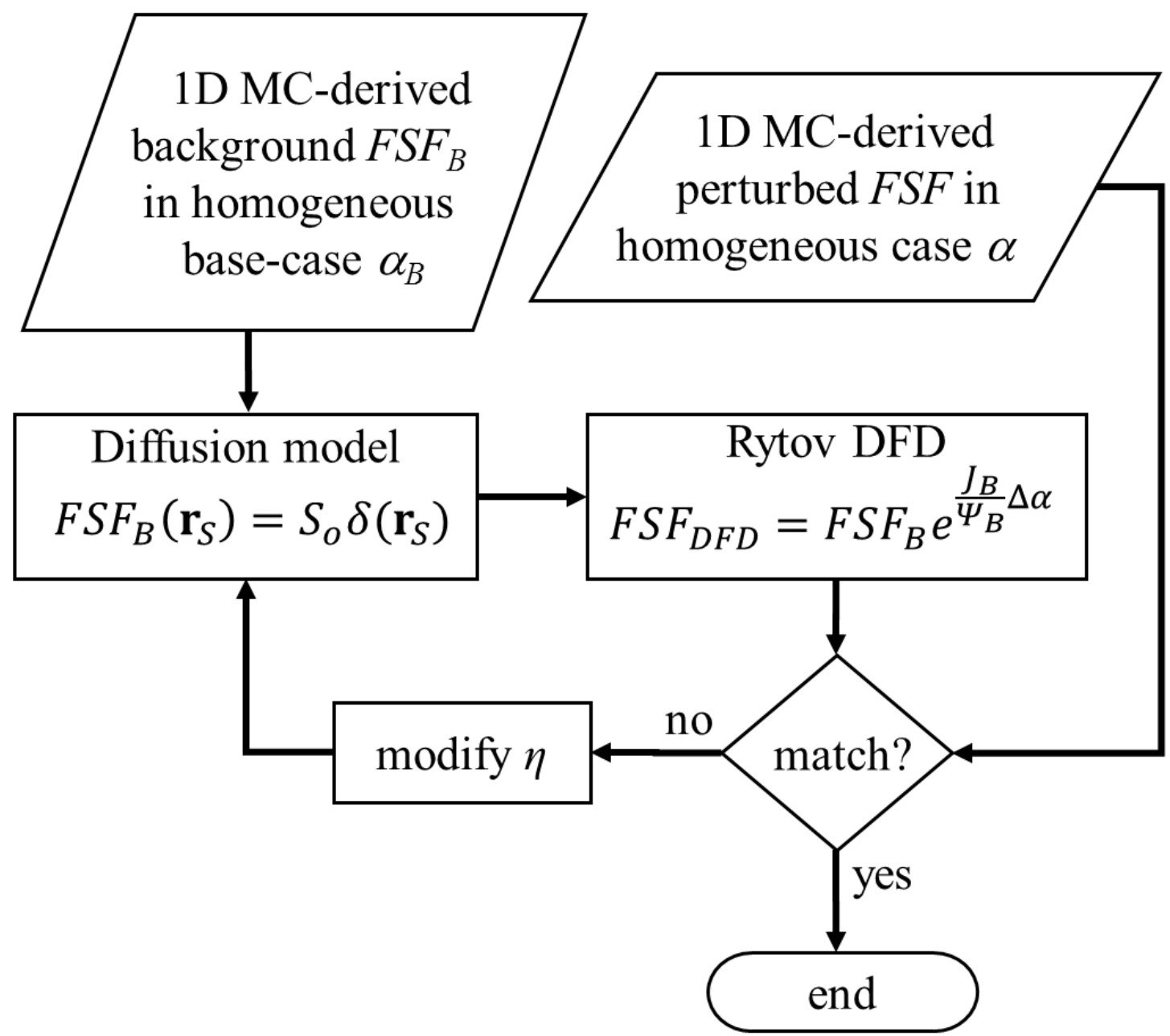

Figure 3.2: Flow diagram of the flux-fitting method used to calculate transportcorrection coefficient, $\eta$. 
(a) LWD tool with DT source

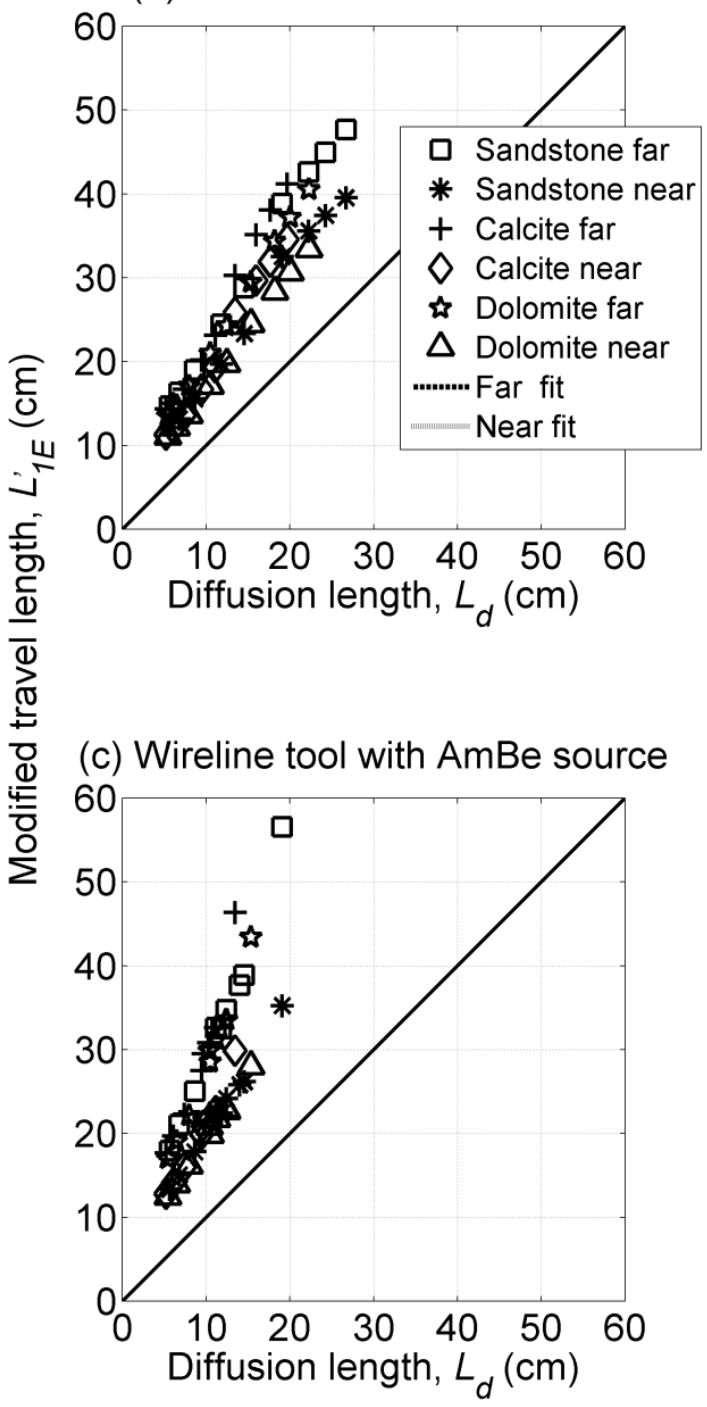

(b) LWD tool with DT source

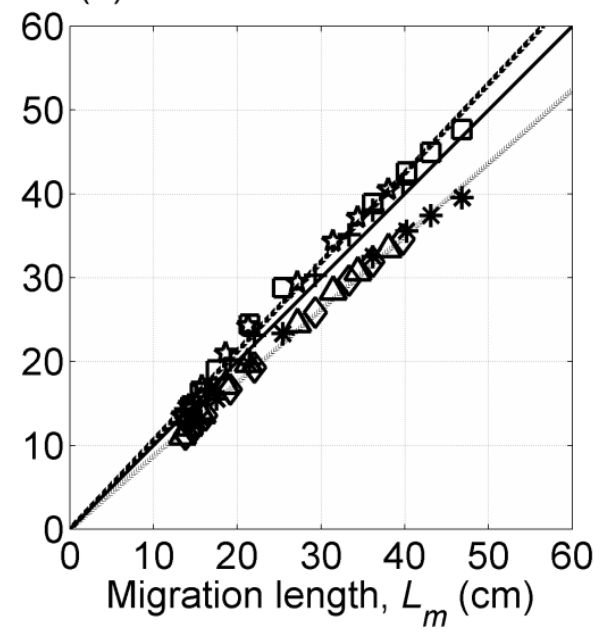

(d) Wireline tool with AmBe source

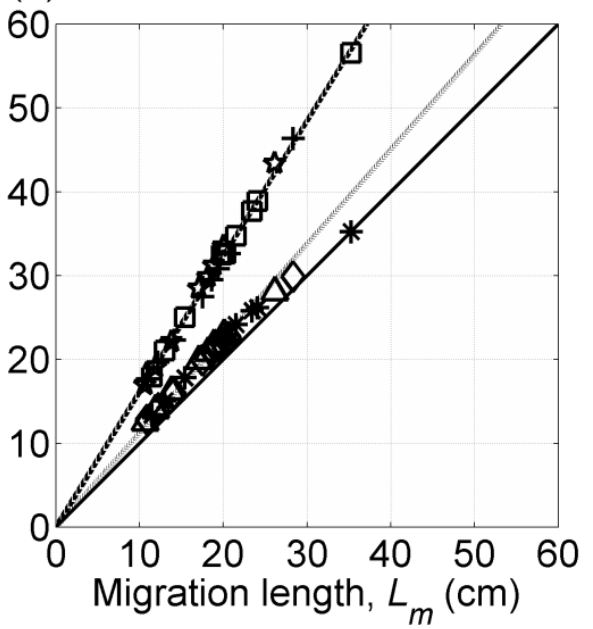

Figure 3.3: Calculation of the transport-correction coefficient, $\eta$ across various basecases of water- and gas-filled lithologies. (a) Cross-plot of modified travel length, $L^{\prime}{ }_{1 E}$ and diffusion length, $L_{d}$. (b) Cross-plot of modified travel length, $L^{\prime}{ }_{1 E}$ and migration length, $L_{m}$ for an LWD neutron tool with a 14 MeV DT source. Slopes of solid black, dashed black, and dotted gray lines identify unity, $\eta$ of far detector, and $\eta$ of near detector, respectively. (c)(d) Similar to (a) and (b), respectively, but for a wireline tool with an AmBe neutron source. 

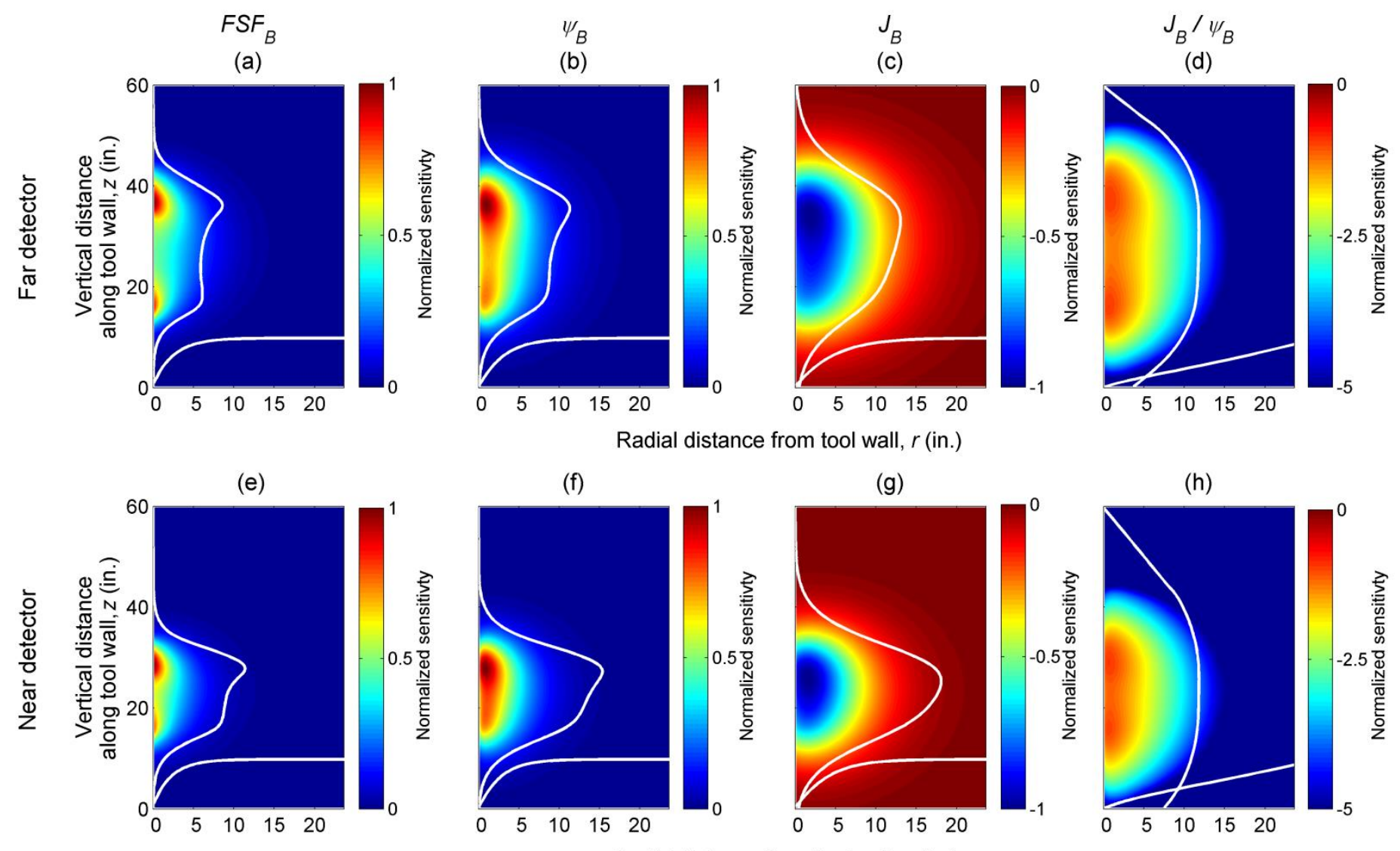

(g)

(h)
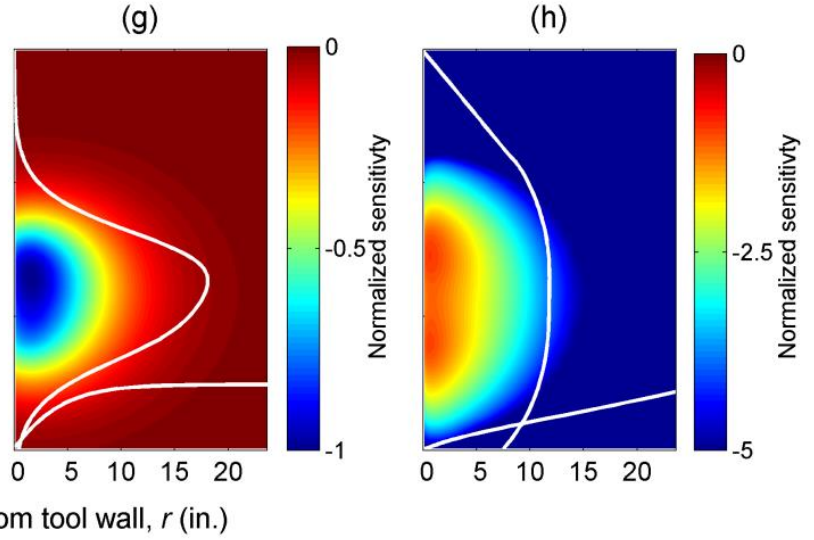

Figure 3.4: Description of FSF and DFD spatial distribution functions for the LWD neutron tool across a limestone block. Top and bottom panels describe far and near detector responses, respectively. (a)(e) Monte Carlo-derived $F S F_{B}$, (b)(f) forward diffusion distributed function, $\psi_{B},(\mathrm{c})(\mathrm{g})$ derivative diffusion distributed function, $J_{B}$, and (d)(h) Rytov DFD function, $J_{B} / \psi_{B}$. Colors describe the relative 2D spatial sensitivity. Solid white lines describe the $1 \mathrm{D}$ vertical projection and radial $\mathrm{J}$-factor of relative spatial sensitivities. 


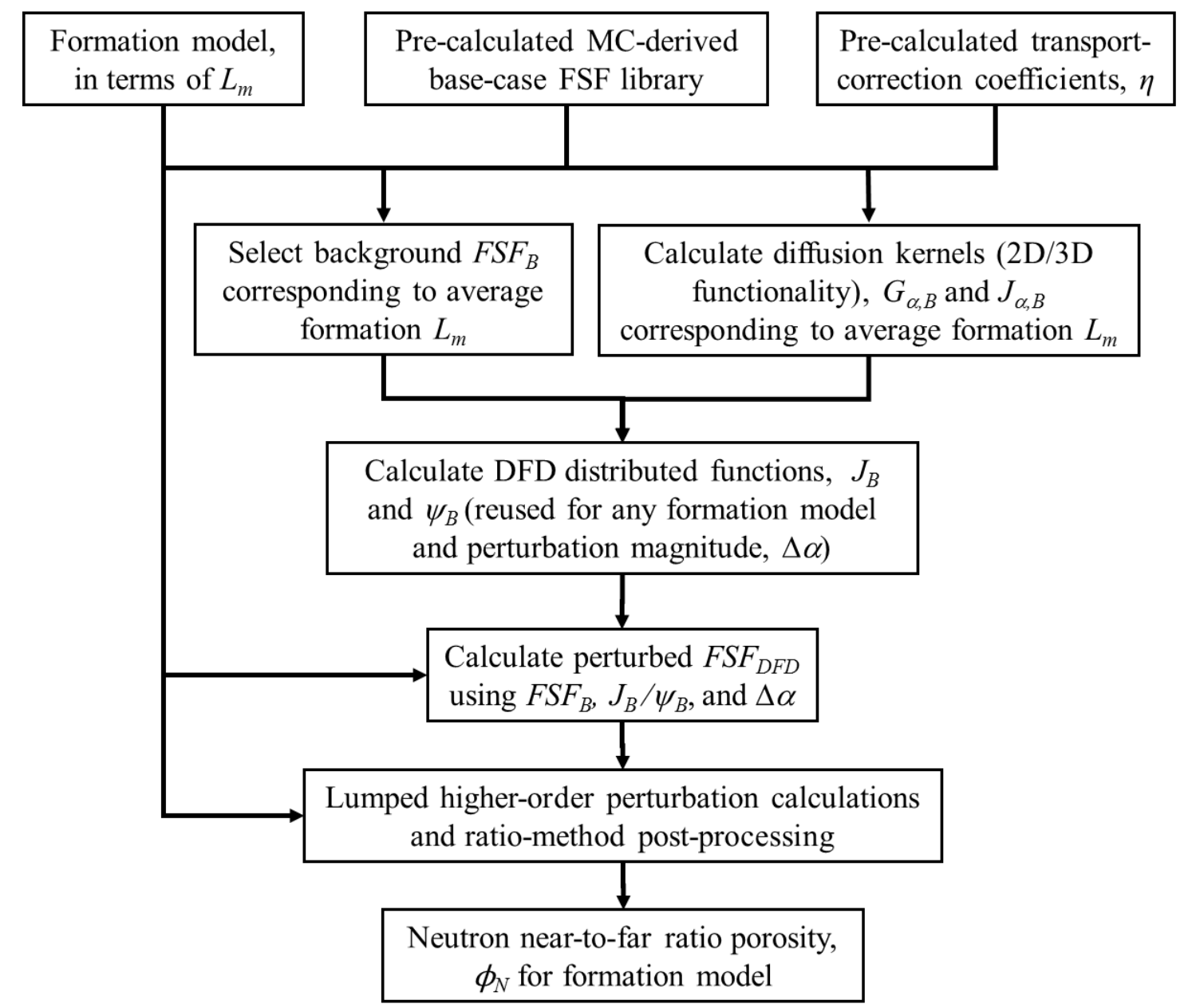

Figure 3.5: Flow diagram describing the algorithm and implementation of the DFD model for neutron porosity, $\phi_{N}$, simulation. 
(a)

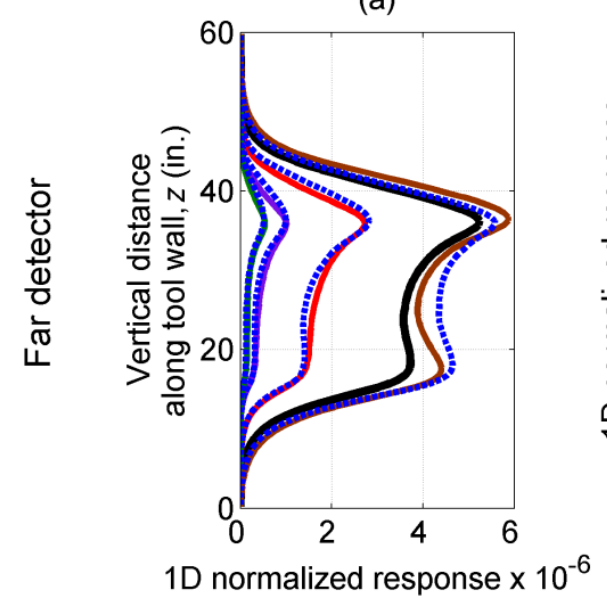

(c)

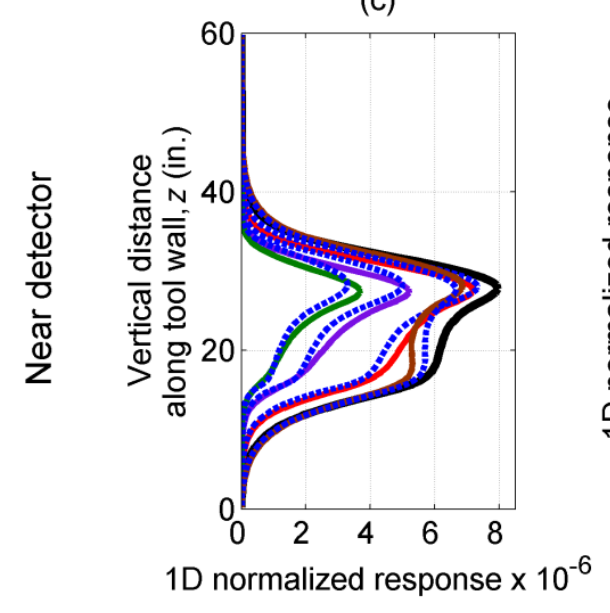

(b)
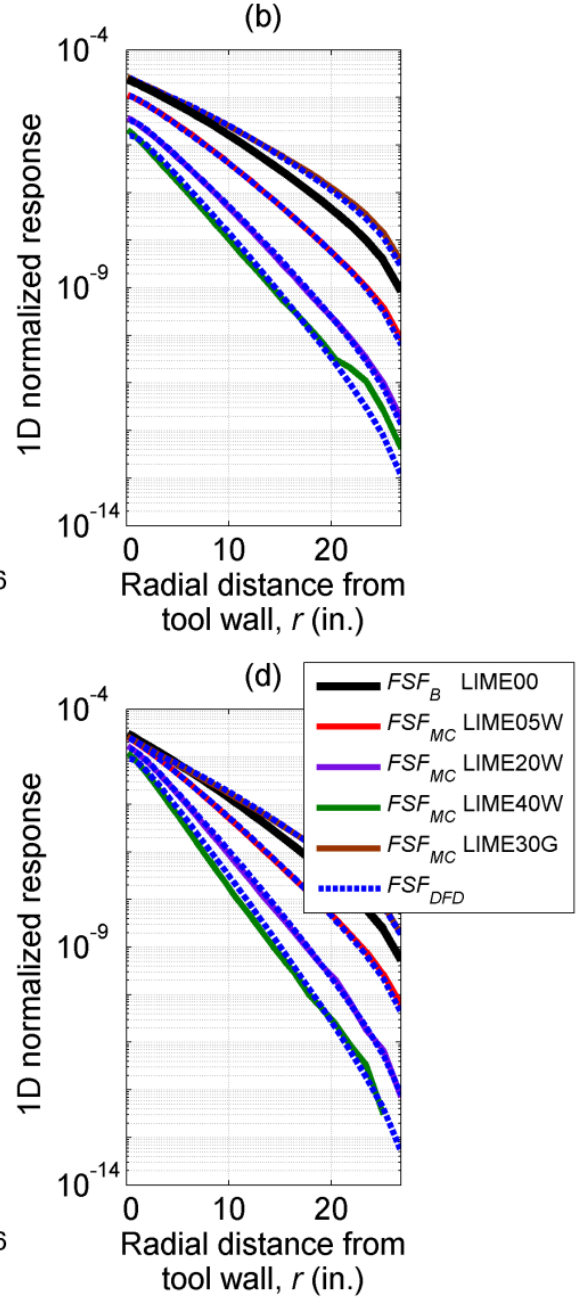

Figure 3.6: Qualitative comparisons of the DFD method to full MCNP calculations across water- and gas-filled limestone formations. Left and right panels show 1D FSF vertical and radial projections, respectively. In this case, the 2D kernel functionality is used for DFD calculations of the LWD tool. (a) Far detector FSF vertical projections, integrated in the radial and azimuthal directions, showing background (limestone block) MC-derived $F S F_{B}$ with a solid black line, MC-derived perturbed $F S F_{M C}$ in homogeneous water- and gas-filled limestone base-cases in solid colored lines, and corresponding DFD perturbed $F S F_{D F D}$ with a dashed blue line. (b) Far detector FSF radial projections, integrated in the vertical and azimuthal directions. (c) Similar to (a) but for the near detector. (d) Similar to (b) but for the near detector. 
(a)
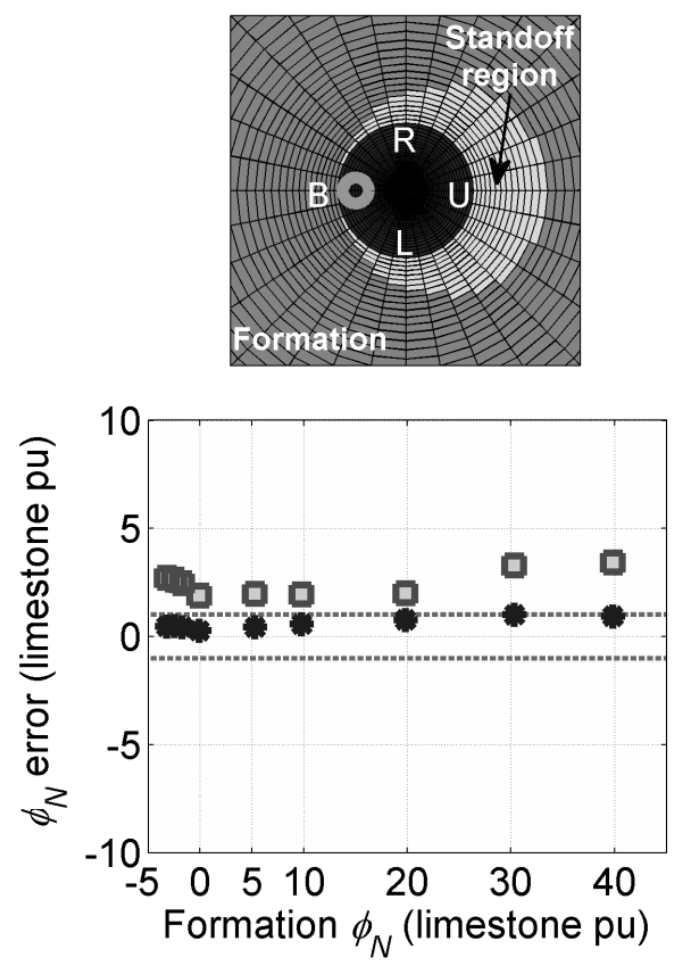

(b)
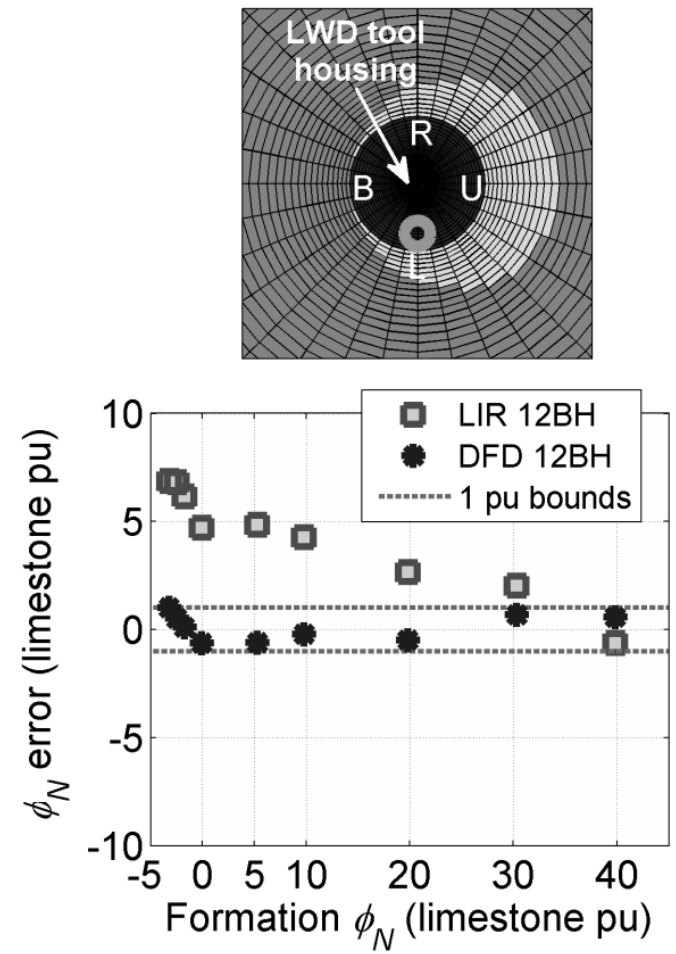

(c)
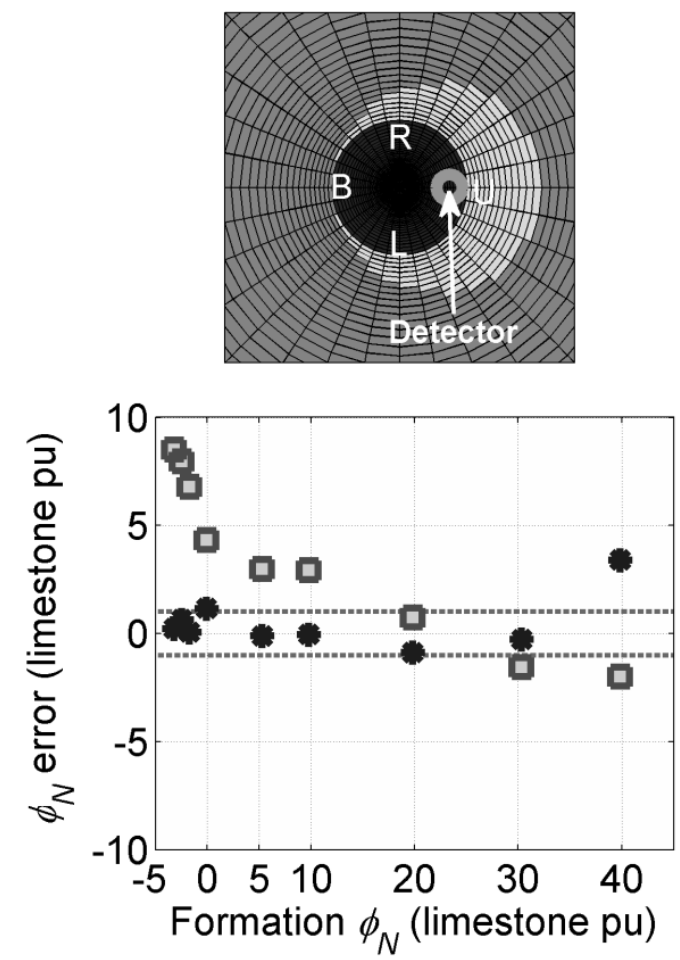

Figure 3.7: Quantitative comparisons of 8.5-in-collar LWD $\phi_{N}$ calculated with DFD and LIR methods against MCNP at (a) bottom, B sector orientation, (b) left, L sector orientation, and (c) up, U sector orientation, in a 12-in. borehole, across various formation base-cases. The schematic diagram above each panel describes the corresponding tool orientation in the borehole. DFD calculations were performed by invoking their 3D kernel functionality. The borehole is assumed to be filled with fresh water. 

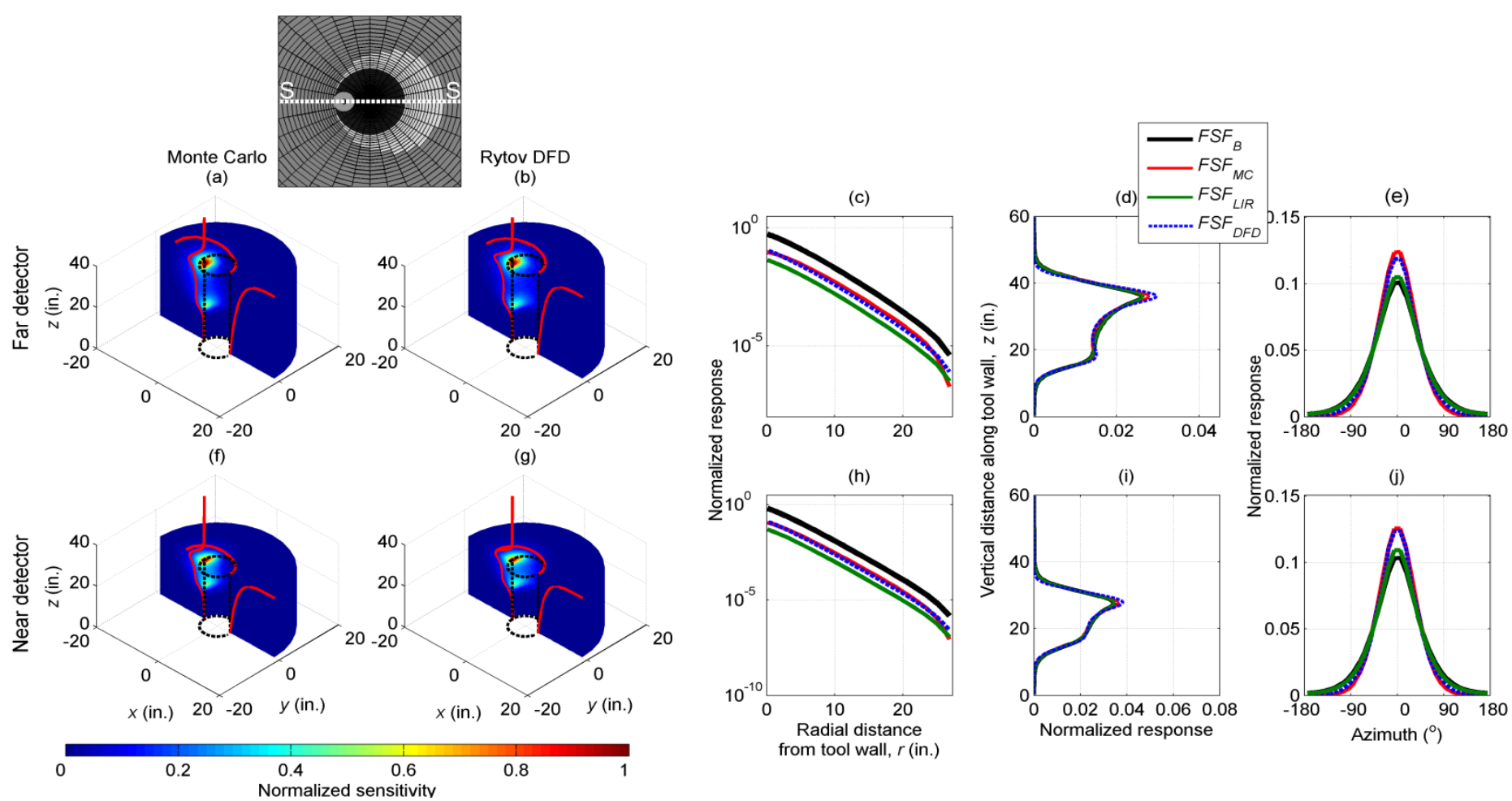

Figure 3.8: Qualitative comparisons of 3D FSFs calculated with MCNP, DFD and LIR methods of LWD detector responses at bottom, B sector orientation across a $5 \%$, water-filled limestone formation. The borehole is assumed filled with fresh water. Top and bottom row panels describe FSFs of far and near detectors, respectively. (a)(f) Perturbed detector FSFs using full MCNP calculations, $F S F_{M C}$ and (b)(g) perturbed detector FSFs using the DFD method, $F S F_{D F D}$ viewed at the cross-section $S-S$ ' shown in the borehole schematic describing the corresponding tool orientation in the borehole; 1D FSF projections are identified with solid red lines, i.e., vertical projection, radial J-factor, and azimuthal projection in polar coordinates. (c)(h) 1D FSF radial projections, (d)(i) 1D FSF vertical projections, and (e)(j) 1D FSF azimuthal projections; DFD calculations were performed by invoking their 3D kernel functionality. 

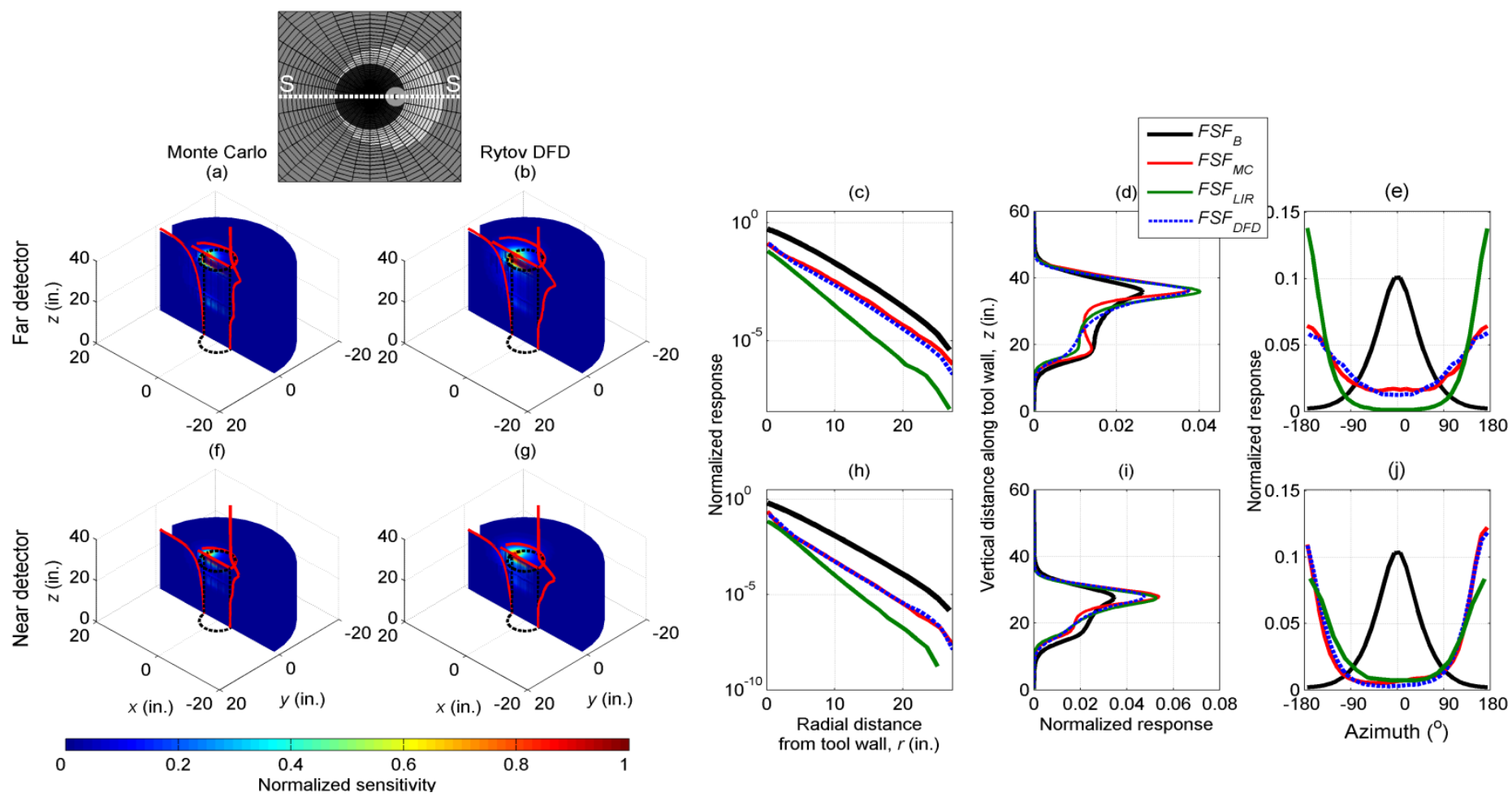

Figure 3.9: Qualitative comparisons of 3D FSFs calculated with MCNP, DFD and LIR methods of LWD detector responses at up, U sector orientation across a $5 \%$, water-filled limestone formation. The borehole is assumed filled with fresh water. Top and bottom row panels describe FSFs of far and near detectors, respectively. (a)(f) Perturbed detector FSFs using full MCNP calculations, $F S F_{M C}$ and (b)(g) perturbed detector FSFs using the DFD method, $F S F_{D F D}$ viewed at the cross-section $S-S$ ' shown in the borehole schematic describing the corresponding tool orientation in the borehole; 1D FSF projections are identified with solid red lines, i.e., vertical projection, radial J-factor, and azimuthal projection in polar coordinates. (c)(h) 1D FSF radial projections, (d)(i) 1D FSF vertical projections, and (e)(j) 1D FSF azimuthal projections; DFD calculations were performed by invoking their 3D kernel functionality. 

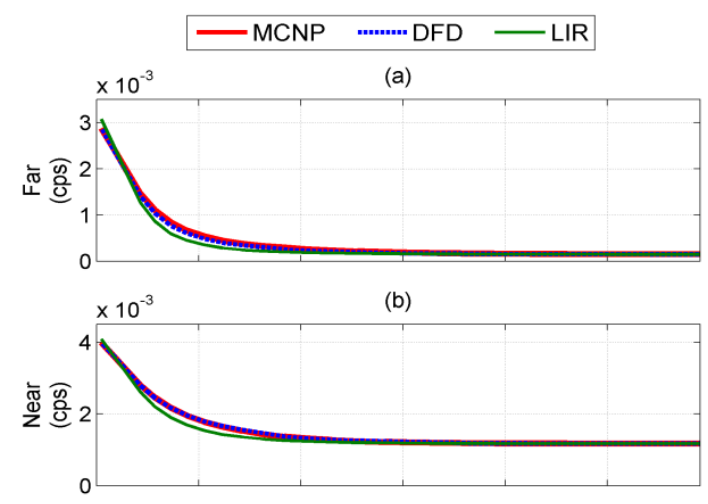

(c)

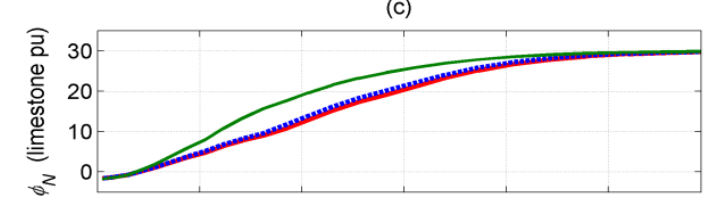

(d)
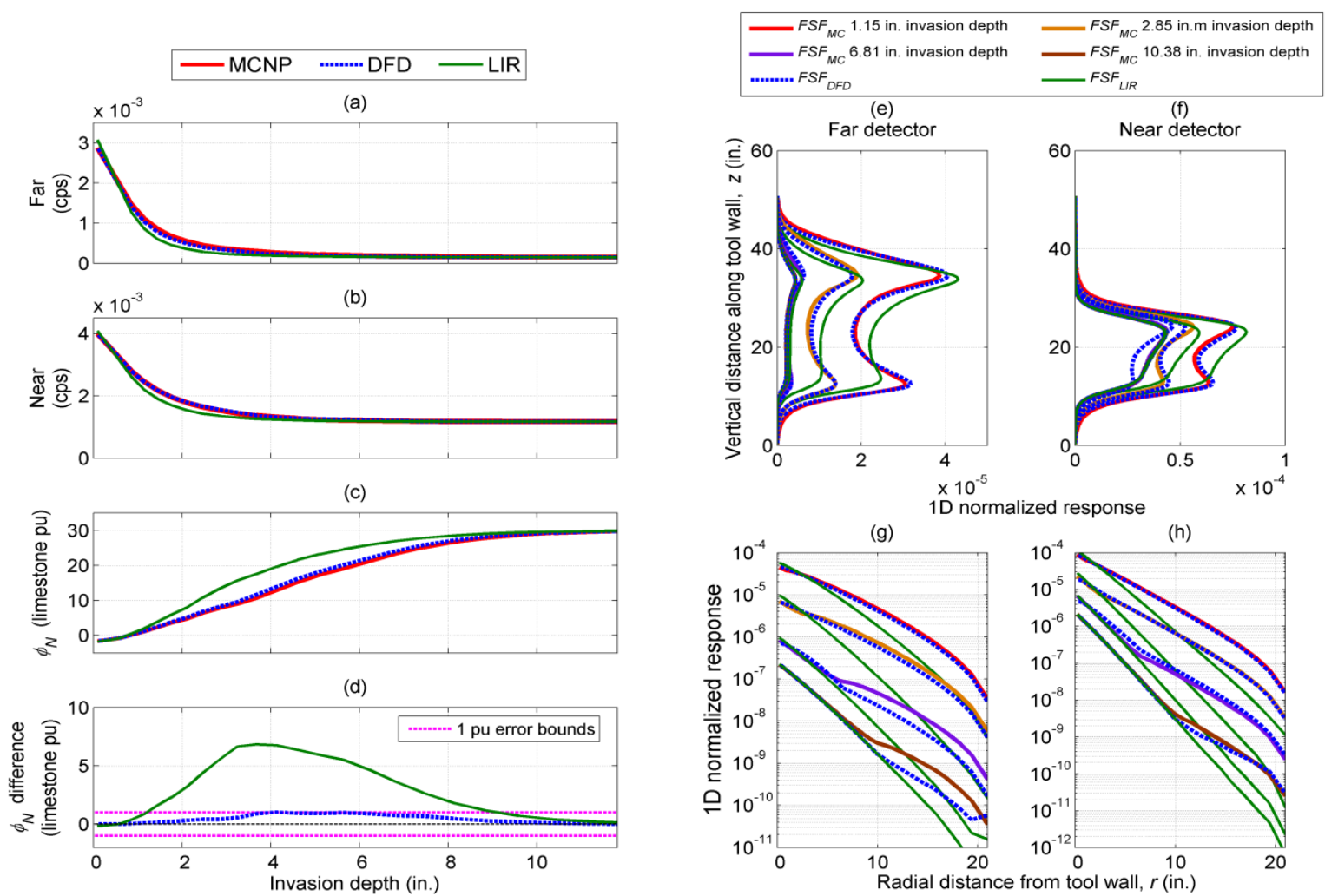

Figure 3.10: Comparisons of MCNP, DFD and LIR methods in the presence of fresh water invasion of a gas-filled $30 \%$ limestone formation. Quantitative comparisons of $\phi_{N}$ measurements are shown in the first column, while qualitative comparisons of FSFs are shown in the second and third columns. (a) Simulated far detector counts, (b) simulated near detector counts, (c) simulated $\phi_{N}$ measurements in limestone pu, and (d) $\phi_{N}$ differences versus invasion depth. MCNP, DFD, and LIR measurements are identified with solid red, dashed blue, and solid green lines, respectively. (e) $1 \mathrm{D}$ (vertical) far detector FSFs, showing $F S F_{M C}, F S F_{D F D}$, and $F S F_{L I R}$ at various invasion fronts from the borehole wall. (f) Similar to (e) but for the near detector. (g) 1D (radial) far detector FSFs, and (h) 1D (radial) near detector FSFs. The borehole is assumed to be filled with fresh water. 
(a) Formation model in $L_{m}$
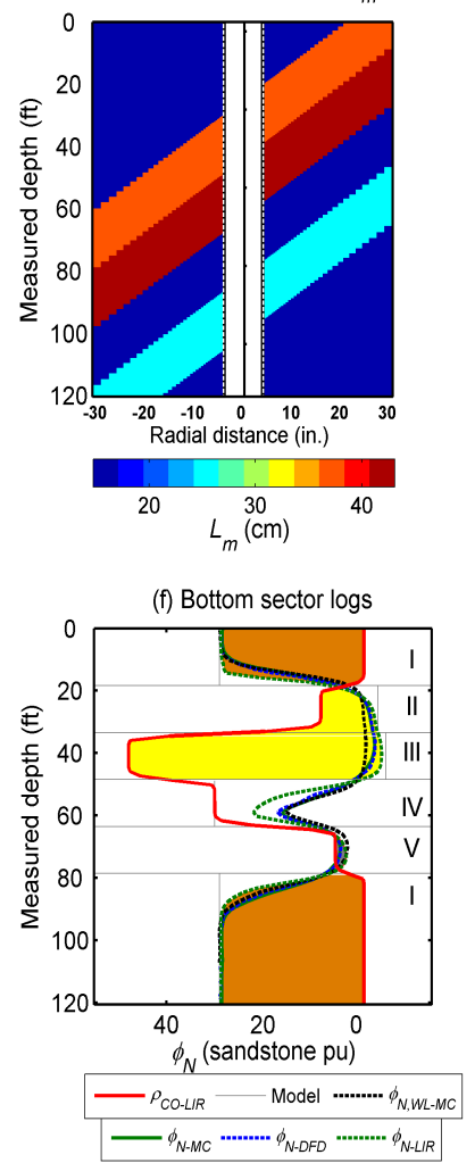

(b) $\phi_{N-L I R}$

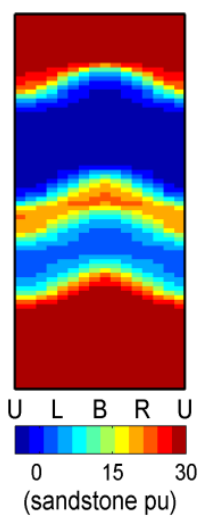

(g) $\phi_{N-M C}-\phi_{N-L I R}$

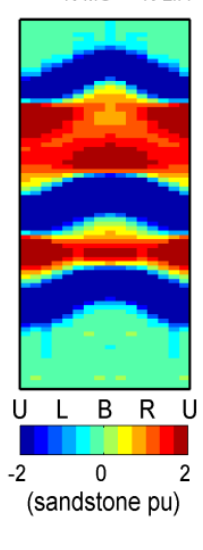

(c) $\phi_{N-D F D}$

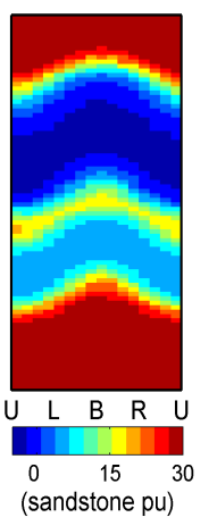

(h) $\phi_{N-M C}-\phi_{N-D F D}$

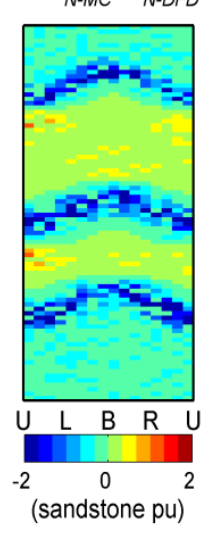

(d) $\phi_{N-M C}$

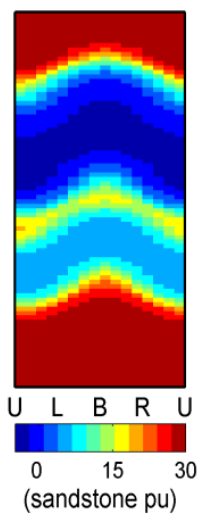

(i) Average error

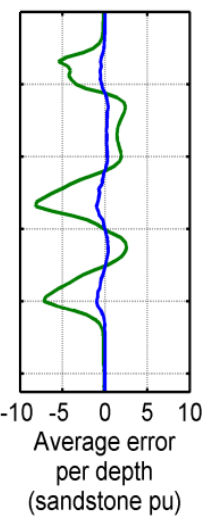

(e) $\rho_{\text {CO-LIR }}$

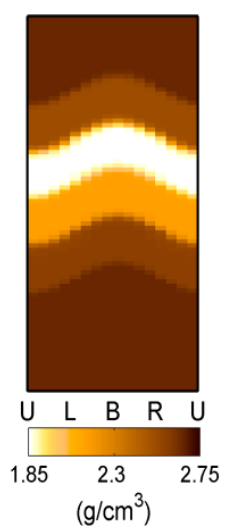

ลิ (j) Average error

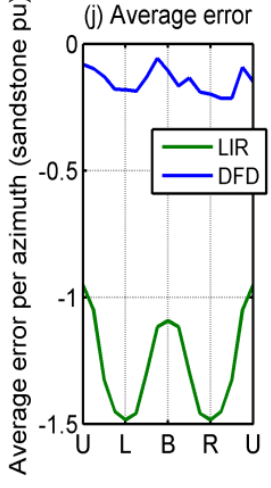

Figure 3.11: Comparisons of LWD neutron porosities $\phi_{N-M C}, \phi_{N-D F D}$, and $\phi_{N-L I R}$, in sandstone pu, simulated with MCNP, DFD, and LIR methods, respectively, in a slick borehole for the synthetic model described in Table 3.3 where TST is $1.3 \mathrm{ft}$. (a) Description of the layered earth model in $L_{m}$ (not to scale), (b) neutron porosity, $\phi_{N-L I R}$ calculated with the LIR method, (c) neutron porosity, $\phi_{N-D F D}$ calculated with the DFD method, (d) neutron porosity, $\phi_{N-M C}$ rendered with full MCNP calculations, (e) compensated density $\rho_{C O-L I R}$ calculated with LIR, (f) bottom sector neutron and density logs (layer descriptions included in Table 3.3), (g) LWD porosity residuals, $\phi_{N-M C}-\phi_{N-L I R}$, (h) LWD porosity residuals, $\phi_{N-M C}-\phi_{N-D F D}$, (i) average error per depth, and (j) average error per azimuth; DFD calculations were performed by invoking their 3D kernel functionality. 

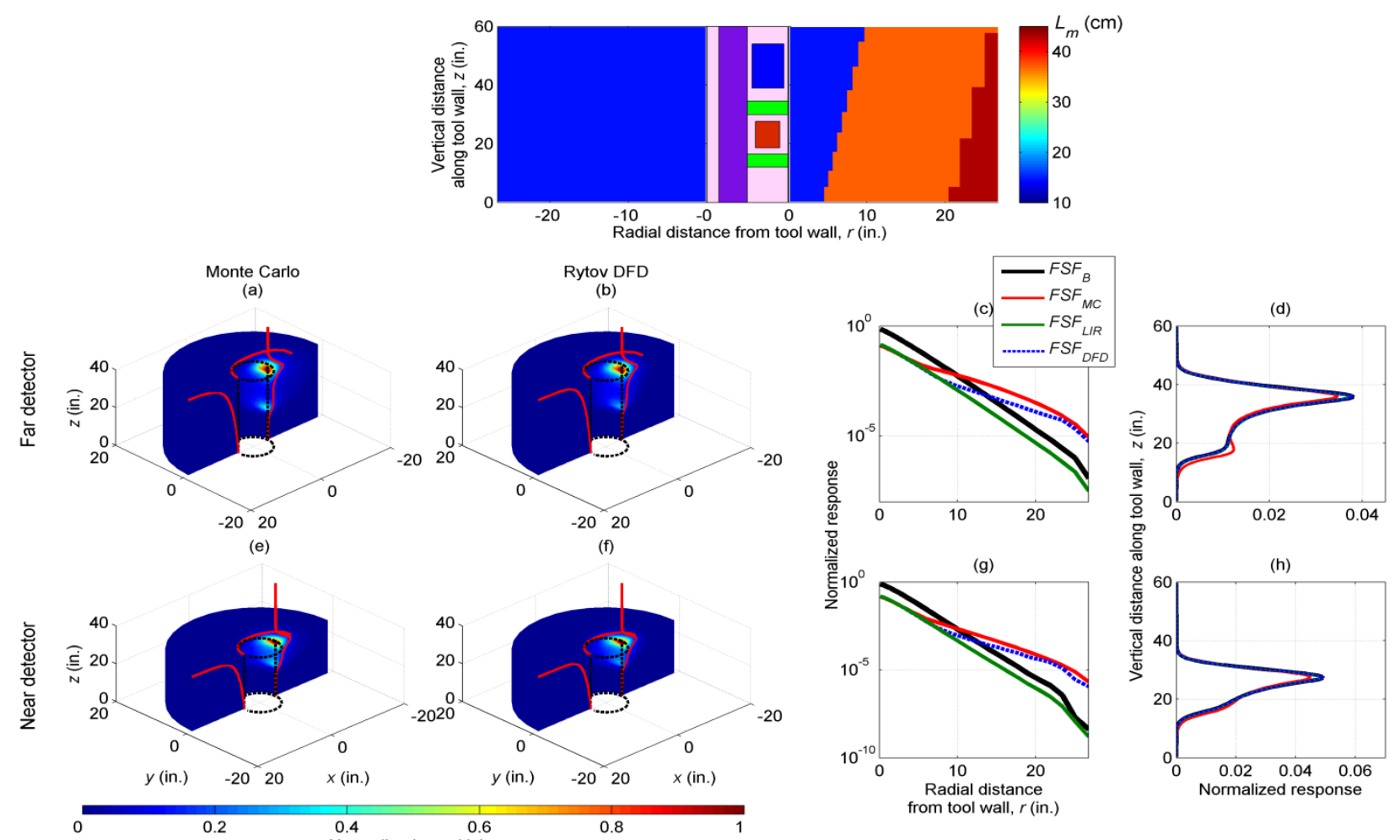

Figure 3.12: Qualitative comparisons of LWD bottom, B sector FSFs at $15.8 \mathrm{ft}(4.81 \mathrm{~m})$ measured depth across the layered earth model described in Table 3.3. The top row panel describes the LWD tool and layered earth model in $L_{m}$ (not to scale) at this sample depth. Middle and bottom row panels describe FSFs for far and near detectors, respectively. (a)(e) Perturbed detector FSFs rendered with full MCNP calculations, $F S F_{M C}$, (b)(f) perturbed detector FSFs calculated with the DFD method, $F S F_{D F D}$; 1D FSF projections are identified with solid red lines, i.e., vertical projection, radial J-factor, and azimuthal projection in polar coordinates. (c)(g) 1D FSF radial projections, and (d)(h) 1D FSF vertical projections; DFD calculations were performed by invoking their 3D kernel functionality. 

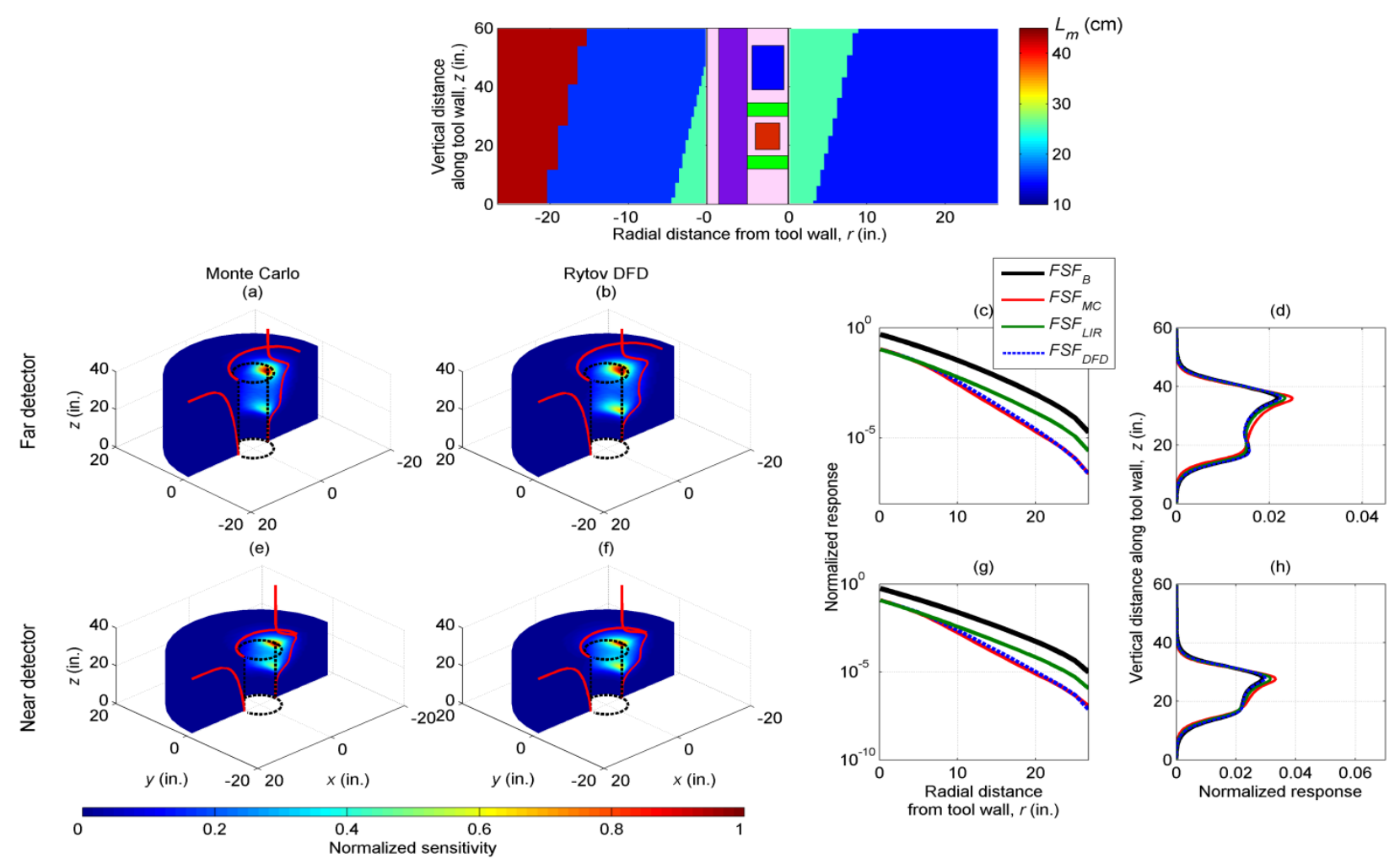

Figure 3.13: Qualitative comparisons of LWD bottom, B sector FSFs at $76.0 \mathrm{ft}(23.2 \mathrm{~m})$ measured depth, across the layered earth model described in Table 3.3. The top row panel describes the LWD tool and layered earth model in $L_{m}$ (not to scale) at this sample depth. Middle and bottom row panels describe FSFs for far and near detectors, respectively. (a)(e) Perturbed detector FSFs rendered with full MCNP calculations, $F S F_{M C}$, (b)(f) perturbed detector FSFs calculated with the DFD method, $F S F_{D F D}$; 1D FSF projections are identified with solid red lines, i.e., vertical projection, radial J-factor, and azimuthal projection in polar coordinates. (c)(g) 1D FSF radial projections, and (d)(h) 1D FSF vertical projections; DFD calculations were performed by invoking their 3D kernel functionality. 
(a) Formation model in $L_{m}$
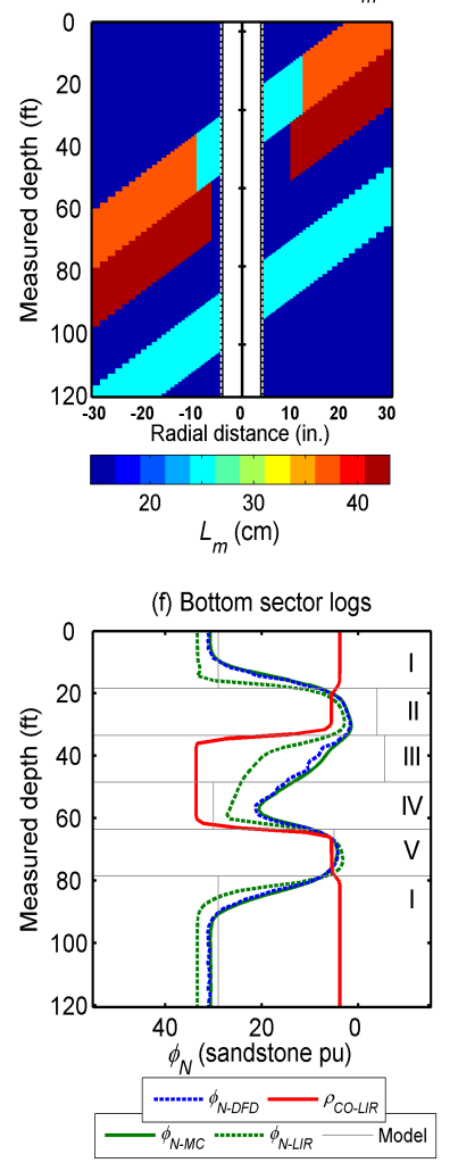

(b) $\phi_{N-L I R}$

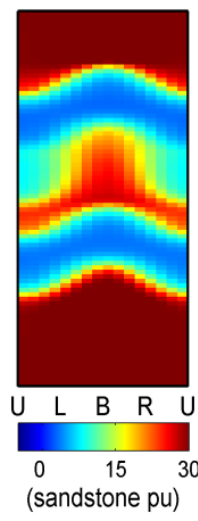

(g) $\phi_{N-M C}-\phi_{N-L I R}$

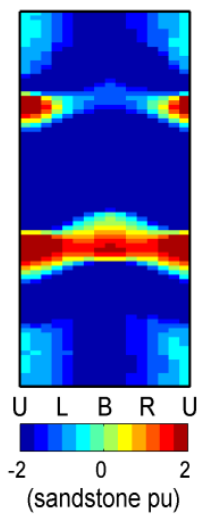

(c) $\phi_{N-D F D}$

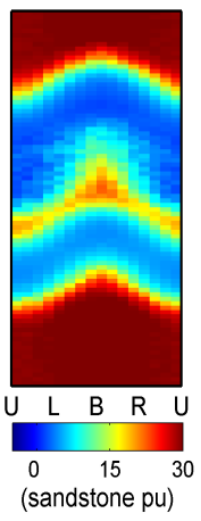

(h) $\phi_{N-M C}-\phi_{N-D F D}$

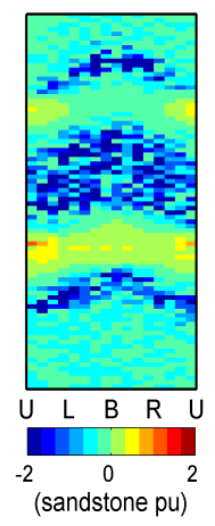

(d) $\phi_{N-M C}$

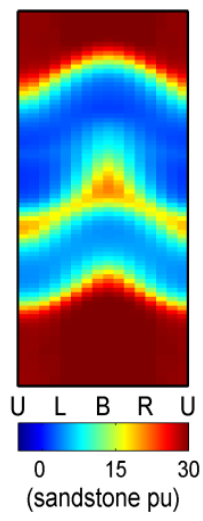

(i) Average error

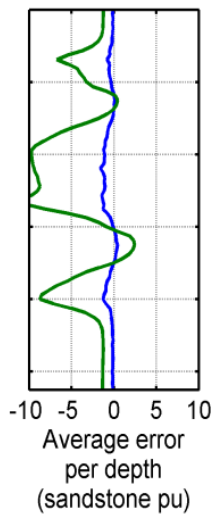

(e) $\rho_{\text {CO-LIR }}$
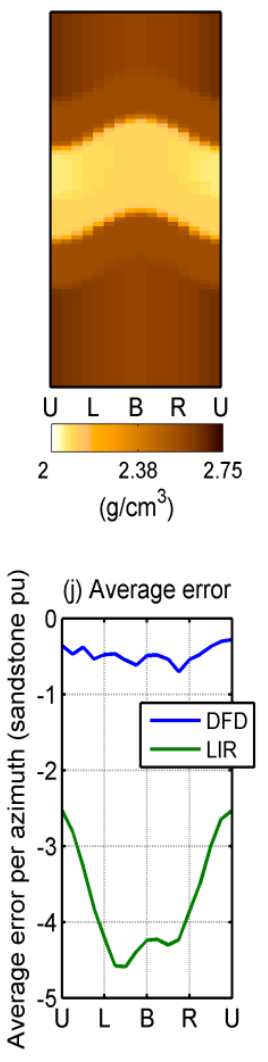

Figure 3.14: Comparisons of LWD neutron porosities $\phi_{N-M C}, \phi_{N-D F D}$, and $\phi_{N-L I R}$, in sandstone pu, calculated with MCNP, DFD, and LIR methods, respectively, in a slick borehole for the invaded synthetic model described in Table 3.4 where TST is $1.3 \mathrm{ft}$. (a) Description of the layered earth model in $L_{m}$ (not to scale), (b) neutron porosity, $\phi_{N-L I R}$ calculated with the LIR method, (c) neutron porosity, $\phi_{N-D F D}$ calculated with the DFD method, (d) neutron porosity, $\phi_{N-M C}$ rendered with full MCNP calculations, (e) compensated density $\rho_{C O-L I R}$ calculated with LIR, (f) bottom sector neutron and density logs (layer descriptions included in Table 3.4), (g) LWD porosity residuals, $\phi_{N-M C}-\phi_{N-L I R}$, (h) LWD porosity residuals, $\phi_{N-M C}-\phi_{N-}$ $D F D$, (i) average error per depth, and (j) average error per azimuth; DFD calculations were performed by invoking their 3D kernel functionality. 

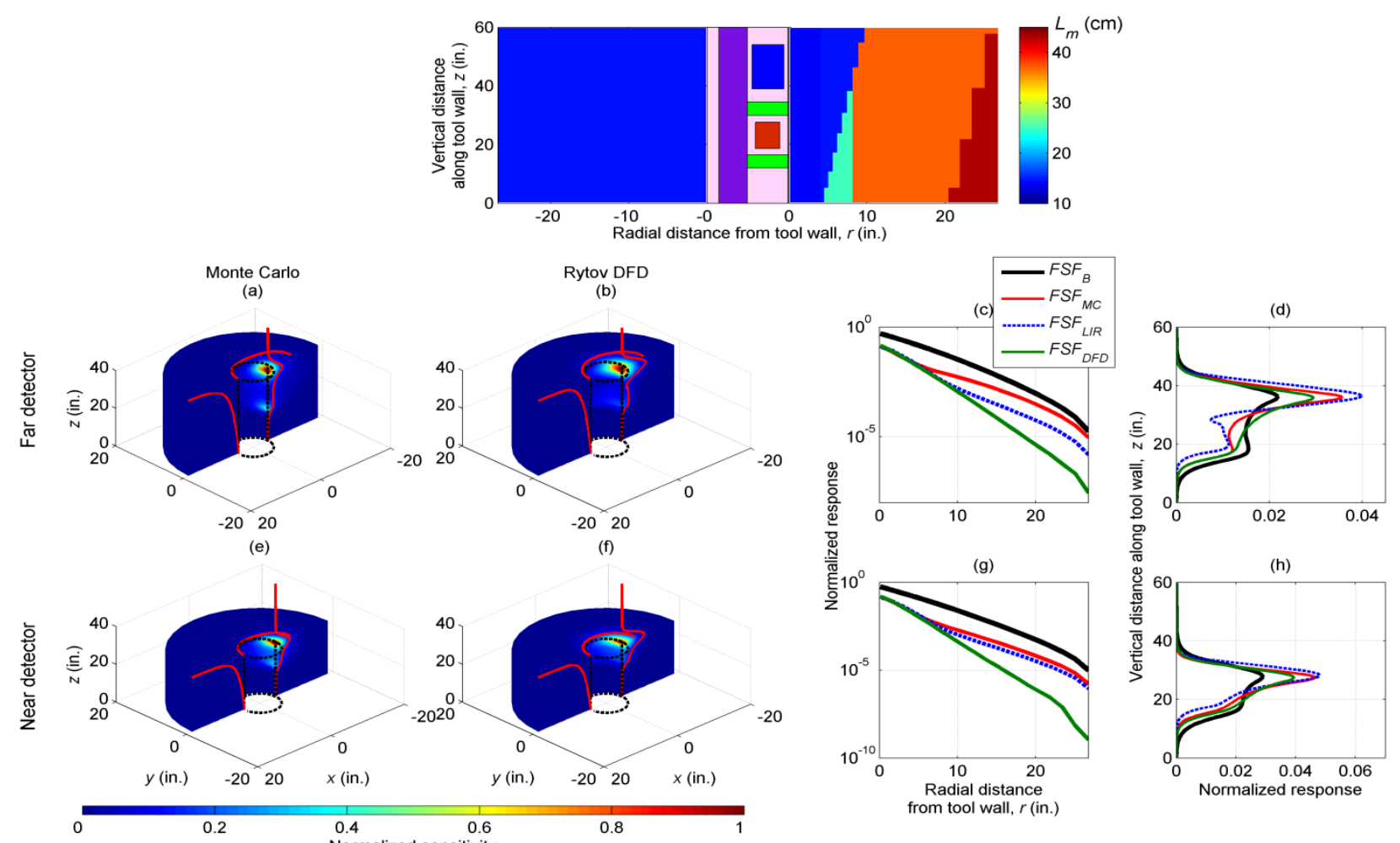

Figure 3.15: Qualitative comparisons of LWD bottom, B sector FSFs at $15.8 \mathrm{ft}(4.81 \mathrm{~m})$ measured depth, across the invaded layered earth model described in Table 3.4. The top row panel describes the LWD tool and layered earth model in $L_{m}$ (not to scale) at this sample depth. Middle and bottom row panels describe FSFs for far and near detectors, respectively. (a)(e) Perturbed detector FSFs rendered with full MCNP calculations, $F S F_{M C}$, (b)(f) perturbed detector FSFs calculated with the DFD method, $F S F_{D F D}$; 1D FSF projections are identified with solid red lines, i.e., vertical projection, radial J-factor, and azimuthal projection in polar coordinates. (c)(g) 1D FSF radial projections, and (d)(h) 1D FSF vertical projections; DFD calculations were performed by invoking their 3D kernel functionality. 

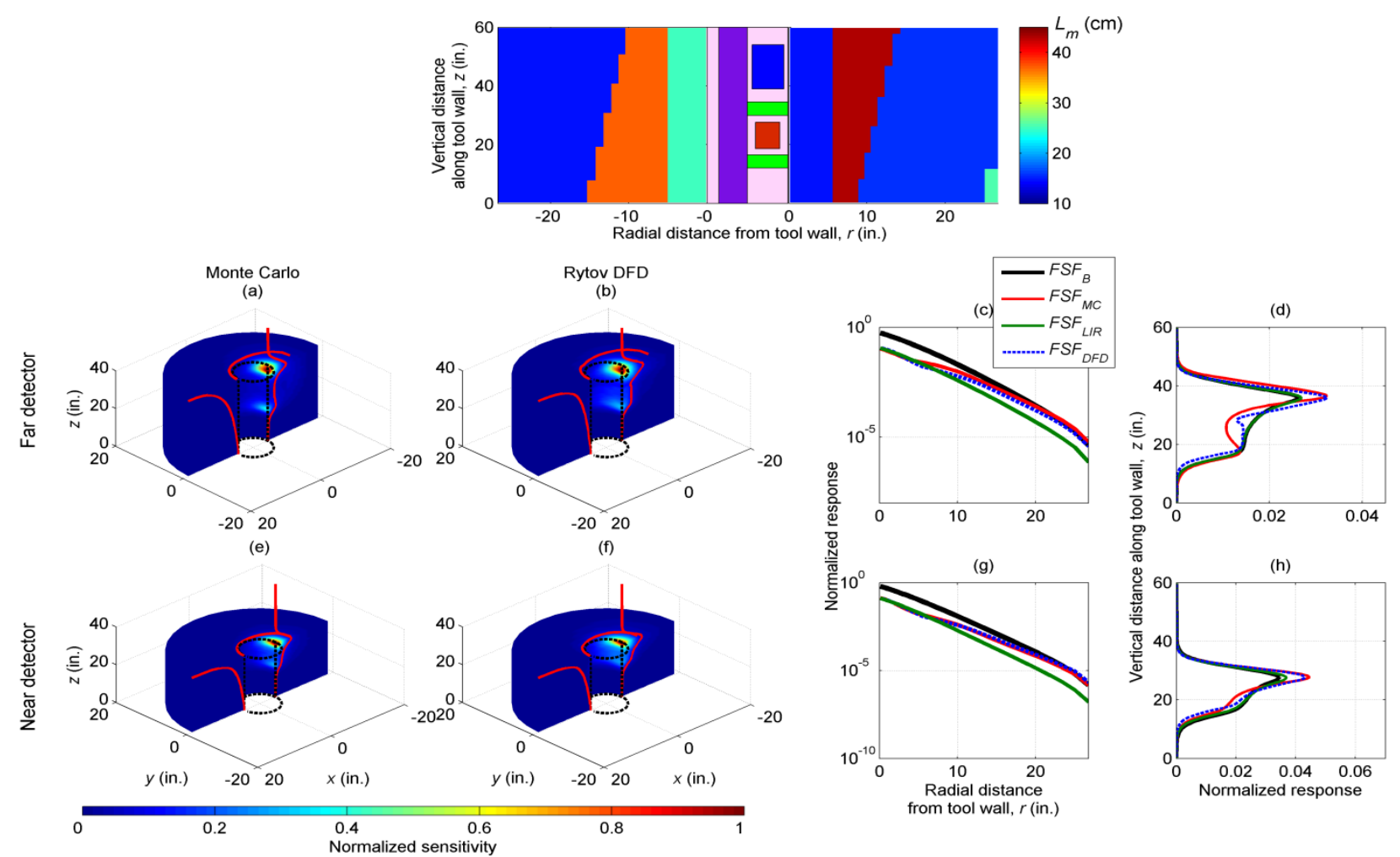

Figure 3.16: Qualitative comparisons of LWD bottom, B sector FSFs at $41.6 \mathrm{ft}(12.7 \mathrm{~m})$ measured depth, across the invaded layered earth model described in Table 3.4. The top row panel describes the LWD tool and layered earth model in $L_{m}$ (not to scale) at this sample depth. Middle and bottom row panels describe FSFs for far and near detectors, respectively. (a)(e) Perturbed detector FSFs rendered with full MCNP calculations, $F S F_{M C}$, (b)(f) perturbed detector FSFs calculated with the DFD method, $F S F_{D F D}$; 1D FSF projections are identified with solid red lines, i.e., vertical projection, radial J-factor, and azimuthal projection in polar coordinates. (c)(g) 1D FSF radial projections, and (d)(h) 1D FSF vertical projections; DFD calculations were performed by invoking their 3D kernel functionality. 


\section{Chapter 4: Inversion-Based Petrophysical Interpretation of Logging- While-Drilling Nuclear and Resistivity Measurements}

Interpretation of borehole measurements acquired in high-angle and horizontal (HA/HZ) wells is challenging due to significant influence of well trajectory and bed geometrical effects. Experience shows that accurate integrated interpretation of well logs acquired in $\mathrm{HA} / \mathrm{HZ}$ wells requires explicit consideration of the three-dimensional (3D) measurement physics. The most reliable alternative for interpretation of well logs in $\mathrm{HA} / \mathrm{HZ}$ wells is with inversion techniques that correct measurements for shoulder-bed, undulating well trajectory, and bed geometrical effects while taking advantage of high data resolution. I introduce an efficient layer-based inversion workflow for combined, quantitative petrophysical and compositional interpretation of logging-while-drilling (LWD) sector-based nuclear (density, neutron porosity, photoelectric factor, gamma ray) and array propagation resistivity measurements acquired in $\mathrm{HA} / \mathrm{HZ}$ wells. A challenging synthetic benchmark example confirms improved formation evaluation with the layerbased inversion workflow across hydrocarbon-bearing zones in $\mathrm{HA} / \mathrm{HZ}$ wells, where estimated hydrocarbon pore volume and porosity increase by $10 \%$ and $15 \%$, respectively, with respect to conventional interpretation methods. Furthermore, application of the inversion-based method to a field example of $\mathrm{HZ}$ well across calcite-cemented siltstone layers confirms its advantage over conventional interpretation techniques.

\subsection{INTRODUCTION}

High-angle and horizontal ( $\mathrm{HA} / \mathrm{HZ})$ wells increase exposure of hydrocarbonbearing zones to the wellbore, thereby improving logging data quality and hydrocarbon flow area. Occasionally, imperfect $\mathrm{HZ}$ well placement gives rise to well trajectory

undulations that traverse multiple bed boundaries, whereby borehole measurements 
respond to multiple layer properties at the same time. A detailed understanding of the three-dimensional (3D) relationship between well-log measurement physics and bed geometrical effects is essential to develop new and improved formation evaluation methods in HA/HZ wells (Passey et al., 2005).

Logging-while-drilling (LWD) measurements usually consist of azimuthal image logs in multi-sector tool rotations. With adequate understanding of 3D tool responses and effective volume of investigation (EVOI), a wealth of information, including bed dip, azimuth, and true stratigraphic thickness (TST), can be interpreted from LWD borehole images. The EVOI of nuclear tools, parameterized as effective radial penetration length, $E P L$ (often referred to as depth of image or depth of investigation), axial or vertical resolution, $A R$, and azimuthal aperture, $\Delta \psi$ (Yin et al., 2008), can be estimated from spatial flux sensitivity functions (FSFs, Mendoza et al., 2007; Ijasan et al., 2011). In vertical wells, measurement value is jointly influenced by $E P L$ and $A R$, while bed boundary detection is solely affected by $A R$. Assuming azimuthal homogeneity, $\Delta \psi$ has negligible influence in vertical wells even in the presence of invasion. On the other hand, in $\mathrm{HA} / \mathrm{HZ}$ wells, the three EVOI parameters influence measurement value and boundary detection in different ways depending on the type of measurements (nuclear or resistivity), petrophysical properties, and bed geometrical properties, i.e., dip, stratigraphy, well trajectory, and tool orientation.

Inverse theory has proved valuable for interpretation of logs acquired in vertical wells, particularly across complex lithologies and thinly bedded formations. SanchezRamirez et al. (2010), and Heidari et al. (2012) implemented joint inversion of nuclear and resistivity wireline measurements for reduction of shoulder-bed effects and improved quantitative estimation of petrophysical properties. Mendoza et al. (2012), and Shetty et al. (2012) documented the use of inversion techniques that explicitly consider spatial 
density responses for estimating formation bulk density, $\rho_{b}$, from sector-based LWD dual-detector and compensated density images. Additionally, Mendoza et al. (2010c), and Zhou et al. (2012) showed that measurement integration is necessary for accurate quantitative petrophysical and geometrical interpretation in $\mathrm{HA} / \mathrm{HZ}$ wells.

In this chapter, I implement a nonlinear inversion workflow for combined, quantitative petrophysical and compositional interpretation of LWD sector-based nuclear (density, neutron porosity, photoelectric factor, natural gamma ray) and array propagation resistivity measurements in $\mathrm{HA} / \mathrm{HZ}$ wells. I incorporate nuclear and resistivity models that reproduce 3D measurement responses in $\mathrm{HA} / \mathrm{HZ}$ wells for explicit consideration of relationships characterizing measurement EVOI, bed geometrical effects, and well trajectory. Nuclear measurements are modeled with 3D Monte Carlo (MC)-derived FSFs (Mendoza et al., 2010a; Ijasan et al., 2013), while propagation resistivity responses are numerically simulated using the ARC (Schlumberger Array Resistivity Compensation) forward model. By considering 3D tool responses in the inversion, I effectively reduce bed geometry and well trajectory effects on LWD measurements to obtain layer-by-layer petrophysical characteristics, while taking advantage of image data resolution in $\mathrm{HA} / \mathrm{HZ}$ wells.

Different physical principles governing the various borehole measurements enforce separate tool responses in the subsurface (Ellis and Singer, 2007). For example, nuclear measurements respond to bulk petrophysical properties while the electrical resistivity response is directional in nature. This behavior calls for inversion for separate petrophysical properties within each measurement domain. In other words, I estimate layer-by-layer bulk density, $\rho_{b}$, neutron migration length, $L_{m}$, photoelectric factor (PEF), volumetric concentration of shale, $C_{s h}$, and true electrical conductivity, $\sigma_{t}$, from inversion of sector-based LWD density, neutron porosity, PEF, natural gamma ray (GR), and array 
propagation resistivity measurements, respectively. Subsequently, compositional or multi-mineral analysis is carried out on inverted layer-by-layer petrophysical properties for estimation of grain density, $\rho_{g}$, total porosity, $\phi_{T}$, water saturation, $S_{w}$, and hydrocarbon pore volume, $H P V$, assuming prior knowledge of GR- $C_{s h}$ relationship and Archie's parameters. I document and verify the application of the inversion workflow with (i) a synthetic example in an $82^{\circ}$ up-dip well across known formation composition, (ii) a field example in a HA interval, and (iii) a field example in a $\mathrm{HZ}$ interval.

\subsection{INVERSION-BASED INTERPRETATION WORKFLOW}

The interpretation workflow consists of three main steps. Firstly, I assume a formation geometrical model that is locally described by two-dimensional (2D) structural properties obtained from consolidated geometrical interpretation of sector-based LWD gamma (density, PEF, GR) borehole images. Structural properties consist of bed boundaries, dips, and azimuths for describing the 2D layer-based formation model. In this model, I assume that LWD logs are acquired after minimal formation exposure to borehole environmental conditions, such that mud-filtrate invasion is negligible and formation petrophysical properties are approximately constant and isotropic within each layer structure. Next, I impose the formation geometrical model on nuclear and resistivity measurement domains for separate petrophysical inversion. Finally, a multi-mineral compositional solver is used to estimate layer-by-layer solid and fluid volumetric concentrations from inverted layer properties. Figure 4.1 describes the three steps of the inversion-based interpretation workflow.

\subsubsection{Consolidated Gamma Geometrical Model}

Layer geometrical properties, bed thickness, apparent dip, and apparent azimuth, are calculated from nuclear gamma images, i.e., sector-based density, PEF, and GR (see 
Step I in Figure 4.1). Initially, bed boundaries are detected along well depth and around borehole azimuth from short-spaced, $\rho_{S S}$, long-spaced, $\rho_{L S}$, or compensated, $\rho_{C O}$, density images using a threshold variance algorithm (Uzoh et al., 2009). Selected bed boundaries, resulting from bed boundary planes intersecting a circular borehole, define the "gamma borehole" sinusoids (Yin et al., 2008). Next, a least-squares minimization method (Plumb and Luthi, 1989) is used to estimate bed boundary location, apparent dip, and apparent azimuth from the quadratic cost function written as

$$
\mathbf{C}\left(B_{b}, \theta_{b}, \beta_{b}\right)=\| \begin{aligned}
& \| B_{b}+E P L \tan \theta_{b}-\mathbf{A}_{b, j}+ \\
& \quad\left\{\frac{D}{2}+E P L\right\}\left\{\cos \left(\boldsymbol{\alpha}_{j}-\beta_{b}\right)-\cos \beta_{b}\right\} \tan \theta_{b} \|_{2}^{2},
\end{aligned}
$$

where $j$ and $b$ designate $j$-th sector-based bin and $b$-th bed boundary, respectively, $D$ is borehole diameter, $\boldsymbol{\alpha}_{j}$ is vector of azimuthal bins in degrees, $B_{b}$ is depth-coherent physical bed boundary location at $\alpha_{j}=0^{\circ}$ (north) in measured depth, $\theta_{b}$ is apparent bed dip, and $\beta_{b}$ is apparent bed strike or azimuth; $\mathbf{A}_{b, j}$ is "gamma borehole" sinusoid locations detected using the threshold variance algorithm along borehole depth and around borehole azimuth. Note that $\mathbf{A}_{b, j}$ includes the inherent EPL effect of sector-based measurements, such that equation 4.1 corrects for depth coherence and is easily differentiable to obtain analytical Jacobian gradients in conjunction with nonlinear leastsquares minimization.

Additional sinusoids are detected from PEF and GR images. For single-curve PEF and GR logs, I assume that the detected boundaries are located at the intersection between the bed boundary plane and the hole axis, i.e., $\cos \left(\alpha_{j}-\beta_{b}\right)=0$. This is because single curves cannot distinguish between up-dip or down-dip bed boundaries. The 
complete set of $B_{b}, \theta_{b}$, and $\beta_{b}$, defining the layer-based geometry, is referred to as the consolidated gamma geometrical model. Additionally, particularly in HZ wells with undulating well trajectories, I refine the set of $B_{b}, \theta_{b}$, and $\beta_{b}$ by manually picking more sinusoids from sector-based measurements. Together with well trajectory data or borehole true vertical depth (TVD), I infer the 2D layer stratigraphic properties from the consolidated geometrical model (Griffiths, 2009).

\subsubsection{Measurement Domains: Property Parameterization and Data Space}

Measurement physics of well logs plays an important role in the development of interpretation techniques. Integrated interpretation demands explicit multi-physics characterization because different well logs measure or interact with different formation petrophysical properties and volumes. Neutron porosity, $\phi_{N}$, is primarily governed by hydrogen index (HI) and $L_{m}, \rho_{b}$ by electron density, $\rho_{e}$, PEF by average atomic number, GR by naturally occurring radioactivity of thorium, uranium, and potassium; and apparent resistivity, $R_{a p p}$, by $\sigma_{t}$ of the formation (Ellis and Singer, 2007). Consequently, in each measurement domain, I estimate layer-by-layer physical properties governing each measurement. The parameterization involves $\rho_{b}, L_{m}, \mathrm{PEF}, C_{s h}$, and $\sigma_{t}$ for characterization of density $\left(\rho_{S S}, \rho_{L S}, \rho_{C O}\right)$, neutron detector count rate and porosity (ncps, $\left.f c p s, \phi_{N}\right), \mathrm{PEF}$, natural GR, and apparent electrical conductivity $\left(1 / R_{\text {app }}\right)$ measurements, respectively. In this parameterization, the neutron porosity tool is calibrated with Schlumberger's Nuclear Parameter calculator (SNUPAR, McKeon et al., 1989), i.e., for a given neutron source, $L_{m}$ is calculated in base-cases of water-filled limestone, sandstone, and dolomite porosity units. For the examples discussed in this chapter, a linear approximation scale is assumed for conversion of API GR measurements to $C_{s h}$. 
Depending on the available measurements, nuclear data are assimilated into the interpretation in the following manner:

(1) sector binning scheme, e.g. single curves or sector-based (quadrant or multiple bins), and

(2) detector data type, e.g. neutron, density dual-detector (ncps-fcps, $\left.\rho_{S S^{-}} \rho_{L S}\right)$; compensated $\left(\rho_{C O}, \phi_{N}\right)$; or single-detector lithology measurements (PEF, GR).

In resistivity data space, all array propagation channels of phase-shift, attenuation, 400 $\mathrm{kHz}$ and $2 \mathrm{MHz}$ are used for $\sigma_{t}$ inversion, i.e., a total of 20 channels. Appendix B provides further details about the data space.

\subsubsection{Overview of Inversion Method}

In the second step of my workflow (Step II in Figure 4.1), I impose the formation geometrical model on nuclear and resistivity measurements for separate nonlinear inversion within each measurement domain. Accordingly, the quadratic misfit cost function to be minimized is

$$
\mathbf{C}(\mathbf{p})=\|\mathbf{e}(\mathbf{p})\|_{2}^{2}+\lambda^{2}\left\|\mathbf{p}-\mathbf{p}^{0}\right\|_{2}^{2}
$$

where $\mathbf{p}=\mathbf{p}\left(\rho_{b}, L_{m}, \mathrm{PEF}, C_{s h}, \sigma_{t}\right)$ is the vector of layer physical properties, $\mathbf{p}^{0}$ is the initial guess, $\mathbf{e}(\mathbf{p})=\mathbf{d}(\mathbf{p})-\mathbf{d}_{s}$ is the vector of data misfit whereby $\mathbf{d}(\mathbf{p})$ is numerically simulated data while $\mathbf{d}_{s}$ designates the available measurements, and $\lambda$ is a regularization (stabilization) parameter calculated with the generalized cross-validation (GCV) method (Hansen, 1998) and intended to provide selective weighting to the two additive terms included in the quadratic cost function. The ensuing expression for iterative nonlinear 
minimization of equation 4.2, using the regularized Levenberg-Marquardt method (Aster et al., 2005) becomes

$$
\left[\mathbf{J}^{T} \mathbf{J}+\lambda^{2} \mathbf{W}_{\mathrm{m}}^{T} \mathbf{W}_{\mathrm{m}}\right] \Delta \mathbf{p}=-\mathbf{J}^{T} \mathbf{e}+\lambda^{2} \mathbf{W}_{\mathrm{m}}^{T} \mathbf{W}_{\mathrm{m}} \mathbf{p}^{k},
$$

where $\Delta \mathbf{p}=\mathbf{p}^{k+1}-\mathbf{p}^{k}$ and $k$ designates the $k$-th iteration, $\mathbf{J}$ is the Jacobian (sensitivity) matrix, and $\mathbf{W}_{\mathrm{m}}$ is the model weighting matrix used to control the importance of each layer where entries of its diagonal are inversely proportional to relative layer thicknesses such that emphasis can be placed on thin beds to improve uniqueness across thinlylaminated intervals; when $\mathbf{W}_{\mathrm{m}}$ is the identity matrix all layers have equal importance. Convergence is achieved when $\frac{d\left(\log \|\mathbf{e}(\mathbf{p})\|_{2}^{2}\right)}{d k}=0$.

In the nuclear measurement domain, I construct $\mathbf{J}$ from 3D FSFs (Mendoza et al., 2012). It follows for density, PEF, and GR measurements that their FSFs are approximately constant for varying formation $\rho_{b}, \mathrm{PEF}$, and $C_{s h}$, respectively (Mendoza et al., 2010a; Ijasan et al., 2011). This approximation is valid across bed boundaries in HA/HZ wells. Consequently, J for density, PEF, and GR measurements are assumed constant, whereby the separate inversion of density, PEF, and GR logs in equation 4.3 simplify to linear minimization of the quadratic cost function in equation 4.2 (Mendoza et al., 2012; Heidari et al., 2012). On the other hand, as discussed in Chapter 3, neutron porosity FSFs exhibit significant flux perturbations at bed boundaries, across varying formation HI, and in the presence of borehole environmental effects. I account for these flux perturbations by augmenting neutron FSFs with the recently developed semianalytical fast diffusion flux-difference (DFD) approximation in Chapter 3 (Ijasan et al., 2013), so that $\mathbf{J}$ for inverting neutron porosity consists of transport and diffusion parts. 
In the resistivity measurement domain, $\mathbf{J}$ is numerically calculated from finite-difference approximations of partial derivatives. Appendix B provides further descriptions about the entries of $\mathbf{J}$ for inversion of nuclear and resistivity measurements.

\subsubsection{Compositional Petrophysics}

The final step in my interpretation workflow (Step III in Figure 4.1) involves estimation of layer-by-layer $\rho_{g}, \phi_{T}$, and $H P V$. I invoke a SNUPAR-based compositional solver (Heidari et al., 2012) coupled with appropriate $S_{w}$ models (e.g., Archie or shalysand models with known Archie's parameters) and GR- $C_{s h}$ relationships (e.g., linear; Larionov, 1969; or Clavier et al., 1971, etc.) in the estimation of solid and fluid compositions from inverted layer-by-layer properties, $\mathbf{p}\left(\rho_{b}, L_{m}, \mathrm{PEF}, C_{s h}, \sigma_{t}\right)$. The compositional solver takes advantage of the deterministic relationship between complex rock/fluid mixtures and nuclear physical properties, quantified by the SNUPAR program. Appendix B describes the formulations of the SNUPAR-based solver. Note that other commercial multi-mineral solvers could be applied at this step of the interpretation workflow.

\subsection{Application to High-Angle and Horizontal Wells}

This section describes the application of the inversion-based interpretation workflow. Using a synthetic example in $82^{\circ}$ up-dip well, I compare inversion-based interpretation to conventional interpretation. The analysis also considers the effects of bed-geometry uncertainty on inverted layer-by-layer physical properties. Furthermore, I apply the workflow to LWD nuclear and resistivity measurements acquired in highlydeviated and horizontal well intervals of a hydrocarbon field located in West Africa. 


\subsubsection{Synthetic Benchmark Example}

I assume a layered synthetic model, of depth-periodic compositions and varying TST, to describe shoulder-bed, EVOI, and bed dip effects on nuclear and resistivity measurements acquired in $\mathrm{HA} / \mathrm{HZ}$ wells. For simplicity but without sacrifice of generality, I invoke Archie's saturation model. Tables 4.1 and 4.2 describe layer compositions, petrophysical properties, and Archie's parameters assumed in the synthetic example. With an up-dip well of $82^{\circ}$ inclination and bed azimuths of $30^{\circ}$, borehole nuclear logs are numerically simulated using the FSF technique and generic LWD logging tools (Mendoza et al., 2007, Ijasan et al., 2011; 2013), while array propagation resistivity curves are simulated using the ARC forward model. In the data space, I assume 16-sector bins for sector-based density $\left(\rho_{S S}, \rho_{L S}, \rho_{C O}\right)$ and GR measurements, quadrantsector bins for neutron porosity and detector count rate $\left(\phi_{N}, n c p s, f c p s\right)$ measurements, and single curves for PEF and array propagation resistivity channels. Measurements are sampled every $0.25 \mathrm{ft}$ along the well trajectory. Additionally, $10 \%$ random noise is added to the synthetic measurements to mimic actual field logs. Figure 4.2 shows the numerically simulated synthetic data.

As discussed in the workflow, the formation geometrical model is obtained from bed boundary detection of $\rho_{S S}$ image, GR image, and PEF log. The geometrical model is then imposed on nuclear and resistivity measurement domains for layer-based separate inversion. Figure 4.3 shows layer-based physical properties $\left(\rho_{b}, L_{m}, C_{s h}\right.$ PEF, $\left.\sigma_{t}\right)$ from inversion, together with solid and fluid concentrations from the compositional solver. Additionally, green error bars in panels a-e identify $95 \%$ inversion confidence intervals, estimated using the model covariance in equation B.1 of Appendix B. Error bars, quantifying non-uniqueness and stability of the inversion, are largest across the 2.08-in. (5.28-cm) beds of layers VI and VII (Table 4.1), especially for inverted $L_{m}$ (panel b) and 
$\sigma_{t}$ (panel e). This behavior is due to the fact that the consolidated geometrical model is below EVOI resolution of the neutron and resistivity logs. Such an EVOI effect manifests itself as non-uniqueness in inverted layer properties. Note that true model properties, identified with dashed red lines, are within confidence intervals. Panel $\mathrm{f}$ of Figure $\mathbf{4 . 3}$ shows layer-by-layer volumetric concentrations of solid and fluid compositions, where black error bars identify confidence intervals, per component per layer, propagated from inversion and SNUPAR-based solver. Volumetric concentrations in panel $\mathrm{f}$ are in agreement with synthetic properties in Tables 4.1 and 4.2. To appraise data misfit, I compare numerically simulated LWD measurements across the solid and fluid compositions in panel $f$ of Figure 4.3 to the original synthetic data in Figure 4.2. Figure 4.4 shows these comparisons where average misfits and misfit standard deviations are below $1.5 \%$ and $5 \%$, respectively, for nuclear measurements and $1 \%$ and $6 \%$, respectively, for apparent resistivity measurements.

Next, I perform the conventional interpretation approach applied in vertical wells on synthetic logs. Conventional interpretation involves multi-mineral analysis of depthmatched nuclear logs and 40-in. high-frequency phase-shift array resistivity $(\mathrm{P} 40 \mathrm{H}) \log$, using the SNUPAR-based solver and Archie's saturation model. Figure 4.5 shows comparisons of $\phi_{T}, S_{w}, \rho_{g}$, and $H P V$ obtained from conventional and inversion-based interpretation. In panel f, green and black shaded zones identify where conventional interpretation under- and over-estimates $H P V$, respectively. I observe $H P V$ differences, as much as $15 \%$ pore volume (originating from inaccurate interpretation of $\phi_{T}$ and $S_{w}$ by 15 $\%$ and $10 \%$, respectively), are particularly accentuated at bed boundaries and across thin layers V to VIII.

Consolidated geometrical interpretation of $B_{b}, \theta_{b}$, and $\beta_{b}$, described earlier, is a crucial step for the inversion-based method. By introducing 10 realizations of random 102 
perturbations in $B_{b}, \theta_{b}$, and $\beta_{b}$, I investigate effects of bed-geometry uncertainty on inverted $\rho_{b}$ and $L_{m}$ from dual-detector and compensated density and neutron porosity measurements. Figure 4.6 describes the perturbed geometrical model, and subsequently inverted $\rho_{b}$ and $L_{m}$. I observe, in panels $\mathrm{d}-\mathrm{f}$, that thin layers are very sensitive to perturbations in the geometrical model. These instability effects on inverted $\rho_{b}$ and $L_{m}$ are as high as $0.4 \mathrm{~g} / \mathrm{cm}^{3}$ and $7 \mathrm{~cm}$, respectively, corresponding to $23 \mathrm{pu}$ and $15 \mathrm{pu}$ in limestone units, for an average perturbation of \pm 3.5 in. in $B_{b}, \pm 2.5^{\circ}$ in $\theta_{b}$, and $\pm 1.5^{\circ}$ in $\beta_{b}$, in an $82^{\circ}$ well. Such a behavior is qualitatively consistent with error bars in Figure 4.3, and is important learning for layer-based inversion in $\mathrm{HA} / \mathrm{HZ}$ wells, thus emphasizing the need for accurate geometrical interpretation (Yin et al., 2006; Griffiths, 2009).

\subsubsection{Field Case Example in Highly-Deviated Well}

The case of study is a hydrocarbon field located in West Africa, consisting of alternating laminations of argillaceous limestone and calcite-cemented feldspathic siltstones of 1 to 2-ft TST (0.3 to 0.6-m; Mendoza et al., 2012). Figure 4.7 summarizes the LWD nuclear and resistivity measurements acquired in a HA interval of the field example. The HA interval is below the free oil-water contact in the hydrocarbon column, thus primarily saturated with water such that bottom sector density $\left(\phi_{D}\right)$ and neutron $\left(\phi_{N}\right)$ porosity logs almost overlap across the interval (panel d). Table 4.3 describes multimineral and fluid component models assumed in the West Africa hydrocarbon field. Borehole density images are acquired on a 16-sector binning scheme, where the well trajectory varies between $78^{\circ}$ and $82^{\circ}$ for the interval. I observe that $\rho_{S S}$ and $\rho_{L S}$ images, in panels a and b of Figure 4.7, respectively, are marred by standoff at the up (U) sector bins. Shetty et al. (2012) described model-based inversion of $\rho_{S S}$ and $\rho_{L S}$ images for interpretation of formation density and borehole shape, where borehole standoff is an 
inverted property. In this chapter, I focus on petrophysical and compositional interpretation whereby the $\rho_{C O}$ image, in panel c of Figure 4.7, suffices for my inversion workflow, given that it utilizes $\rho_{S S}$ and $\rho_{L S}$ for compensation and correction for borehole standoff.

The gamma bed boundary sinusoids for the consolidated geometrical model are superposed on the $\rho_{C O}$ image. Bed boundaries sinusoids obtained from $\rho_{C O}$ image only are identified with white lines, while those obtained from $\rho_{C O}$ image or/and PEF and GR logs are identified with magenta and green lines, respectively. It is worth noting that the consolidated geometrical model characterizes local petrophysical boundaries that represent layers of distinct petrophysical properties and not just distinct bulk densities. For example, within XX15 - XX25 ft and XY33 - XY50 ft, the $\rho_{C O}$ image shows a relatively constant response, while GR and PEF logs indicate variations in lithology.

In addition to $\rho_{C O}, \mathrm{PEF}$, and GR measurements, single curve $\phi_{N}$ and 20 channels of array propagation resistivity curves are available for inversion. It follows from Appendix $\mathbf{B}$ that $\mathbf{J}_{s d}$ is implemented for estimating $C_{s h}$ and PEF, while $\mathbf{J}_{C O}$ is implemented for inverting $\rho_{b}$ and $L_{m}$. In Figure 4.8, I show inverted layer-by-layer properties, $\mathbf{p}\left(\rho_{b}, L_{m}, C_{s h}, \mathrm{PEF}, \sigma_{t}\right)$, and their confidence intervals. Accordingly, the dashed red lines represent predicted SNUPAR properties upon convergence of the multimineral analysis. An interesting observation is that error bars on estimated $L_{m}, \mathrm{PEF}$, and $C_{s h}$ are relatively larger than error bars on estimated $\rho_{b}$ and $\sigma_{t}$. This behavior is primarily due to data resolution per measured depth; given that $\phi_{N}, \mathrm{PEF}$, and GR are single curves, while $\rho_{C O}$ and $R_{a p p}$ consist of 16 -sector binning curves and 20 channels, respectively. As discussed, the purpose of inversion is to correct the LWD measurement for well trajectory, shoulder-bed, and EVOI effects, such that the inverted parameters represent true bed petrophysical properties. True bed properties are then analyzed with the 104 
SNUPAR-based multi-mineral solver, assuming Poupon-Leveaux saturation model (Poupon and Leveaux, 1971; see Table 4.3), to obtain solid and fluid volumetric concentrations. Panel a of Figure 4.9 describes estimated solid and fluid volumetric concentrations, while panels b, c, and d show estimated $\phi_{T}, S_{w}$, and $\rho_{g}$, respectively.

\subsubsection{Field Case Example in Horizontal Well}

Reliable interpretation of logs acquired in $\mathrm{HZ}$ wells has been challenging due to limited understanding of $\mathrm{HZ}$ well trajectory effects on log responses. Figure 4.10 shows well data and trajectory in a HZ interval of the field example. The $\rho_{C O}$ image, in panel a of Figure 4.10, exhibits the classic "bull's-eye" feature between ZY35 and ZA60 ft, particularly characteristic of undulating HZ wells. Such a feature is produced as the borehole traverses in and out of the clean and silty limestone layers, in up- and down-dip drilling directions, respectively. Panel a also shows the gamma borehole bed boundaries, where dashed white lines represent apparent borehole sinusoids for inferring stratigraphic properties, while solid white lines represent intersection of the well trajectory with stratigraphic boundaries. Panel $\mathrm{f}$ of Figure 4.10 describes the well trajectory and 2D curtain section of the layer-based formation model obtained from structural interpretation of the $\rho_{C O}$ "bull's-eye" feature. The formation model is then imposed on nuclear and resistivity domains for layer-based inversion in TVD.

Figure 4.11 shows the inverted properties across the HZ interval in layer-by-layer TVD. The petrophysical layers from Z03 - Z07 ft in TVD are sampled multiple times by the HZ section such that $95 \%$ confidence intervals (green error bars in Figure 4.11) within these layers are relatively smaller than for other layers. This is one advantage of $\mathrm{HZ}$ well acquisition, i.e., to improve logging data quality within zones of interest. 
Nuclear and $\mathrm{P} 40 \mathrm{H}$ resistivity logs, shown in Figure 4.10, are re-sampled in TVD and input directly to the SNUPAR-based solver for conventional analysis. Figure 4.12 compares estimated petrophysical properties obtained from conventional interpretation to inversion-based interpretation. Similar to observations in the synthetic example, conventional analysis over-estimates and under-estimates $H P V$ (panel f) in water and hydrocarbon saturated layers, respectively. Below Z03 ft, $S_{w}$ (panel d) estimated from conventional analysis is significantly influenced by shoulder-bed effects, so much that $H P V$ is over-estimated by $10 \%$. This behavior is attributed to large EVOI of resistivity measurements. Note that the HZ interval spans a TST interval of about $13 \mathrm{ft}(4 \mathrm{~m})$, in comparison to approximately $6.5-\mathrm{ft}(2.0-\mathrm{m})$ EPL of the resistivity response. This implies, as a consequence of the EVOI effect, that the resistivity measurement samples multiple layers simultaneously. Additionally, in the $\mathrm{HZ}$ section, layers are completely parallel to the borehole such that anisotropy influences the apparent resistivity measurements. As described in Appendix $\mathbf{B}, \mathbf{J}_{\sigma}$ is numerically derived for the well trajectory in panel $\mathrm{f}$ of Figure 4.10 such that anisotropy and polarization horns are inherently considered in the finite-difference approximations.

To verify my inversion results, I perform numerical simulations of LWD measurements across the layer-by-layer properties in panel a of Figure 4.12 using the well trajectory in panel f of Figure 4.10. Figure 4.13 compares field and the numerically simulated LWD measurements. I observe good agreement between field and numerically simulated measurements, with average data misfits and misfit standard deviations below $0.5 \%$ and $5 \%$, respectively, for nuclear measurements, and $3 \%$ and $10 \%$, respectively, for resistivity measurements. 


\subsection{SUMMARY AND DISCUSSION}

The inversion-based interpretation workflow consists of three steps: (I) consolidated geometrical interpretation of LWD nuclear gamma (density, PEF, GR) measurements to construct a 2D layer-based formation model; (II) separate nonlinear inversion of LWD nuclear and resistivity measurements, within each measurement domain; and (III) layer-by-layer compositional interpretation of estimated properties.

The consolidated geometry describes bed boundaries that define layers of locally distinct petrophysical properties. This model is constructed by calculating the local variance of density, PEF, and natural GR logs. As shown in equation 4.1, $B_{b}$ is depthcorrected for EPL such that it is independent of the respective gamma measurement used for its detection. Density, PEF, and natural GR measurements are good candidates for geometrical interpretation because of their high $A R$, shallow EPL, and focused $\Delta \psi$. Furthermore, high resolution borehole images, e.g., FMI (formation micro-imager) measurements, and resistivity polarization horns can be used to enhance the geometrical model. The accuracy in structural and stratigraphic interpretation of sector-based LWD measurements is very crucial. Yin et al. (2006) described the sensitivity of absolute error in apparent dip to TST estimation, where a $1^{\circ}$ error in dip can give rise to TST relative errors of $20 \%$ and $100 \%$ in HA and HZ wells, respectively. With the synthetic example, I showed that uncertainty in bed-geometry, especially across thinly bedded formations, could give rise to errors as high as $23 \mathrm{pu}$ and $15 \mathrm{pu}$ in the inversion of density and neutron porosity measurements, respectively.

Borehole measurements acquired in $\mathrm{HA} / \mathrm{HZ}$ wells are significantly influenced by well deviation effects, such that estimated $H P V$ from traditional conventional analysis can give rise to errors as high as $15 \%$ and $10 \%$ in $\mathrm{HA}$ and $\mathrm{HZ}$ intervals, respectively. I verified that inversion corrects LWD measurements for well trajectory effects and 
improves formation evaluation in $\mathrm{HA} / \mathrm{HZ}$ wells. The inversion-based workflow is fast and easy to implement with the measurement domain and property parameterization platform. It promotes efficient assimilation of other borehole measurements, such as acoustic, magnetic resonance, and geochemical logs. Additionally, the platform enforces stability of inversion calculations and explicit multi-physics characterization, within each domain, for subsequent estimation of solid and fluid compositions. Furthermore, in the resistivity domain, I observed that inversion of apparent conductivity measurements $\left(1 / R_{\text {app }}\right)$ is most stable in comparison to inversion of apparent resistivity $\left(R_{\text {app }}\right)$ and its logarithm $\left(\log R_{a p p}\right)$. In the gradient-based search for a local minimum, $k$-th iterations with non-positive layer-by-layer $\sigma_{t}$ solutions are reset, i.e., $\sigma_{t}^{k}=\sigma_{t}^{k-1} \forall \sigma_{t}^{k} \leq 0$. For the assessment of formation anisotropy, elements of $\mathbf{J}_{\sigma}$ are obtained from perturbations in apparent resistivity due to perturbations in vertical and horizontal conductivities, $\mathbf{p}\left(\sigma_{v}, \sigma_{h}\right)$. Appendix $\mathbf{C}$ describes the appraisal of formation anisotropy and calculation of vertical and horizontal resistivities from layer-based inversion of propagation resistivity measurements.

The physics-based SNUPAR multi-mineral solver, in comparison to probabilitybased commercial mineral-solvers, calculates nuclear measurement parameters using semi-analytical multi-group relationships that describe nuclear transport phenomena. To improve convergence of the solver, properties of solid and fluid components can be enhanced using information from core and XRD (x-ray diffraction) measurements.

The objective of $\mathrm{HA} / \mathrm{HZ}$ wells is to improve logging data resolution and increase exposure of hydrocarbon-bearing zones to the wellbore. This important advantage is invoked by my inversion workflow. I observe from Figures 4.8, 4.9, 4.11, and 4.12 that data resolution in terms of $\mathrm{HZ}$ well sampling, borehole imaging, and multi-array channels can significantly improve confidence in estimated layer-by-layer petrophysical 
properties. Additionally, the inversion-based workflow is implemented for locallydescribed 2D geometrical curtain sections. Interpreting extended logging depth intervals will require multiple runs of the inversion-based workflow with several localized curtain sections.

\subsection{CONCLuSIONS}

I introduced an efficient inversion-based workflow for combined, quantitative interpretation of LWD nuclear and resistivity measurements acquired in $\mathrm{HA} / \mathrm{HZ}$ wells. The workflow estimates layer-by-layer petrophysical properties $\left(\rho_{b}, L_{m}, \mathrm{PEF}, C_{s h}, \sigma_{t}\right)$, using separate nonlinear inversion of nuclear (dual-detector, compensated, singledetector; density, neutron porosity, PEF, GR) and resistivity (array propagation) measurements. Using a SNUPAR-based multi-mineral solver, together with appropriate $S_{w}$ model and GR- $C_{s h}$ relationship, the inverted layer-by-layer petrophysical properties were subsequently used to estimate solid composition and hydrocarbon saturation.

Even though well deviation increases exposure of formation to wellbore, interpretation in $\mathrm{HA} / \mathrm{HZ}$ wells is significantly hindered by well trajectory, 3D bed geometry, shoulder-bed, and EVOI effects. The main purpose of inversion is to correct and minimize these geometrical effects in LWD measurements, so that layer-by-layer properties are due to petrophysical effects only, while taking advantage of high data resolution. The examples considered in this chapter indicated that conventional analysis (developed for vertical wells) often yields inaccurate estimation of $H P V$ in $\mathrm{HA} / \mathrm{HZ}$ wells. 
Table 4.1: Depth-periodic layer properties, compositions, and thicknesses assumed in the synthetic example.

\begin{tabular}{|c|c|c|c|c|c|c|c|}
\hline \multirow{3}{*}{ Layer } & \multirow{3}{*}{$\begin{array}{c}\text { Layer } \\
\text { TST (in.) }\end{array}$} & \multicolumn{6}{|c|}{ Volumetric concentrations (\%) } \\
\hline & & \multicolumn{4}{|c|}{ Solids } & \multicolumn{2}{|c|}{ Fluids } \\
\hline & & Calcite & Dolomite & Kaolinite & Quartz & $\begin{array}{l}\text { Hydro- } \\
\text { carbon }\end{array}$ & Water \\
\hline I & 20.84 & 38 & 40 & 10 & 10 & 0 & 2 \\
\hline II & 5.68 & 5 & 5 & 10 & 50 & 29 & 1 \\
\hline III & 5.68 & 10 & 10 & 30 & 40 & 5 & 5 \\
\hline IV & 5.68 & 38 & 40 & 10 & 10 & 0 & 2 \\
\hline $\mathbf{V}$ & 2.08 & 5 & 5 & 10 & 50 & 29 & 1 \\
\hline VI & 2.08 & 10 & 10 & 30 & 40 & 5 & 5 \\
\hline VII & 2.08 & 38 & 40 & 10 & 10 & 0 & 2 \\
\hline VIII & 2.08 & 5 & 5 & 10 & 50 & 29 & 1 \\
\hline IX & 5.68 & 10 & 10 & 30 & 40 & 5 & 5 \\
\hline $\mathbf{X}$ & 5.68 & 38 & 40 & 10 & 10 & 0 & 2 \\
\hline XI & 8.32 & 5 & 5 & 10 & 50 & 29 & 1 \\
\hline XII & 4.16 & 10 & 10 & 30 & 40 & 5 & 5 \\
\hline XIII & 20.84 & 38 & 40 & 10 & 10 & 0 & 2 \\
\hline \multicolumn{2}{|c|}{$\begin{array}{c}\text { Gamma ray } \\
\text { response (API) }\end{array}$} & 6 & 2 & 600 & 30 & 0 & 0 \\
\hline \multicolumn{2}{|c|}{$\begin{array}{c}\text { Component } \\
\text { density }\left(\mathrm{g} / \mathrm{cm}^{3}\right)\end{array}$} & 2.71 & 2.87 & 2.41 & 2.65 & 0.75 & 1 \\
\hline \multicolumn{2}{|c|}{$\begin{array}{l}\text { Component } \\
\text { formula }\end{array}$} & $\mathrm{CaCO}_{3}$ & $\mathrm{CaMg}\left(\mathrm{CO}_{3}\right)_{2}$ & $\mathrm{Al}_{4} \mathrm{Si}_{4} \mathrm{O}_{10}(\mathrm{OH})_{8}$ & $\mathrm{SiO}_{2}$ & $\mathrm{C}_{8} \mathrm{H}_{18}$ & $\mathrm{H}_{2} \mathrm{O}$ \\
\hline
\end{tabular}


Table 4.2: Petrophysical properties assumed in the synthetic example.

\begin{tabular}{|c|c|c|c|c|c|c|c|c|}
\hline \multicolumn{9}{|c|}{ Petrophysical Properties } \\
\hline Layer & $\begin{array}{c}\text { Bulk } \\
\text { density, } \\
\rho_{b} \\
\left(\mathrm{~g} / \mathrm{cm}^{3}\right)\end{array}$ & $\begin{array}{l}\text { Migration } \\
\text { length, } L_{m} \\
\quad(\mathrm{~cm})\end{array}$ & $\begin{array}{c}\text { Photoelectric } \\
\text { factor, PEF } \\
\text { (b/e) }\end{array}$ & $\begin{array}{c}\text { Gamma } \\
\text { ray } \\
\text { response, } \\
G R \\
(\mathrm{API})\end{array}$ & \multicolumn{2}{|c|}{$\begin{array}{c}\text { True } \\
\text { resistivity, } \\
R_{t}(\Omega-m)\end{array}$} & $\begin{array}{c}\text { Total } \\
\text { porosity, } \\
\phi_{T}(\%)\end{array}$ & $\begin{array}{c}\text { Water } \\
\text { saturation, } \\
S_{w}(\%)\end{array}$ \\
\hline $\begin{array}{l}\text { I, IV, } \\
\text { VII, X, } \\
\text { XIII }\end{array}$ & 2.7038 & 21.63 & 3.58 & 66.08 & \multicolumn{2}{|c|}{3.54} & 2 & 100 \\
\hline $\begin{array}{c}\text { II, V, } \\
\text { VIII, } \\
\text { XI }\end{array}$ & 2.0725 & 14.47 & 1.86 & 75.40 & \multicolumn{2}{|c|}{54.77} & 30 & 3 \\
\hline $\begin{array}{l}\text { III, } \\
\text { VI, IX, } \\
\text { XII }\end{array}$ & 2.4285 & 16.49 & 2.17 & 192.80 & \multicolumn{2}{|c|}{1.27} & 10 & 50 \\
\hline \multicolumn{9}{|c|}{ Archie's parameters } \\
\hline \multicolumn{2}{|c|}{$\begin{array}{l}\text { Archie's Winsauer } \\
\text { constant, } a\end{array}$} & \multicolumn{2}{|c|}{$\begin{array}{l}\text { Archie's porosity } \\
\text { exponent, } m\end{array}$} & \multicolumn{2}{|c|}{$\begin{array}{l}\text { Archie's saturation } \\
\quad \text { exponent, } n\end{array}$} & \multicolumn{3}{|c|}{$\begin{array}{c}\text { Connate water resistivity, } R_{w} \\
(\Omega-\mathrm{m})\end{array}$} \\
\hline & 1 & \multicolumn{2}{|c|}{1.5} & \multicolumn{2}{|l|}{2} & \multicolumn{3}{|c|}{0.01} \\
\hline
\end{tabular}


Table 4.3: Multi-mineral and fluid component models assumed in the West Africa hydrocarbon field examples.

\begin{tabular}{|c|c|c|c|c|c|c|c|c|c|}
\hline & & \multicolumn{8}{|c|}{ Multi-mineral and fluid models for field examples } \\
\hline & & \multicolumn{5}{|c|}{ Solids } & \multicolumn{3}{|c|}{ Fluids } \\
\hline & & \multicolumn{2}{|c|}{ Limestone } & \multicolumn{2}{|c|}{$\begin{array}{c}\text { Orthoclase } \\
\text { feldspar }\end{array}$} & Sandstone & \multicolumn{2}{|c|}{ Hydrocarbon } & Water \\
\hline $\begin{array}{r}\text { Compo } \\
\text { form }\end{array}$ & $\begin{array}{l}\text { ent } \\
\text { a }\end{array}$ & \multicolumn{2}{|c|}{$\mathrm{CaCO}_{3}$} & \multicolumn{2}{|c|}{$\mathrm{KAlSi}_{3} \mathrm{O}_{8}$} & $\mathrm{SiO}_{2}$ & \multicolumn{2}{|l|}{$\mathrm{C}_{8} \mathrm{H}_{18}$} & $\mathrm{H}_{2} \mathrm{O}$ \\
\hline $\begin{array}{l}\text { Compo } \\
\text { density }\end{array}$ & $\begin{array}{l}\text { ent } \\
\left.\mathrm{cm}^{3}\right)\end{array}$ & \multicolumn{2}{|c|}{2.71} & & 2.52 & 2.65 & \multicolumn{2}{|l|}{0.75} & 1 \\
\hline $\begin{array}{r}\text { Gamm: } \\
\text { response }\end{array}$ & $\begin{array}{l}\text { ray } \\
\text { API) }\end{array}$ & \multicolumn{2}{|c|}{10} & & 400 & 50 & \multicolumn{2}{|l|}{0} & 0 \\
\hline \multicolumn{10}{|c|}{ Poupon-Leveaux saturation parameters } \\
\hline $\begin{array}{c}\text { Constant, } \\
a\end{array}$ & \multicolumn{2}{|c|}{$\begin{array}{c}\text { Porosity } \\
\text { exponent, } m\end{array}$} & $\begin{array}{l}\text { Satura } \\
\text { expone }\end{array}$ & & \multicolumn{2}{|c|}{$\begin{array}{c}\text { Connate water } \\
\text { resistivity, } R_{w}(\Omega-\mathrm{m})\end{array}$} & $\begin{array}{c}\text { Shale } \\
\text { resistivity, } \\
R_{s h}(\Omega-\mathrm{m})\end{array}$ & & $\begin{array}{l}\text { Shale } \\
\text { orosity, } \\
p_{s h}(\%)\end{array}$ \\
\hline 1 & \multicolumn{2}{|c|}{2} & \multicolumn{2}{|c|}{2} & \multicolumn{2}{|c|}{0.0747} & 0.3614 & & 8.27 \\
\hline
\end{tabular}




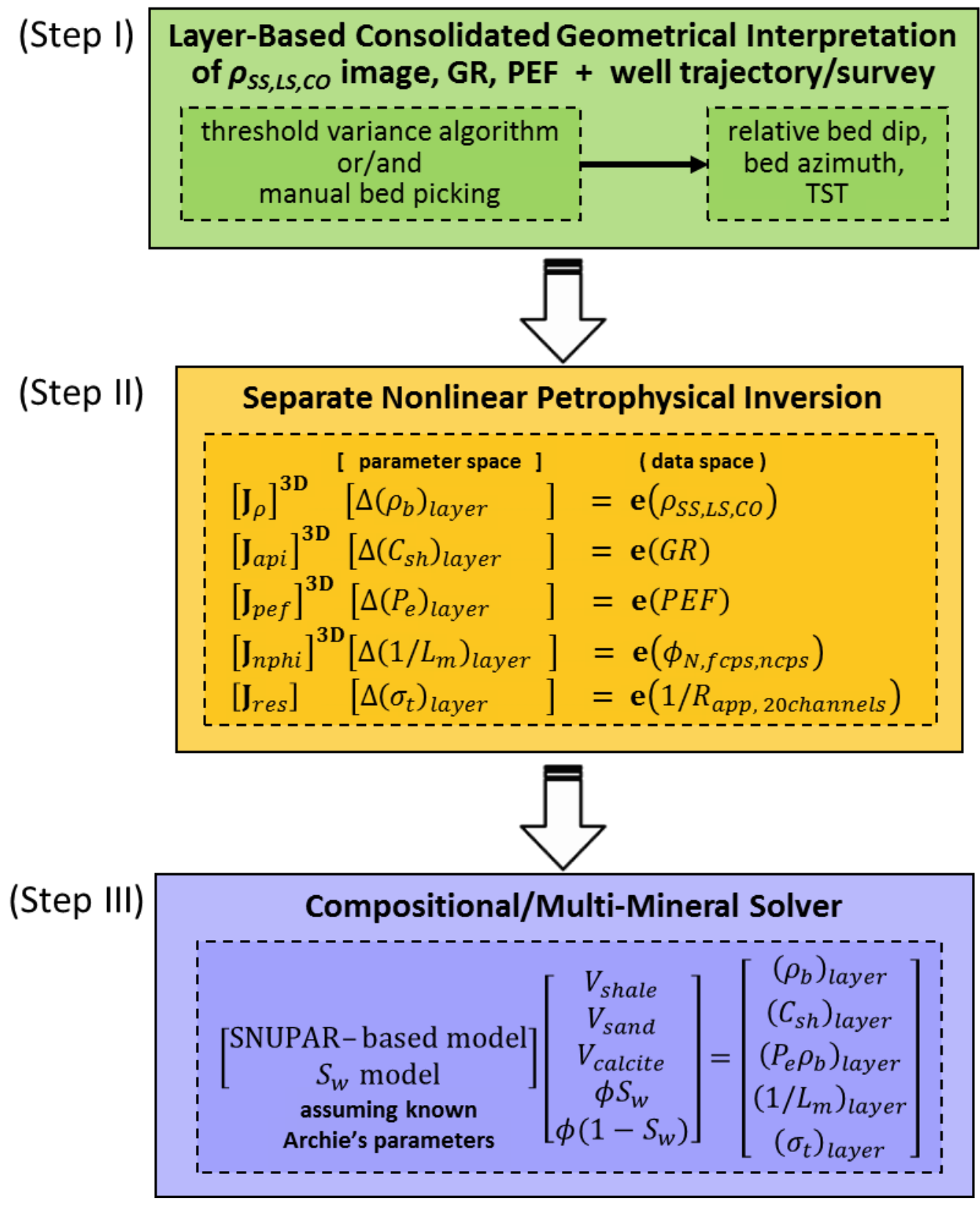

Figure 4.1: Inversion-based workflow for interpretation of LWD nuclear and resistivity measurements acquired in $\mathrm{HA} / \mathrm{HZ}$ wells. 

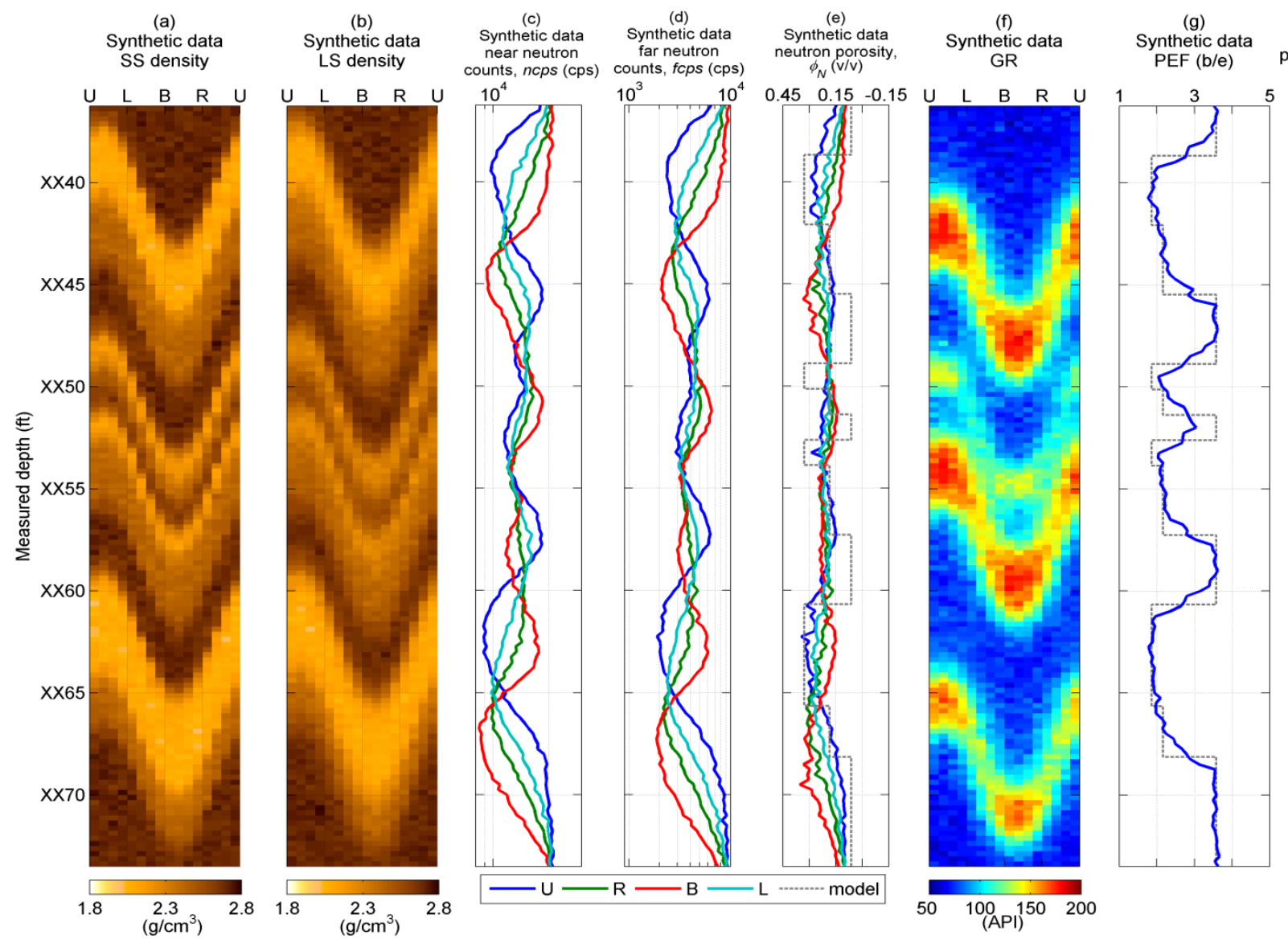

Synthetic $2 \mathrm{MHz}$
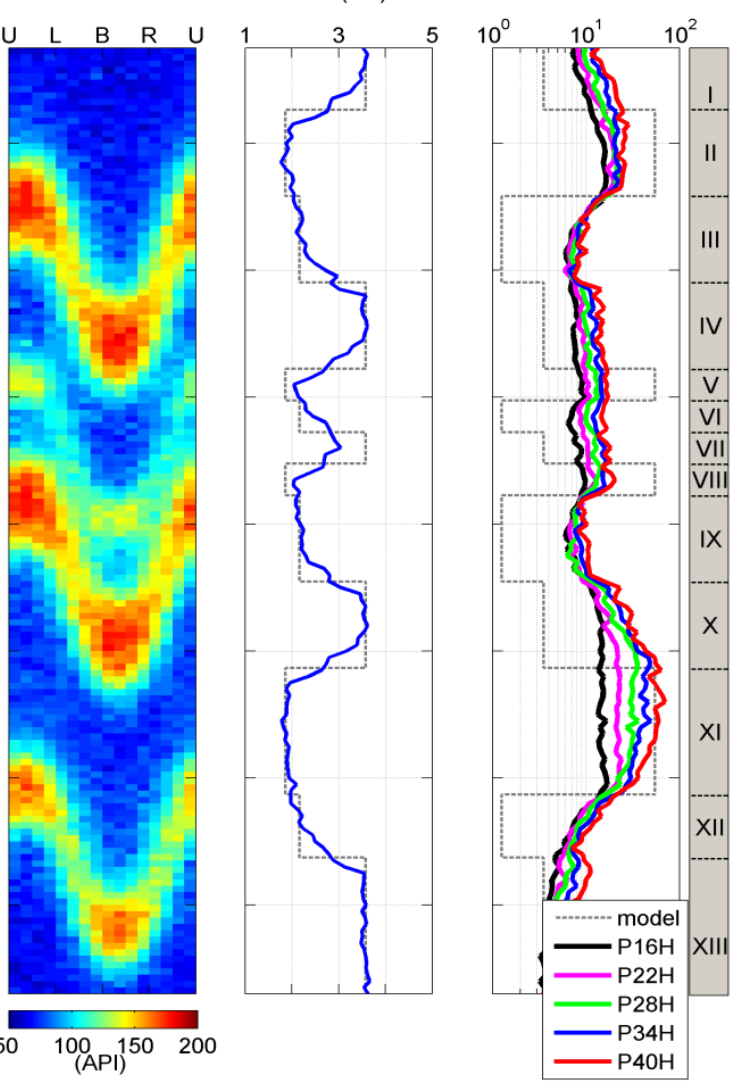

Figure 4.2: $\quad$ Numerically simulated LWD nuclear and resistivity measurements across a synthetic model. (a) Short-spaced density image, $\rho_{S S}$, (b) long-spaced density image, $\rho_{L S}$, (c) quadrant-sector near neutron count rates, $n c p s$, (d) quadrant-sector far neutron count rates, $f c p s$ in counts per second (cps), (e) quadrant-sector neutron compensated porosity, $\phi_{N}$, (f) GR image, (g) PEF log, and (h) $2 \mathrm{MHz}$ phase-shift array propagation resistivity curves with layer designations. Layer properties are shown in grey stair plots. 


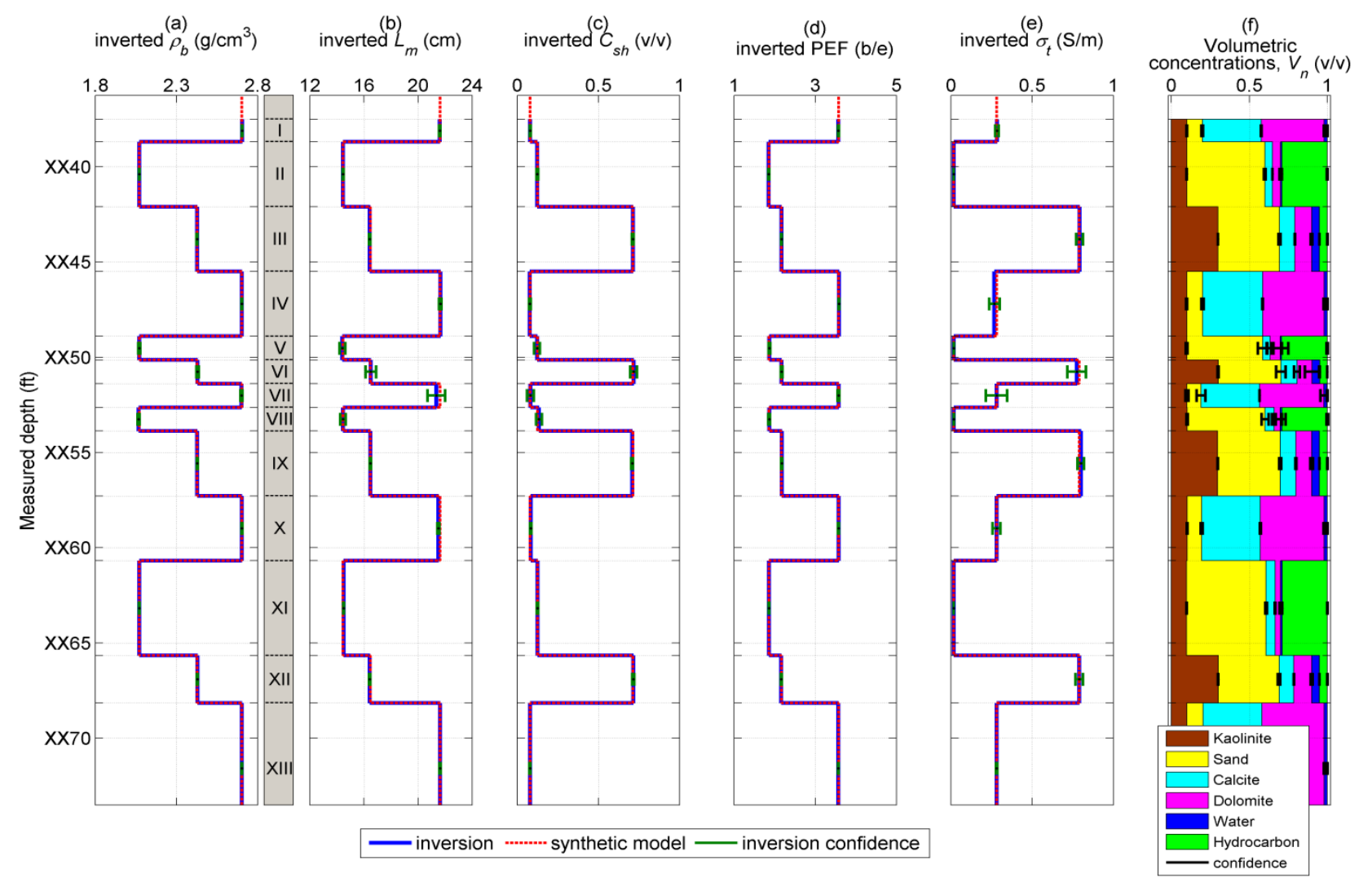

Figure 4.3: $\quad$ Layer-based inversion results for the synthetic example, showing inverted (a) bulk density, $\rho_{b}$ with layer designations, (b) migration length, $L_{m}$, (c) shale concentration, $C_{s h}$, (d) photoelectric factor, PEF, and (e) true conductivity, $\sigma_{t}$, in solid blue lines. True synthetic model properties are identified with dashed red lines, respectively, while $95 \%$ inversion confidence intervals are shown with solid green error bars, respectively. (f) Cumulative plots of compositional volumetric concentrations, $V_{n}$ obtained from SNUPAR-based solver and Archie's saturation model. Confidence intervals or uncertainties in compositional volumes, per layer, are shown with black error bars. 

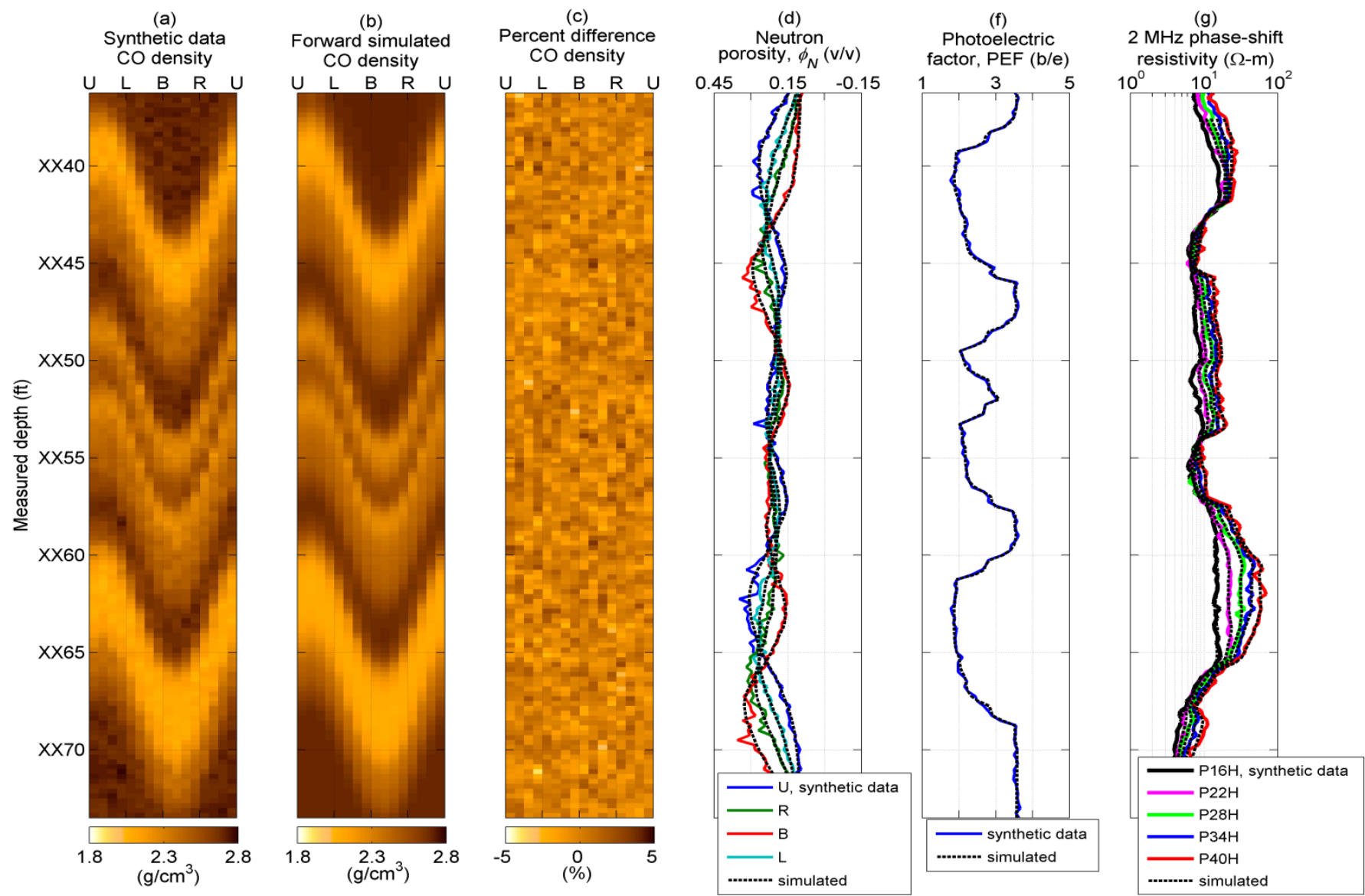

Figure 4.4: Comparison of synthetic data and numerically simulated measurements from inverted compositions. (a) Synthetic compensated density data, $\rho_{C O}$, (b) forward simulated compensated density from inverted compositions, (c) percent difference between synthetic and forward simulated $\rho_{C O}$ data, (d) quadrant-sector neutron porosity, $\phi_{N} \operatorname{logs}$, (e) PEF logs, and (f) $2 \mathrm{MHz}$ phase-shift array resistivity curves. Forward simulated curves are identified with dashed black lines. 

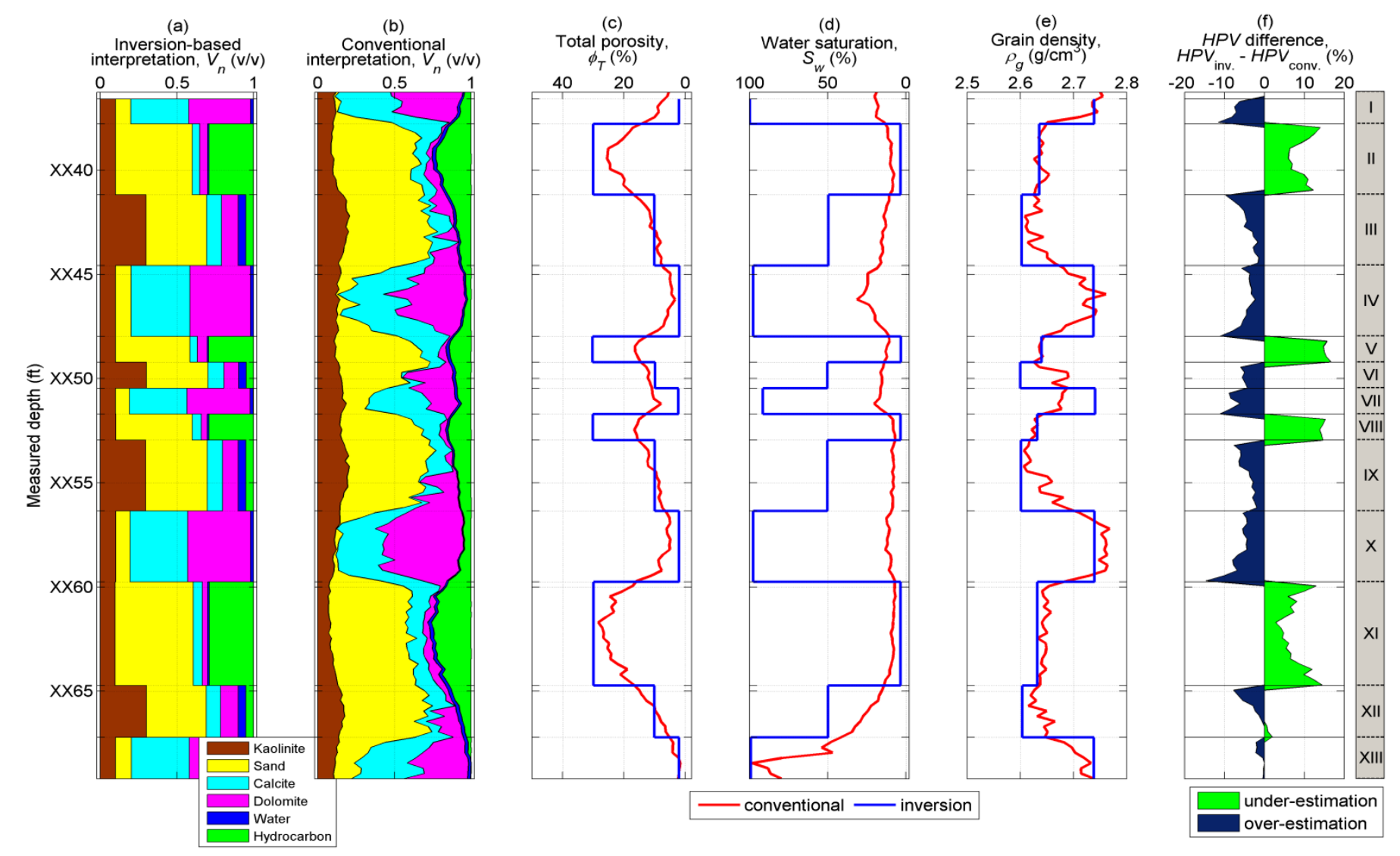

Figure 4.5: Comparison of inversion-based and conventional interpretation of synthetic LWD nuclear and resistivity measurements acquired in $82^{\circ}$ up-dip well. Cumulative plots of compositional volumetric concentrations, $V_{n}$, obtained from SNUPAR-based solver and Archie's saturation model for (a) inversion-based interpretation, and (b) conventional interpretation. Comparison of calculated (c) total porosity, $\phi_{T}$, (d) water saturation, $S_{w}$, and (e) grain density, $\rho_{g}$. (f) Difference in hydrocarbon pore volume, $H P V$ between inversion-based and conventional interpretation. 


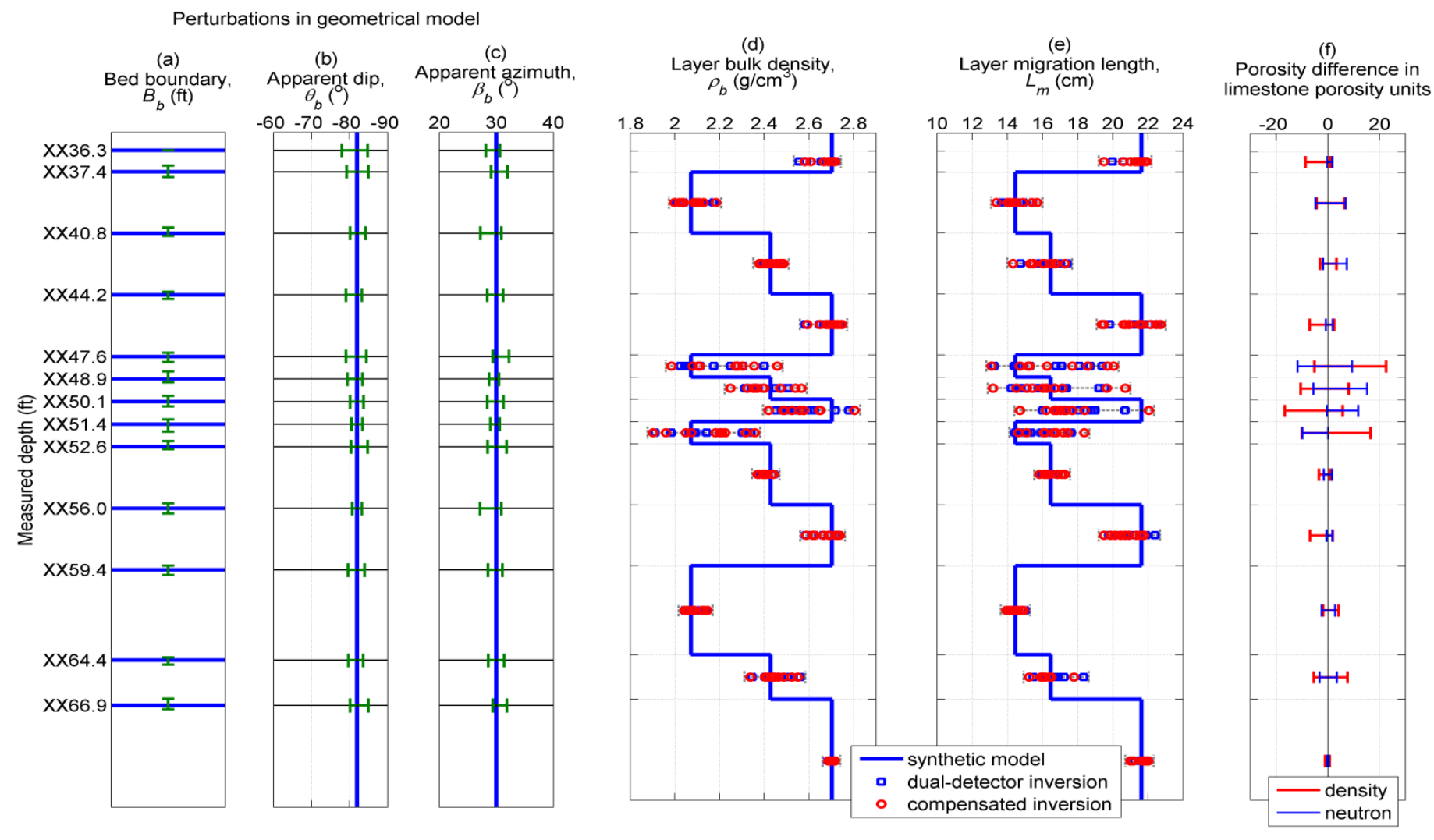

Figure 4.6: Effects of bed-geometry uncertainty on layer-based inversion of dual-detector and compensated density and neutron porosity measurements. Descriptions of uncertainty in the geometrical model: From left to right, panels show perturbations in (a) bed boundary location, $B_{b}$, (b) apparent bed dip, $\theta_{b}$, and (c) apparent bed azimuth, $\beta_{b}$. (d) Bulk density, $\rho_{b}$ and (e) migration length, $L_{m}$ from inversion of density and neutron measurements, respectively. Solid blue lines identify true layer-by-layer properties, while blue square points and red circle points identify results obtained from the inversion of dual-detector and compensated measurements, respectively. (f) Porosity difference, in limestone porosity units, due to bed-geometry uncertainty. 

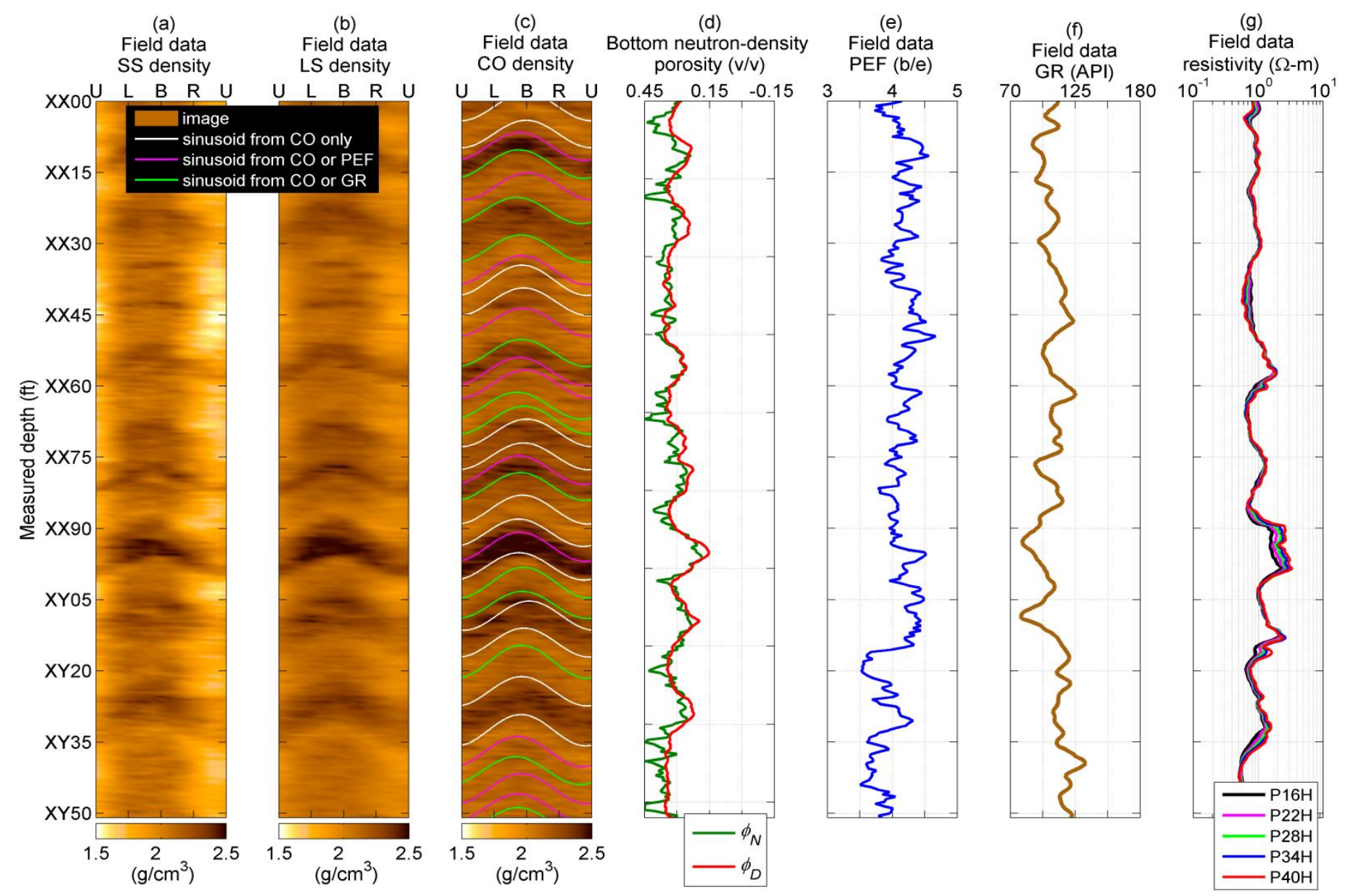

Figure 4.7: Field case example of LWD nuclear and resistivity measurements in a HA interval. (a) Short-spaced density image, $\rho_{S S},(\mathrm{~b})$ long-spaced density image, $\rho_{L S}$, (c) compensated density image, $\rho_{C O}$, showing consolidated gamma borehole sinusoids, (d) bottom sector neutron, $\phi_{N}$, and density, $\phi_{D}$, porosity logs in limestone scale, (e) PEF log, (f) GR log, and (g) 2 MHz phase-shift resistivity curves. 


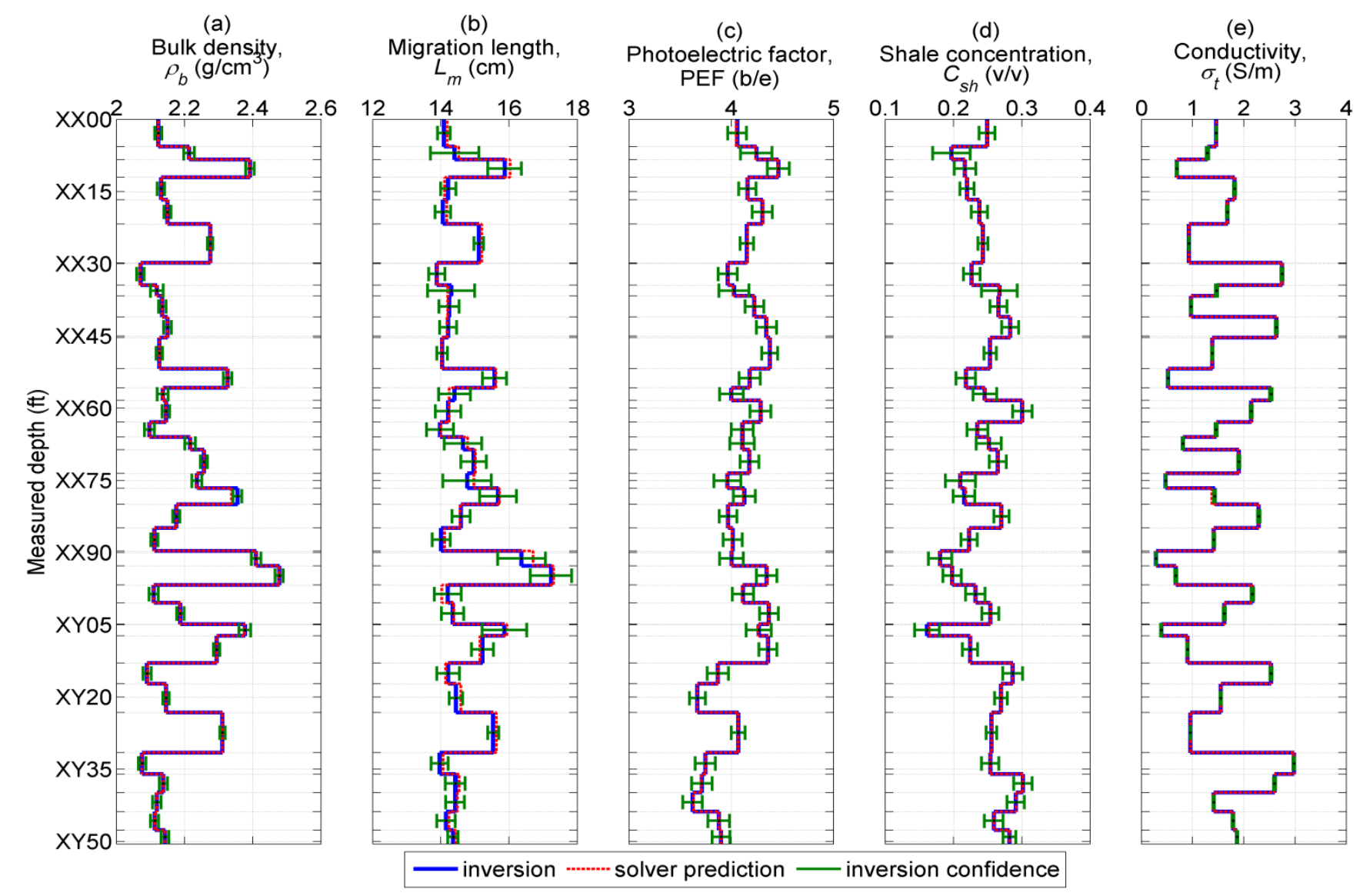

Figure 4.8: Layer-based inversion results of HA field example, showing inverted (a) bulk density, $\rho_{b}$, (b) migration length, $L_{m}$, (c) photoelectric factor, PEF, (d) shale concentration, $C_{s h}$, and (e) true conductivity, $\sigma_{t}$, in solid blue lines. Predicted properties of SNUPAR-based solver and Poupon-Leveaux saturation model for output compositional volumes are identified with dashed red lines; $95 \%$ inversion confidence intervals are identified with green error bars. 

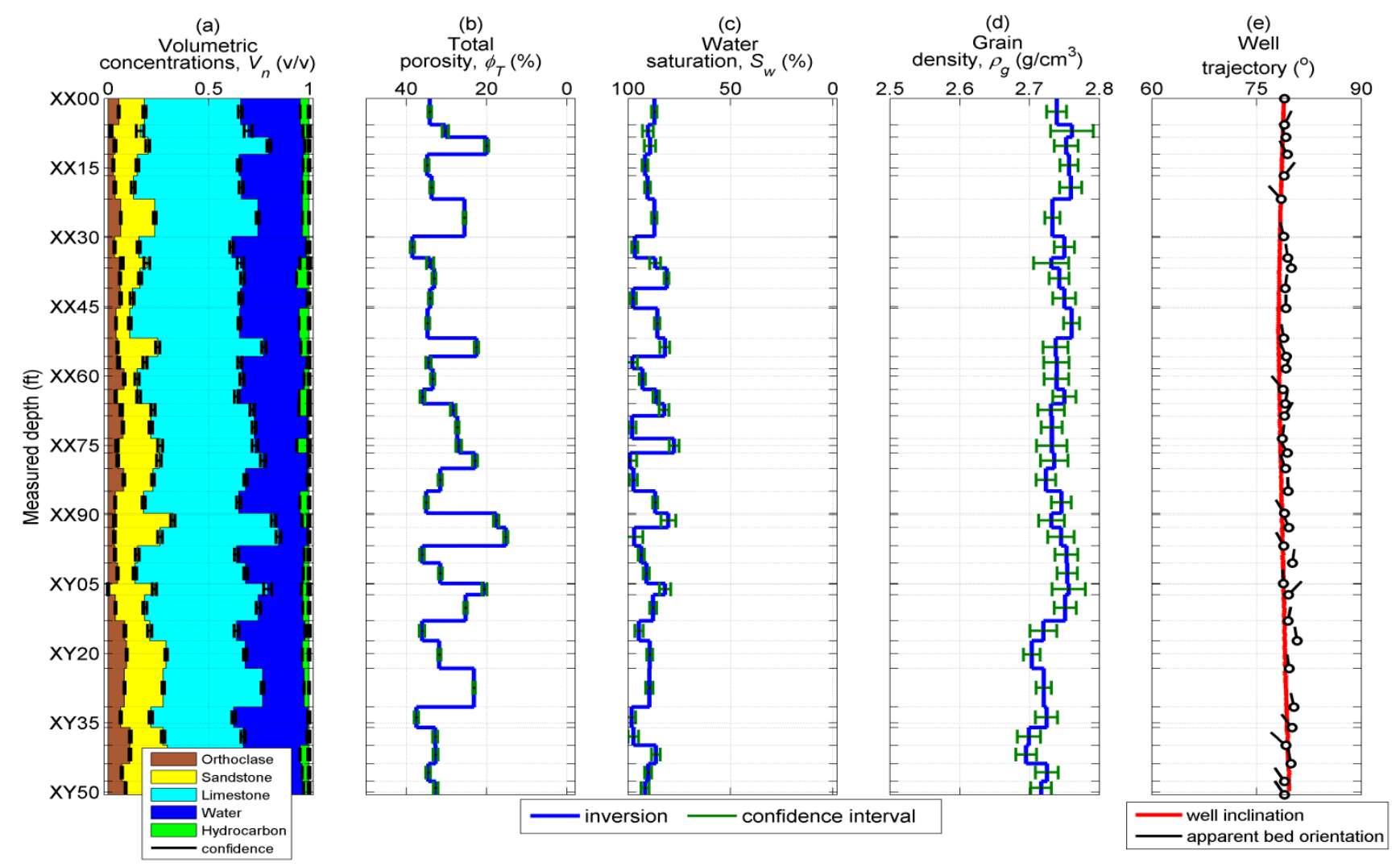

Figure 4.9: Calculated petrophysical properties from inversion results of HA field example. (a) Cumulative plots of compositional volumetric concentrations, $V_{n}$, obtained from SNUPAR-based solver and Poupon-Leveaux saturation model. Confidence intervals or uncertainties in compositional volumes, per layer, are identified with black error bars. Calculated (b) total porosity, $\phi_{T}$, (c) water saturation, $S_{w}$, and (d) grain density, $\rho_{g}$. The $95 \%$ inversion confidence intervals are identified with solid green error bars. (e) Well trajectory across HA interval is identified with a solid red line and apparent bed orientation is identified with tadpole-like points, i.e., bed dip and azimuth. 
Field data CO density

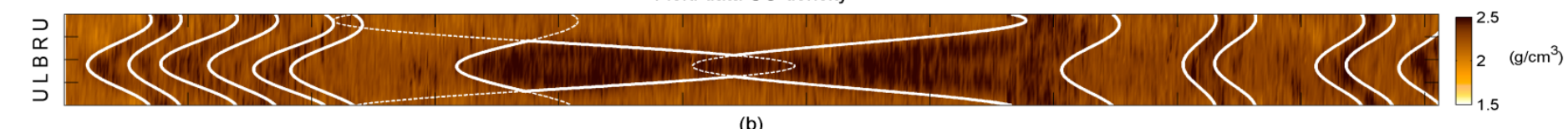

Field data $2 \mathrm{MHz}$ phase resistivity
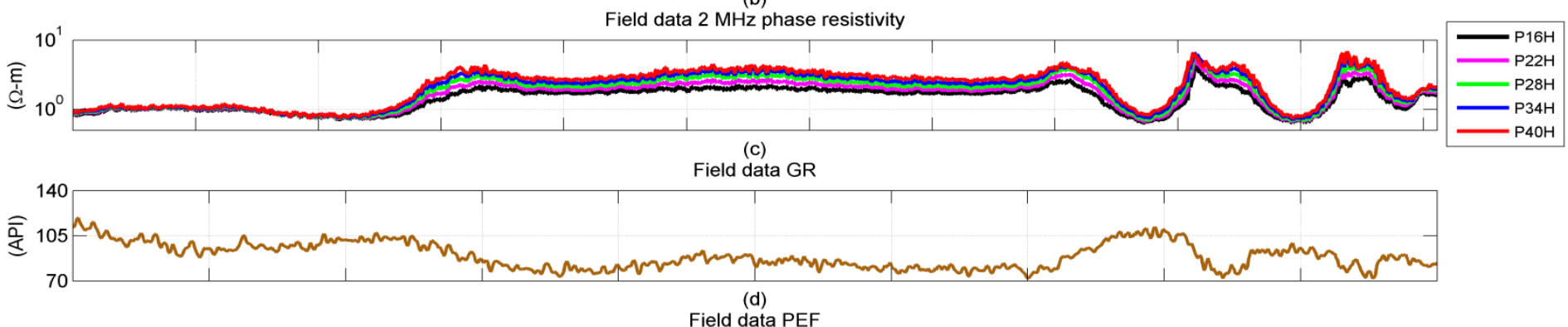

Field data PEF

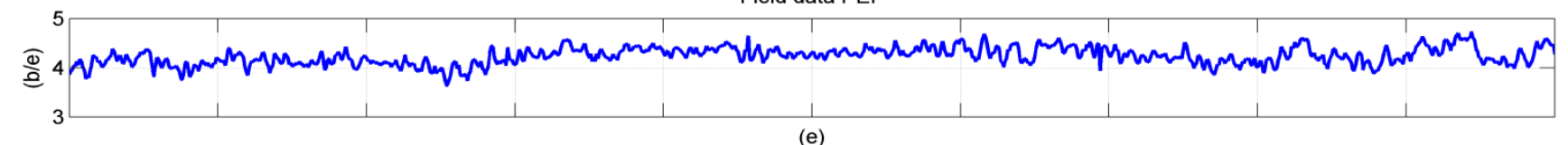

Bottom neutron-density porosity

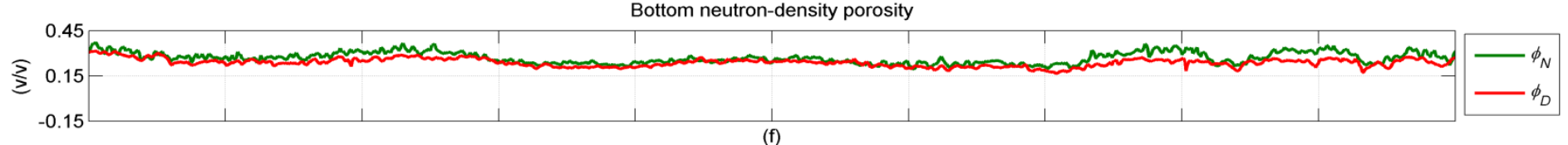

Schematic of layer-based geometry

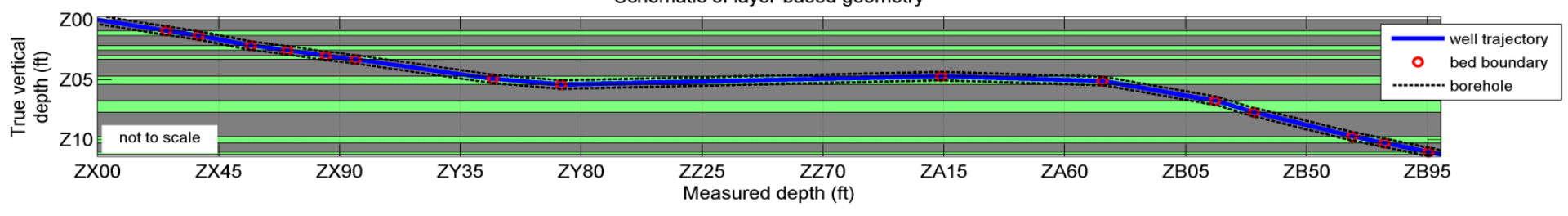

Figure 4.10: Field case example of LWD nuclear and resistivity measurements in a HZ interval. (a) Compensated density image, $\rho_{C O}$, showing gamma borehole sinusoids, (b) $2 \mathrm{MHz}$ phase-shift resistivity curves, (c) GR log, (d) PEF $\log$, (e) bottom sector neutron, $\phi_{N}$, and density, $\phi_{D}$, porosity logs in limestone scale, and (f) 2D curtain section diagram of layer-based geometry and well trajectory (not to scale). 


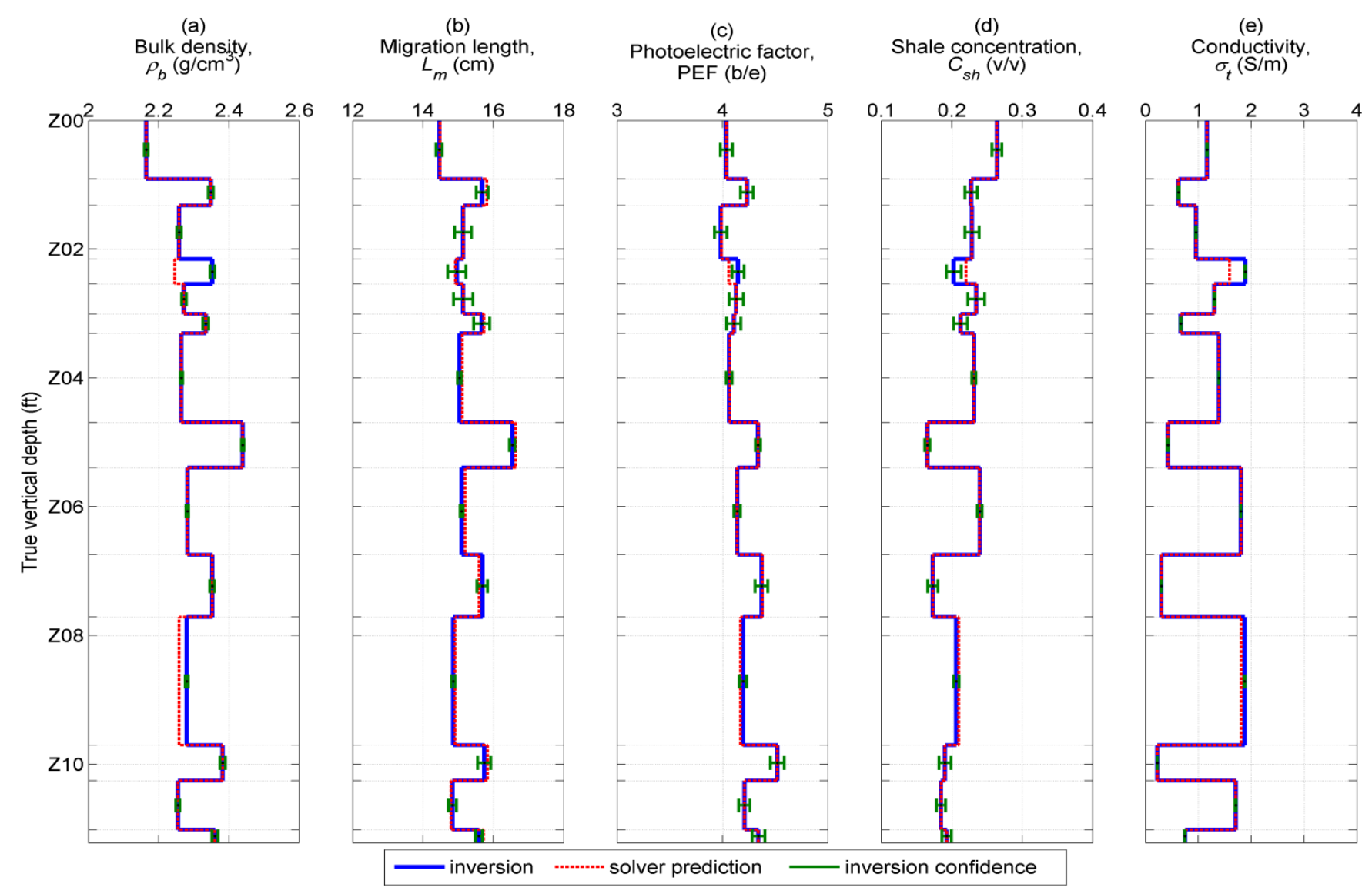

Figure 4.11: Layer-based inversion results of $\mathrm{HZ}$ field example, showing inverted (a) bulk density, $\rho_{b}$, (b) migration length, $L_{m}$, (c) photoelectric factor, PEF, (d) shale concentration, $C_{s h}$, and (e) true conductivity, $\sigma_{t}$, in solid blue lines. Predicted properties of SNUPAR-based solver and Poupon-Leveaux saturation model for output compositional volumes are identified with dashed red lines; $95 \%$ inversion confidence intervals are identified with green error bars. 


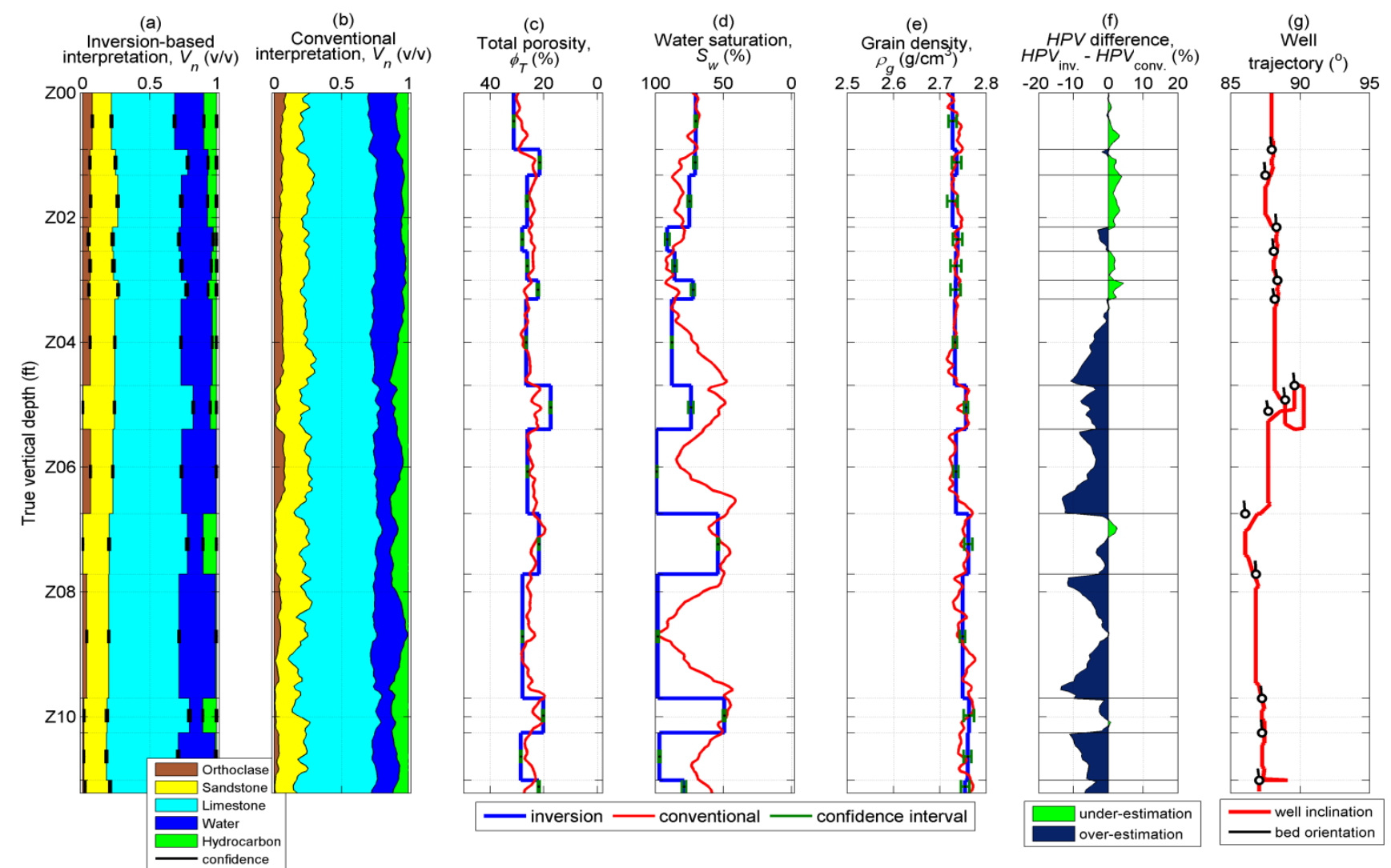

Figure 4.12: Comparison of inversion-based and conventional interpretation results of HZ field example. Cumulative plots of compositional volumetric concentrations, $V_{n}$, obtained from SNUPAR-based solver and Poupon-Leveaux saturation model for (a) inversion-based interpretation, and (b) conventional interpretation. Comparison of (c) total porosity, $\phi_{T}$, (d) water saturation, $S_{w}$, and (e) grain density, $\rho_{g}$. (f) Difference in hydrocarbon pore volume, $H P V$, between inversion-based and conventional interpretation. $(\mathrm{g})$ Well trajectory across $\mathrm{HZ}$ interval is identified with a solid red line and apparent bed orientation is identified with tadpole-like points, i.e., bed dip and azimuth. 


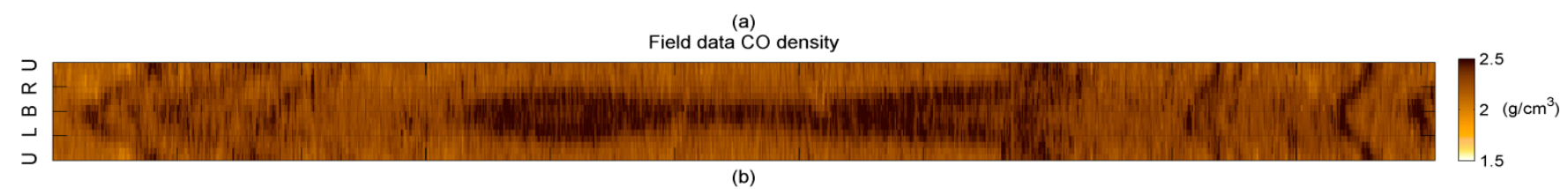

Forward simulated CO density

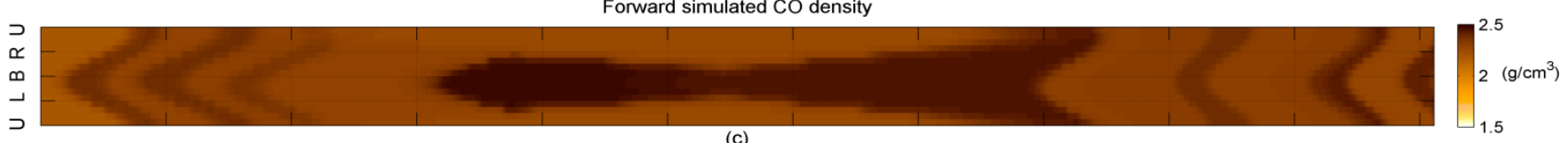

Field and forward simulated $\phi_{N}$

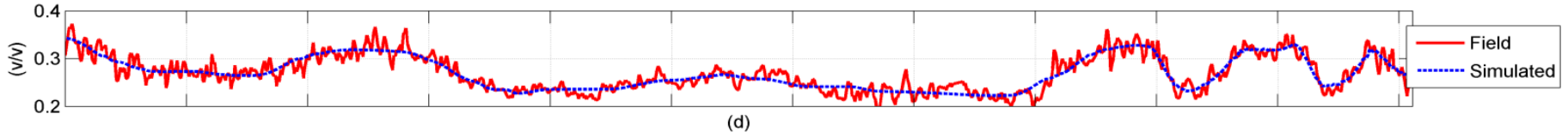

Field and forward simulated GR

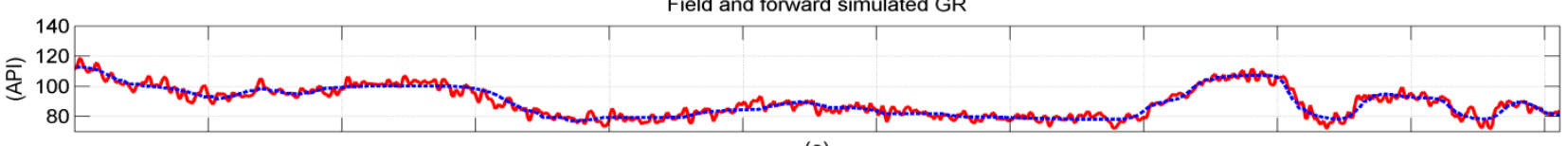

Field and forward simulated PEF

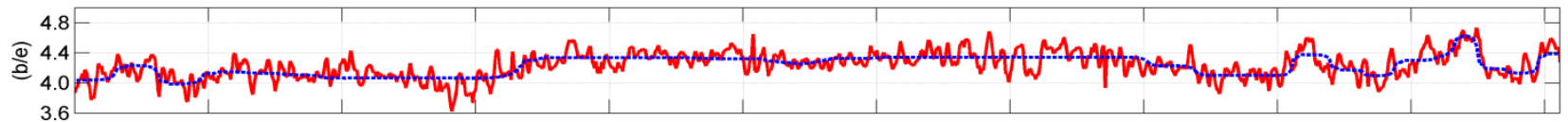

Field and forward simulated $2 \stackrel{(\mathfrak{f})}{\mathrm{MHz}}$ phase-shift resistivity curves

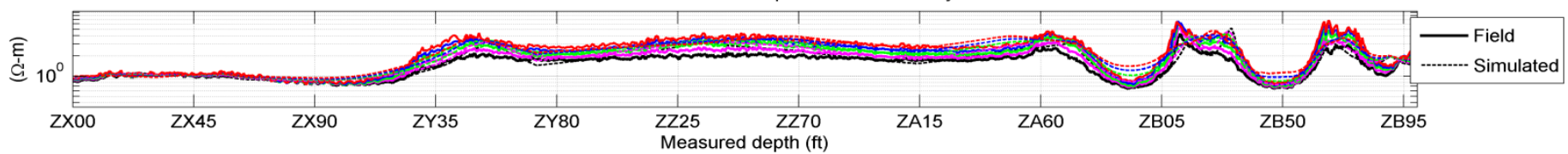

Figure 4.13: Qualitative comparison of forward simulated measurements, from inverted compositions, and HZ field data. (a) Field compensated density data, $\rho_{C O}$, and (b) forward simulated compensated density from inverted compositions. Field and forward simulated (c) neutron porosity, $\phi_{N},(d)$ GR, and (e) PEF logs. Field and forward simulated logs are identified with solid red and dashed blue lines, respectively. (f) $2 \mathrm{MHz}$ phase-shift array resistivity curves, field and forward simulated curves are identified with solid and dashed colored lines, respectively. 


\section{Chapter 5: Interpretation of Porosity and Fluid Constituents from Well Logs Using an Interactive Neutron-Density Matrix Scale}

Neutron and density logs are important borehole measurements for estimating reservoir capacity and inferring saturating fluids. The neutron log, measuring hydrogen index (HI), is commonly expressed in apparent water-filled porosity units assuming a constant matrix lithology whereby it is not always representative of actual pore fluid. By contrast, a lithology-independent porosity calculation from nuclear magnetic resonance (NMR) or/and core measurements provides reliable evaluations of reservoir capacity. In practice, not all wells include core or NMR measurements. This chapter introduces an interpretation workflow wherein formation porosity and hydrocarbon constituents can be estimated from density and neutron logs using an interactive, variable matrix scale specifically suited for the pre-calculated matrix density.

Firstly, I estimate matrix components from combinations of nuclear logs (photoelectric, natural gamma ray, neutron, and density) using Schlumberger's Nuclear Parameter calculator (SNUPAR) as a matrix compositional solver while assuming freshwater-filled formations. The combined effects of grain density, volumetric concentration of shale, matrix hydrogen, and neutron lithology units define an interactive matrix scale for correction of neutron porosity. Under updated matrix conditions, the resulting neutron-density crossover can only be attributed to pore volume and saturating fluid effects. Secondly, porosity, connate-water saturation, and hydrocarbon density are calculated from the discrepancy between corrected neutron and density logs using SNUPAR and Archie's water saturation equation, thereby eliminating the assumption of fresh-water saturation. With matrix effects eliminated from the neutron-density overlay, gas- or light-oil-saturated formations exhibiting the characteristic gas neutron-density 
crossover become representative of saturating hydrocarbons. This behavior gives a clear qualitative distinction between hydrocarbon- saturated and non-viable depth zones.

\section{$5.1 \quad$ INTRODUCTION}

Porosity calculated from neutron and density measurements is still the most reliable estimate of reservoir porous space from well-log analysis. In complex lithologies, inadequate characterization of the matrix could yield inaccurate porosity and saturation estimates. The petrophysical effects of lithology, saturating fluid, and borehole conditions on nuclear logs were exhaustively discussed by Ellis et al. (2007). Using departure curves from log interpretation charts (Schlumberger, 2009), corrections are applied such that interpreted properties are representative of the formation only. Extensive studies and publications on neutron and density logs, being ubiquitous for porosity and hydrocarbon estimation, can be found in the literature.

Historically, total porosity, $\phi_{t}$, in gas-bearing formations is approximated with the formula (Gaymard et al., 1968)

$$
\phi_{t}^{2} \approx \frac{\phi_{N}^{2}+\phi_{D}^{2}}{2},
$$

where $\phi_{D}$ and $\phi_{N}$ are density and neutron apparent porosities, respectively. Mao (2001) studied the correlation characteristics of $\phi_{D}$ and $\phi_{N}$ for identification of oil- and gassaturated zones. Spears (2006) applied lithofacies-based porosity corrections derived from neutron-density cross-plots for $\phi_{t}$ calculations in geologic and reservoir models. Fertl et al. (1971) extended Gaymard et al. (1968) formulations for calculation of hydrocarbon density, $\rho_{h c}$, and detection of oil- and gas-bearing intervals in shaly sands. 
The neutron-density overlay technique relies on the difference between apparent porosities, on a pre-defined matrix scale, for inferring hydrocarbon saturation $\left(S_{h c}\right)$, $\phi_{t}$, and $\rho_{h c}$. Several petrophysical factors adversely affect the reliability of the overlay technique. For example, gas detection is challenging in shaly sands or shale gas reservoirs due to opposite effects of shale-hydroxyls and gas density in overlay characteristics. Similarly, in oil-saturated or invaded gas zones, the decreased difference between neutron and density apparent porosities masks the characteristics of lighthydrocarbon crossovers (Mao, 2001). Consequently, application of the overlay technique requires implementation of a suitable matrix correction.

In this chapter, I estimate $S_{h c}, \phi_{t}$, and $\rho_{h c}$ using an interactive interpretation workflow based on the neutron-density overlay technique, with explicit consideration of neutron matrix scale and shale content. The interpretation workflow improves reliability of the overlay technique in the presence of arbitrary lithology and fluid effects. These effects and their influence on neutron and density apparent porosities, along with the typical interpretation thought processes, are described with a synthetic example of known lithology and fluid constituents. Additionally, the interactive matrix scale method is applied to field examples of varying geology and lithology, namely carbonate, siliciclastic, and shale reservoirs, where porosity and fluid-saturation estimates are compared to laboratory core measurements. The calculated $\rho_{h c}$ enables differentiation between gas and oil-saturated intervals when $\rho_{h c}<0.25 \mathrm{~g} / \mathrm{cm}^{3}$ and $\rho_{h c} \geq 0.25 \mathrm{~g} / \mathrm{cm}^{3}$, respectively, for simplified interpretation. Hence, the interactive analysis method is implemented for qualitative identification of fluid zones, fluid contacts, and reservoir compartments. 


\subsection{INTERPRETATION OF APPARENT POROSITY}

The porosity value associated with neutron logs is inherently apparent for given matrix and fluid units. On the other hand, compensated bulk density measurements bear no apparentness until density porosity is calculated with constant values of matrix and fluid properties. This is a significant difference between density and neutron logs.

\subsubsection{Density Apparent Porosity}

The bulk density measurement, $\rho_{b}$, principally responds to formation electron density such that

$$
\rho_{b}=\sum_{i=1}^{M}\left(1.0704 \rho_{e, i}-0.188\right) V_{i},
$$

where $\rho_{e, i}$ and $V_{i}$ are electron density and volume fraction, respectively, of the $i$-th fluid/matrix component up to $M$ components. In hydrocarbon-bearing formations, $\phi_{t}$ can be directly calculated from density $\operatorname{logs}$ if and only if matrix density, $\rho_{m}$, and fluid density, $\rho_{f}$, are known precisely. Otherwise, density apparent porosity, $\phi_{D}$, is obtained using

$$
\phi_{D}=\frac{\rho_{b}-\rho_{m}}{\rho_{f}-\rho_{m}}=\phi_{t}+\Delta \phi_{D},
$$

where $\rho_{m}$ and $\rho_{f}$ are assumed matrix and fluid densities, respectively, e.g. limestone matrix of $2.71 \mathrm{~g} / \mathrm{cm}^{3}$ and fresh-water of $1 \mathrm{~g} / \mathrm{cm}^{3}$. The porosity departure, $\Delta \phi_{D}$, due to $\rho_{m}$ and $\rho_{f}$ assumptions (Ellis et al., 2007) is both qualitatively and quantitatively intuitive such that 


$$
\Delta \phi_{D} \approx \frac{\rho_{b}-\rho_{m}}{\left(\rho_{f}-\rho_{m}\right)^{2}}\left(\Delta \rho_{m}-\Delta \rho_{f}\right)-\frac{1}{\rho_{f}-\rho_{m}} \Delta \rho_{m}
$$

where $\Delta \rho_{m}$ and $\Delta \rho_{f}$ are differences in matrix and fluid densities, respectively, between assumed and true properties.

\subsubsection{Neutron Apparent Porosity}

The neutron $\log$ is an apparent porosity measurement, given that it refers to an equivalent hydrogen index response in water-filled lithology units, usually limestone. Limestone unit implies an equivalent response of water-filled limestone formation where the pore volume equals that of the neutron log. As shown by Gaymard et al. (1968), the environmentally-corrected neutron porosity $\log , \phi_{N}$, across invaded formations can be expressed as

$$
\phi_{N}=\frac{1}{H I_{m f}} \sum_{i=1}^{M} H I_{i} V_{i}=\phi_{t}+\Delta \phi_{N}
$$

where $H I$ is hydrogen index, subscript $m f$ identifies mud filtrate, and $\Delta \phi_{N}$ is porosity departure due to neutron apparent porosity measurement. Equation 5.5 implies that the neutron response is a superposition of the volumetric contributions of component hydrogen concentrations. The apparentness in $\Delta \phi_{N}$ is determined by the neutron porosity unit, usually water-filled limestone. For example, quartz, calcite, and dolomite blocks yield $\Delta \phi_{N}$ of $-2,0$, and 0.5 limestone pu (porosity units), respectively. This matrix effect is qualitatively intuitive because quartz and dolomite have lower and higher matrix densities, respectively, than limestone. On the other hand, unlike $\phi_{D}$, the matrix effect is quantitatively obscure and cannot be calculated directly from equations 5.3 or 5.4. This 
effect is exacerbated in complex mixtures of various lithologies. Similarly, a gassaturated limestone formation yields negative $\Delta \phi_{N}$ because the hydrogen index of gas is typically lower than that of water.

Hence, a physically intuitive parameter representation of neutron porosity responses is necessary. Using neutron characteristic lengths, specifically migration length, $L_{m}$, a calibration of $L_{m}$-to-neutron porosity is used to quantify matrix and lithology effects (Ellis et al., 2007).

\subsection{Neutron Parameter Model}

The Schlumberger Nuclear Parameter calculator (SNUPAR, McKeon et al., 1989) calculates nuclear properties such as $L_{m}, H I$, photoelectric factor (PEF), capture cross section, $\Sigma$, etc., for any given mixture of rocks and fluids. In this chapter, I implement $L_{m}$ for property characterization of wireline neutron porosity responses, typically with an AmBe (americium-beryllium) neutron source. It follows that equation 5.5 can be rewritten as

$$
\phi_{N}=g_{l}\left(\frac{1}{L_{m}}\right),
$$

where $g_{l}$ represents the $L_{m}$-to-porosity calibration function in limestone water-filled units; $g_{l}$ is obtained by fitting a polynomial function to the inverse of SNUPARcalculated $L_{m}$ and limestone pore volume. Figure 5.1 shows $g_{s}, g_{l}$, and $g_{d}$ water-filled calibration functions for sandstone, limestone, and dolomite units, respectively. Additionally, the SNUPAR-calculated Compensated Neutron Tool (CNT) thermal porosity response, shown in dashed blue, agrees well with $g_{l}$. Unless otherwise stated, 
neutron porosity logs in this chapter are expressed in limestone matrix units, where $g_{l}^{-1}$ and $g_{l}$ are used to convert neutron porosity to $L_{m}$ logs, and vice versa, respectively.

Furthermore, I implement a SNUPAR-based compositional solver (Appendix B, section B.2) for estimation of mineral and fluid concentrations from nuclear logs. The solver uses nonlinear minimization of a constrained-error, quadratic cost function between SNUPAR-predicted properties and nuclear logs $\left(\rho_{b}, \phi_{N}, \mathrm{PEF}\right)$ for estimation of mineral and fluid volumetric fractions. Additionally, volumetric concentration of shale, $V_{s h}$, and water saturation, $S_{w}$, are calculated using linear scaling of the gamma ray (GR) $\log$ and Archie's equation, respectively.

\subsection{LITHOLOGY EFFECTS}

\subsubsection{Matrix Effect}

Equation 5.4 describes the sensitivity of $\phi_{D}$ such that the matrix effect in water zones, i.e., when $\Delta \rho_{f}=0$ and $\rho_{f}=\rho_{c w}$ (connate-water density), is given by

$$
\Delta \phi_{D} \approx\left[\frac{\rho_{b}-\rho_{m}}{\left(\rho_{f}-\rho_{m}\right)^{2}}-\frac{1}{\rho_{f}-\rho_{m}}\right] \Delta \rho_{m} .
$$

In the neutron-density overlay technique, water-saturated zones are expected to overlap only if the matrix scale for both density and neutron corresponds to the precise formation lithology. Otherwise, the neutron-density matrix effect, $\Delta \phi_{\text {matrix }}=\Delta \phi_{N}-\Delta \phi_{D}$ (equations 5.3 and 5.5), depends on $\Delta \rho_{m}$ and the neutron response, $\phi_{N}$, of the matrix. Unlike $\phi_{D}$ in equation 5.3, $\phi_{N}$ of the matrix is not quantitatively intuitive, and is only obtained from equation 5.6 by converting SNUPAR-calculated $L_{m}$ to neutron porosity. Qualitatively, 
with a limestone matrix scale in water zones, $\Delta \phi_{\text {matrix }}<0$ across sandstone and $\Delta \phi_{\text {matrix }}>$ 0 across dolomite.

\subsubsection{Shale-Hydroxyl or Matrix-Hydrogen Effect}

Typically, shales consist of clay minerals with high hydroxyl $\left(\mathrm{OH}^{-}\right)$content such that $\phi_{N}>\phi_{D}$. The shale-hydroxyl effect, $\Delta \phi_{N s h}$, can be approximated from equation 5.6 using the expression

$$
\Delta \phi_{N s h}=V_{s h} g_{l}\left(\frac{1}{L_{m s h}}\right),
$$

where $L_{m s h}$ is SNUPAR-calculated migration length in shale. For example, $L_{m s h}$ is approximately $15.35 \mathrm{~cm}$ for illite of density $2.78 \mathrm{~g} / \mathrm{cm}^{3}$, whereby $\Delta \phi_{N s h}$ for pure illite (refer to Figure 5.1), i.e., $V_{s h}=1$, corresponds to 0.156 . In unconventional reservoirs with organic-rich kerogen matrix (Passey et al., 1990), the neutron porosity response increases due to high hydrogen content of organic matter. The SNUPAR-calculated HI of kerogen could be as high as 0.8 , depending on both hydrogen-carbon ratio and kerogen density. Accordingly, equation 5.8 quantifies the matrix-hydrogen effect where $V_{s h}$ and $L_{m s h}$ become $V_{k e r}$ (volume fraction of kerogen) and $L_{m k e r}$ (SNUPAR-calculated migration length of kerogen matrix), respectively.

It then follows the total matrix effect on neutron porosity logs is an addition of $\Delta \phi_{\text {matrix }}$ and $\Delta \phi_{N s h}$, i.e., interactive porosity departures due to apparent limestone matrix scale (calculated from SNUPAR in fresh-water-filled assumptions, equations 5.6, and 5.7) and shale-hydroxyl or matrix-hydrogen effect (equation 5.8). The corrected or rescaled neutron apparent porosity is given by 


$$
\phi_{N c o r r}=\phi_{N}-\left(\Delta \phi_{\text {matrix }}+\Delta \phi_{N s h}\right) \text {. }
$$

\subsection{FLUID AND HydRocarbon SATURATION EFFECTS}

Given equations 5.3, 5.5, 5.6, and 5.9, fluid and saturation effects on re-scaled neutron apparent porosity can be written as

$$
\phi_{N c o r r}=\phi_{t}\left(1+S_{h c} \delta_{\Sigma}\right) \text {, }
$$

where $\delta_{\Sigma}$ is the difference in neutron response between hydrocarbon-saturated and watersaturated formations. Several forms of equation 5.10 are given in Gaymard et al. (1968), Mao (2001), and Quintero et al. (1998). Gaymard et al. (1968) characterized $\delta_{\Sigma}$ across invaded formations as the relative difference in $H I$ between residual hydrocarbon and mud-filtrate, i.e.

$$
\delta_{\Sigma}=\left(H I_{h c}-H I_{m f}\right) / H I_{m f},
$$

where subscript $h c$ identifies hydrocarbon. For gas-saturated formations at reservoir conditions one has

$$
H I_{g} \approx 9 \rho_{g}\left[0.15+0.2\left(0.9-\rho_{g}\right)^{2}\right] \text {, }
$$

where subscript $g$ describes gas. Equation 5.12 is replicated in SNUPAR for $\rho_{h c}=\rho_{g}<$ $0.25 \mathrm{~g} / \mathrm{cm}^{3}$, while a SNUPAR-derived functional relationship is obtained for oil $\left(\mathrm{C}_{\mathrm{n}} \mathrm{H}_{2 \mathrm{n}+2}\right)$ when $\rho_{h c}>0.25 \mathrm{~g} / \mathrm{cm}^{3}$. Estimation of $S_{h c}, \phi_{t}$, and $\rho_{h c}$ thus requires solving equations 5.2, $5.10,5.11,5.12$, and inclusion of a water saturation model, e.g. Archie's equation, 


$$
R_{t}=\frac{a R_{w}}{\phi_{t}^{m}\left(1-S_{h c}\right)^{n}},
$$

where $R_{t}$ is resistivity $\log , R_{w}$ is connate-water or mud-filtrate resistivity, $a$ is Archie's factor, $m$ is porosity exponent, and $n$ is saturation exponent. It follows that $\delta_{\Sigma}=0$ corresponds to a water or deeply invaded zone. Consequently, the magnitudes of $\delta_{\Sigma}$ and $\rho_{h c}$ dictate the hydrocarbon type, i.e., oil or gas.

\subsection{Interactive ANalysis OF Matrix ANd Fluid EFfects}

Well-log interpretation involves thought processes and considerations that evaluate formation rock compositions and their saturating fluids. This chapter introduces an interpretation practice or interactive analysis workflow that combines petrophysical effects due to apparent matrix scale and hydrocarbon saturation. Using a synthetic example of a layered earth model, where well logs are simulated with UTAPWeLS (The University of Texas at Austin Petrophysical and Well-Log Simulator, Voss et al., 2009), I describe the estimation of $\phi_{t}, \rho_{h c}$, and $S_{h c}$ using the interactive interpretation workflow.

\subsubsection{Interpretation Workflow}

The first part of the interpretation involves matrix compositional interpretation from $\rho_{b}$, PEF, and GR logs using the SNUPAR-based solver (Appendix B, section B.2) under the assumption of fresh-water-filled saturation. I assume fresh-water-filled formations for two reasons: (1) the environmentally-corrected $\phi_{N}$ is typically referenced on fresh-water-filled units, and (2) to independently characterize matrix effects for estimation of $\rho_{m}$ given that formation fluids have negligible or no effect on PEF and GR $\operatorname{logs}$. 
Using the estimated $\rho_{m}$ from the matrix solver and equation 5.3, I calculate density apparent porosity under the fresh-water-filled assumption, $\phi_{D w f}$. Accordingly, neutron apparent porosity under the fresh-water-filled assumption, $\phi_{N w f}$, is obtained by converting the predicted $L_{m}$ from the matrix solver to neutron porosity. It follows from equations 5.8 and 5.9 that $\Delta \phi_{\text {matrix }}+\Delta \phi_{N s h}=\phi_{N w f}-\phi_{D w f}$, i.e. the interactive neutron-density lithology effect in limestone porosity scale, where $V_{s h}$ is calculated assuming linear scaling of the GR log. I then calculate the corrected neutron apparent porosity, $\phi_{\text {Ncorr }}$ from equation 5.9 for re-scaling with $\phi_{D}$. At this point, the overlay characteristics of $\phi_{N w f}$ and $\phi_{D w f}$ are solely due to porosity effects, while the overlay of $\phi_{N c o r r}$ and $\phi_{D}$ is due to hydrocarbon pore volume.

The second part of the interpretation involves implementing the SNUPAR-based solver for hydrocarbon characterization. In this step, equations 5.2, 5.10, 5.11, 5.12, and 5.13 are solved such that a SNUPAR-defined inherent relationship between $\delta_{\Sigma}$ and $\rho_{h c}$ is implemented in the analysis for estimation of $\rho_{h c}, S_{h c}$, and $\phi_{t}$. The functional relationship between $H I$ and $\rho_{h c}$ is derived from SNUPAR for oil $\left(\rho_{h c}>0.25 \mathrm{~g} / \mathrm{cm}^{3}\right)$ and gas $\left(\rho_{h c}<\right.$ $0.25 \mathrm{~g} / \mathrm{cm}^{3}$ ). Figure 5.2 summarizes the interpretation workflow of the interactive analysis.

Figure 5.2 summarizes the interpretation workflow where the "Matrix analysis" loop is interactive as rock components (e.g., quartz, dolomite, pyrite, etc.) are chosen to observe their effects on the calculated neutron-density matrix scale. Additionally, I compare estimated $\rho_{m}$ to core measurements wherever available and appraise the solver's prediction of PEF and GR measurements. Based on these comparisons, an interpretation decision or practice is made for the most representative formation rock components. Consequent to the "Fluid analysis" of Figure 5.2, the final interpreted results include 
total porosity, hydrocarbon density, water saturation, matrix/grain density, and formation rock components.

\subsubsection{Synthetic Example}

The interactive interpretation workflow is described for matrix and fluid effects

on density and neutron apparent porosities, using numerically simulated measurements across a synthetic and simplistic earth model. The model is designed to describe typical scenarios that present challenges in the practice of interpreting neutron and density logs.

Tables 5.1 and 5.2 describe the properties assumed for the synthetic earth model, while Figure 5.3 shows the simulated nuclear and resistivity logs. In Figure 5.4, I describe the interpretation results obtained with the interactive analysis workflow. Panels

$\mathrm{a}, \mathrm{f}, \mathrm{g}$, and h show that estimated $\rho_{m}, \phi_{t}, S_{w}$, and $\rho_{h c}$, respectively, using the interactive interpretation, agree well with model properties in Table 5.1. It is particularly significant that the calculated $\rho_{h c}$ in panel h distinguishes between gas and oil-saturated layers.

Layers I and IV consist of water-saturated shale of mixed orthoclase and illite clay, whereby $\Delta \phi_{s h}=0.155$ and shale density $\rho_{s h}=2.738 \mathrm{~g} / \mathrm{cm}^{3}$. After correction for shale-hydroxyl effects, the actual matrix crossover effect, due to the shale density greater than limestone density, is shaded in brown in panel b of Figure 5.4. On the other hand, layer V consists of gas-saturated shale with $20 \%$ water saturation (refer to Table 5.1), such that the gas crossover effect becomes accentuated after correction for shalehydroxyl effect. In this layer, because gas saturation and $V_{s h}$ impose opposite overlay characteristics, $\phi_{N}-\phi_{D}$ experiences a competition between gas and shale-hydroxyl effects. This behavior in neutron-density interpretation is especially common in logs acquired across shale gas formations. 
Layers II and III consist of gas- and oil-saturated limestone formations, respectively. The matrix effect is irrelevant in these layers because limestone is the reference scale for neutron-density overlay. This behavior is corroborated by the overlap of $\phi_{D w f}$ and $\phi_{N w f}$ in panel c of Figure 5.4. Hydrocarbons, in comparison to fresh water, reduce the neutron porosity response because of lower hydrogen index (equation 5.5). From equations 5.11 and 5.12, the hydrocarbon effect is dependent on $\rho_{h c}$ and is accentuated in gas-saturated layers when compared to oil-saturated layers. In panels d and e of Figure 5.4, layer III shows lower hydrocarbon effects and could be inadvertently interpreted as a water-filled layer. Consequently, the fluid solver incorporates the resistivity measurement, Archie's model (equation 5.13), equations 5.2, 5.10, 5.11, and 5.12 for an inclusive calculation of $\rho_{h c}, S_{h c}$, and $\phi_{t}$. Panel h shows that the estimated $\rho_{h c}$ reliably predicts gas and oil densities in gas- and oil-saturated layers II and III, respectively. In panel $\mathrm{f}$, the $\phi_{t}$ approximation using Gaymard-Poupon's formula (equation 5.1 ) is valid in layer II but inaccurate in shaly layers.

In layers VI and VII, for oil- and water-saturated dolomite, respectively, the overlay characteristics in panel b of Figure 5.4 indicate a matrix crossover. The matrix effect in panel d shows that $\Delta \phi_{\text {matrix }}=0.0072$ (i.e., $0.72 \mathrm{pu}$ ) for $\Delta \phi_{s h}=0$. By comparison, SNUPAR-calculated CNT response yields apparent thermal neutron porosity of $0.5 \mathrm{pu}$ in dolomite of $0 \%$ pore volume.

\subsection{Field EXAMPLES OF APPLICATION}

In this section of the chapter, the interactive interpretation workflow is implemented for estimation of $\rho_{m}, \phi_{t}, S_{w}$, and $\rho_{h c}$ in two field examples: (I) gas-bearing carbonate field of dolomite lithology where $\rho_{m}>2.71 \mathrm{~g} / \mathrm{cm}^{3}$, and (II) oil-bearing shale formation where $\rho_{m}<2.71 \mathrm{~g} / \mathrm{cm}^{3}$. 


\subsubsection{Field Example I, Gas-Bearing Carbonate}

This field example consists of conventional wireline nuclear and dual induction resistivity logs acquired across a gas-producing dolomite reservoir. Additionally, the well includes routine core measurements. Due to low reservoir pressure, deep mud-filtrate invasion affects the nuclear logs and even the deep resistivity log, such that log-derived $S_{h c}$ is considerably lower than in-situ $S_{h c}$ for water-base mud (Xu et al., 2012). Table 5.3 summarizes the assumed Archie's parameters and fluid properties for the gas-bearing carbonate field.

Figure 5.5 shows the field measurements together with core measurements, compared to results obtained with the interactive interpretation. The neutron-density overlay in panel b of Figure 5.5 emphasizes the matrix crossover because the reservoir is primarily of dolomite lithology. The gas flag in panel $\mathrm{j}$, proportional to hydrocarbon pore volume, is most pronounced across XY50 - XY75 ft despite the suppressed gas crossover in panel b. Across the interval in Figure 5.5, the gas flag provides a qualitative and unequivocal indication of hydrocarbon saturation despite mud-filtrate invasion and matrix crossover.

The calculated $\rho_{h c}$ in panel h of Figure 5.5, with an average value of $0.176-\mathrm{g} / \mathrm{cm}^{3}$, confirms that the reservoir is largely saturated with gas. Conclusively, I implement combined matrix and fluid volumetric analysis with the SNUPAR-based solver, where methane gas of $0.176 \mathrm{~g} / \mathrm{cm}^{3}$ is assumed as a component of the fluids, thus eliminating the water-filled assumption in the independent matrix analysis. Panel i shows cumulative plots of the volumetric fractions of shale, quartz, calcite, dolomite, water, and gas, obtained from the SNUPAR-based solver. The estimated $\rho_{m}$ and $\phi_{t}$ (panels e and f, respectively), agree well with core measurements. On the other hand, log-derived $S_{w}$ (panel g) within interval XX25 - XY00 ft is considerably lower than core measurements. 
This behavior can be attributed to variations in Archie's parameters for differing rock types along the well. Furthermore, $S_{w}$ in core samples could increase due to quick spurt loss in low porosity, low pressure reservoirs (Xu et al., 2012).

\subsubsection{Field Example II, Oil-Bearing Shale Example}

In this example, nuclear and array induction resistivity logs are acquired in a well drilled with oil-base mud across an oil-bearing shale formation from the Eagle Ford shale play. Table 5.4 describes the assumed fluid properties and Archie's parameters for the oil-bearing shale reservoir. Figure 5.6 shows field measurements, core measurements, and interpreted petrophysical properties for the oil-bearing shale example. Here, the SNUPAR-based matrix analysis assumes kerogen $\left(\mathrm{C}_{100} \mathrm{H}_{100} \mathrm{O}_{8}\right.$ of density $\left.1.4 \mathrm{~g} / \mathrm{cm}^{3}\right)$, calcite, kaolinite, and illite as components of the matrix. Panel e of Figure 5.6 compares $\rho_{m}$ from the interactive analysis to core measurements and elemental capture spectroscopy (ECS) lithology analysis. The ECS-derived $\rho_{m}$ (dashed blue curve) is significantly larger than core $\rho_{m}$ (blue circle points). This result is attributed to the exclusion of low-density kerogen matrix from the ECS analysis. Matrix density, $\rho_{m}$, from SNUPAR-based matrix analysis (red curve) agrees well with core measurements. The resulting fluid crossover, in panel b of Figure 5.6, after matrix-hydrogen and shalehydroxyl corrections, is due to combined effects of $\rho_{m}$ (less than $2.71 \mathrm{~g} / \mathrm{cm}^{3}$ of limestone), fluid density, and fluid hydrogen index. It is found that the interactive

analysis yields a relatively constant $\rho_{h c}$ of $0.747 \mathrm{~g} / \mathrm{cm}^{3}$ for the interval in panel $\mathrm{h}$. Furthermore, estimated $\phi_{t}$ and $S_{w}$ from the interactive analysis (panels $\mathrm{f}$ and $\mathrm{g}$, respectively), agree well with core measurements. 


\subsection{FLUID ZONE IDENTIFICATION}

Conventional methods for fluid contact identification include interpretation of pressure gradients due to fluid density differences in the reservoir hydrostatic column. Additionally, impermeable sealing or geological barriers, often at residual saturations, prevent hydraulic communication between fluid zones such that a higher density fluid resides above a lower density fluid in the hydrocarbon column.

Occasionally, when pressure measurements are unavailable or expensive to acquire, well logs are used to infer fluid zones. In this section, the estimated $\rho_{h c} \log$ yielded by the interactive interpretation is used for a quick-look qualitative identification of hydrocarbon zones and fluid contacts along the reservoir column in two more field examples.

\subsubsection{Field Example III, Identification of Hydrocarbon Fluid Contacts in a North Sea Siliciclastic Reservoir}

This example consists of a siliciclastic reservoir located in the central North Sea, where rock formations consist of noncalcareous mudstones interbedded with shaly sand deposits (Heidari et al., 2012). Panels a-d in Figure 5.7 show nuclear and array induction resistivity measurements acquired in a vertical well drilled with oil-base mud, while Table 5.5 summarizes the assumed properties and Archie's parameters for the siliciclastic reservoir. In addition, available pressure data in panel $\mathrm{f}$ describe three distinct and approximately constant pressure gradients. Water saturation, $S_{w}$, shown in panel e, estimated using the dual-water resistivity model, indicates that the hydrocarbon column exhibits a complete capillary transition with an aquifer below X750 ft. Pressure gradients identify three fluid zones, i.e., gas of $0.263 \mathrm{~g} / \mathrm{cm}^{3}$ density, oil of $0.647 \mathrm{~g} / \mathrm{cm}^{3}$ density, and an aquifer at residual hydrocarbon saturation with connate water of $1.005 \mathrm{~g} / \mathrm{cm}^{3}$ density, distinguished by red, green, and black intervals, respectively. Panel g shows the fluid 
densities, fluid zones, and fluid contacts, where estimated fluid densities, $\rho_{f, p}$, from pressure gradients are juxtaposed with $\rho_{f}$ estimated with the interactive interpretation.

Qualitatively, $\rho_{f}$ (panel g) and $\phi_{t}$ (panel h) from the interactive interpretation agree well with pressure and core measurements, respectively, except across the interval between X330 - X530 ft. This interval consists of highly inter-bedded sand-shale sequences; evident from GR $\log$ in panel a, whereby log-derived $\phi_{t}$ and $\rho_{f}$ are significantly influenced by shoulder-bed effects, and depth-by-depth analysis is inadequate. Note that the estimated $\rho_{f}$ from the interactive interpretation agrees well with $\rho_{f, p}$ across the thick bed layers in the gas zone. Nonetheless, assuming no reservoir compartmentalization and good hydraulic communication, the gas-oil contact (GOC) is located at X528 ft, while the oil-water contact (OWC) is located at X737 ft where the water zone is at residual hydrocarbon saturation.

\subsubsection{Field Example IV, Identification of Reservoir Compartments in the Deepwater Gulf of Mexico}

In this example, the reservoir consists of channel levees located in the deepwater Gulf of Mexico, where formations consist of unconsolidated shaly sand intervals and are primarily saturated with oil. Table 5.6 summarizes the assumed properties used in the interactive interpretation with a dual-water resistivity model. In Figure 5.8, the panels describe well logs and interpretation results across a hydrocarbon-saturated interval in the Gulf of Mexico reservoir. Panel e shows that average total porosities in the clean and shaly sand layers are 0.2721 and 0.1724 , respectively. In panels c and g, I observe a gassaturated reservoir compartment between XX522 ft and XX528 ft, where gas density is $0.144 \mathrm{~g} / \mathrm{cm}^{3}$ and the neutron-density overlay exhibits significant gas crossover. The primary oil-saturated zone, between XX394 ft and XX474 ft, with an estimated oil density of $0.43 \mathrm{~g} / \mathrm{cm}^{3}$ is above the gas-saturated compartment at XX522 - XX528 $\mathrm{ft}$. The 
compartmentalization of the gas layer is possible because hydraulic communication is severed between the oil and gas zones by the interleaving impermeable non-net shale barriers. This example verifies the capability of the interactive interpretation workflow to distinguish between oil- and gas-saturated layers, irrespective of formation lithology. The workflow also provides an efficient qualitative method for identification of reservoir compartments separated by sealing barriers.

\subsection{CONCLUSIONS}

The interactive interpretation workflow re-scales the neutron-density overlay with corrected neutron and density apparent porosities in a variable matrix scale, for independent characterization of fluid effects. It was found that the SNUPAR-based matrix analysis, assuming fresh-water-filled formations, renders accurate estimations of matrix density even across hydrocarbon-saturated intervals. Such a result is due to the fact that formation fluids have negligible or no effect on PEF and GR logs. One limitation of the SNUPAR-based matrix analysis is that a priori qualitative knowledge of matrix components, i.e., lithology, clay mineral, etc., is essential for accurate estimation of matrix density. This is achieved by preliminary lithology or matrix identification crossplots, e.g. PEF- $\rho_{b}$, thorium-potassium, and PEF-potassium cross-plots (Schlumberger, 2009). Furthermore, the workflow assumes minimal shoulder-bed effects such that depthby-depth analysis is adequate for SNUPAR calculations. The uncertainty in estimated $\rho_{h c}$ increases in thinly-bedded intervals with pronounced shoulder-bed effects.

The merits of the SNUPAR-based interactive interpretation workflow include the following:

(1) unequivocal identification of hydrocarbon-saturated zones,

(2) model-consistent formation porosity, and 
(3) hydrocarbon density for gas/oil zone identification.

It was shown that the workflow incorporates interactive matrix corrections such that Gaymard-Poupon's formulation for lithology-independent porosity and hydrocarbon identification can be implemented for any neutron-density matrix scale and lithology (clean or/and shaly), especially in wells with limited data.

Synthetic and field examples of application indicate that lithology-independent porosity and hydrocarbon density can be efficiently estimated from conventional nuclear and resistivity logs for reliable and quantitative detection and appraisal of hydrocarbonsaturated sweet spots and non-viable zones. Furthermore, identification of fluid types in the reservoir column provides a qualitative means for determining fluid contacts and reservoir compartments. 
Table 5.1: Layer properties assumed in the Synthetic Example.

\begin{tabular}{|c|c|c|c|}
\hline Layer & Matrix & Saturation fluid properties & $\begin{array}{l}\text { Interpretation } \\
\text { comments }\end{array}$ \\
\hline $\mathbf{I}$ & $\begin{array}{l}\text { Shale: } 80 \% \text { Illite, } \\
20 \% \text { Orthoclase, } \\
\rho_{s h}=2.738 \mathrm{~g} / \mathrm{cm}^{3}\end{array}$ & $\begin{array}{l}\phi_{t}=0.10 \\
S_{w}=1, S_{h c}=0\end{array}$ & $\begin{array}{l}\text { Shale and matrix } \\
\text { effects }\end{array}$ \\
\hline II & Limestone & $\begin{array}{l}\phi_{t}=0.28, S_{w}=0.05, S_{h c}=0.95 \\
\left(\text { Methane }, \mathrm{CH}_{4} 0.182 \mathrm{~g} / \mathrm{cm}^{3} \text { ) }\right.\end{array}$ & Gas effect \\
\hline III & Limestone & $\begin{array}{l}\phi_{t}=0.28, S_{w}=0.05, S_{h c}=0.95 \\
\text { (Liquid hydrocarbon, } \\
\mathrm{C}_{16} \mathrm{H}_{34} 0.757 \mathrm{~g} / \mathrm{cm}^{3} \text { ) }\end{array}$ & Hydrocarbon effects \\
\hline IV & $\begin{array}{l}\text { Shale: } 80 \% \text { Illite, } \\
20 \% \text { Orthoclase }\end{array}$ & $\phi_{t}=0.05, S_{w}=1, S_{h c}=0$ & $\begin{array}{l}\text { Shale and matrix } \\
\text { effects }\end{array}$ \\
\hline $\mathbf{V}$ & $\begin{array}{l}\text { Shale: } 80 \% \text { Illite, } \\
20 \% \text { Orthoclase }\end{array}$ & $\begin{array}{l}\phi_{t}=0.10, S_{w}=0.20, S_{h c}=0.80 \\
\left(\text { Methane, } \mathrm{CH}_{4} 0.182 \mathrm{~g} / \mathrm{cm}^{3}\right)\end{array}$ & Shale and gas effects \\
\hline VI & Dolomite & $\begin{array}{l}\phi_{t}=0.28, S_{w}=0.05, S_{h c}=0.95 \\
\text { (Liquid hydrocarbon, } \\
\mathrm{C}_{16} \mathrm{H}_{34} 0.757 \mathrm{~g} / \mathrm{cm}^{3} \text { ) }\end{array}$ & $\begin{array}{l}\text { Matrix and } \\
\text { hydrocarbon effects }\end{array}$ \\
\hline VII & Dolomite & $\begin{array}{l}\phi_{t}=0.10 \\
S_{w}=1, S_{h c}=0\end{array}$ & Matrix effects \\
\hline VIII & Limestone & $\phi_{t}=0$ & Limestone reference \\
\hline
\end{tabular}

Table 5.2: Summary of assumed Archie's parameters and fluid properties for the Synthetic Example.

\begin{tabular}{|l|c|c|}
\hline Variable & Value & Units \\
\hline Connate water resistivity, $R_{w} @ 200^{\circ} \mathrm{F}$ & 0.0203 & $\Omega-\mathrm{m}$ \\
\hline Connate water density, $\rho_{c w}$ & 1.11 & $\mathrm{~g} / \mathrm{cm}^{3}$ \\
\hline Connate water hydrogen index, $H I_{c w}$ & 0.936 & - \\
\hline Connate water salt concentration & 160,000 & $\mathrm{ppm} \mathrm{NaCl}$ \\
\hline Archie's factor, $a$ & 1 & - \\
\hline Archie's porosity exponent, $m$ & 1.95 & - \\
\hline Archie's saturation exponent, $n$ & 1.75 & - \\
\hline
\end{tabular}


Table 5.3: Summary of assumed fluid properties and Archie's parameters for Field Example I, gas-bearing carbonate.

\begin{tabular}{|l|c|c|}
\hline Variable & Value & Units \\
\hline Connate water resistivity, $R_{w} @ 96^{\circ} \mathrm{F}$ & 0.04 & $\Omega-\mathrm{m}$ \\
\hline Connate water density, $\rho_{c w}$ & 1.12 & $\mathrm{~g} / \mathrm{cm}^{3}$ \\
\hline Connate water hydrogen index, $H I_{c w}$ & 0.932 & - \\
\hline Connate water salt concentration & 170,000 & $\mathrm{ppm} \mathrm{NaCl}$ \\
\hline Mud-filtrate water resistivity, $R_{m f} @ 96^{\circ} \mathrm{F}$ & 0.84 & $\Omega-\mathrm{m}$ \\
\hline Mud-filtrate water density, $\rho_{m f}$ & 1 & $\mathrm{~g} / \mathrm{cm}^{3}$ \\
\hline Mud-filtrate hydrogen index, $H I_{m f}$ & 1 & - \\
\hline Mud-filtrate water salt concentration & 5147 & $\mathrm{ppm} \mathrm{NaCl}$ \\
\hline Archie's factor, $a$ & 1 & - \\
\hline Archie's porosity exponent, $m$ & 1.96 & - \\
\hline Archie's saturation exponent, $n$ & 1.83 & - \\
\hline
\end{tabular}

Table 5.4: Summary of assumed fluid properties and Archie's parameters for Field Example II, oil-bearing shale.

\begin{tabular}{|l|c|c|}
\hline Variable & Value & Units \\
\hline Connate water resistivity, $R_{w} @ 215^{\circ} \mathrm{F}$ & 0.019 & $\Omega-\mathrm{m}$ \\
\hline Connate water density, $\rho_{c w}$ & 1.077 & $\mathrm{~g} / \mathrm{cm}^{3}$ \\
\hline Connate water hydrogen index, $H I_{c w}$ & 0.901 & - \\
\hline Connate water salt concentration & 165,000 & $\mathrm{ppm} \mathrm{NaCl}$ \\
\hline Archie's factor, $a$ & 1 & - \\
\hline Archie's porosity exponent, $m$ & 2.1 & - \\
\hline Archie's saturation exponent, $n$ & 2 & - \\
\hline
\end{tabular}


Table 5.5: Summary of assumed properties and Archie's parameters for Field Example III, North Sea siliciclastic reservoir.

\begin{tabular}{|l|c|c|}
\hline Variable & Value & Units \\
\hline Connate water resistivity, $R_{w} @ 254^{\circ} \mathrm{F}$ & 0.025 & $\Omega-\mathrm{m}$ \\
\hline Connate water density, $\rho_{c w}$ & 1.005 & $\mathrm{~g} / \mathrm{cm}^{3}$ \\
\hline Connate water hydrogen index, $H I_{c w}$ & 0.997 & - \\
\hline Connate water salt concentration & 77,600 & $\mathrm{ppm} \mathrm{NaCl}$ \\
\hline Archie's factor, $a$ & 1 & - \\
\hline Archie's porosity exponent, $m$ & 1.89 & - \\
\hline Archie's saturation exponent, $n$ & 1.92 & - \\
\hline Shale porosity, $\phi_{s h}$ & 0.10 & $\mathrm{v} / \mathrm{v}$ \\
\hline Shale resistivity, $R_{s h}$ & 1.50 & $\Omega-\mathrm{m}$ \\
\hline
\end{tabular}

Table 5.6: Summary of assumed properties and Archie's parameters for Field Example IV, deepwater Gulf of Mexico reservoir.

\begin{tabular}{|l|c|c|}
\hline Variable & Value & Units \\
\hline Connate water resistivity, $R_{w} @ 150^{\circ} \mathrm{F}$ & 0.030 & $\Omega-\mathrm{m}$ \\
\hline Connate water density, $\rho_{c w}$ & 1.098 & $\mathrm{~g} / \mathrm{cm}^{3}$ \\
\hline Connate water hydrogen index, $H I_{c w}$ & 0.9441 & - \\
\hline Connate water salt concentration & 140,000 & $\mathrm{ppm} \mathrm{NaCl}$ \\
\hline Archie's factor, $a$ & 1 & - \\
\hline Archie's porosity exponent, $m$ & 1.92 & - \\
\hline Archie's saturation exponent, $n$ & 2.00 & - \\
\hline Shale porosity, $\phi_{s h}$ & 0.15 & $\mathrm{v} / \mathrm{v}$ \\
\hline Shale resistivity, $R_{s h}$ & 1.0 & $\Omega-\mathrm{m}$ \\
\hline
\end{tabular}




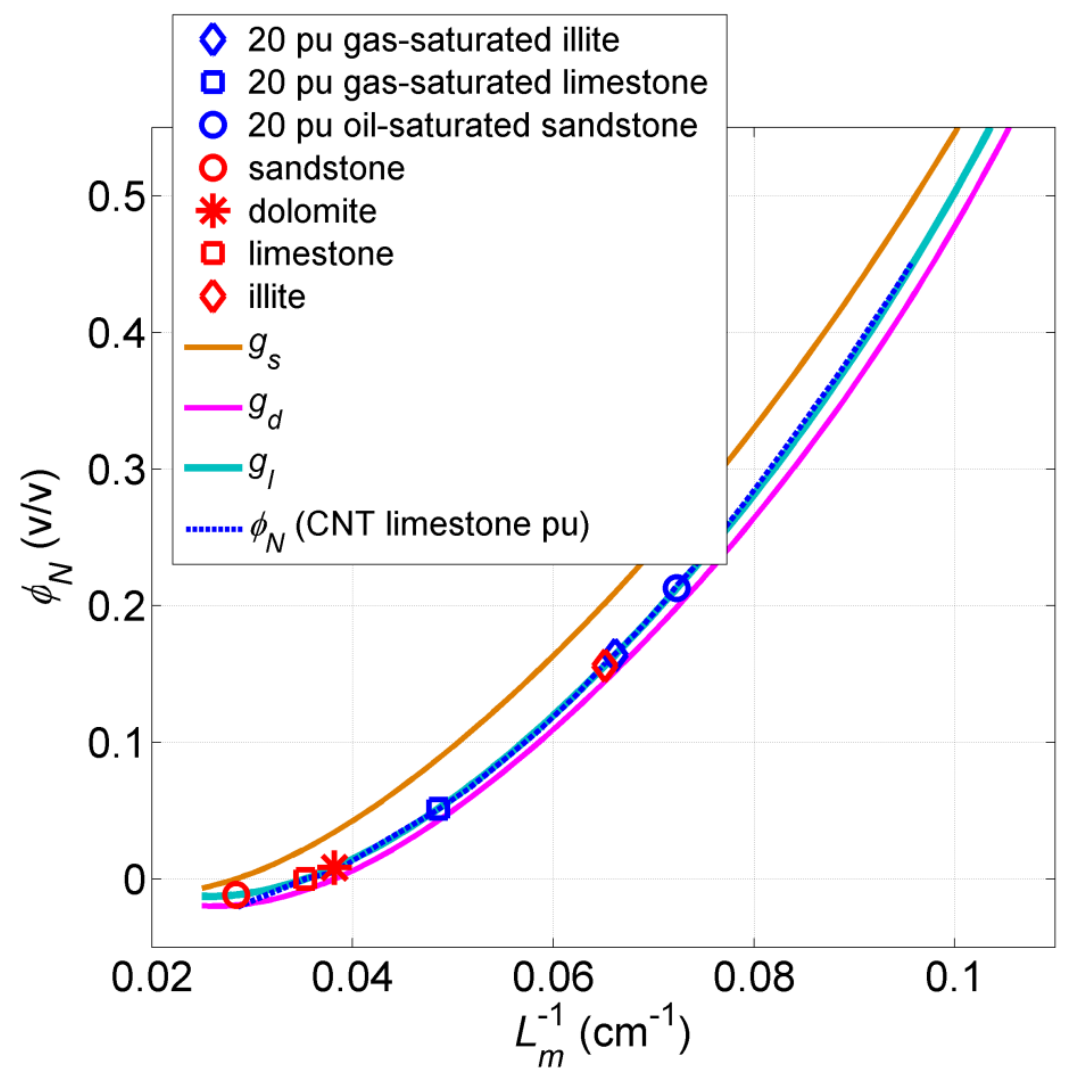

Figure 5.1: SNUPAR-calculated water-filled neutron porosity calibration functions $g_{s}$, $g_{l}$, and $g_{d}$ for sandstone, limestone, and dolomite units, respectively. The figure also shows neutron porosity responses across relevant formations. 


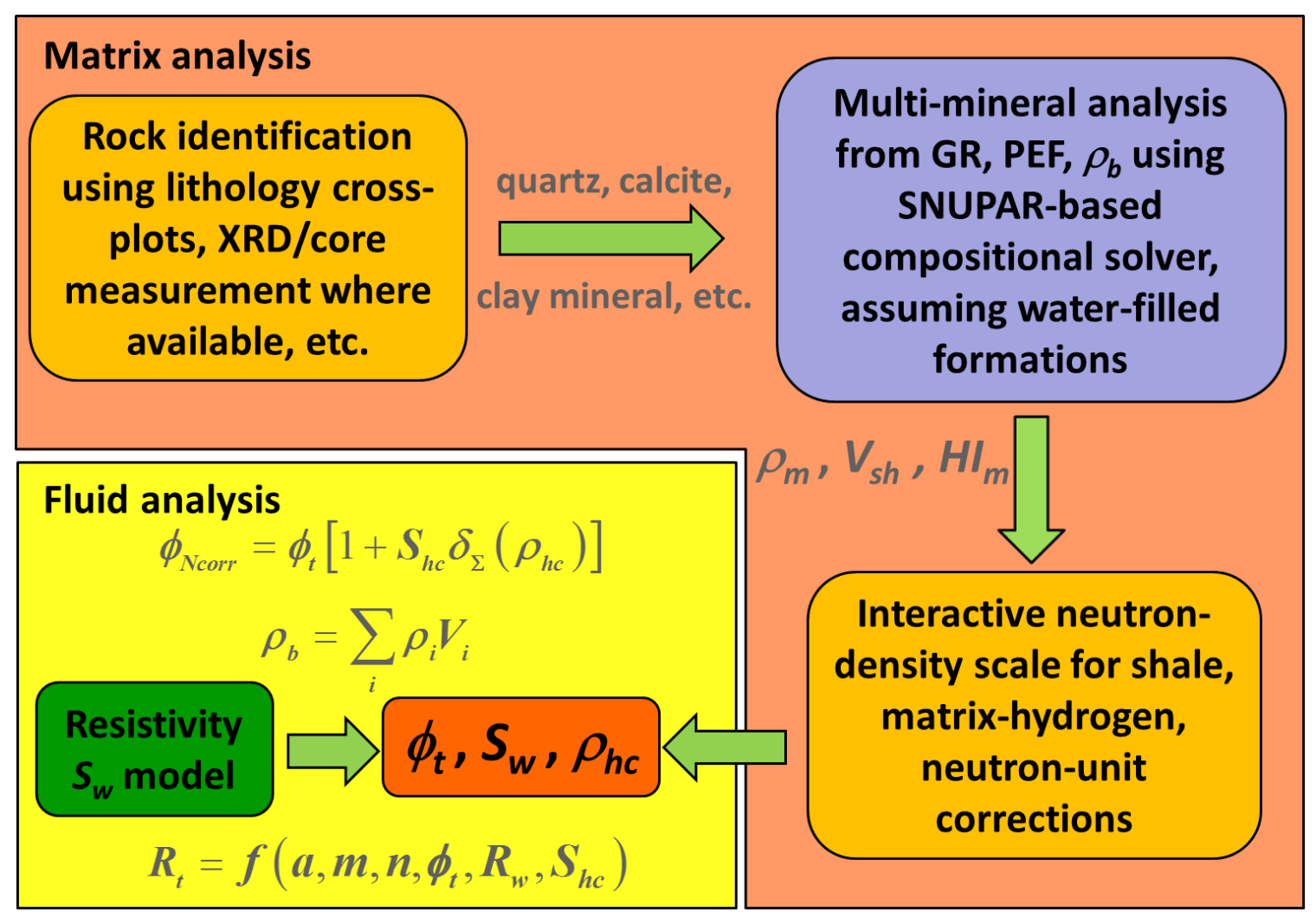

Figure 5.2: Interactive interpretation workflow for interpretation of neutron and density apparent porosities. 


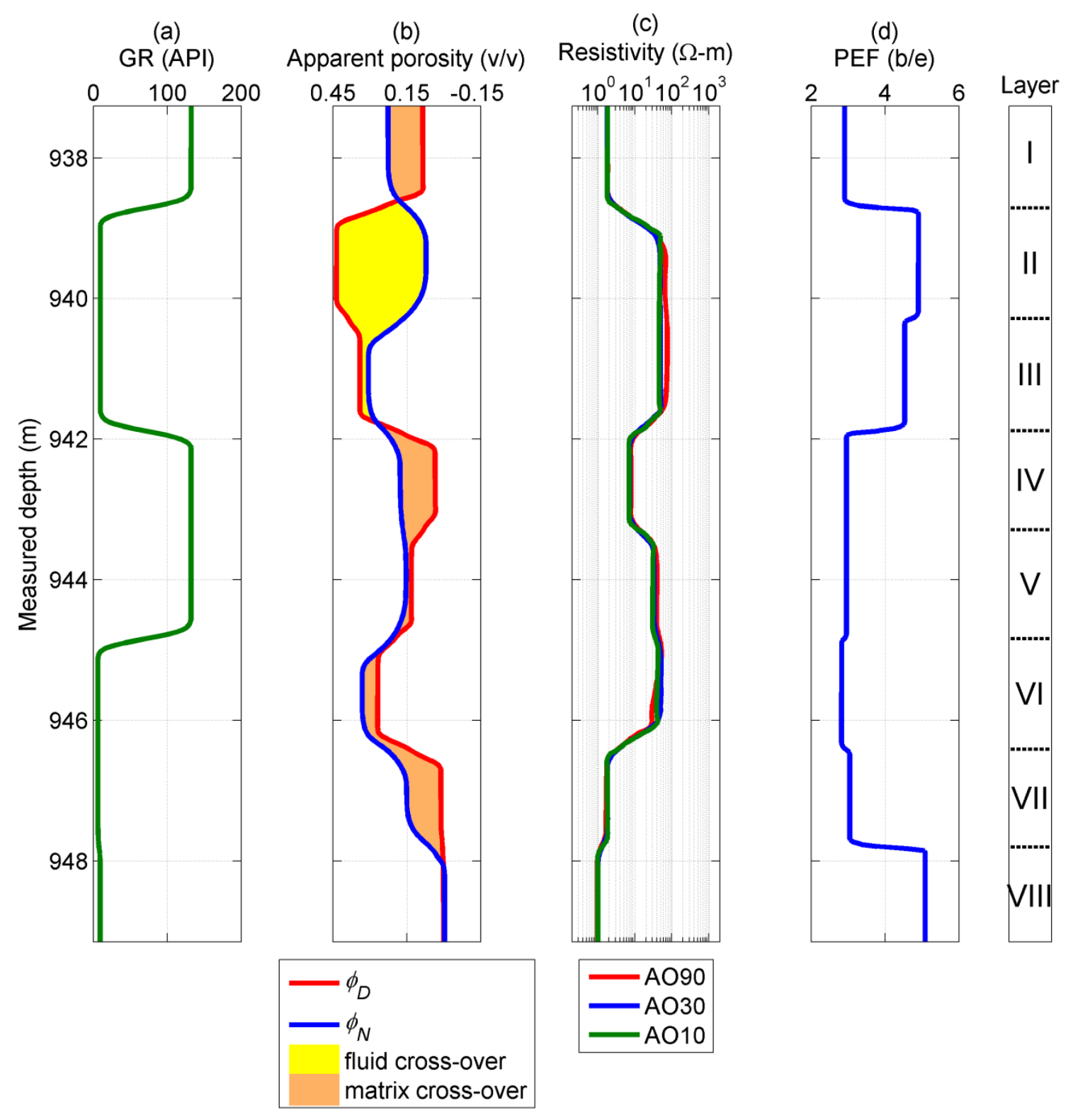

Figure 5.3: Simulated well logs across the synthetic multi-layer model. (a) Gamma ray (GR) $\log$, (b) neutron and density apparent porosities on a limestone scale, (c) array induction apparent resistivity logs, and (d) PEF log. Refer to Table 5.1 for a description of assumed layer properties. 


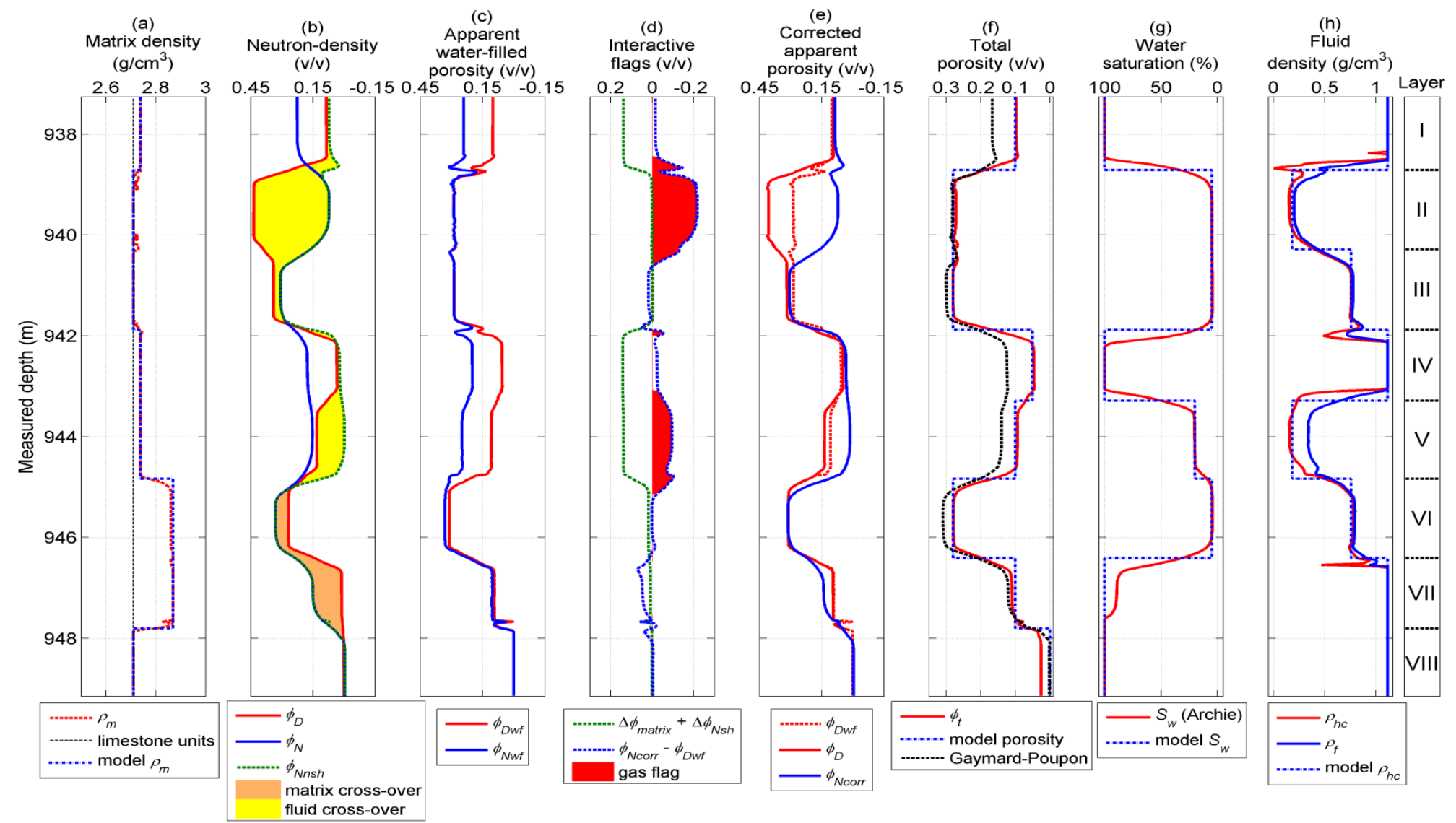

Figure 5.4: Interpretation results for the Synthetic Example using the interactive interpretation workflow. (a) Interpreted matrix density from SNUPAR-based matrix solver, (b) neutron-density overlay showing shale-corrected neutron log, matrix and fluid crossover characteristics, (c) neutron and density apparent water-filled logs from SNUPAR-based matrix solver, (d) interactive flag indicators showing matrix effect and gas flag, (e) corrected neutron-density overlay, (f) estimated total porosity, (g) estimated water saturation, and (h) estimated hydrocarbon and fluid densities. Refer to Table 5.1 for a description of layer properties. 

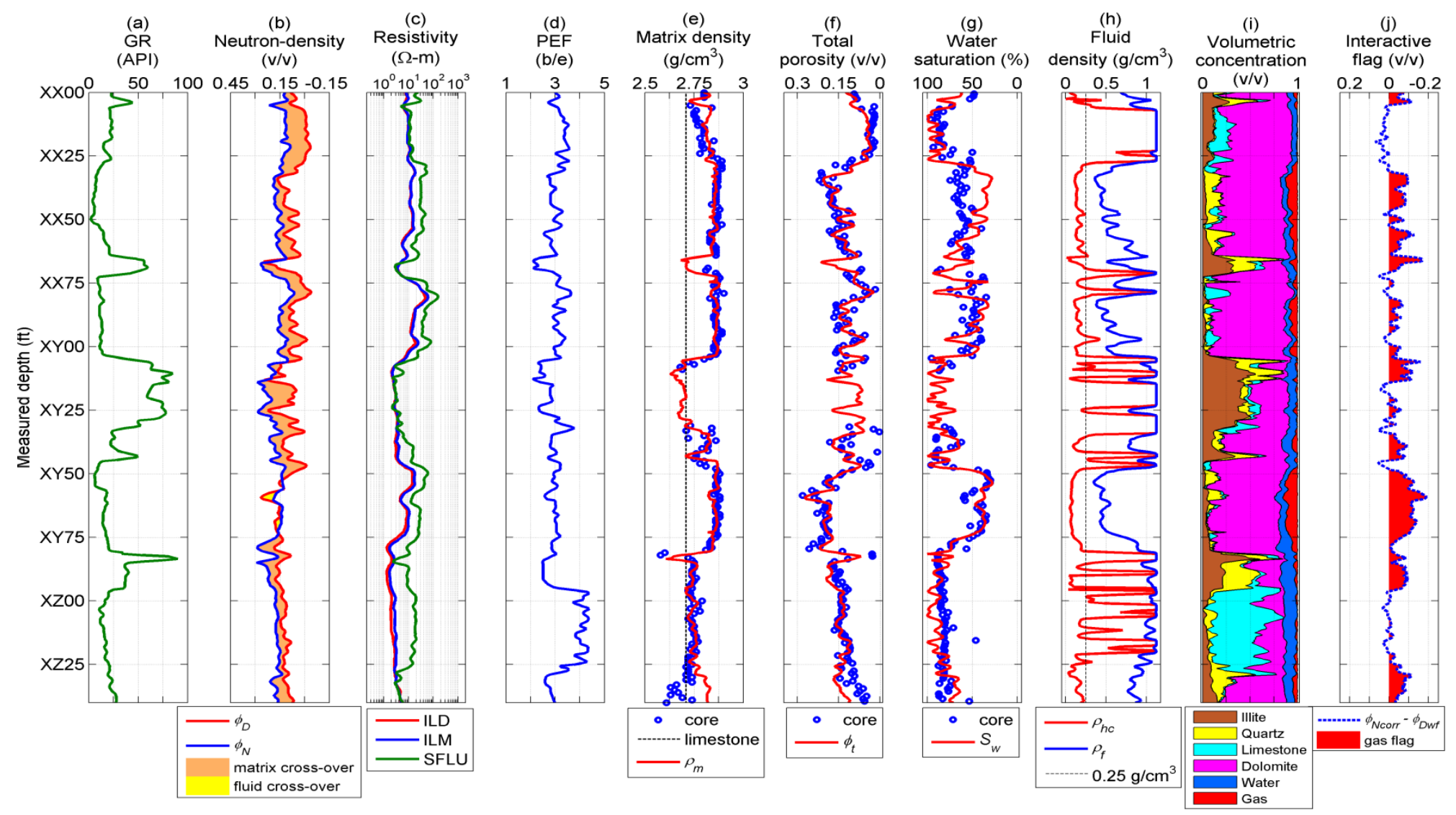

Figure 5.5: Interpretation results for Field Example I, gas-bearing carbonate reservoir, using the interactive interpretation workflow. (a) Gamma ray log, (b) neutron and density porosities on limestone scale, (c) dual-induction resistivity logs, and (d) photoelectric factor log. (e) Matrix density, (f) total porosity, and (g) water saturation from core measurements and interactive analysis. (h) Calculated fluid densities showing a gas cut-off of $0.25 \mathrm{~g} / \mathrm{cm}^{3}$. (i) Volumetric concentrations of rock and fluid components from SNUPAR-based solver. (j) Gas flag from interactive analysis workflow. 


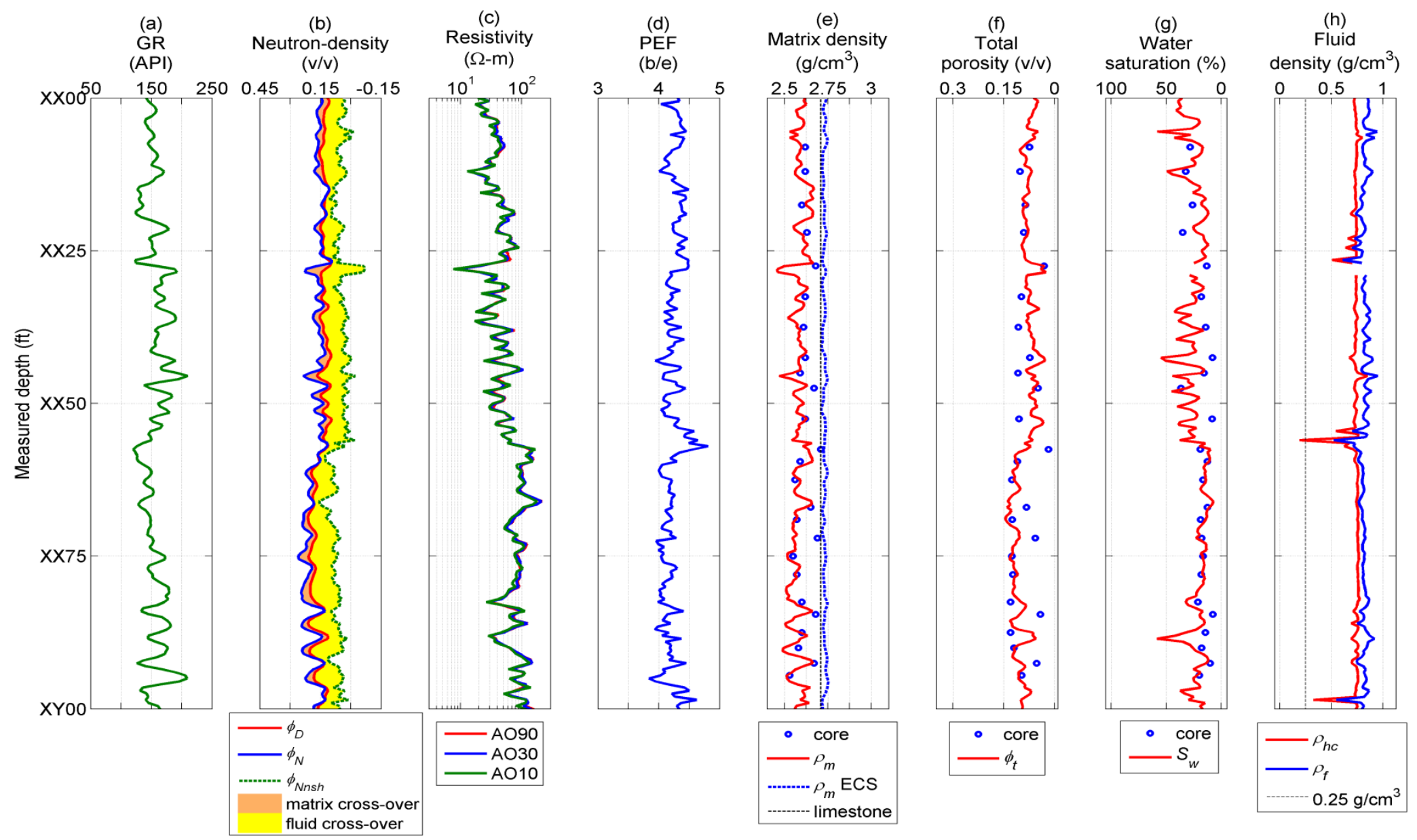

Figure 5.6: Interpretation results for Field Example II, oil-bearing shale reservoir, using the interactive analysis workflow. (a) Gamma ray log, (b) neutron and density porosities on limestone scale, (c) array induction resistivity logs, and (d) photoelectric factor log. (e) Matrix density, (f) total porosity, and (g) water saturation from core measurements and interactive analysis. (h) Calculated fluid densities showing a gas cut-off of $0.25 \mathrm{~g} / \mathrm{cm}^{3}$. 


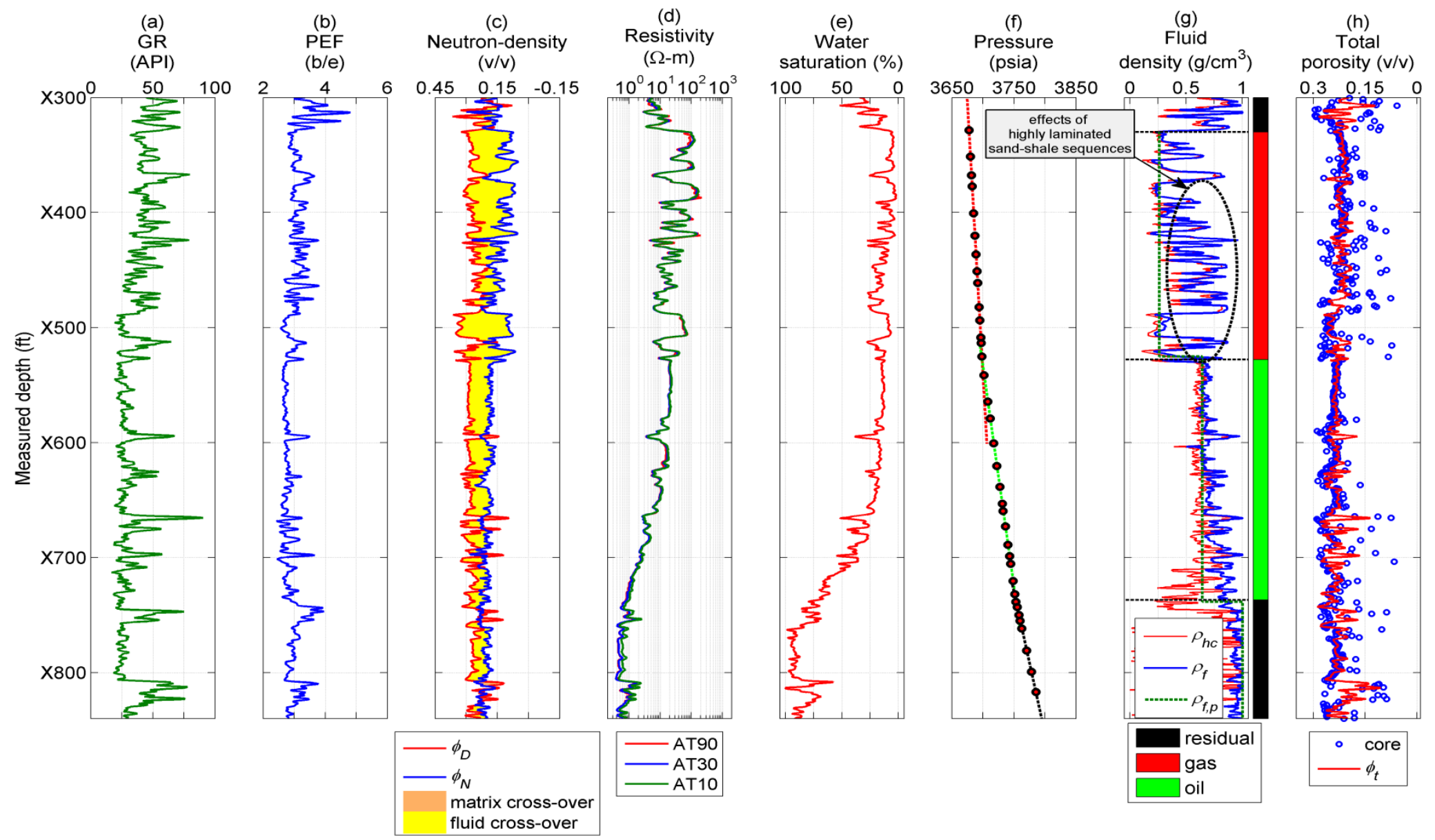

Figure 5.7: Fluid zone interpretation results for Field Example III, North Sea siliciclastic reservoir. (a) Gamma ray log, (b) photoelectric factor $\log$, (c) neutron and density porosities on limestone scale, and (d) array induction resistivity logs. (e) Estimated water saturation, (f) pressure measurements, and (g) fluid densities from interactive analysis and pressure gradients. (h) Total porosity from core measurements and interactive analysis. 

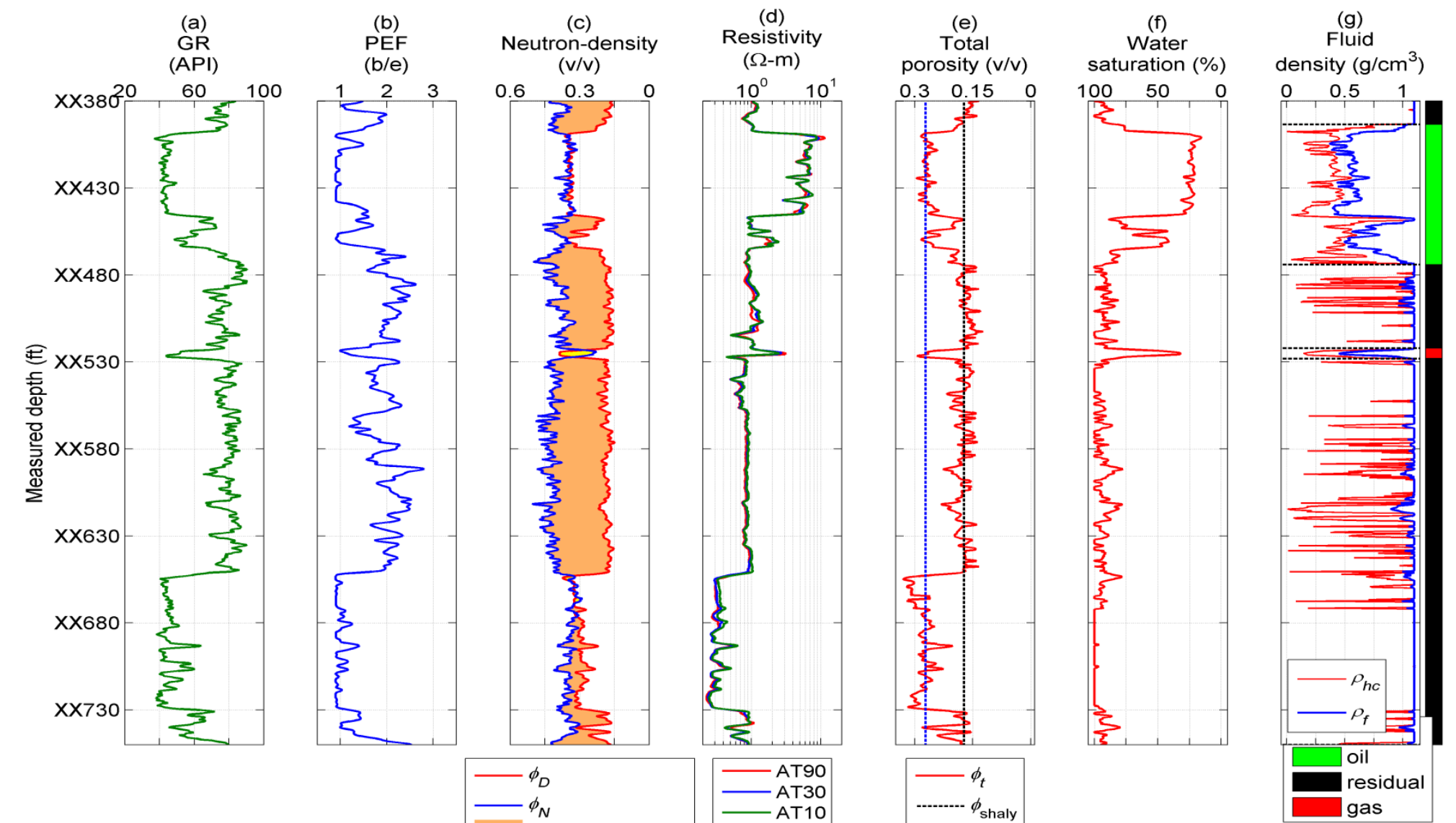

Figure 5.8: Fluid zone interpretation results for Field Example IV, deepwater Gulf of Mexico reservoir. (a) Gamma ray log, (b) photoelectric factor $\log$, (c) neutron and density porosities on sandstone scale, and (d) array induction resistivity logs. (e) Total porosity, (f) water saturation, and (g) fluid density logs estimated using the interactive analysis. 


\section{Chapter 6: Summary, Conclusions, and Recommendations}

This final chapter summarizes the developments and technical contributions in the dissertation, draws general conclusions from the results, and provides recommendations for future research. Figure 6.1 summarizes the three parts and main objectives of the dissertation using a descriptive chart.

\subsection{SUMMARY}

The main objective of the dissertation was to develop an inversion-based algorithm for petrophysical interpretation of logging-while-drilling (LWD) nuclear and resistivity measurements acquired in high-angle and horizontal (HA/HZ) wells. The inversion algorithm implements forward models that invoke multiple borehole measurement physics and accounts for petrophysical and geometrical effects in the interpretation while drilling.

Accurate and efficient petrophysical modeling techniques that simulate and invert neutron porosity logs in the presence of borehole environmental effects and across complex formation geometries have been elusive. This is the main motivation of the first part of the dissertation; where I introduced two new methods for numerical simulation of neutron porosity logs: (1) neutron-density $F_{p}$ petrophysical parameterization, and (2) transport-diffusion modeling. The first method describes a new formation parameter, $F_{p}$, obtained by combining neutron characteristic lengths and bulk density. The $F_{p}$ fits detector responses, irrespective of connate water salinity and saturating gas density, for reliable prediction of borehole environmental effects in a fast-forward model. In the second method, I developed a new neutron transport-diffusion approximation for estimating spatial neutron flux perturbations. Both methods were validated with full Monte Carlo (MC) calculations. It was found that the transport-diffusion approximation 
was more robust and accurate than the $F_{p}$ petrophysical parameter because it honored the physics of neutron transport. Moreover, the transport-diffusion approximation is two times faster than the linear iterative refinement (LIR) and it serves as a semi-analytical form for calculating entries of the Jacobian matrix for inversion applications.

In the second part of the dissertation, I introduced an efficient inversion-based interpretation workflow for integrating LWD multi-sector nuclear and multi-array resistivity measurements acquired in highly-deviated and horizontal wells. The workflow is summarized in three steps: (1) consolidated geometrical interpretation of density, photoelectric factor (PEF), and natural gamma ray (GR) measurements to yield a $2 \mathrm{D}$ layer-based formation model, (2) separate nonlinear inversion within respective nuclear and resistivity domains where 3D multi-physics Jacobian operator matrices and forward models describe petrophysical and geometrical effects, and (3) layer-by-layer compositional interpretation with a physics (SNUPAR)-based multi-mineral solver for volumetric estimation of rock and fluid constituents. I applied the workflow to challenging synthetic and field examples to verify its reliability and efficiency. Furthermore, I calculated confidence intervals to measure stability of inversion results, sensitivity of the layer-based model, and accuracy of rock/fluid estimations. The inversion-based interpretation workflow corrects LWD measurements for geometrical effects ensuing from well trajectory, shoulder-bed properties, and differing volumes of investigation. Results indicated that conventional interpretation methods (applied in vertical wells) that do not correct such geometrical effects can give rise to inaccurate estimation of hydrocarbon pore volume (HPV) when applied in HA/HZ wells. In the dissertation, observed errors in HPV were as high as $15 \%$ and $10 \%$ when performing conventional interpretation methods in high-angle and horizontal wells, respectively. 
The final part of the dissertation introduced a method that implements the SNUPAR-based multi-mineral/compositional solver (discussed in the third step of the second part of the dissertation) to calculate a variable and interactive neutron-density matrix scale. The SNUPAR-based compositional solver was used to quantify matrix effects for depth-by-depth correction of neutron and density porosity logs. Interpreted matrix effects included shale-hydroxyl, matrix-hydrogen, and lithology type. Subsequent to matrix corrections, the method inferred saturating fluid effects from neutron, density, and resistivity logs for estimation of hydrocarbon densities and pore volumes. To assess the reliability of this technique, I applied it to field examples of carbonate, shale-gas, and siliciclastic reservoirs. Results were also validated with laboratory core measurements whenever available.

\subsection{CONCLUSIONS}

This section lists the general and specific conclusions stemming from the research documented in the chapters describing the three parts of this dissertation.

\subsubsection{General Conclusions about the Significance to Formation Evaluation and Best Practices for Petrophysical Modeling and Inversion of LWD Measurements}

i. The main contribution and significance of this dissertation is the development of modeling-based interpretation techniques for improved petrophysical quantification and formation evaluation in $\mathrm{HA} / \mathrm{HZ}$ wells.

ii. The techniques documented in this dissertation endeavor to maintain computational efficiency for potential practical applications in real-time, whiledrilling, and modeling-based petrophysical analysis. Such objectives include geosteering applications for better well placement and targeting of pay zones. 
iii. Complex variations (e.g., resistivity polarization horns and density image "bull'seye" feature) in LWD data due to bed geometrical properties could be overwhelming to a novice petrophysicist. Common practice involves averaging azimuthal measurements for petrophysical evaluation. Business decisions about drilling, perforation, or side-tracking commonly made in $\mathrm{HA} / \mathrm{HZ}$ wells based on such conventional can analysis introduce larger risks, and should be considered bad practice.

iv. Special considerations for well-log interpretation include proper depth matching of well logs before performing combined inversion. Occasionally, field measurements exhibit depth shifts due to improper corrections for varying radial depths of investigation of the multiple measurements. Such depth shifts can be accentuated in $\mathrm{HA} / \mathrm{HZ}$ wells. The inversion-based workflow corrects for such varying radial depths of investigation within each measurement domain before performing combined multi-mineral analysis.

v. Generally, nuclear and resistivity measurements exhibit nonlinear response behavior with respect to variations of formation petrophysical properties, especially across complex formation lithologies. It follows that nonlinear minimization is most appropriate for well-log and multi-mineral inversion.

vi. Gradient-based nonlinear iterative inversion methods (Gauss-Newton, LevenbergMarquardt, and Occam) assume approximate quasi-linear gradients per iteration. Therefore, quasi-linear transforms of tool responses or/and formation properties can improve convergence of the nonlinear inversion. For example, in the neutron porosity domain, the inverse of migration length is selected as the inverted parameter because it is directly proportionally to neutron macroscopic crosssection. Additionally, in the resistivity domain, apparent conductivity, i.e. inverse 159 
of apparent resistivity, is implemented for data inversion. Furthermore, general tests with inversion of resistivity or its logarithm indicated an increase in the quadratic error cost function for subsequent iterations, i.e. worse stability than for the case of inversion of apparent conductivity. An oversimplification that applies linear inversion could be more computationally efficient but unacceptable in terms of accuracy and uniqueness.

vii. One factor contributing to the efficiency of the inversion-based interpretation method is the stability of inversion results. Stability is investigated with the speed of convergence to a minimum error, change in the quadratic cost function for subsequent iterations, and condition number of the forward or Jacobian operator matrix. The Tikhonov regularization method (Aster et al., 2005) is the common formulation for stabilizing inversion calculations. In the course of the research documented in this dissertation, I found that the generalized cross-validation (GCV) criterion was appropriate for selecting the regularization parameter used to stabilize nonlinear inversion of well logs. The GCV criterion, often referred to as "leave-one-out lemma," has desirable statistical properties including minimization of the predictive error in the case where data are missing.

viii. Because the measurement physics is explicitly considered in separate forward models, I categorized LWD measurements in the dissertation into separate domains to ensure stability of the separate inversion. Nonlinear joint well-log inversion methods that combine all the measurements' forward operator functions in a single operator matrix tend to be ill-conditioned and unstable. Occasionally, these operator matrices are obtained by brute-force numerical approximations of the partial derivatives, thereby becoming more computationally inefficient than separate inversion. 
ix. Field propagation electromagnetic measurements are most commonly delivered as processed apparent resistivity logs. Raw conductivity measurements are seldom available to well-log analysts. Additionally, the ARC (Array Resistivity Compensation) forward model used for inversion of apparent conductivity measurements in the dissertation was implemented as a black-box simulator whereby entries of the Jacobian matrix were calculated with finite-difference approximations from multiple forward simulations. This is because methods used to process raw conductivities and calculate multi-resolution phase and attenuation apparent resistivities are often proprietary.

x. Without increasing the length of confidence intervals of inverted bed conductivities, the dimensions of the Jacobian matrix in the resistivity domain can be varied and controlled by performing inversion with selective resistivity channels. For example, I found that choosing the shallow and deep (16- and 40-in. arrays, respectively) apparent resistivity channels for inverting vertical and horizontal conductivities improved the stability and convergence of the inversion when quantifying the effects of formation anisotropy (Appendix C). This is an interesting observation because the shallow- and deep-sensing curves complementarily enforce the smallest and largest volumes of investigation, respectively.

xi. Commercial software usually implements non-deterministic and stochastic methods for multi-mineral analysis from well logs. The SNUPAR-based multimineral solver implements deterministic multi-group functions that relate rock/fluid volumetric concentrations to nuclear cross-sections. This approach introduces deterministic physical relationships into multi-mineral analysis, in comparison to probability functions often invoked by commercial solvers. 
xii. Field measurements delivered by LWD logging/acquisition runs are often contaminated with noise due to tool vibration and adverse borehole conditions. The relative amount of noise in the data decreases the confidence in interpreted formation properties. For this reason, confidence intervals should always accompany interpreted petrophysical estimations and multi-mineral analysis.

xiii. In the dissertation, I described calculation of $95 \%$ confidence intervals for inverted petrophysical properties using the model covariance in equation B.1 of Appendix B. The length of confidence intervals is qualitatively and quantitatively proportional to the degrees of (a) non-uniqueness of inverted results, (b) instability in the inverse problem, and (c) relative energy of noise present in the data. For example, when layers are thinner than the resolution of the measurements, calculated confidence intervals across thin beds tend to increase in relation to the value of the inverted property. This behavior is especially indicative of non-uniqueness. Another factor that contributes to the relative size of confidence intervals is numerical uncertainty in the approximated entries of the Jacobian matrices.

xiv. The computed confidence interval assumes a normal distribution with its mean equal to the inverted result and its variance equal to covariance matrix of the model solution. This allows inversion to detect biases or inconsistencies (e.g., borehole environment effects and noise), in the data, that do not average out in a least-squares manner.

\subsubsection{Part One: Fast-Forward Modeling of Logging-While-Drilling Neutron Porosity Measurements in the Presence of Borehole and Environmental Effects}

i. The newly developed formation parameter, $F_{p}$, represents a neutron cross-section relevant to petrophysical properties used for describing FSF (flux sensitivity 
function) perturbations and for measuring neutron flux attenuation. Unlike the inverse of neutron migration length, it is suitable to describe detector responses irrespective of saturating gas densities.

ii. In conjunction with FSFs and LIR, the FSF- $F_{p}$ fast-forward model (FFM) was benchmarked with full MC calculations. It was found that simulation errors were maintained below 2 porosity units (pu). Additionally, I successfully validated the FFM with experimental neutron porosity data for a commercial LWD logging tool.

iii. In the dissertation, FSF- $F_{p}$ FFM required 0.47 seconds of CPU time per sample point on a Windows XP dual-core system while MCNP calculations required 17280 seconds on a Linux cluster. The model takes advantage of the efficiency in the FSF technique for fast simulation of raw neutron detector counts.

iv. Results obtained with the FSF- $F_{p}$ FFM confirm its applicability for modeling raw neutron detector responses with realistic stabilizer and borehole sizes. The method replicates measurement responses in the presence of borehole and environmental effects with errors below $2 \mathrm{pu}$. This property makes the simulation method a good candidate for inversion applications.

\subsubsection{Part One: Fast Modeling of Borehole Neutron Porosity Measurements with a New Spatial Transport-Diffusion Approximation}

i. I developed a fast neutron transport-diffusion approximation using one-group diffusion models as Green's function kernels for estimating 2D and 3D spatial diffusion flux-difference (DFD) functions. The DFD functions, along with transport-correction coefficients and homogeneous base-case FSFs, reproduce full MC-derived perturbed neutron FSFs with enhanced computational efficiency and accuracy. 
ii. Using the diffusion kernel functionalities, the DFD method focuses on modeling differences or perturbations in the adjoint/sensitivity flux, and not on the forward flux itself. It augments MC-derived FSFs that are pre-calculated for base-case homogeneous formations.

iii. The DFD formulation is efficient and accurate for calculating semi-analytical Jacobian operator matrices in the separate nonlinear inversion of LWD neutron porosity measurements.

iv. Transport-correction coefficients are found to be constant, per detector, per tool, for wireline and LWD tools, across various lithologies and fluid constituents. The coefficients adjust the neutron cross-sections to reproduce higher-order transport effects resulting from the transient part of neutron transport. Additionally, they modify the migration length to describe an apparent mean-free-path traveled by neutrons from their generation to their detection.

v. A limitation of the DFD technique is in modeling wireline measurements across tight and low porosity formations with high migration lengths (e.g. pyrite). In such formations, the assumed transport correction coefficients calculated in typical lithologies (sandstone, limestone, and dolomite) may be invalid.

vi. The DFD method, as documented in the dissertation, is more accurate than the LIR technique and the FSF- $F_{p}$ FFM. It maintains neutron porosity errors below 1 $\mathrm{pu}$, even for complex formation geometries. Additionally, it is two times faster than the LIR technique. The full MCNP calculations required 24 hours of CPU time per depth on a Linux cluster, while the LIR and DFD methods required 2.7 seconds and 1.4 seconds, respectively, on a Windows XP dual-core system for a 16-sector binning scheme. 
vii. The extended library for the LIR requires FSFs for multiple sector-binning orientations, boreholes sizes, and formation lithologies. By contrast, the DFD technique circumvents the need to pre-calculate such an extended library; it is therefore more efficient and easier to implement than the LIR for neutron porosity simulations for any given wireline or LWD tool. The implementation of the DFD technique could reduce computation time and storage disk space by a factor of 144.

viii. First-order neutron calculations could remain inaccurate when the degree of formation variability is significant, e.g. gas-saturated formations penetrated by highly-deviated wells with mud-filtrate invasion. The DFD technique yielded accurate calculations of neutron detector sensitivity functions and/or perturbations, for a wide range of borehole-formation conditions; it approximates higher-order terms in the Taylor's perturbation series with a lumped term obtained from the spatial DFD function. This is the reason why errors in forward simulation of neutron porosity with the DFD method are maintained below $1 \mathrm{pu}$.

ix. Both DFD and FSF- $F_{p}$ methods circumvent the need to pre-calculate FSFs for different azimuthal tool rotations, but the DFD technique is more efficient and accurate than the FSF- $F_{p}$ FFM for quantifying spatial neutron responses. Note that the FSF- $F_{p}$ still implements the LIR. It is for these reasons that the DFD method was chosen for inversion of neutron porosity measurements.

\subsubsection{Part Two: Inversion-Based Petrophysical Interpretation of Logging-While- Drilling Nuclear and Resistivity Measurements}

i. I successfully developed an algorithm for inversion-based interpretation of LWD multi-sector nuclear (density, neutron porosity, PEF, and natural GR) and multiarray apparent propagation resistivity measurements. The algorithm implements 
efficient property parameterization and stable nonlinear inversion calculations for separate measurement domains. Final results consist of layer-by-layer petrophysical and compositional formation properties, i.e. matrix density, total porosity, hydrocarbon saturation, and multi-mineral rock/fluid volumetric concentrations.

ii. I achieved an explicit 3D multi-physics description by invoking fast-forward models in each measurement domain for numerical construction of Jacobian matrices. The entries of the Jacobian matrices were obtained from nuclear FSFs and finite-difference approximations of partial derivatives calculated with rapid numerical simulations of well logs.

iii. I was prompted to infer the layer-based formation model from gamma (density, PEF, natural GR) measurements because of their relatively high vertical resolution. The layer-based model describes layers of locally distinct lithologies and petrophysical properties. Such a step in the interpretation workflow is crucial for estimation of reservoir pay because uncertainty in the constructed formation geometrical model could yield porosity errors as high as $20 \%$, especially across thinly bedded formations. The latter is an important limitation when implementing a layer-based model in the inversion-based workflow.

iv. I developed a SNUPAR-based multi-mineral/compositional solver that takes advantage of quantitative multi-group nuclear relationships. In comparison to commercial solvers, the SNUPAR-based solver deterministically relates rock and fluid properties to the physical parameters that are measured by nuclear logs. Furthermore, I successfully coupled the solver with water saturation and GR-shale volume models for estimation of grain density, formation porosity, and 
hydrocarbon volume. The solver was successfully implemented for the interactive matrix analysis workflow discussed in the final part of the dissertation.

v. Even though higher well deviation increases length exposure across a formation, the complex geometrical relationships between well trajectory and formation bed orientation is the primary reason why conventional interpretation techniques applied in vertical wells are not reliable in $\mathrm{HA} / \mathrm{HZ}$ wells. I verified that inversion explicitly corrects LWD measurements for such complex geometrical effects, whereby the interpreted true layer-by-layer properties are solely influenced by petrophysical effects.

vi. I confirmed that conventional interpretation methods developed for vertical wells, when applied in HA/HZ wells tend to yield inaccurate estimation of hydrocarbon pore volume by as much as $15 \%$. This behavior originates from geometrical complications such as differing volumes of investigation in the several measurements and complex relative bed geometry resulting from undulating well trajectories. Furthermore, I conclude that inversion is an efficient procedure to account for such geometrical complications in $\mathrm{HA} / \mathrm{HZ}$ wells.

vii. Data resolution enforced by multi-sector binning and multi-array resistivity plays a significant role in the length of confidence intervals of results obtained with nonlinear inversion and multi-mineral analysis. It influences the degree of rank determination, i.e., under-, even-, or over-determined estimation. Specifically, incorporating multiple measurements such as multi-sector, multi-array, dualfrequency, phase and attenuation resistivities promotes stability of the inverse problem and improves confidence in interpreted properties.

viii. Additionally, in Appendix C, I investigated the effects of resistivity anisotropy and polarization horns on layer-based inversion. By constructing the Jacobian 
matrix with rapid numerical simulation of apparent propagation resistivities, measurement responses to bed boundaries and formation anisotropy were described in the inversion. The calculated confidence intervals were proportional to the degree of non-uniqueness of inverted results whereby I conclude that inverted vertical and horizontal resistivities can be non-unique, especially across thinly bedded formations. This is another limitation of the inversion-based interpretation workflow.

ix. Separate inversion in the nuclear domain is about 11 times faster than in the resistivity domain. For example, inversion interpretation of the horizontal well field case example discussed in Chapter 4 and Figure 4.10 took about 5 minutes in the nuclear domain and 55 minutes in the resistivity domain on a desktop computer. This is because the nuclear Jacobian matrices were directly constructed with 3D FSFs while the conductivity Jacobian matrix required multiple forward simulations to approximate the partial derivatives. This is a limitation of the separate inversion of apparent conductivity measurements as implemented in the dissertation.

\subsubsection{Part Three: Interpretation of Porosity and Fluid Constituents from Well Logs Using an Interactive Neutron-Density Matrix Scale}

i. I introduced a new method for interpreting formation porosity and hydrocarbon density from well logs using a variable and interactive neutron-density matrix scale.

ii. The merits of the new interpretation method include estimation of lithologyindependent and model-consistent porosity, identification of hydrocarbon-bearing sweet spots, and determination of fluid contacts or/and reservoir compartments. 
iii. To calculate matrix effects (grain density, shale-hydroxyls, and matrix-hydrogen) with the SNUPAR-based solver on depth-by-depth neutron and density porosity $\operatorname{logs}$, I assumed water-filled rocks. This assumption is adequate because formation fluids have negligible or no influence on PEF and GR log. Subsequently, the interpretation workflow re-scales the neutron-density overlay with a depth-bydepth variable matrix scale so that fluid effects can be independently inferred from the matrix-corrected neutron and density logs.

iv. The SNUPAR-solver guides the interactive analysis in the two-step quantification of depth-by-depth (1) matrix effects, and (2) fluid densities. In this manner, the Gaymard-Poupon formulation for porosity calculation in clean formations is extended to any neutron-density matrix scale and formation lithology (clean or/and shaly formations).

v. I successfully validated results of the interpretation workflow with field examples and laboratory core measurements. Field examples included gas-saturated carbonate, shale-gas, and gas-/oil-saturated siliciclastic reservoirs drilled with vertical wells. The analysis workflow is reliable for estimating lithologyindependent porosity and hydrocarbon density from conventional nuclear and resistivity logs without expensive acquisition of nuclear magnetic resonance (NMR) measurements.

vi. Matrix-hydrogen effects on neutron porosity logs can mask the interpretation of fluid effects from the neutron-density overlay. The interactive interpretation workflow becomes useful for shaly and organic-rich formations where it delivers depth-variable corrections for shale-hydroxyl and kerogen matrix-hydrogen effects. Its application could be superfluous in limestone reservoirs with 
negligible shale or siltstone content given that the neutron porosity $\log$ is commonly delivered in limestone units.

vii. The interpretation workflow implements depth-by-depth matrix and fluid analysis of well logs with the assumption of negligible shoulder-bed effects. Across highly laminated formations, this assumption is invalid whereby the estimated hydrocarbon density could be inaccurate and unreliable.

\subsection{RECOMMENDATIONS}

The following is a list of recommendations that could advance the research topics documented in this dissertation:

i. The new $F_{p}$ formation parameter could be further enhanced by incorporating other neutron cross-section properties, such as formation diffusion coefficient and hydrogen index, in the empirical parameterization. A hybrid of the $F_{p}$ and DFD techniques could be implemented to further improve the physical description of spatial neutron porosity responses. Moreover, investigating multi-group diffusion models could provide further insights to the quantification of higher-order perturbation terms.

ii. The Green's function approach implemented in the development of the DFD technique could be extended to gamma-gamma density measurements. A gamma ray attenuation model could be implemented as the Green's function kernel to describe a spatial gamma flux-difference function for Compton scattering. This approach could enable new quantitative procedures for improving dual-detector density compensation techniques in the presence of tool standoff and mudcake. Preliminary results indicated that gamma density coefficients calculated for a generic LWD density tool in the same manner as the neutron transport-correction 
coefficients were relatively constant for water- and gas-saturated sandstone, limestone, and dolomite formations, including aluminum and magnesium calibration blocks (refer to Figure 6.2). Furthermore, a preliminary implementation indicated that a first-order Rytov gamma flux-difference function could remain inaccurate for Compton scattering. Instead, a second-order formulation of the Green's function kernels is recommended for quantifying the spatial flux-difference function.

iii. A crucial step of the inversion-based interpretation workflow is the construction of a layer-based geometrical model. Results show that uncertainties in bed boundary location and orientation could yield errors as high as $20 \mathrm{pu}$ in estimated porosity, especially across thinly bedded formations. This is a limitation of the layer-based model as implemented in the dissertation. High resolution borehole images, e.g., formation micro-images or acoustic image measurements, could be used to enhance bed boundary information and decrease uncertainty in the geometrical model. Additionally, well survey data could be incorporated into the workflow to improve well trajectory information, especially in undulating $\mathrm{HA} / \mathrm{HZ}$ wells.

iv. The inversion-based interpretation algorithm is efficient for the assimilation of other LWD borehole measurements, such as acoustic, nuclear elemental spectroscopy, Sigma, and magnetic resonance. Separate inversion ensures the enforcement of multi-physics description through independent fast-forward models within each measurement domain. Assimilating nuclear elemental spectroscopy and Sigma measurements with their respective forward models in the inversion-based workflow could improve convergence of the compositional solver. 
v. The inversion-based method implemented in this dissertation assumes marginal invasion and tool standoff whereby LWD measurements have been adequately compensated or/and are not severely affected by mud-filtrate invasion. When such an assumption is not met, standoff and invasion maps inverted from dual-detector density images and multi-array resistivity measurements along the borehole could be included into the geometrical model. The expanded geometrical model could then be imposed on other available measurement domains for improved description of borehole and environmental effects.

vi. In the resistivity domain of the inversion-based workflow, where computational efficiency is limited by the construction of Jacobian matrices from multiple forward simulations, improvements in speed could be achieved with the implementation of non-gradient based methods. Such methods include Bayesian inversion and derivative-free algorithms (Aster et al., 2005).

vii. The SNUPAR-based compositional solver requires a priori knowledge of matrix and fluid types present in the formations. Preliminary lithology cross-plots, x-ray diffraction data, or/and core measurements could be used to obtain information about primary lithology and saturating-fluids. This a priori knowledge, implemented as an initial guess, could speed up the convergence of the compositional solver.

viii. I recommend an approach to control the importance of inverted layer-by-layer properties input in the SNUPAR-based solver. Weighted importance matrices could be constructed from the confidence intervals calculated from equation B.1 in Appendix B, whereby importance remains inversely proportional to the relative or absolute size of the confidence intervals. 
ix. The interactive matrix analysis assumes that shoulder-bed effects are negligible and depth-by-depth analysis is adequate. Across highly laminated formations with significant shoulder-bed effects, corrections (such as inversion processing) could be applied prior to performing the interactive matrix analysis.

x. The interactive interpretation workflow could be extended to the quantification of sand-shale distribution topologies inferred from Thomas-Stieber cross-plots. This approach will involve implementing SNUPAR to quantify the relationship between sand-shale topologies (laminated, dispersed, or/and structural) and nuclear measurements (GR, density, neutron porosity, and PEF). Ultimately, fitfor-purpose volumetric mixing laws for specific sand-shale topologies could be obtained from the SNUPAR-derived models. 


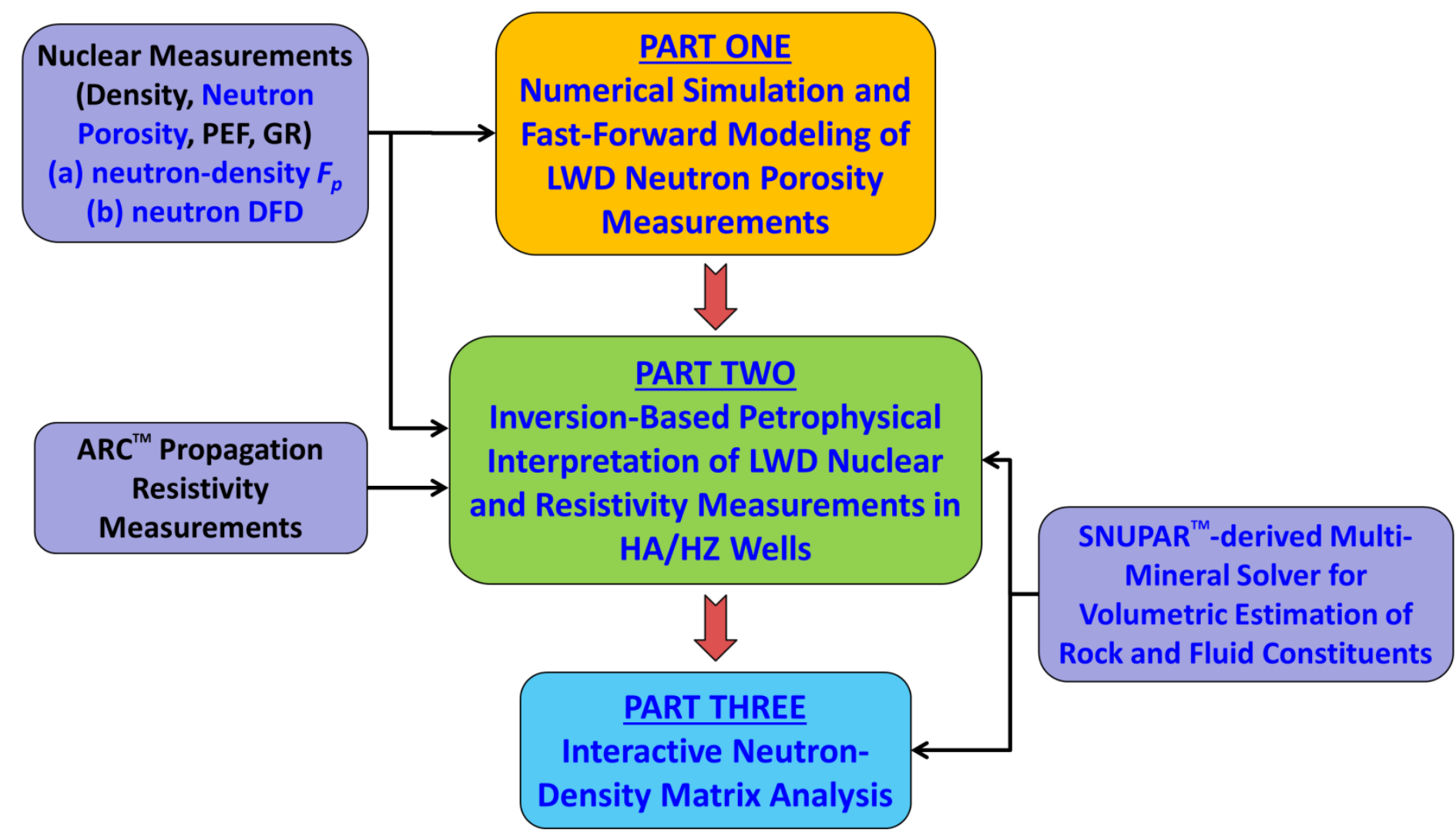

Figure 6.1: Flow chart summarizing the three parts of the dissertation. ARC: Schlumberger Array Resistivity Compensation measurements; SNUPAR: Schlumberger Nuclear Parameter program. 


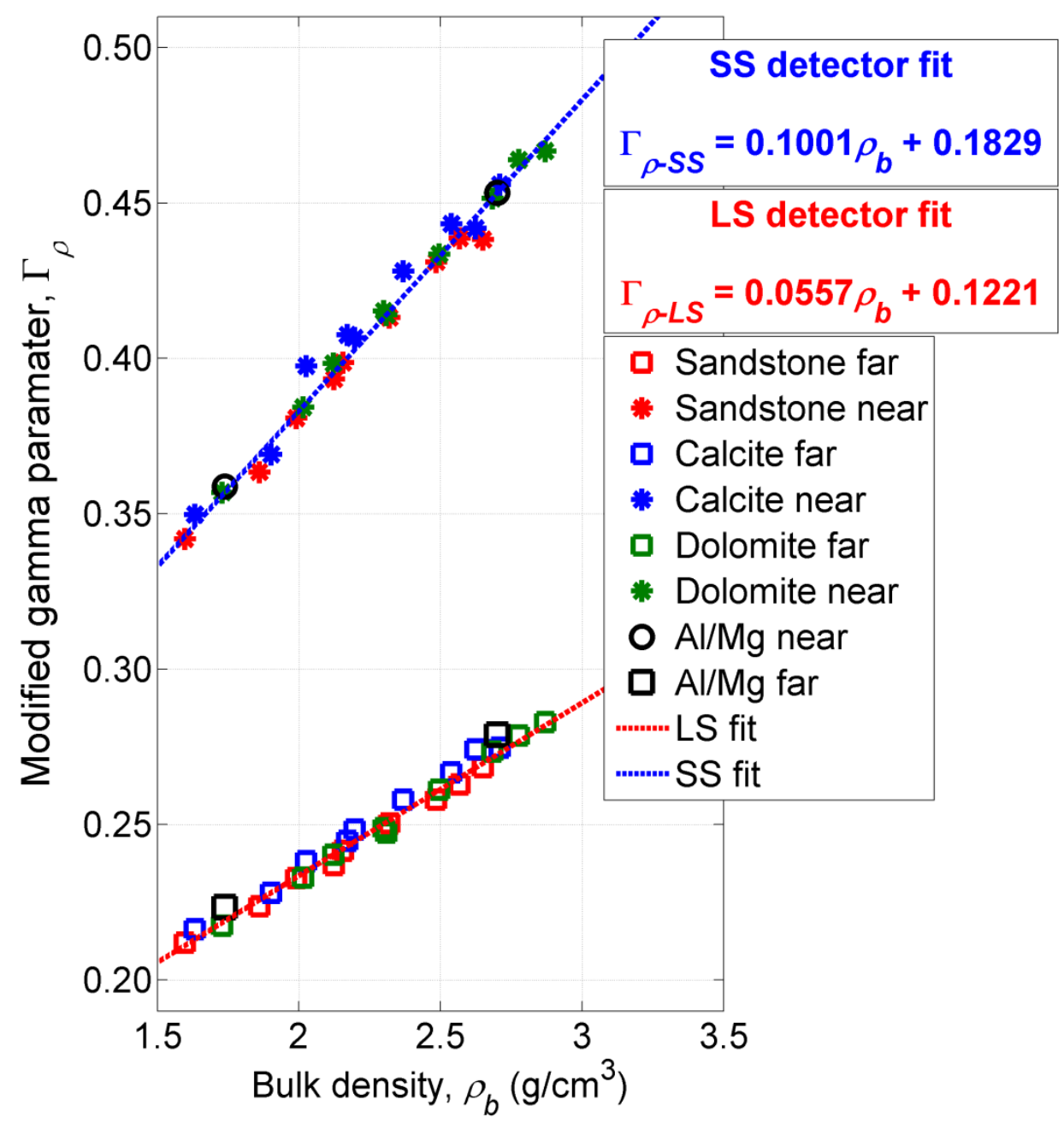

Figure 6.2: Preliminary results toward the development of a gamma flux-difference approximation method for Compton scattering. The y-axis describes the modified gamma parameter (analogous to modified neutron travel length discussed in Chapter 3) for short- (SS) and long-spaced (LS) detectors, while the $\mathrm{x}$-axis describes the formation bulk density; SS and LS detector trend lines indicate constant gamma coefficients for sandstone, limestone, and dolomite formations, including aluminum and magnesium calibration blocks. 


\section{Appendix A: Synthetic Examples of Flux-Difference Modeling of Neutron Porosity Measurements}

\section{A.1 Synthetic Case of a High-Angle Well with Standoff Effects}

The synthetic model described in Table 3.3 with layer TST of $1.3 \mathrm{ft}$ is penetrated with an eccentered LWD tool in a 12-in. water-filled borehole to investigate borehole environmental effects. A maximum standoff of 3.5 in. takes places at the $U$ sector orientation of the borehole. Various degrees of borehole standoff are measured as the tool rotates around the hole. This behavior creates a "railroad" effect typical in eccentered tool logging. Figure A.1 shows the corresponding simulation results. The "railroad" effect is observed in $\phi_{N-L I R}, \phi_{N-D F D}, \phi_{N-M C}$, and $\rho_{C O-L I R}$ (panels b, c, d, and e respectively). Spineand-rib density compensation is rendered ineffective at the top of the hole where radial standoff is 3.5 in. because gamma depth of investigation (DOI) is approximately $3 \mathrm{in}$. (Ijasan et al., 2011). Comparison of bottom sector logs in panels f of Figures 3.11 and A.1 indicate that standoff has a greater effect on neutron logs than on density logs. Density porosity increases by $1 \mathrm{pu}$, while neutron porosity increases by as much as $6 \mathrm{pu}$ in the shale layers and 1 pu in gas-bearing layers. Panel i of Figure A.1 shows average porosity errors obtained with $\phi_{N-L I R}$ and $\phi_{N-D F D}$ in comparison to $\phi_{N-M C}$, where I observe that $\phi_{N-D F D}$ yields consistently lower porosity errors than $\phi_{N-L I R}$.

\section{A.2 Borehole Standoff Effects in Wireline Measurements}

Despite acquisition of wireline measurements with pad tools, standoff is still prevalent, especially in washed-out boreholes. The 2D DFD functionality is implemented for the case of wireline standoff perturbations. Figure A.2 qualitatively compares 1D FSF vertical and radial projections of the wireline neutron sonde in a 12-in. borehole across water- and gas-filled limestone formation base-cases using MCNP, DFD, and LIR methods. The MC-derived background FSFs are generated in an 8-in. borehole, with 
sonde pressed against the borehole, across base-cases of the library set described in Table 3.2. Under these conditions, detector responses are predicted across a 2 -in. standoff. In Figure A.2, I observe that $F S F_{L I R}$ matches $F S F_{M C}$ in the standoff region only (panels b and d). On the other hand, the DFD approximation agrees with $F S F_{M C}$ in both standoff and formation regions. Similar observations are made about the vertical projections of panels a and c. The perturbed $F S F_{D F D}$, shown in Figure A.2, is obtained through perturbation about homogeneous background base-cases (as in equation 3.6) such that the calculated flux perturbation is due to standoff only. For example, $F S F_{D F D} 12 \mathrm{BH}$ LIME05W, i.e., perturbed FSF response across a water-filled $5 \%$ porosity limestone formation with 2-in. standoff, is calculated using 2D DFD models (equations 3.3 - 3.6) and $F S F_{B} 08 \mathrm{BH}$ LIME05W, i.e., MC-derived homogeneous FSF response with sonde pressed against the borehole.

Figure A.3 shows calculated detector counts and $\phi_{N}$ errors, in comparison to MCNP, of LIR and DFD methods. I observe that LIR is not accurate to quantify flux perturbations due to standoff, thereby resulting in larger $\phi_{N}$ errors than with the DFD. These errors are especially severe at low porosities and across gas-filled formations because of large borehole-formation contrasts. Above $20 \mathrm{pu}$ formation porosity, where borehole-formation contrasts decrease, LIR yields acceptable errors lower than $1 \mathrm{pu}$. The DFD method is accurate to less than $1 \mathrm{pu}$ error in the presence of 2-in. standoff across limestone formations of varying water and gas-filled porosities (Figure A.3, panels a and b) while LIR yields up to $7 \mathrm{pu}$ error in gas-filled formations. Similar standoff perturbations are repeated for 1-in. and 3.5-in. standoff sizes. These standoff sizes correspond to 10-in. and 15-in. borehole sizes, respectively. The significant LIR $\phi_{N}$ errors are due to inadequacy of first-order perturbation to quantify standoff effects. These errors are proportional to standoff size and borehole-formation contrast (Figure A.3, panel c). 


\section{A.3 Synthetic Case of a High-Angle Well with Thin Bed Effects}

To investigate thin bed effects, TST in the synthetic model of Table $\mathbf{3 . 3}$ is decreased from $1.3 \mathrm{ft}$ to $0.49 \mathrm{ft}$, and penetrated with the LWD tool in a slick borehole. Shoulder-bed effects and formation variability increase when decreasing the layer thickness. With this model, I confirm the accuracy and robustness of the 3D DFD method for applications in thinly bedded formations. Figure A.4 shows the $\phi_{N-M C}, \phi_{N-D F D}$, and $\phi_{N-}$ LIR logs in sandstone pu. In comparison to Figure 3.11, false gas crossover effects in layer IV are accentuated from $15 \mathrm{pu}$ to $22 \mathrm{pu}$ due to increased shoulder-bed effects (panel f). Similar to the observations previously made about Figure 3.11, LIR does not accurately reproduce the spatial distribution of perturbed FSFs, thereby resulting in $\phi_{N-L I R}$ errors as large as 5 pu. Moreover, because perturbed FSFs obtained with the DFD technique agree better with MCNP FSFs, $\phi_{N-D F D}$ simulation errors are minimal. 
(a) Formation model in $L_{m}$
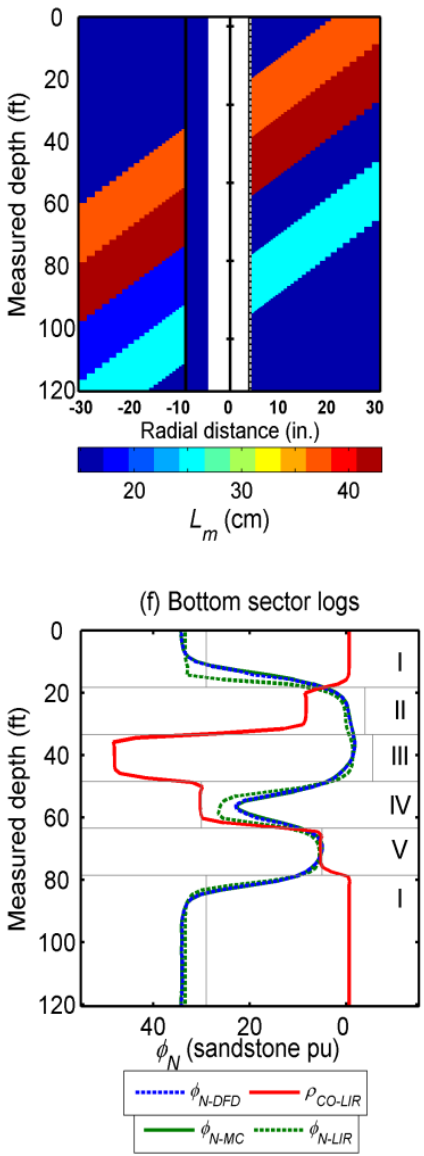

(b) $\phi_{N-L I R}$

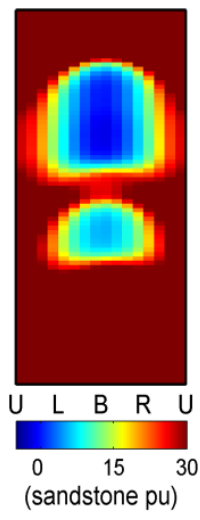

(g) $\phi_{N-M C}-\phi_{N-L R}$

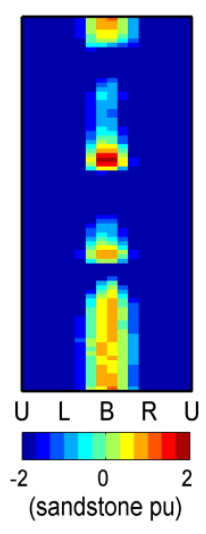

(c) $\phi_{N-D F D}$

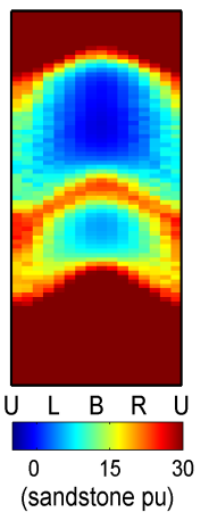

(h) $\phi_{N-M C^{-}} \phi_{N-D F D}$

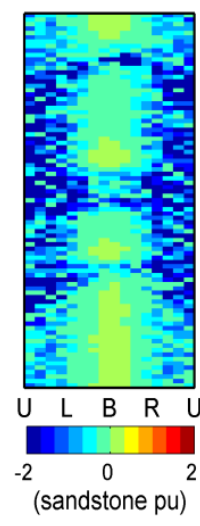

(d) $\phi_{N-M C}$
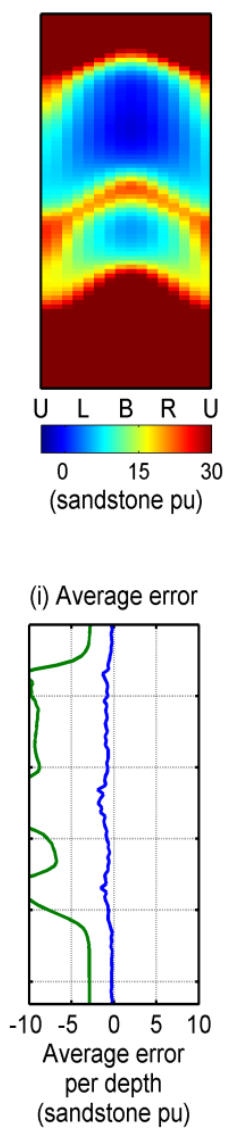

(e) $\rho_{\text {CO-LIR }}$
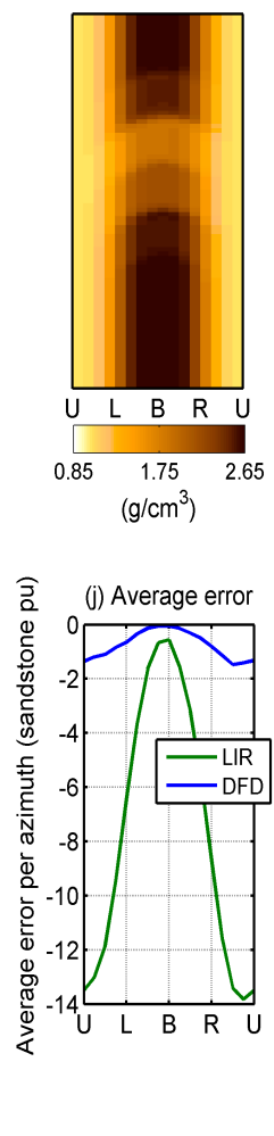

Figure A.1: Comparisons of LWD neutron porosities $\phi_{N-M C}, \phi_{N-D F D}$, and $\phi_{N-L I R}$, in sandstone pu, simulated with MCNP, DFD, and LIR methods, respectively, in a 12-in. water-filled borehole for the synthetic model described in Table 3.3 where TST is $1.3 \mathrm{ft}(40 \mathrm{~cm})$. (a) Description of the layered earth model in $L_{m}$ (not to scale), (b) neutron porosity, $\phi_{N-L I R}$ calculated with the LIR method, (c) neutron porosity, $\phi_{N-D F D}$ calculated with the DFD method, (d) neutron porosity, $\phi_{N-M C}$ rendered with full MCNP calculations, (e) compensated density $\rho_{C O-L I R}$ calculated with LIR, (f) bottom sector neutron and density logs (layer descriptions included in Table 3.3), (g) LWD porosity residuals, $\phi_{N-M C}-\phi_{N-L I R}$, (h) LWD porosity residuals, $\phi_{N-M C}-\phi_{N-D F D}$, (i) average error per depth, and (j) average error per azimuth; DFD calculations were performed by invoking their 3D kernel functionality. 
(a)

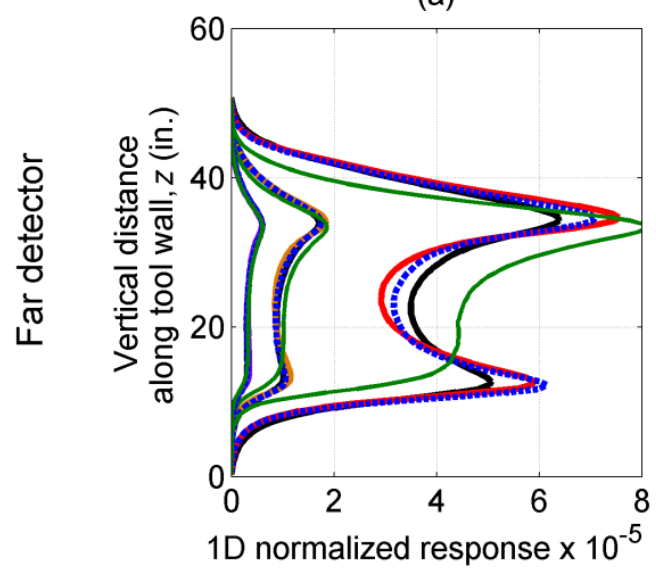

(c)

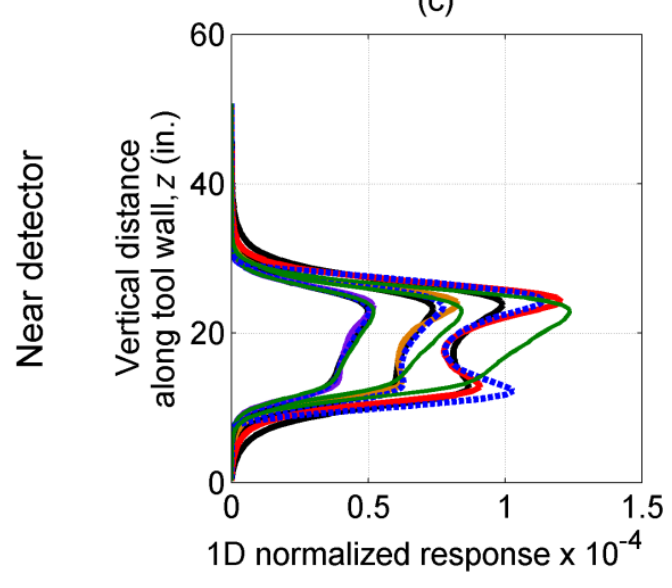

(b)
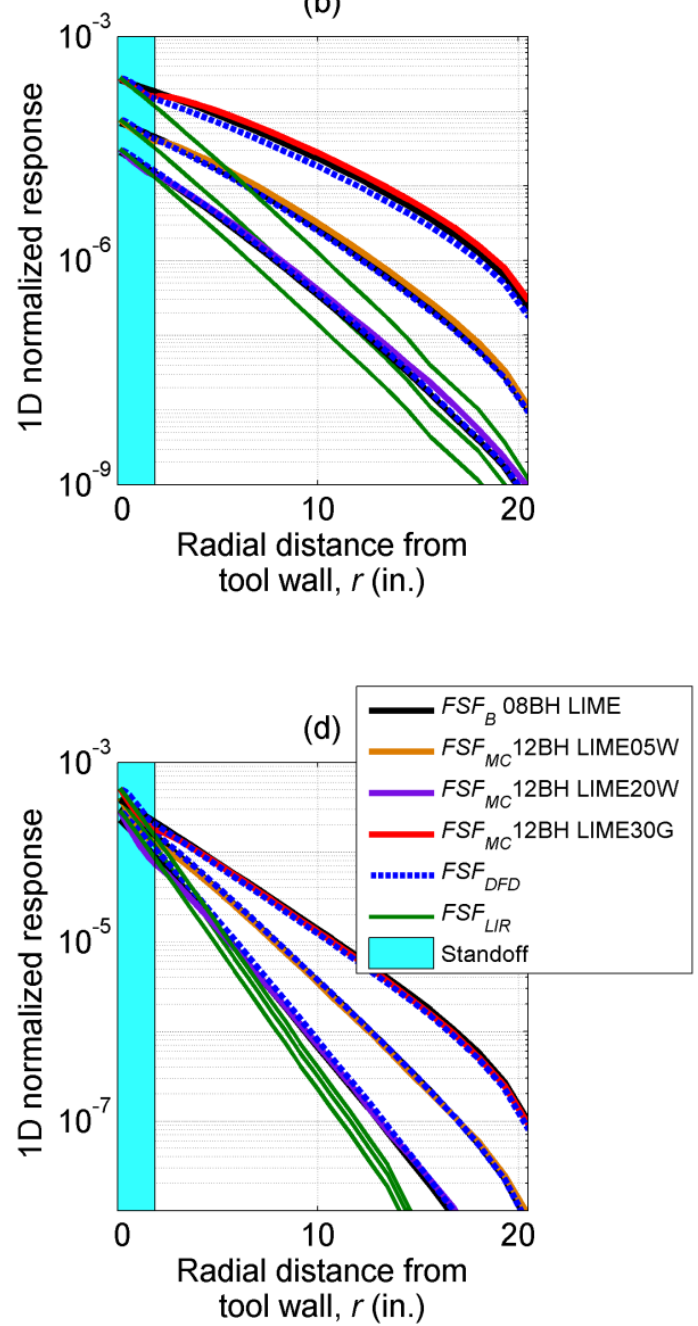

Figure A.2: Qualitative comparisons of the DFD method to full MCNP calculations across water- and gas-filled limestone formations, in the presence of 2-in. standoff for wireline sonde. Left and right panels show 1D FSF vertical and radial projections, respectively. In this case, the $2 \mathrm{D}$ kernel functionality is used for DFD calculations of the wireline tool. (a) Far detector FSF vertical projections, integrated in the radial and azimuthal directions, showing background MC-derived $F S F_{B}$ with solid black lines, MC-derived perturbed $F S F_{M C}$ in solid colored lines, corresponding DFD perturbed $F S F_{D F D}$ with a dashed blue line, and equivalent $F S F_{L I R}$ with solid green lines. (b) Far detector FSF radial projections, integrated in the vertical and azimuthal directions, showing the standoff region. (c) Similar to (a) but for the near detector. (d) Similar to (b) but for the near detector. The borehole is assumed to be filled with fresh water. 
(a)

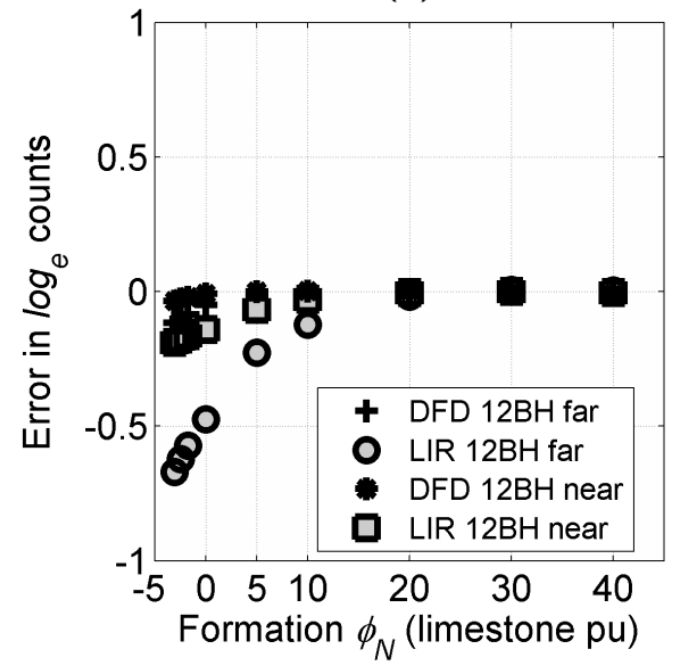

(b)

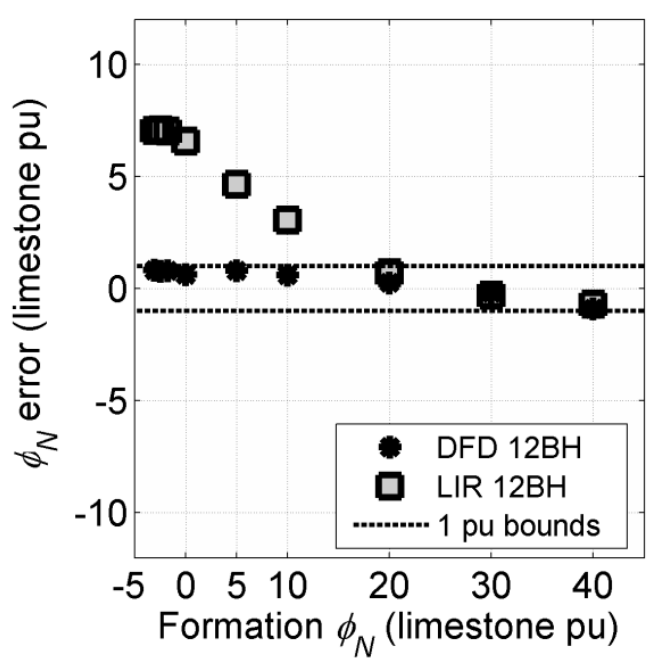

(c)

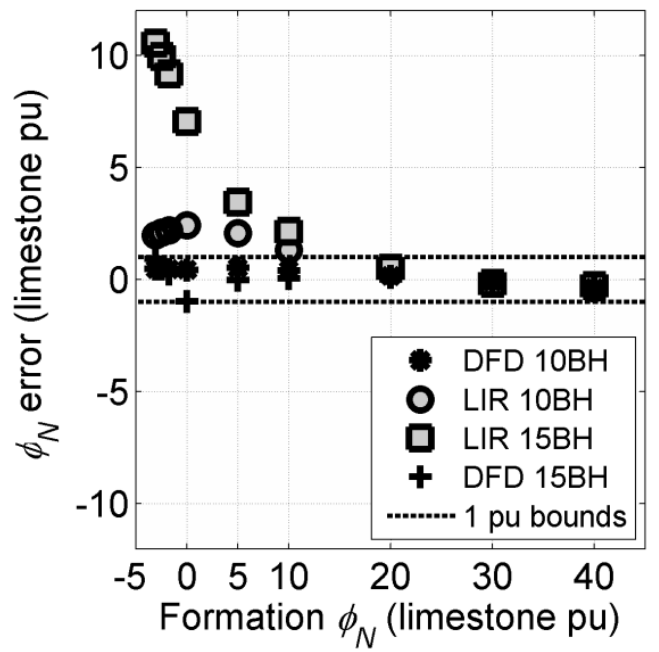

Figure A.3: Quantitative comparisons of wireline $\phi_{N}$ calculated with DFD and LIR methods against MCNP. (a) Error in far and near detector counts versus formation $\phi_{N}$ in a 12-in. borehole. (b) $\phi_{N}$ errors in a 12 -in. borehole. (c) $\phi_{N}$ errors in 10-in. and 15-in. boreholes. DFD calculations were performed by invoking their 3D kernel functionality. The borehole is assumed to be filled with fresh water. 
(a) Formation model in $L_{m}$
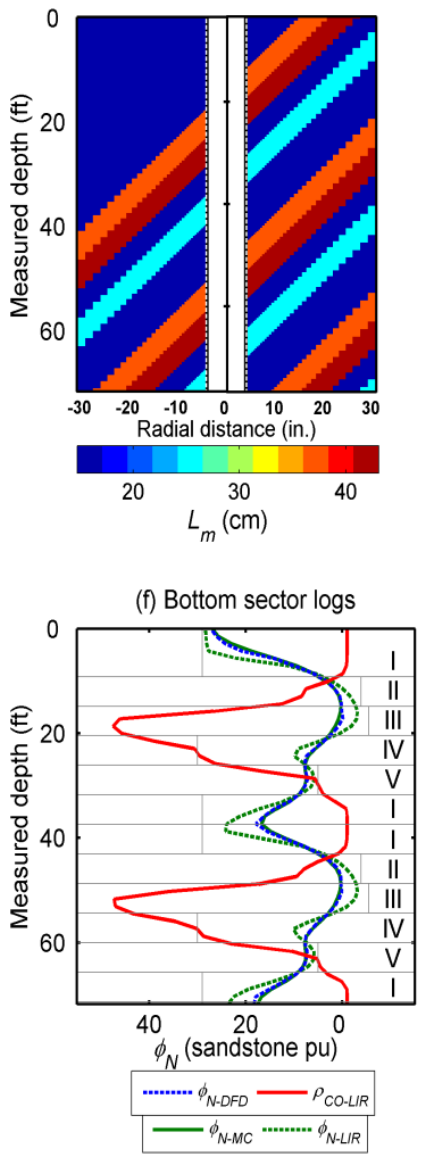

(b) $\phi_{N-L I R}$

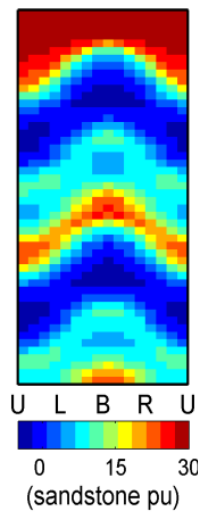

(g) $\phi_{N-M C}-\phi_{N-L I R}$

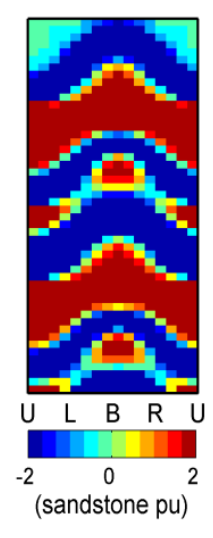

(c) $\phi_{N-D F D}$

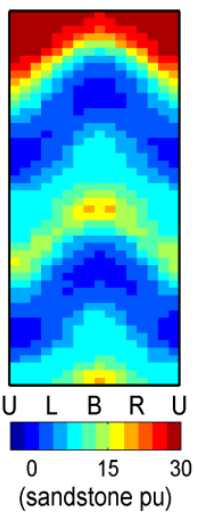

(h) $\phi_{N-M C}-\phi_{N-D F D}$

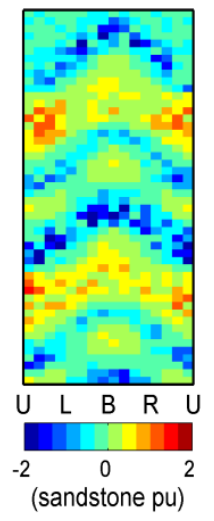

(d) $\phi_{N-M C}$

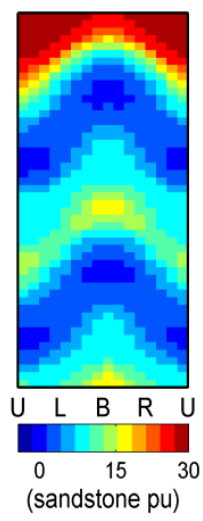

(i) Average error

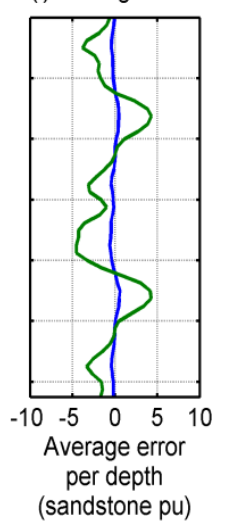

(e) $\rho_{\text {CO-LIR }}$
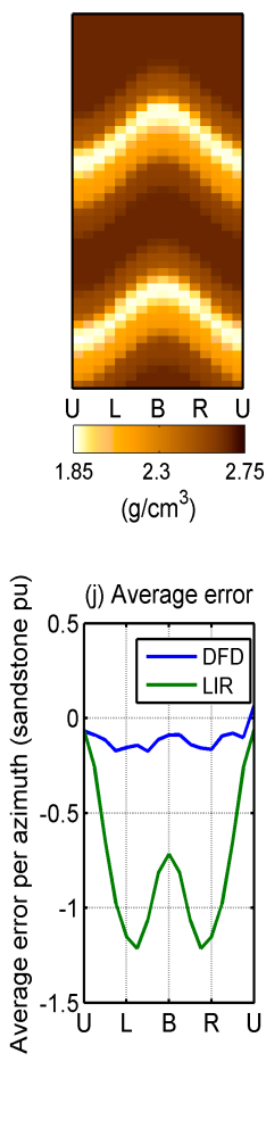

Figure A.4: Comparisons of LWD neutron porosities $\phi_{N-M C}, \phi_{N-D F D}$, and $\phi_{N-L I R}$, in sandstone pu, simulated with MCNP, DFD, and LIR methods, respectively, in a slick borehole for the synthetic model described in Table 3.3 where TST is $0.49 \mathrm{ft}(15 \mathrm{~cm})$. (a) Description of the layered earth model in $L_{m}$ (not to scale), (b) neutron porosity, $\phi_{N-L I R}$ calculated with the LIR method, (c) neutron porosity, $\phi_{N-D F D}$ calculated with the DFD method, (d) neutron porosity, $\phi_{N-M C}$ rendered with full MCNP calculations, (e) compensated density $\rho_{C O-L I R}$ calculated with LIR, (f) bottom sector neutron and density logs (layer descriptions included in Table 3.3), (g) LWD porosity residuals, $\phi_{N-M C}-\phi_{N-L I R}$, (h) LWD porosity residuals, $\phi_{N-M C}-\phi_{N-}$ $D F D$, (i) average error per depth, and (j) average error per azimuth; DFD calculations were performed by invoking their 3D kernel functionality. 


\section{Appendix B: Computational Tools for Inversion of Nuclear and Resistivity Measurements}

\section{B.1 Nonlinear Separate Inversion in LWD Measurement Domains}

I implement the Levenberg-Marquardt iterative nonlinear minimization method (Aster et al., 2005; Hansen, 1998) in each measurement domain, as shown in equation 4.3 , such that $95 \%$ confidence intervals for each inverted petrophysical parameter are calculated with

$$
\mathbf{p} \pm 1.96 \sqrt{\|\mathbf{e}(\mathbf{p})\|_{2}^{2} \cdot \operatorname{diag}\left(\mathbf{J}^{T} \mathbf{J}\right)^{-1}}
$$

where the superscript $T$ designates matrix transpose.

\section{B.1.1 Data and Parameter Space}

In each measurement domain, the layer-by-layer inverted petrophysical parameter is given by

$$
\mathbf{p}=\left[p_{1}, \cdots, p_{b}, \cdots, p_{n_{b}}\right]^{T}
$$

while data are written as

$$
\mathbf{d}_{s}=\left[d_{1}, \cdots, d_{s}, \cdots, d_{n_{s p}}\right]^{T}
$$

where $s$ and $b$ designate the $s$-th available data and $b$-th layer, respectively, while $n_{s p}$ is total number of available data points and $n_{b}$ is total number of layers. In nuclear domain, $n_{s p}$ is $J \times M \times n_{\text {det }}$, where $n_{\text {det }}$ is number of detectors, $J$ is number of azimuthal sector bins, 
and $M$ is number of sampled depth points. For example, $J=1$ for single curves of PEF and GR logs, while $J=16$ for 16 -sector binning scheme. Similarly, $n_{\mathrm{det}}=1$ for $\rho_{C O}$ and $\phi_{N}$, while $n_{\text {det }}=2$ for dual-detector density ( $\left.\rho_{S S}, \rho_{L S}\right)$ and neutron (ncps, fcps) measurements. In resistivity domain, $n_{s p}$ is $20 \times M$, i.e., 4 channels each (phase, attenuation, and dual frequencies) for 5 array (16-, 22-, 28-, 34-, and 40-in.) propagation measurements. In total, number of measurement domains, $n_{m d}$, is 5 , i.e., density, neutron porosity, PEF, GR, and resistivity.

\section{B.1.2 Numerical Construction of Operator Matrices}

In the nuclear measurement domain, entries of $\mathbf{J}$ are constructed directly from 3D FSFs, as follows:

$$
\mathbf{J}_{s d}=\left[\begin{array}{ccccc}
\mathrm{FSF}_{1,1,1}^{s d} & \cdots & \mathrm{FSF}_{1,1, b}^{s d} & \cdots & \mathrm{FSF}_{1,1, n_{b}}^{s d} \\
\vdots & \ddots & \vdots & \ddots & \vdots \\
\mathrm{FSF}_{1, m, 1}^{s d} & \cdots & \mathrm{FSF}_{1, m, b}^{s d} & \cdots & \mathrm{FSF}_{1, m, n_{b}}^{s d} \\
\vdots & \ddots & \vdots & \ddots & \vdots \\
\mathrm{FSF}_{1, M, 1}^{s d} & \cdots & \mathrm{FSF}_{1, M, b}^{s d} & \cdots & \mathrm{FSF}_{1, M, n_{b}}^{s d} \\
\vdots & \ddots & \vdots & & \vdots \\
\vdots & \ddots & \mathrm{FSF}_{j, m, b}^{s d} & \ddots & \vdots \\
\vdots & & \vdots & \ddots & \vdots \\
\mathrm{FSF}_{j, M, 1}^{s d} & \cdots & \mathrm{FSF}_{j, M, b}^{s d} & \cdots & \mathrm{FSF}_{j, M, n_{b}}^{s d} \\
\vdots & \ddots & \vdots & \ddots & \vdots \\
\vdots & \ddots & \vdots & \ddots & \vdots \\
\mathrm{FSF}_{J, M, 1}^{s d} & \cdots & \mathrm{FSF}_{J, M, b}^{s d} & \cdots & \mathrm{FSF}_{J, M, n_{b}}^{s d}
\end{array}\right]
$$

where superscript/subscript $s d$ designates single-detector nuclear measurement (e.g., PEF and GR), subscripts $j, m$, and $b$ designate $j$-th sector-based bin up to $J$ azimuthal orientations, $m$-th measurement point (along the well trajectory) up to $M$ depth points, 
and $b$-th layer up to $n_{b}$ layers, respectively. In order words, $\mathrm{FSF}_{j, m, b}^{s d}$ is the sensitivity of a single-detector well log measurement at $m$-th depth point and $j$-th azimuth to $b$-th layer. It follows from equation B.4 that the Jacobian matrix for dual-detector measurements (gamma densities and neutron count rates), $\mathbf{J}_{d d}$, is obtained from $\mathbf{J}_{s d}$ of short-spaced (SS)/near and long-spaced (LS)/far detectors for density/neutron measurements, i.e.

$$
\mathbf{J}_{d d}=\left[\mathbf{J}_{s d, S S / \text { near }}, \mathbf{J}_{s d, L S / f a r}\right]^{T}
$$

To account for flux perturbations in neutron porosity measurements acquired in $\mathrm{HA} / \mathrm{HZ}$ wells, I implement the semi-analytical transport-diffusion approximation in Chapter 3 (Ijasan et al., 2013) such that $\mathbf{J}_{d d}$, for dual-detector neutron count rates, becomes

$$
\mathbf{J}_{d d}=\left[\mathbf{J}_{s d, \text { near }}, \mathbf{J}_{s d, f a r}\right]^{T}+\left[\mathbf{J}_{D F D, \text { near }}, \mathbf{J}_{D F D, f a r}\right]^{T},
$$

where $\mathbf{J}_{D F D}$ is the DFD approximation for neutron near and far detectors, accordingly. In equation B.6, the first term on the right-hand-side describes the transport part, while the second term describes the diffusion part of $\phi_{N}$ measurement physics.

\section{B.1.3 Finite-Difference Approximation of Operator Matrices}

Entries of Jacobian matrices, $\mathbf{J}_{C O}$ and $\mathbf{J}_{\sigma}$ for inversion of compensated nuclear $\left(\rho_{C O}, \phi_{N}\right)$ and apparent electrical conductivity measurements $\left(1 / R_{\text {app }}\right)$, respectively, are obtained by finite-difference approximations of partial derivatives using rapid numerical simulations of well-log data, $\mathbf{d}(\mathbf{p})$. In the nuclear measurement domain, entries of $\mathbf{J}_{C O}$ are numerically calculated from equations B.5, B.6, and appropriate post-processing 
algorithm, i.e., density spine-and-rib compensation and neutron near-to-far ratio porosity, for $\rho_{C O}$ and $\phi_{N}$, respectively. Similarly, elements of $\mathbf{J}_{\sigma}$ for inversion of apparent electrical conductivity measurements $\left(1 / R_{\text {app }}\right)$ are obtained from perturbations in $\mathbf{d}(\mathbf{p})$ due to perturbations in true layer conductivities, $\mathbf{p}\left(\sigma_{t}\right)$. It follows that effects of resistivity anisotropy and polarization horns are inherent in numerically simulated $R_{a p p}$, and consequently in $\mathbf{J}_{\sigma}$.

\section{B.2 SNUPAR-Based Compositional Solver}

The SNUPAR-based compositional solver (Heidari et al., 2012) calculates solid and fluid volumetric concentrations from inverted layer-by-layer petrophysical parameters, p, using Occam's inversion method (Aster et al., 2005). The minimized quadratic cost function is expressed as

$$
\mathbf{C}(\mathbf{x})=\left\|\mathbf{p}_{\text {snu }}(\mathbf{x})-\mathbf{p}\right\|_{2}^{2}+\alpha^{2}\left\|\mathbf{x}-\mathbf{x}^{0}\right\|_{2}^{2}
$$

where $\mathbf{x}=\left[V_{1}, \cdots, V_{n}, \cdots, V_{N}\right]^{T}$ is vector of volumetric concentrations, $V_{n}$ is volumetric concentration of $n$-th solid or fluid component for up to $N$ components subject to $0 \leq V_{n} \leq 1 \quad$ and $\quad \sum_{n=1}^{N} V_{n}=1 \forall n, \quad \mathbf{p}=\left[\begin{array}{llll}\rho_{b} & 1 / L_{m} & U_{e} C_{s h} \sigma_{t}\end{array}\right]^{T}$ is inverted layer-by-layer petrophysical parameters, $U_{e}$ is volumetric PEF (a product of $\rho_{b}$ and PEF), $\mathbf{p}_{s n u}(\mathbf{x})$ is SNUPAR-calculated petrophysical parameters given an arbitrary initial guess, $\mathbf{x}^{0}$, of volumetric concentrations, and $\alpha$ is a regularization (stabilization) parameter calculated with the GCV method (Hansen, 1998). The ensuing constrained nonlinear minimization function is given by

$$
\left(\mathbf{J}_{\text {snu }}{ }^{T} \mathbf{J}_{\text {snu }}+\alpha^{2} \mathbf{I}\right) \mathbf{x}^{k+1}=\mathbf{J}_{\text {snu }}{ }^{T} \hat{\mathbf{p}}\left(\mathbf{x}^{k}\right)+\alpha^{2} \mathbf{I} \mathbf{x}^{k},
$$


where $\mathbf{J}_{s n u}$ is a Jacobian matrix of approximate partial derivatives obtained by finitedifference SNUPAR calculations of $\mathbf{p}_{s n u}(\mathbf{x}), \hat{\mathbf{p}}\left(\mathbf{x}^{k}\right)$ is modified misfit vector, $k$ designates the $k$-th iteration, and $\mathbf{I}$ is identity matrix. The Jacobian matrix, $\mathbf{J}_{s n u}$, is derived using

$$
\mathbf{J}_{s n u}=\left[\begin{array}{llllll}
\frac{\partial \rho_{b}}{\partial \mathbf{x}} & \frac{\partial\left(1 / L_{m}\right)}{\partial \mathbf{x}} & \frac{\partial U_{e}}{\partial \mathbf{x}} & \frac{\partial C_{s h}}{\partial \mathbf{x}} & \frac{\partial \sigma_{t}}{\partial \mathbf{x}} & \mathbf{1}
\end{array}\right]^{T}
$$

while the modified misfit vector, $\hat{\mathbf{p}}\left(\mathbf{x}^{k}\right)$, is expressed as

$$
\hat{\mathbf{p}}\left(\mathbf{x}^{k}\right)=\left[\begin{array}{c}
\mathbf{p}-\mathbf{p}_{s n u}\left(\mathbf{x}^{k}\right) \\
0
\end{array}\right]+\mathbf{J}_{s n u} \mathbf{x}^{k},
$$

where the unity-vector and zero entries in equations B.9 and B.10, respectively, minimize the quadratic function $\varepsilon^{2}=\left(\sum_{n=1}^{N} V_{n}-1\right)^{2}$ in a least-square manner. Positivity constraints are enforced on the volumetric concentrations by resetting negative solutions to their previous values in the sequence of iterations and also renormalizing them so that $\sum_{n=1}^{N} V_{n}=1$. In equation B.9, the nuclear derivatives are numerically obtained with SNUPAR calculations and a GR- $C_{s h}$ relationship (e.g., linear; Larionov, 1969; Stieber, 1970; Clavier et al., 1971; etc.), whereas the conductivity derivative is obtained with an appropriate $S_{w}$ model (e.g., Archie, Poupon-Leveaux) assuming known values of Archie's parameters, connate water resistivity, $R_{w}$, shale resistivity, $R_{s h}$, and shale porosity, $\phi_{s h}$. The constrained minimization formulation, equations B.7 to B.10, and the described 
procedure ensure positivity and unity summation of volumetric concentrations, $V_{n}$. Additionally, to improve uniqueness for any arbitrary $\mathbf{x}^{0}, N \leq n_{m d}$. After convergence of the solver, $V_{n}$ is recalculated as $V_{n} / 1+\varepsilon \forall n, \mathbf{x}^{k+1}$ from equation B.8 is output, and confidence intervals from the nonlinear petrophysical inversion (equations 4.3 and B.1) are propagated to estimate uncertainties on solid and fluid concentrations. For the cases studied in this chapter, $\left\|\mathbf{p}_{s m}(\mathbf{x})-\mathbf{p}\right\|_{2}^{2} \leq 10^{-3}$ and $\varepsilon \leq 10^{-4}$ after convergence. Finally, $\rho_{g}$, $\phi_{T}, S_{w}$, and $H P V$ are calculated from $\mathbf{x}^{k+1}$ and the mineral/fluid composition models. 


\section{Appendix C: Effects of Formation Anisotropy and Polarization Horns on Layer-Based Inversion of Propagation Resistivity Measurements}

In practice, formations exhibit electrical anisotropy due to sequence of alternating laminations of dissimilar conductivities, e.g., sand/shale or fine/coarse-grained layers. Anisotropy due to isotropic lamina sequence is often referred to as macroscopic anisotropy, where the vertical and horizontal resistivities are obtained by equivalent series and parallel resistivities, respectively (Chemali et al., 1987; Hagiwara, 1997). Typically, in the presence of formation anisotropy, attenuation resistivity curves are lower than phase-shift resistivity curves. Additionally, polarization horns of unrealistic resistivity values appear at layer boundaries when the induced eddy currents are forced across dipping beds of differing formation conductivities. These polarization horns serve as distinct features for interpreting bed boundaries and constraining inversion uniqueness. Resistivity anisotropy and polarization horns become accentuated across layers with relative bed dip greater than $60^{\circ}$ (Guzmán-Garcia, 2002). This section describes assessment of electrical anisotropy from layer-based inversion of resistivity measurements.

\section{C.1 Synthetic Example}

Panels a and b of Figure C.1 show numerically simulated propagation resistivity measurements, contaminated with $5 \%$ noise in an $82^{\circ}$ well, across the synthetic model in Tables 4.1 and 4.2 but with two times the layer stratigraphic thicknesses. Table C.1 describes electrical anisotropy properties assumed in this synthetic example. I observe that attenuation measurements are lower than phase-shift measurements and $2 \mathrm{MHz}$ array curves exhibit significant separation, thus describing indicative characteristics of resistivity anisotropy. Additionally, polarization horns are evident at layer bed boundaries 
where $R_{a p p}$ exhibits unrealistically high values. Vertical, $\sigma_{v}$, and horizontal, $\sigma_{h}$, conductivities within the designated layers are obtained from inversion of shallow and deep (16- and 40-in. arrays, respectively) apparent resistivity curves, i.e., a total of 8 channels. Subsequently, true electrical conductivities of the anisotropic layers, $\sigma_{t}$, are derived from inverted $\sigma_{v}$ and $\sigma_{h}$. Equations C.1 and C.2 summarize the calculations for assessment of formation anisotropy,

$$
\begin{gathered}
\sigma_{t}=\sqrt{\sigma_{v} \sigma_{h}}, \\
\lambda_{r}=\sqrt{\frac{\sigma_{h}}{\sigma_{v}}},
\end{gathered}
$$

where $\lambda_{r}$ is the layer anisotropy coefficient. Note that in the numerical construction of $\mathbf{J}_{\sigma}$ for assessment of electrical anisotropy, $\sigma_{v}$ and $\sigma_{h}$ are independently perturbed for finitedifference approximations of the partial derivatives, where $\mathbf{p}=\mathbf{p}\left(\sigma_{v}, \sigma_{h}\right)$. In this manner, formation anisotropy is inherently described in $\mathbf{J}_{\sigma}$.

Panels c and d of Figure C.1 compare layer-by-layer inverted resistivities with the true model, while panel e describes layer-by-layer $\lambda_{r}$ calculated with equation C.2. The calculated $R_{t}$ and $\lambda_{r}$ from inversion agree well with model properties in Table C.1. The inversion confidence intervals in panel $\mathrm{d}$, describing degree of stability and nonuniqueness in the minimization, cover the true layer resistivities and are particularly accentuated across the thin beds. In the presence of electrical anisotropy, calculated $R_{t}$ from layer-based inversion is particularly non-unique across thinly-bedded formations. 


\section{C.2 Field Case Example}

The assessment of electrical anisotropy, as discussed above, is implemented across a HA interval in the West Africa field case of study considered in Chapter 4. Figure C.2 shows field and numerically simulated nuclear and resistivity measurement after inversion-based interpretation. In panel $\mathrm{g}, \lambda_{r}$ calculated from equation C.2 range between 1 and 2. Panel $\mathrm{f}$ describes inverted layer-by-layer $R_{v}$ and $R_{h}$ across the alternating clean and silty/shaly layers of TSTs ranging from 1 to $2 \mathrm{ft}$. It also shows calculated $R_{t}$ from equation C.1. Note the confidence intervals describe stability and uniqueness of the inversion for the field example. Inverted $R_{h}$ is more sensitive to the low-resistivity shaly layers, while inverted $R_{v}$ responds to high-resistivity clean layers. Thus, in the estimation of hydrocarbon pore volume, $R_{v}$ is more appropriate than $R_{t}$ in hydrocarbon saturated layers while $R_{h}$ is better suited for the shaly layers. 
Table C.1: Electrical anisotropy properties assumed in the synthetic example.

\begin{tabular}{|c|c|c|}
\hline Layer & $\begin{array}{c}\text { True resistivity, } \\
\boldsymbol{R}_{\boldsymbol{t}}(\Omega-\mathrm{m})\end{array}$ & $\begin{array}{c}\text { Resistivity } \\
\text { anisotropy } \\
\text { coefficient, } \lambda_{\boldsymbol{r}}\end{array}$ \\
\hline $\begin{array}{c}\text { I, IV, VII, X, } \\
\text { XIII }\end{array}$ & 3.54 & 3 \\
\hline $\begin{array}{c}\text { II, V, VIII, } \\
\text { XI }\end{array}$ & 10 & 3 \\
\hline $\begin{array}{c}\text { II, VI, IX, } \\
\text { XII }\end{array}$ & 1.27 & 2 \\
\hline
\end{tabular}




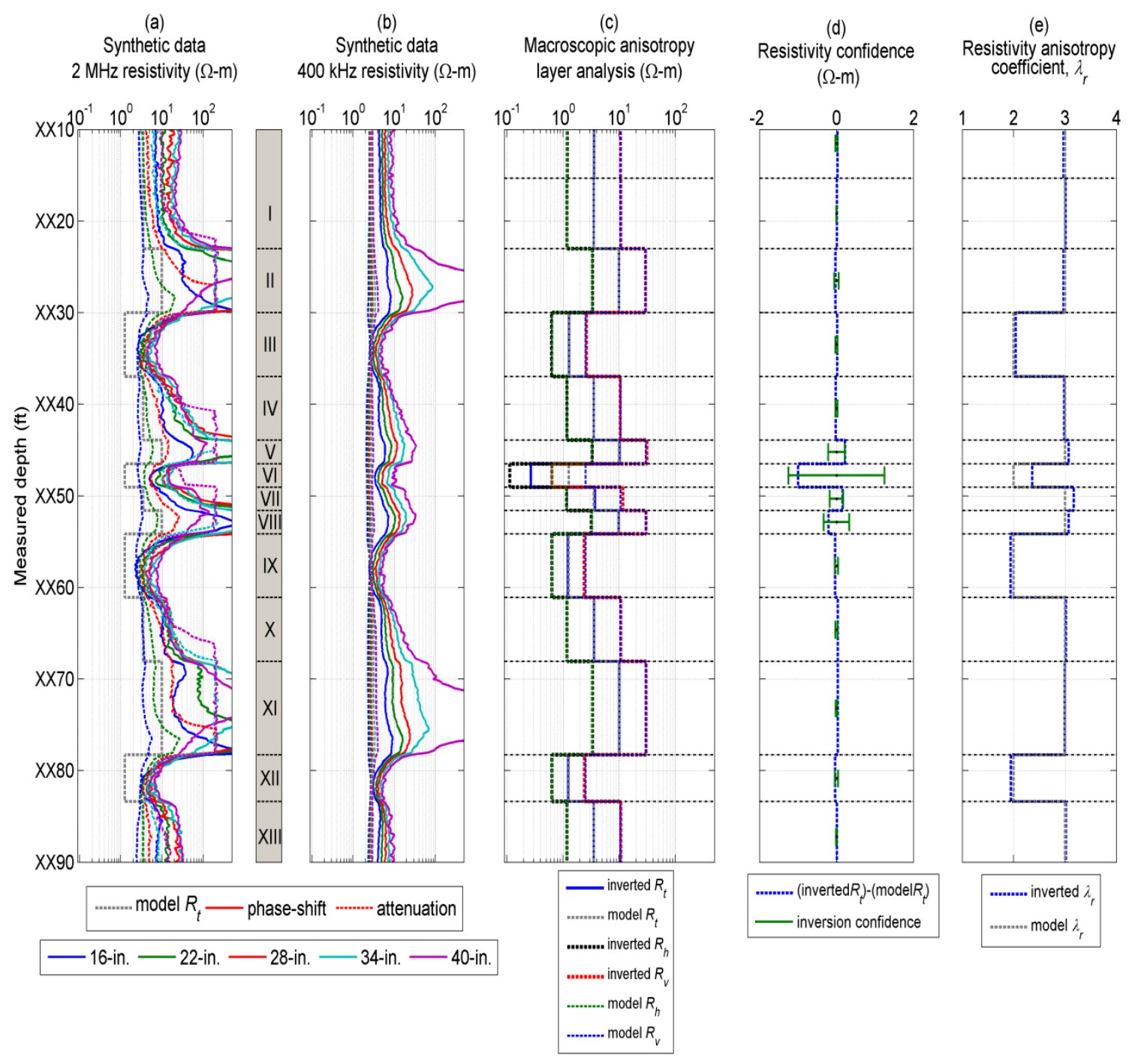

Figure C.1: Effects of resistivity anisotropy and polarization horns on inversion of propagation resistivity measurements simulated across synthetic model described in Table C.1. (a) $2 \mathrm{MHz}$, (b) $400 \mathrm{kHz}$ phase-shift and attenuation array resistivity measurements; (c) inverted layer-by-layer vertical, horizontal, and calculated true resistivities, (d) resistivity confidence intervals, and (e) layer-by-layer resistivity anisotropy coefficient. 


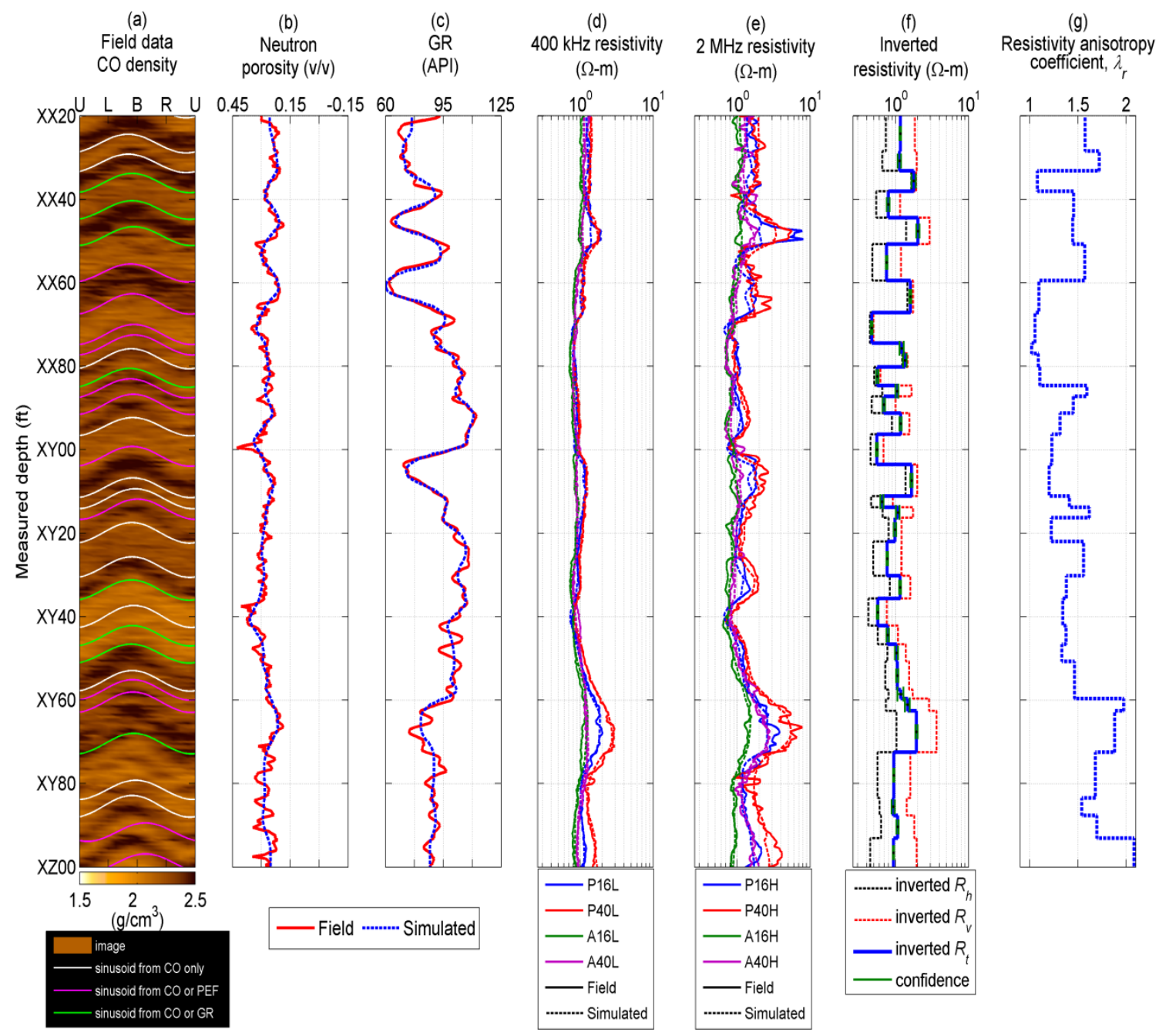

Figure C.2: Estimation of formation anisotropy across HA interval in field case example. (a) Field data compensated density image showing consolidated bed boundaries. Field and numerically simulated (b) neutron porosity, and (c) GR measurements. Field and numerically simulated array, 16- and 40in., propagation resistivity measurements, showing (d) $400 \mathrm{kHz}$, and (e) 2 $\mathrm{MHz}$ frequency curves. (f) Inverted layer-by-layer vertical, horizontal resistivities; and calculated true resistivity. (g) Calculated layer-by-layer anisotropy coefficient. 


\section{List of Symbols}

\begin{tabular}{|c|c|}
\hline$\theta$ & Azimuthal angle $\left(^{\circ}\right)$ \\
\hline$\Sigma$ & Capture cross section (cu) \\
\hline$\rho$ & Density $\left(\mathrm{g} / \mathrm{cm}^{3}\right)$ \\
\hline$\Delta$ & Departure \\
\hline$\delta$ & Dirac's delta function \\
\hline$\phi$ & Polar angle $\left({ }^{\circ}\right)$ \\
\hline$\phi$ & Apparent porosity $(\mathrm{v} / \mathrm{v})$ \\
\hline$\psi$ & Scalar flux function at $\mathbf{r}$ (particles $/ \mathrm{cm}^{2}-\mathrm{sec}$ ) \\
\hline$\beta_{b}$ & Apparent azimuth $\left({ }^{\circ}\right)$ \\
\hline$\theta_{b}$ & Apparent dip $\left(^{\circ}\right)$ \\
\hline$\rho_{b}$ & Bulk density $\left(\mathrm{g} / \mathrm{cm}^{3}\right)$ \\
\hline$\phi_{D}$ & Density porosity (v/v) \\
\hline$\alpha_{j}$ & Vector of azimuthal bins $\left({ }^{\circ}\right)$ \\
\hline$\rho_{m}$ & Matrix density $\left(\mathrm{g} / \mathrm{cm}^{3}\right)$ \\
\hline$\phi_{N}$ & Neutron near-to-far ratio porosity $(\mathrm{v} / \mathrm{v})$ \\
\hline$\phi_{s h}$ & Shale porosity (v/v) \\
\hline$\sigma_{t}$ & True conductivity $(\mathrm{S} / \mathrm{m})$ \\
\hline$\phi_{T}$ & Total porosity $(\%)$ \\
\hline$a$ & Neutron parameter coefficient \\
\hline $\mathrm{a}$ & Winsauer factor in Archie's equation ( ) \\
\hline $\mathbf{A}_{b, j}$ & Bed boundary sinusoid $(\mathrm{ft})$ \\
\hline $\mathrm{AmBe}$ & Americium-beryllium \\
\hline AO10 & 10 -in. array induction one foot resistivity $(\Omega-\mathrm{m})$ \\
\hline AO30 & 30 -in. array induction one foot resistivity $(\Omega-\mathrm{m})$ \\
\hline AO90 & 90 -in. array induction one foot resistivity $(\Omega-\mathrm{m})$ \\
\hline$A R$ & Axial resolution (in.) \\
\hline ARC & Schlumberger Array Compensated Resistivity \\
\hline
\end{tabular}


AT10 10-in. array induction two foot resistivity $(\Omega-\mathrm{m})$

AT30 30-in. array induction two foot resistivity $(\Omega-\mathrm{m})$

AT90 90-in. array induction two foot resistivity $(\Omega-\mathrm{m})$

$b \quad$ Density parameter coefficient $\left(\mathrm{cm}^{2} / \mathrm{g}\right)$

$B_{b} \quad$ Physical bed boundary ( $\mathrm{ft}$ )

BPHI Ratio-method, compensated neutron porosity in limestone units $(\mathrm{v} / \mathrm{v})$

C Minimization cost function

C Residual cost function

CNT Schlumberger compensated neutron tool

cps Counts per second

$C_{s h} \quad$ Volumetric concentration of shale $(\mathrm{v} / \mathrm{v})$

D $\quad$ Borehole diameter (ft)

d Measurement data vector

DFD Diffusion flux-difference

DPHI Density porosity in limestone matrix (v/v)

e Data misfit vector

E $\quad$ Energy of scattered particles (eV)

$E^{\prime} \quad$ Energy of incoming particles $(\mathrm{eV})$

ECS Elemental capture spectroscopy

EEC Environmental effects characterization

EPL Effective radial penetration length ( $\mathrm{ft}$ )

EVOI Effective volume of investigation $\left(\mathrm{ft}^{3}\right)$

fcps Neutron far detector count rates (cps)

FFM Fast-forward model

$F_{p} \quad$ Formation parameter $\left(\mathrm{cm}^{-1}\right)$

FSF 3D Flux sensitivity function

GCV Generalized cross-validation

$g_{d} \quad$ Neutron porosity calibration function, in dolomite units

$g_{l} \quad$ Neutron porosity calibration function, in limestone units

GR Gamma ray (API) 


\begin{tabular}{|c|c|}
\hline$g_{s}$ & Neutron porosity calibration function, in sandstone units \\
\hline$G_{\alpha}$ & Green's function kernel \\
\hline $\mathrm{HA} / \mathrm{HZ}$ & High-angle/horizontal \\
\hline HI & Hydrogen index \\
\hline$H I_{m}$ & Hydrogen index of the matrix ( ) \\
\hline$H P V$ & Hydrocarbon pore volume $(\%)$ \\
\hline $\mathbf{I}$ & Identity matrix \\
\hline ILD & Deep induction resistivity $(\Omega-\mathrm{m})$ \\
\hline ILM & Medium induction resistivity $(\Omega-\mathrm{m})$ \\
\hline$J$ & Total number of azimuthal sector-based bins \\
\hline $\mathbf{J}$ & Jacobian matrix \\
\hline$J_{0}$ & Zero-order Bessel function of the first kind \\
\hline$J_{1}$ & First-order Bessel function of the first kind \\
\hline$J_{\alpha}$ & Derivative of the Green's function kernel \\
\hline$k$ & Iteration count \\
\hline$L_{1 E}^{\prime}$ & Modified travel length $(\mathrm{cm})$ \\
\hline$L_{d}$ & Neutron diffusion length $(\mathrm{cm})$ \\
\hline LIR & Linear iterative refinement \\
\hline$L_{m}$ & Neutron migration length $(\mathrm{cm})$ \\
\hline$L_{s}$ & Neutron slowing-down length (cm) \\
\hline LWD & Logging-while-drilling \\
\hline$M$ & Total number of measured depth points \\
\hline$m$ & Archie's porosity exponent \\
\hline $\mathrm{MC}$ & Monte Carlo \\
\hline MCNP & Monte Carlo N-Particle software \\
\hline $\mathbf{m}_{n}$ & vector of polynomial coefficients \\
\hline$N$ & Total number of compositions \\
\hline$n$ & Archie's saturation exponent \\
\hline$N$ & Detector response (particles $/ \mathrm{cm}^{2}-\mathrm{sec}$ ) \\
\hline ncps & Neutron near detector count rates (cps) \\
\hline
\end{tabular}




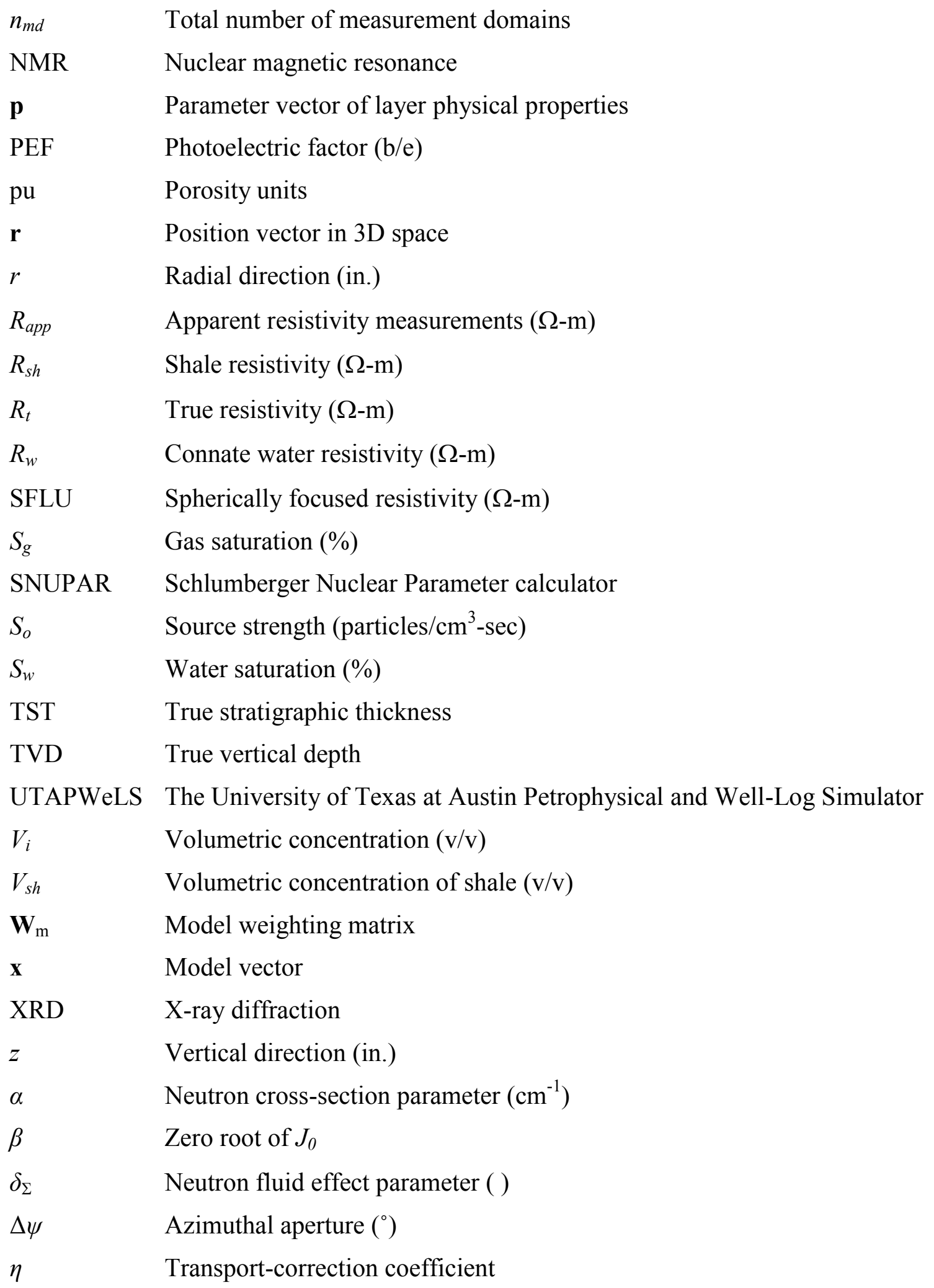




$\begin{array}{ll}\lambda & \text { Stabilization parameter } \\ \lambda_{r} & \text { Anisotropy coefficient } \\ \rho_{b} & \text { Volumetric bulk density }\left(\mathrm{g} / \mathrm{cm}^{3}\right) \\ \Sigma & \text { Macroscopic energy cross-section }\left(\mathrm{cm}^{-1}\right) \\ \phi_{N} & \text { Neutron near-to-far ratio porosity }(\mathrm{pu}) \\ \Psi & \text { Angular flux at } \mathbf{r} \text { of energy } E \text { and direction }\left(\text { particles } / \mathrm{cm}^{2} \text {-steradian-eV) }\right. \\ \mathbf{\Omega} & \text { Unit direction of scattered particles }() \\ \mathbf{\Omega}^{\prime} & \text { Unit direction of incoming particles ( ) } \\ \rho & \text { Density }\left(\mathrm{g} / \mathrm{cm}^{3}\right)\end{array}$

\section{Subscripts}

a

b

B

$b$

$\mathrm{CO}$

corr

$c w$

D

$d d$

$e$

ex

f

$g$

h

$h c$

$i$

j

ker
Absorption

Bulk

Base-case

Boundary

Compensated

Corrected

Connate-water

Density

Dual-detector

Electron

Extrapolation

Fluid

Grain

Horizontal

Hydrocarbon

Component index

Sector-based bin

Kerogen 


$\begin{array}{ll}l & \text { Lamina } \\ L S & \text { Long-space } \\ m & \text { Measurement depth point } \\ m f & \text { Mud-filtrate } \\ N & \text { Neutron } \\ n & n \text {-th order eigen term } \\ n_{b} & \text { Total number of layers } \\ n s h & \text { Non-shale } \\ n_{s p} & \text { Total number of data points } \\ p & \text { Pressure } \\ R & \text { Observation point (detector) } \\ s & \text { Scattering } \\ S & \text { Source point } \\ s d & \text { Single-detector } \\ s h & \text { Shale } \\ s n u & \text { SNUPAR } \\ S S & \text { Short-space } \\ t & \text { Transport } \\ v & \text { Vertical } \\ w f & \text { Water-filled }\end{array}$

\section{Superscripts}

$\begin{array}{ll}+ & \text { Adjoint } \\ k & \text { Nonlinear iteration } \\ m & m \text {-th order Taylor's series term } \\ T & \text { Transpose }\end{array}$




\section{References}

Aster, R. C., B. Borchers, and C. H. Thurber, 2005, Parameter estimation and inverse problems: Elsevier.

Booth, T. E., and J. S. Hendricks, 1984, Importance estimation in forward Monte Carlo calculations: Nuclear Technology and Fusion, 5, 90-100.

Bootle, R., M. Waugh, M. Bittar, F. Hveding, W. Hendricks, and S. Pancham, 2009, Laminated sand-shale formation evaluation using azimuthal LWD resistivity: Society of Petroleum Engineers Annual Technical Conference and Exhibition Proceedings, SPE 123890.

Butler, J., P. B. Kemshell, H. F. Locke, and M. J. Armishaw, 1992, $\mathrm{PC}^{2}-$ A porosity code for personal computers: Transactions of the Society of Petrophysicists and Well Log Analysts, 33rd Annual Logging Symposium, paper Q.

Case, C., C. C. Watson, and R. Lawrence, 1994, Sensitivity function technique for modeling nuclear tools: United Sates Patent 5,334,833.

Chemali, R., S. Gianzero, and S. M. Su, 1987, The effect of shale anisotropy on focused resistivity devices: Transactions of the Society of Petrophysicists and Well Log Analysts, 28th Annual Logging Symposium, paper H.

Clavier, C., W. Hoyle, and D. Meunier, 1971, Quantitative interpretation of thermal neutron decay time logs: Part I - Fundamentals and techniques: Journal of Petroleum Technology, 23, no. 6, 743-763.

Couët, B., and C. C. Watson, 1993, Monte Carlo differential neutron sensitivity calculations for nuclear well-logging: IEEE Transactions on Nuclear Science, 40, 928-932.

Dworak, D., J. Łoskiewicz, and M. Janik, 2001, Asymptotic solutions of neutron transport equation and the limits of correct use of diffusion approximation for rocks: Applied Radiation and Isotopes, 54, 845-848.

Ellis, D. V., C. R. Case, and J. M. Chiaramonte, 2003, Porosity from neutron logs I: Measurement: Petrophysics, 44, no. 6, 383-395.

Ellis, D. V., C. R. Case, and J. M. Chiaramonte, 2004, Porosity from neutron logs II: Interpretation: Petrophysics, 45, no. 1, 73-86.

Ellis, D. V., and J. M. Singer, 2007, Well logging for earth scientists: Springer.

Fertl, W. H., and D. J. Timko, 1971, A distinction of oil and gas in clean and shaly sands as derived from well logs: The Log Analyst, 12, no. 2. 
Gaymard, R. and A. Poupon, 1968, Response of neutron and formation density logs in hydrocarbon bearing formations: The Log Analyst, 9, no. 5.

Greenspan, E., 1976, Developments in perturbation theory: Advances in Nuclear Science and Technology, 9, 181-268.

Griffiths, R., 2009, Well placement fundamentals: Schlumberger.

Guo, P., A. Mendoza, and H. Yin, 2008, Impact of density tool depth of investigation on dip determination in high angle and horizontal wells: Transactions of the Society of Petrophysicists and Well Log Analysts, 49th Annual Logging Symposium, paper CCCC.

Guo, P., and J. Wang, 2009, Fast neutron log modeling in high angle and horizontal wells: Transactions of the Society of Petrophysicists and Well Log Analysts, 50th Annual Logging Symposium, paper TT.

Guzmán-Garcia, A. G., 2002, Analysis of LWD and wireline logs in highly deviated boreholes penetrating deepwater turbidites - Anisotropy, polarization horns, and net-sand derivatives: Transactions of the Society of Petrophysicists and Well Log Analysts, 43rd Annual Logging Symposium, paper UU.

Habashy, T. M., R. W. Groom, and B. R. Spies, 1993, Beyond the Born and Rytov approximations: A non-linear approach to electromagnetic scattering: Journal of Geophysical Research, 98, 1759-1775.

Hagiwara, T., 1997, Macroscopic anisotropy approach to analysis of thinly laminated sand/shale sequences - Sensitivity analysis of sand resistivity estimate and environmental corrections: Society of Petroleum Engineers Annual Technical Conference and Exhibition Proceedings, SPE 38669-MS.

Hansen, P. C., 1998, Rank-deficient and discrete ill-posed problems: numerical aspects of linear inversion: Society of Industrial and Applied Mathematics Monographs on Mathematical Modeling and Computation.

Heidari, Z., C. Torres-Verdín, A. Mendoza, O. Ijasan, and B. Voss, 2009, Rapid, interactive assessment of petrophysical and geometrical effects on density and neutron $\operatorname{logs}$ acquired in vertical and deviated wells: Society of Petroleum Engineers Annual Technical Conference and Exhibition Proceedings, SPE 124879 .

Heidari, Z., C. Torres-Verdín, and W. Preeg, 2012, Improved estimation of mineral and fluid volumetric concentrations in thinly bedded and invaded formations: Geophysics, 77, no. 3, WA79-WA98.

Heidari, Z., and C. Torres-Verdín, 2012, Estimation of dynamic petrophysical properties of water-bearing sands invaded with oil-base mud from the interpretation of multiple borehole geophysical measurements: Geophysics, 77, no. 6, D209-D227. 
Ijasan, O., C. Torres-Verdín, W. E. Preeg, and A. Mendoza, 2011, Rapid modeling of LWD nuclear measurements acquired in high-angle and horizontal wells for improved petrophysical and geometrical interpretation: Transactions of the Society of Petrophysicists and Well Log Analysts, 52nd Annual Logging Symposium, paper R.

Ijasan, O., C. Torres-Verdín, and W. E. Preeg, 2013, Fast modeling of borehole neutron porosity measurements with a new spatial transport-diffusion approximation: Geophysics, 78, no. 3, D151-D168.

Larionov, W. W., 1969, Borehole radiometry: Moscow, U.S.S.R., Nedra.

Lewins, J., 1965, Importance - The adjoint function: The physical basis of variational and perturbation theory in transport and diffusion problems: Pergamon Press Ltd.

Liu, Z., C. Torres-Verdín, G. L. Wang, A. Mendoza, and P. Zhu, 2007, Joint inversion of density and resistivity logs for the improved petrophysical assessment of thinlybedded clastic rock formations: Transactions of the Society of Petrophysicists and Well Log Analysts, 48th Annual Logging Symposium, paper VV.

Mallan, R. K., J. Ma, C. Torres-Verdín, and G. L. Wang, 2009, Joint radial inversion of resistivity and sonic logs to estimate in-situ petrophysical and elastic properties of formations: Society of Petroleum Engineers Annual Technical Conference and Exhibition Proceedings, SPE 124624.

Mandelis, A., 2001, Diffusion-wave fields: Mathematical methods and Green functions: Springer.

Mao, Z., 2001, The physical dependence and the correlation characteristics of density and neutron logs: Petrophysics, 42, no. 5, 438-443.

McKeon, D. C., and H. D. Scott, 1989, SNUPAR - A nuclear parameter code for nuclear geophysics applications: IEEE Transactions on Nuclear Science, 36, 1215-1219.

Mendoza, A., C. Torres-Verdín, and W. E. Preeg, 2007, Rapid simulation of borehole nuclear measurements based on spatial flux-scattering functions: Transactions of the Society of Petrophysicists and Well Log Analysts, 48th Annual Logging Symposium, paper O.

Mendoza, A., C. Torres-Verdín, and W. E. Preeg, 2010a, Linear iterative refinement method for the rapid simulation of borehole nuclear measurements: Part 1 Vertical wells: Geophysics, 75, no. 1, E9-E29.

Mendoza, A., C. Torres-Verdín, and W. E. Preeg, 2010b, Linear iterative refinement method for the rapid simulation of borehole nuclear measurements: Part 2 - Highangle and horizontal wells: Geophysics, 75, no. 2, E79-E90.

Mendoza, A., P. Gaillot, D. Mardon, J. Zhou, P. Guo, S. Wertanen, and H. Yin, 2010c, Quantitative formation evaluation in high-angle and horizontal wells using LWD measurements: Field applications of integrated log modeling workflow: Society 
of Petroleum Engineers Russian Oil and Gas Technical Conference and Exhibition Proceedings, SPE 135732.

Mendoza, A., O. Ijasan, C. Torres-Verdín, W. E. Preeg, J. Rasmus, R. J. Radtke, and E. Stockhausen, 2012, Inversion-based method for interpretation of logging-whiledrilling density measurements acquired in high-angle and horizontal wells: Geophysics, 77, no. 4, D113-D127.

Mimoun, J. G., C. Torres-Verdín, and W. E. Preeg, 2011, Quantitative interpretation of pulsed neutron capture logs: Part 1 - Fast numerical simulation: Geophysics, 76, no. 3, E81-E93.

Passey, Q. R., S. Creaney, J. B. Kulla, F. J. Moretti, and J. D. Stroud, 1990, A practical model for organic richness from porosity and resistivity logs: American Association of Petroleum Geologists Bulletin, 74, no. 12, 1777-1794.

Passey, Q. R., H. Yin, C. M. Rendeiro, and D. E. Fitz, 2005, Overview of high-angle and horizontal well formation evaluation - Issues, learnings, and future directions: Transactions of the Society of Petrophysicists and Well Log Analysts, 46th Annual Logging Symposium, paper A.

Plumb, R. A., and S. M. Luthi, 1989, Analysis of borehole images and their application to geologic modeling of an eolian reservoir: Society of Petroleum Engineers Formation Evaluation, 4, no. 4, 505-514.

Poupon, A., and J. Leveaux, 1971, Evaluation of water saturation in shaly formations: The Log Analyst, 12, no. 4, 3-8.

Quintero, L. F., and Z. Bassiouni, 1998, Porosity determination in gas-bearing formations: Society of Petroleum Engineers Permian Basin Oil and Gas Recovery Conference, SPE 39774.

Rendeiro, C., Q. R., Passey, and H. Yin, 2005, The conundrum of formation evaluation in high-angle and horizontal wells - Observations and recommendations: Society of Petroleum Engineers Annual Technical Conference and Exhibition Proceedings, SPE 96898.

Sanchez-Ramirez, J., C. Torres-Verdín, D. Wolf, G. L. Wang, A. Mendoza, Z. Liu, and G. Schell, 2010, Field examples of the combined petrophysical inversion of gamma-ray, density, and resistivity logs acquired in thinly-bedded clastic rock formations: Petrophysics, 51, no. 4, 247-263.

Schlumberger Limited, 2009, Log Interpretation Charts: Schlumberger.

Shetty, S., D. Omeragic, T. Habashy, J. Miles, J. Rasmus, R. Griffiths, and C. Morriss, 2012, Inversion-based workflows for interpretation of nuclear density images in high-angle and horizontal wells: Transactions of the Society of Petrophysicists and Well Log Analysts, 53rd Annual Logging Symposium, paper EEE. 
Spears, R. W., 2006, Lithofacies-based corrections to density-neutron porosity in a highporosity gas- and oil-bearing turbidite sandstone reservoir, Erha field, OPL 209, Deepwater Nigeria: Petrophysics, 47, no. 4, 294-305.

Stacey, W. M., 2007, Nuclear reactor physics: Wiley-VCH.

Stieber, S. J., 1970, Pulse neutron capture log evaluation - Louisiana Gulf Coast: Fall Meeting of the Society of Petroleum Engineers of American Institute of Mining, Metallurgical and Petroleum Engineers, SPE 2961.

Tittle, C. W., 1961, Theory of neutron logging I: Geophysics, 26, 27-39.

Uzoh, E. A., A. Mendoza, C. Torres-Verdín, W. E. Preeg, E. J. Stockhausen, and J. C. Rasmus, 2009, Influence of relative dip angle and bed thickness on LWD density images acquired in high-angle and horizontal wells: Petrophysics, 50, no. 3, 269293.

Voss, B., C. Torres-Verdín, A. Gandhi, G. Alabi, and M. Lemkecher, 2009, Common stratigraphic framework to simulate well $\operatorname{logs}$ and to cross-validate static and dynamic petrophysical interpretation: Transactions of the Society of Petrophysicists and Well Log Analysts, 50th Annual Logging Symposium, 200939813.

Watson, C. C., 1984, Monte Carlo computation of differential sensitivity functions: Transactions of the American Nuclear Society, 46, 655-656.

Watson, C. C., 1992, A spatial sensitivity analysis technique for neutron and gamma-ray measurements: Transactions of the American Nuclear Society, 65, 3-4.

X-5 Monte Carlo Team, 2003, MCNP - A general Monte Carlo N-particle transport code, version 5, developer's guide: University of California, Los Alamos National Laboratory, Los Alamos, New Mexico.

Xu, C., Z. Heidari, and C. Torres-Verdín, 2012, Rock classification in carbonate reservoirs based on static and dynamic petrophysical properties estimated from conventional well logs: Society of Petroleum Engineers Annual Technical Conference and Exhibition, SPE 159991.

Yin, H., X. Han, L. Xu, W. Guo, A. Shehata, and R. P. Gardner, 2006, Field and benchmark studies of LWD nuclear tool response in high angle and horizontal wells: Transactions of the Society of Petrophysicists and Well Log Analysts, 47th Annual Logging Symposium, paper AAA.

Yin, H., P. Guo, A. Mendoza, 2008, Comparison of processing methods to obtain accurate bulk density compensation and azimuthal density images from dualdetector gamma density measurements in high-angle and horizontal wells: Transactions of the Society of Petrophysicists and Well Log Analysts, 49th Annual Logging Symposium, paper M. 
Zhou, J., P. Guo, D. Mardon, Q. Passey, A. Mendoza, P. Gaillot, S. Wertanen, H. Yin, D. Fitz, and W. Nicosia, 2012, A high angle and horizontal well interpretation toolkit for quantitative formation evaluation and reservoir characterization: Society of Petroleum Engineers Kuwait International Conference and Exhibition Proceedings, SPE 163340.

Zhou, T., J. R. Miles, C. R. Case, J. M. Chiaramonte, and D. V. Ellis, 2009, A secondorder fast-forward model for gamma-gamma density tool: Society of Petroleum Engineers Annual Technical Conference and Exhibition Proceedings, SPE 124193. 


\section{Vita}

Olabode Ijasan received a B.Sc. (2006) in electrical and electronics engineering from the University of Lagos, Nigeria, and an M.Sc. (2010) in petroleum engineering from the University of Texas at Austin. In the summers of 2011 and 2012, he worked as a research intern at Schlumberger-Doll Research, Cambridge, Massachusetts, developing fast and accurate methods for simulating borehole nuclear measurements. He also worked at ExxonMobil Upstream Research and Exploration Companies in the summers of 2009 and 2010, respectively. Upon completion of his doctoral degree, Olabode will join ExxonMobil to work in formation evaluation and the geosciences. He is a member of SEG, SPE, SPWLA, and IEEE.

Permanent e-mail address: oijasan@utexas.edu This dissertation was typed by Olabode Ijasan. 